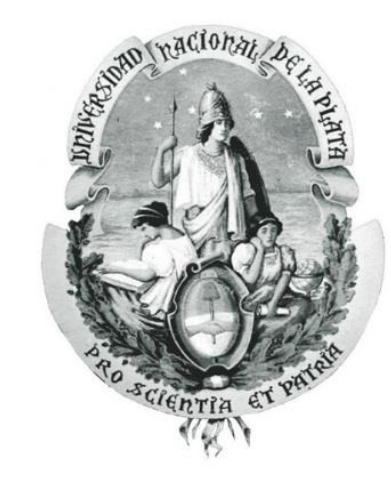

\title{
Procesamiento de señales aplicado a dispositivos de ayuda para personas con discapacidades motoras
}

Tesis de doctorado

\author{
MARCELO ALEJANDRO HABERMAN
}

\author{
Presentada ante la Facultad de Ingeniería de la \\ Universidad Nacional de La Plata \\ como requisito para la obtención del grado académico de \\ DOCTOR EN INGENIERIA
}

Dirección de Tesis:

Director: Dr. Enrique Mario Spinelli

Codirectora: Ing. Graciela Mabel Toccaceli

Jurado de Tesis:

Dra. Lucía Isabel Passoni

Dr. Pedro David Arini

Fecha de defensa oral y pública: 15 de Abril de 2016 



\section{Resumen}

Las afecciones motrices más graves, que involucran la parálisis o pérdida de los miembros, pueden imposibilitar a quien las padece para la realización de tareas de índole doméstico, necesitando ayuda externa para concretarlas. El avance tecnológico ha abierto una carrera incesante en el desarrollo de dispositivos que permiten aumentar el grado de autonomía del discapacitado, aún en personas con afecciones neurológicas y motrices severas, a las que se les suma la pérdida de la capacidad del habla.

En la presente tesis se estudian los dispositivos de ayuda que son controlados por los usuarios a partir de señales biopotenciales que los mismos pueden modular a voluntad. La medición de biopotenciales requiere de técnicas analógicas para reducir el impacto del ruido y la interferencia electromagnética en la calidad de las señales, dado los bajos niveles de tensión y las altas impedancias que presentan los biopotenciales. Una vez medidas las señales de interés se aplican técnicas digitales para detectar las órdenes voluntarias del usuario codificadas de distintas maneras en dichas señales biológicas.

En la primera parte de esta tesis se analizan problemáticas de la instrumentación de biopotenciales aplicada a dispositivos de ayuda, como las condiciones de acoplamiento de interferencia en entornos domésticos y distintas técnicas para la reducción de la tensión de modo común. También se evalúa la posibilidad de adquirir los biopotenciales en forma aislada mediante electrodos capacitivos.

En la segunda parte de la tesis se plantea como caso particular de aplicación, un dispositivo de ayuda de tipo switch controlado por señales de EMG. Se realizó un análisis de distintas técnicas de procesamiento de la señal de EMG para la detección de contracciones y se proponen nuevos métodos de procesamiento que mejoran la robustez de la detección ante cambios en los niveles de ruido y de señal.

Finalmente los temas tratados a lo largo de la tesis se integran en la implementación de un prototipo de dispositivo de ayuda de tipo switch controlado por señales de EMG, robusto ante distintas condiciones de uso y que prácticamente no requiere calibración ni ajustes para operar sobre distintos músculos y usuarios. 
Resumen 


\section{Nomenclatura}

$A B$ :

ADC:

$\mathrm{BCl}:$

$\mathrm{Cl}$

CMRR:

DA:

$D A B:$

EEG:

ELA:

EMG:

PC:

SE:

SPI:

Sps:
Amplificador de Biopotenciales

Conversor Analógico-Digital (Analog-to-Digital Converter)

Interfaz Cerebro-Computadora (Brain Computer Interface)

Circuito Integrado

Rechazo de modo común (Common Mode Reject Rate)

Dispositivo de Ayuda

Dispositivo de Ayuda controlado por Biopotenciales

Electro-Encéfalo-Grama

Esclerosis Lateral Amiotrófica

Electro-Mío-Grama

Computadora Personal (Personal Computer)

Salida simple (Single Ended)

Serial Peripheral Interface

Muestras por segundo (Samples per second) 
Nomenclatura 


\section{Índice}

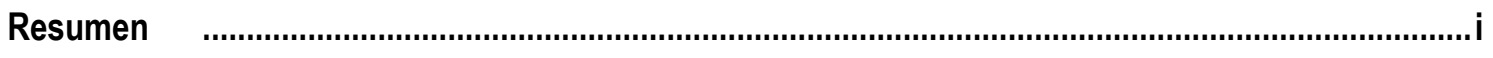

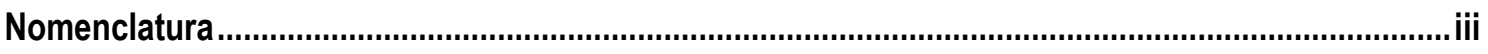

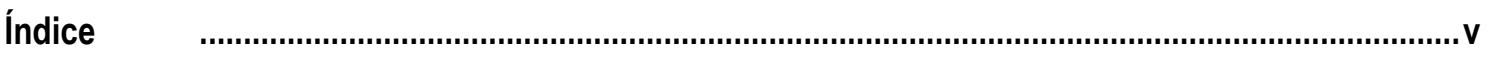

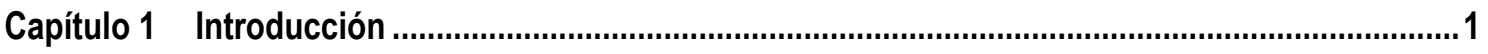

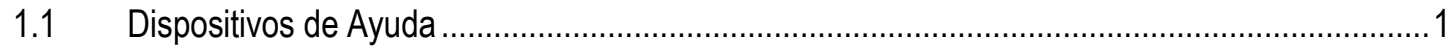

1.1.1 Dispositivos de Ayuda controlados por señales biopotenciales .........................................

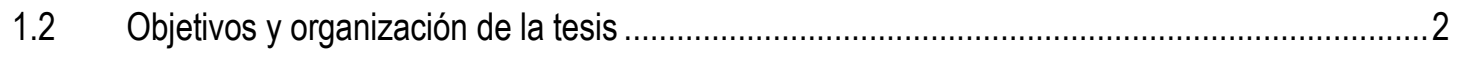

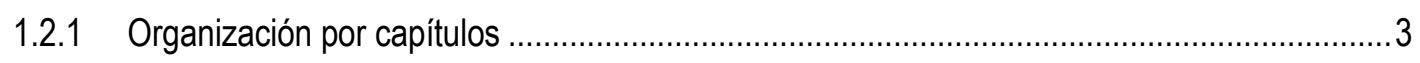

Capítulo 2 Biopotenciales e Interferencia Electromagnética .....................................................5

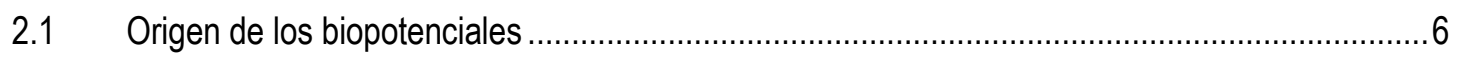

2.2 Señales biomédicas ....................................................................................................

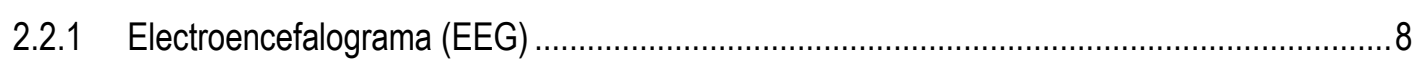

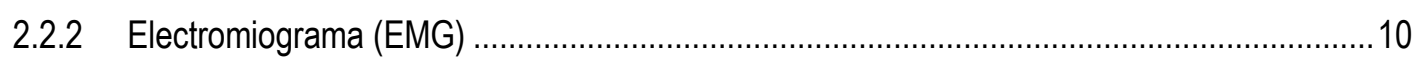

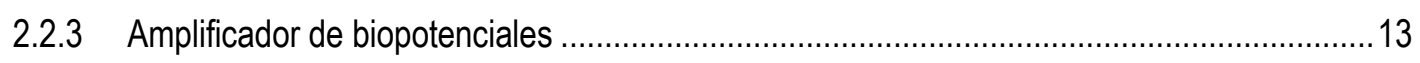

2.3 Interferencia Electromagnética en la adquisición de biopotenciales ........................................14

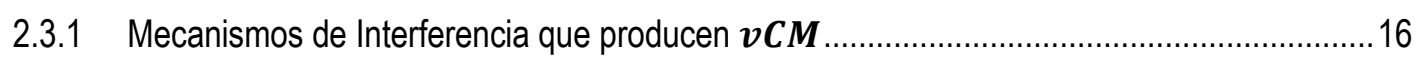

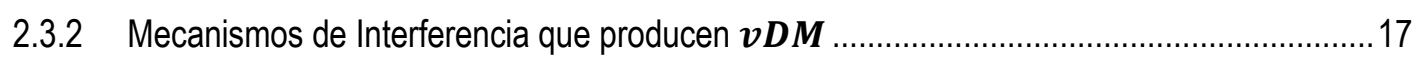

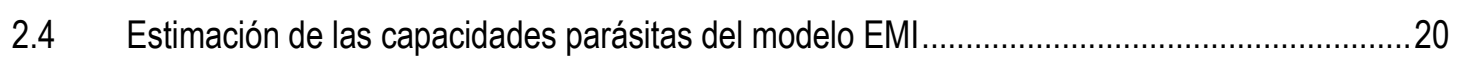

2.4.1 Método utilizado para estimación de capacidades parásitas ..............................................20

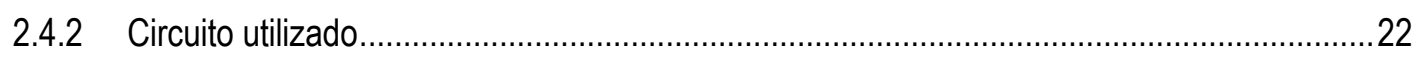

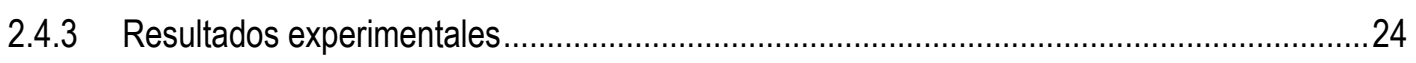

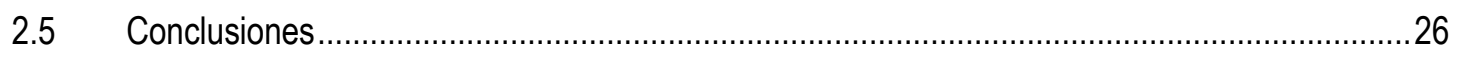

Capítulo 3 Electrodos para Dispositivos de Ayuda.................................................................29

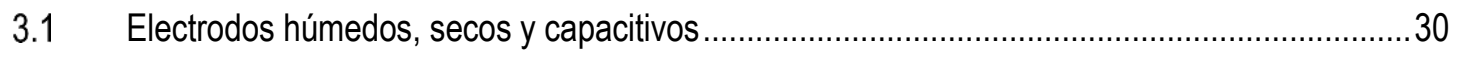

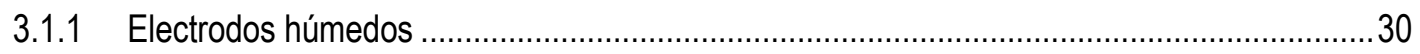

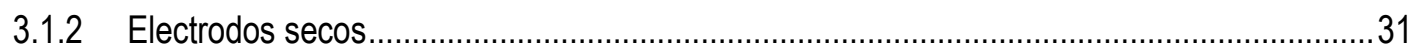

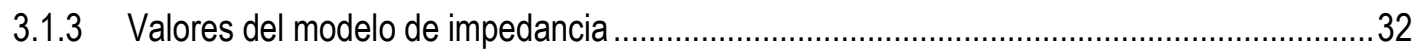

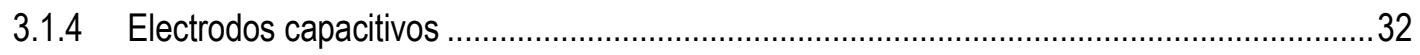

3.2 Electrodos activos desarrollados para un DA controlado por EMG ..........................................3

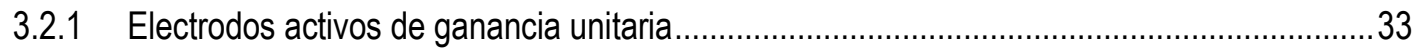

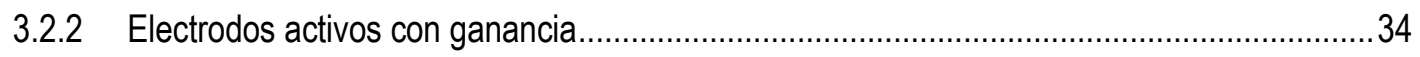

3.2.3 Electrodos activos con ganancia y realimentación de modo común...................................35 


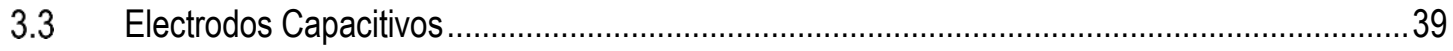

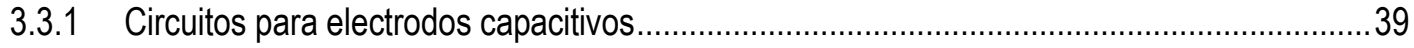

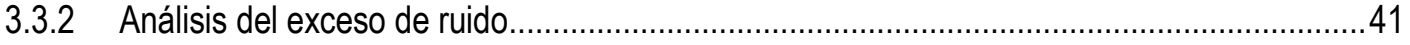

3.4 Electrodo capacitivo desarrollado para EMG ……............................................................ 44

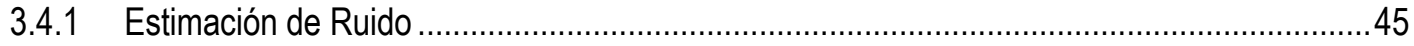

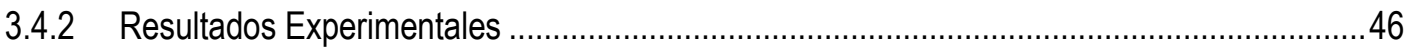

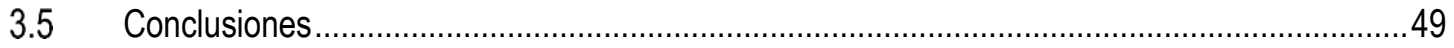

Capítulo 4 Reducción de interferencia de modo común ............................................................51

4.1 Reducción de $\boldsymbol{v} \mathbf{C M}$ por realimentación negativa ...........................................................52

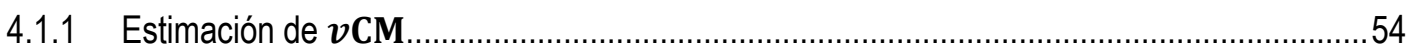

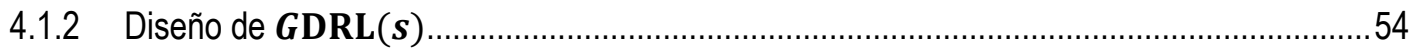

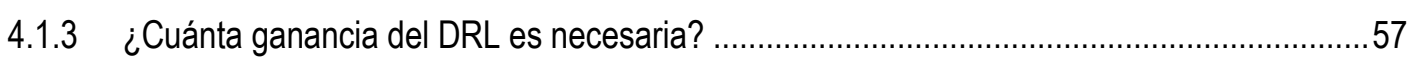

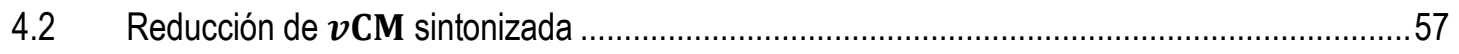

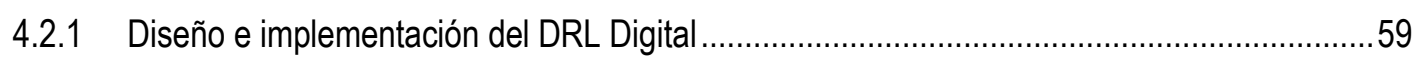

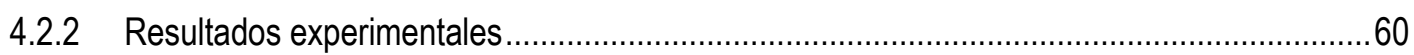

4.2.3 Esquema de adquisición multicanal basado en amplificadores Single-Ended y el DDRL ....60

$4.3 \quad$ Reducción de $\boldsymbol{v} \mathbf{C M}$ en sistemas capacitivos......................................................................62

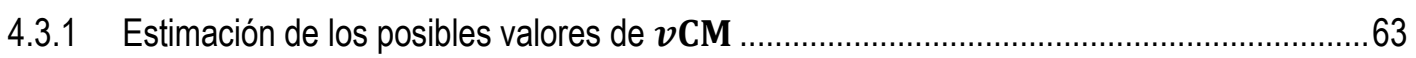

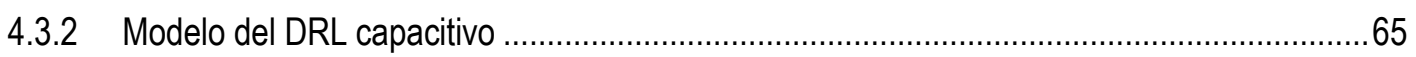

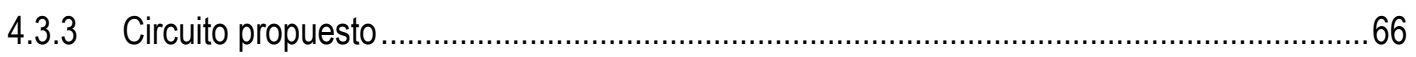

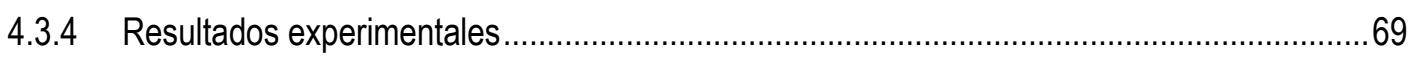

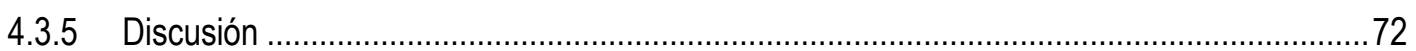

4.4 Sensor activo de EMG con reducción de modo común in-situ ................................................72

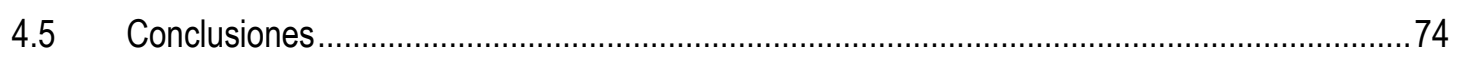

Capítulo 5 Procesamiento digital de EMG para Dispositivos de Ayuda .......................................77

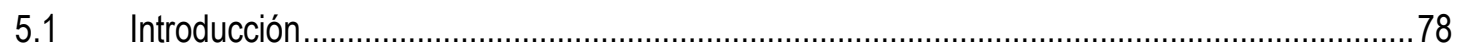

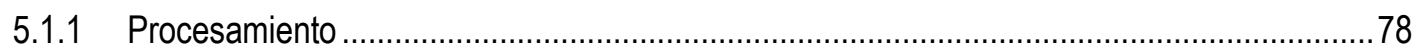

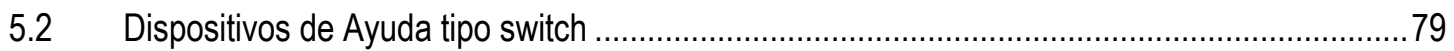

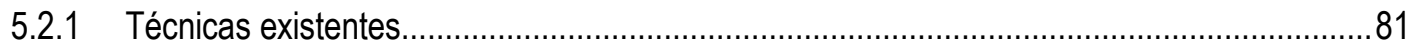

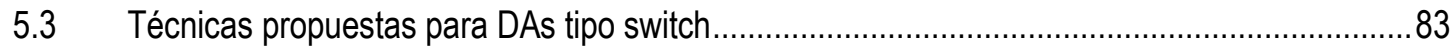

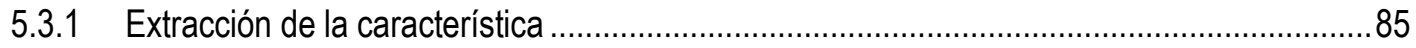

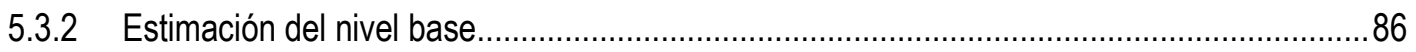

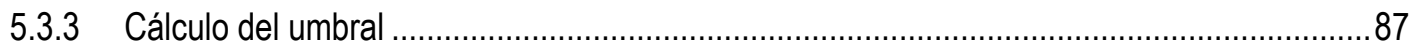

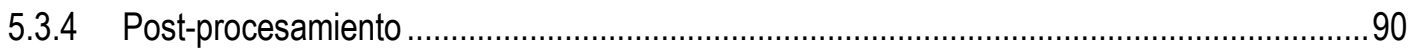

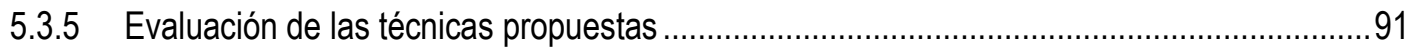

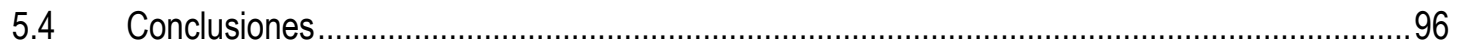


Capítulo 6 Implementación de un DA tipo switch

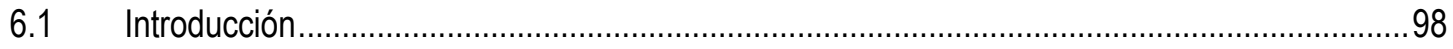

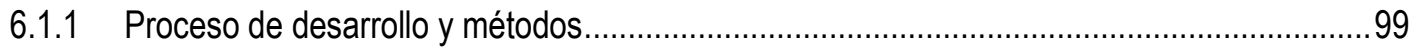

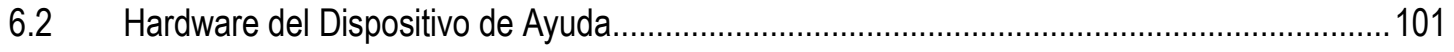

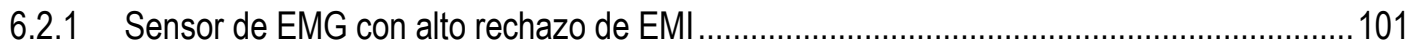

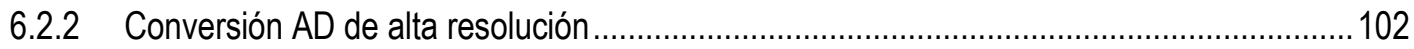

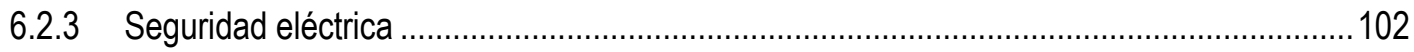

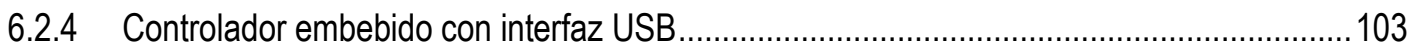

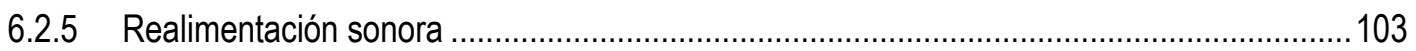

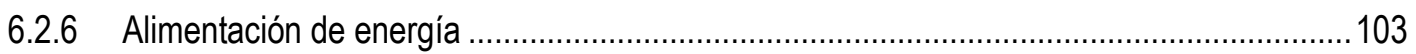

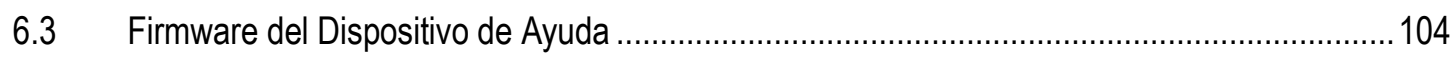

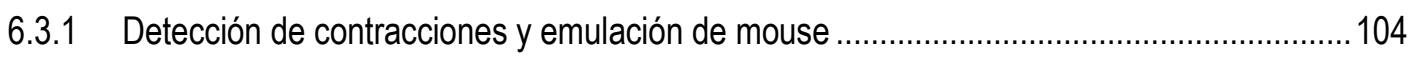

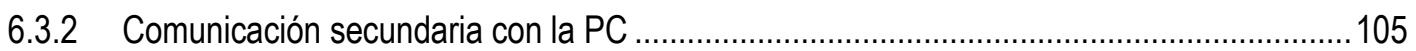

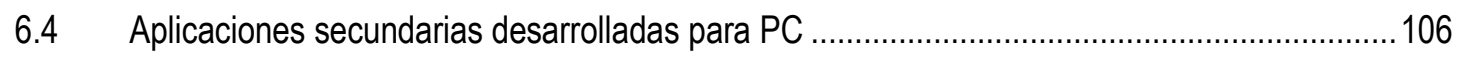

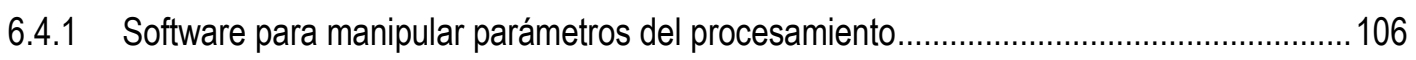

6.4.2 Aplicaciones para visualización y registro de señales internas del procesamiento .............107

6.4.3 Bloque para Simulink de Matlab ......................................................................................... 108

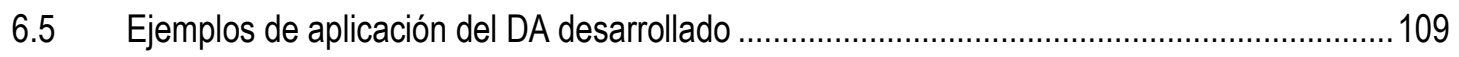

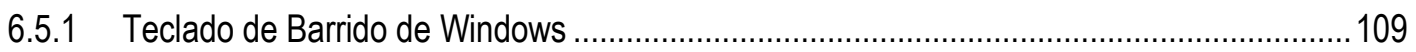

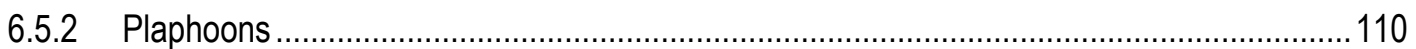

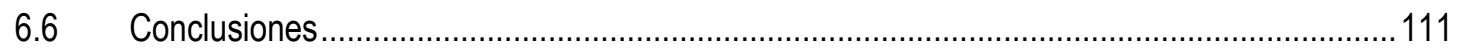

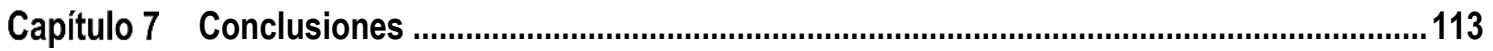

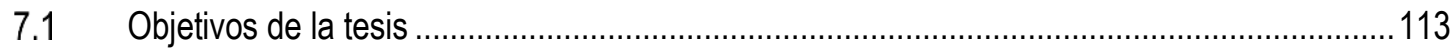

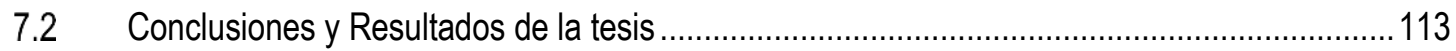

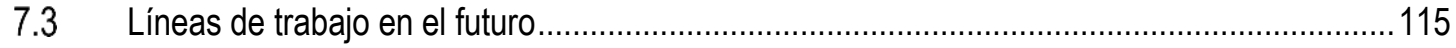

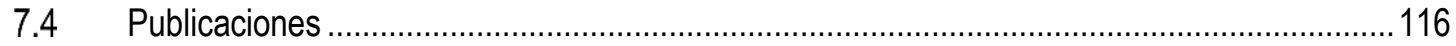

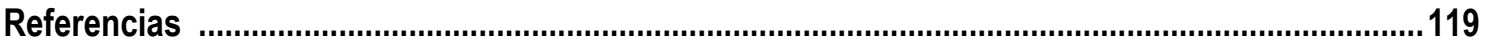


Índice 


\section{Capítulo 1}

\section{Introducción}

\subsection{Dispositivos de Ayuda}

Las discapacidades motoras son disfunciones en el aparato locomotor, que pueden producir limitaciones posturales, de desplazamiento o de coordinación del movimiento.

Son diversos los problemas asociados a la discapacidad motriz; entre ellos pueden mencionarse: movimientos incontrolados, dificultades de coordinación, alcance limitado, fuerza reducida, habla ininteligible, dificultad con la motricidad fina y gruesa, mala accesibilidad al medio físico. Las afecciones más graves, que involucran la parálisis o pérdida de los miembros, pueden imposibilitar a quien las padece para la realización de tareas de índole doméstico, necesitando ayuda externa para concretarlas.

El avance tecnológico ha abierto una carrera incesante en el desarrollo de dispositivos que permiten aumentar el grado de autonomía del discapacitado, aún en personas con afecciones motrices severas, a las que se les suma la pérdida de la capacidad del habla.

Estos dispositivos no pretenden la recuperación total de las capacidades motrices afectadas, sino dotar a la persona de algún grado de libertad para la concreción de determinadas tareas o la compensación de determinadas funciones (Henderson et al. 2008). Aun las más simples, como encender/apagar una luz, mover una silla de ruedas o cambiar el canal del televisor, pueden mejorar sustancialmente su independencia y con esto su calidad de vida.

Dada la falta de un término unificado que englobe estos dispositivos tan diversos (pueden ser aplicaciones de software, prótesis puramente mecánicas, prótesis mecánicas con sensores implantados, etc.) es que se decide utilizar el término "Dispositivos de Ayuda" (DA). Esta denominación se refiere, en general, a aquellos sistemas que apuntan a reemplazar parcialmente la funcionalidad motriz ausente. Por ejemplo, el manejo de una Computadora Personal (PC: Personal Computer) mediante comandos de voz, por parte de alguien con disfunciones en los miembros superiores, constituye un DA que reemplaza el movimiento de los miembros afectados únicamente en la tarea de utilizar una PC. Precisamente, los DA están orientados a efectuar tareas puntuales, generalmente del orden de lo cotidiano, que no pueden realizarse por las "vías convencionales" debido 
a una discapacidad motriz. De este modo, a través de canales alternativos, un DA permite ejecutar la "voluntad" del usuario.

Cuando la persona no tiene afectadas sus capacidades motoras, esta "voluntad" se materializa en impulsos eléctricos, que tienen un camino directo (Deutsch y Deutsch 1993):

$$
\text { Voluntad de movimiento } \rightarrow \text { Cerebro } \rightarrow \text { Nervio } \rightarrow \text { Músculo } \rightarrow \text { Ejecución del movimiento }
$$

Dependiendo del tipo de afección, este trayecto, o parte del mismo se interrumpe. Normalmente el camino interrumpido es reemplazado por otro "alternativo" provisto por un DA que se establece a través de miembros que conserven algún grado funcionalidad motora y/o nerviosa. Un ejemplo paradigmático es el manejo de una silla de ruedas: un DA que reemplaza la función de las piernas para el traslado. El usuario la comanda con sus brazos, constituyendo estos el canal de comunicación alternativo para la voluntad de desplazamiento de la persona.

\subsubsection{Dispositivos de Ayuda controlados por señales biopotenciales}

La información transportada en los impulsos eléctricos desde el cerebro al músculo puede ser observada mediante la captura de señales electro-fisiológicas denominadas biopotenciales, pudiendo así establecerse canales alternativos para la comunicación de la voluntad del usuario que se manifiesta en estas señales.

Los Dispositivos de Ayuda controlados por Biopotenciales (DABs) son sistemas complejos compuestos en general por las siguientes etapas o módulos:

- Adquisición de las señales biopotenciales: Este bloque incluye el procesamiento de señal analógico que se realiza previo a la digitalización, cuyas partes principales son los electrodos y el amplificador de biopotenciales. Además, para mejorar la relación señal a ruido (SNR), se utilizan circuitos de realimentación de modo común y filtros.

- Procesamiento digital de las señales: La representación más abstracta de este bloque es la de una "caja negra" cuyas entradas son los biopotenciales digitalizados y sus salidas son señales de control para una aplicación específica (ejemplos de "aplicaciones" son el control de una prótesis, el encendido/apagado de una lámpara o la manipulación de un televisor). Es decir, este bloque puede pensarse como un traductor entre las señales biopotenciales y las señales de control o comando de una aplicación objetivo.

- Aplicación e Interfaz de realimentación al usuario: Representa la aplicación del DAB en el entorno del usuario, como los elementos actuadores que convierten las señales de control en acciones concretas (relés, motores, software) y las distintas maneras en que el usuario obtiene la realimentación de las acciones que realiza (pantallas, luces, audio).

\subsection{Objetivos y organización de la tesis}

Un objetivo de la presente tesis es lograr la articulación de los diferentes campos de conocimiento que intervienen en el diseño, implementación y uso de los DABs por parte de sus usuarios.

A lo largo de la tesis se tratan las problemáticas asociadas a la medición de señales biopotenciales, su procesamiento analógico y digital, así como su integración en un mismo sistema. Esto debe hacerse teniendo en cuenta que el objeto de estudio es un DAB cuya finalidad es brindar confort a sus usuarios, facilitándoles la comunicación y el acceso a su entorno. Esto impone fuertes restricciones al diseño de los bloques que componen 
el $\mathrm{DAB}$, por ejemplo, las etapas analógicas deberán operar durante largos períodos en condiciones de interferencia y perturbaciones eléctricas no controladas, y los algoritmos de procesamiento digital deberán ser robustos ante distintas variaciones de los parámetros de las señales.

Como se verá en el Capítulo 2, existen diferentes tipos de biopotenciales, que se distinguen principalmente por el órgano o tejido que los produce, obteniéndose señales de amplitudes, morfología y contenido espectral muy diverso. No todos los biopotenciales son aptos para extraer información de la voluntad de una persona 0 , dicho de otro modo, una persona puede modular o modificar voluntariamente las características de sólo algunas señales biopotenciales. Los más susceptibles de ser modulados voluntariamente son las señales tomadas directamente sobre el camino que va del cerebro al músculo (ver Figura 1-1).

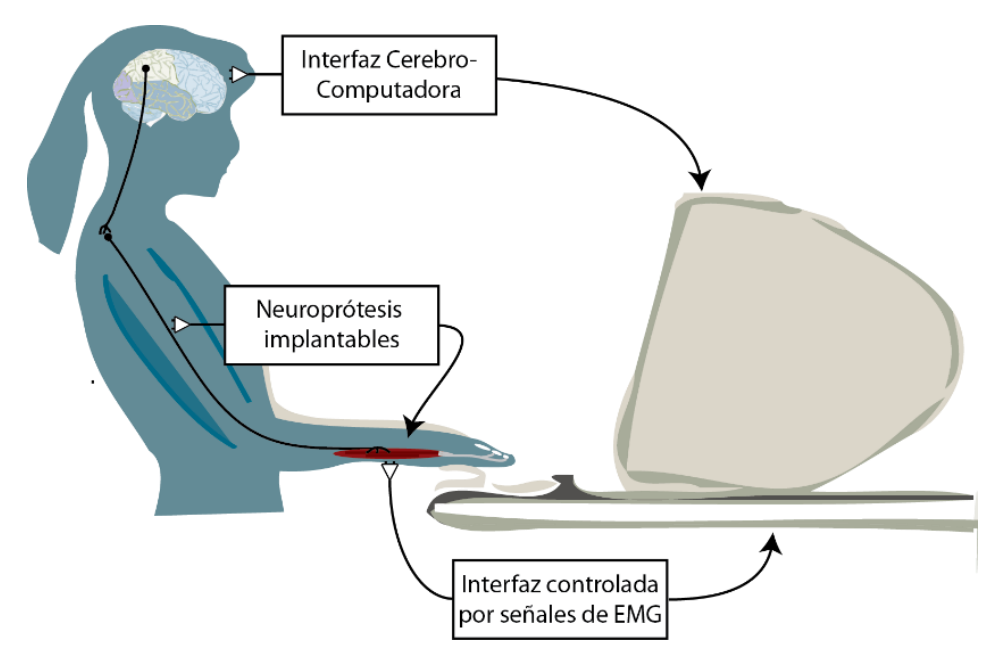

Figura 1-1: Ejemplo del distintos tipos de DAB que toman la señal biopotencial en distintos lugares del recorrido cerebro-nervio-músculo que realizan las señales eléctricas del sistema nervioso.

La tesis se organiza en dos ejes principales. El primero comprende temas de instrumentación y procesamiento analógico de biopotenciales, entre el Capítulo 2 y el Capítulo 4, donde se tratan temas generales de la instrumentación de biopotenciales, se presentan problemas particulares de su aplicación en DABs y se aportan soluciones originales. El segundo eje, que se desarrolla a partir del Capítulo 5, aborda el procesamiento digital de las señales de EMG y el desarrollo de un prototipo funcional de DA tipo switch controlado por señales de EMG, que integra los distintos temas tratados a lo largo de la tesis, aplicándose varios de los resultados obtenidos.

La dinámica en el avance de la tesis, llevó a la focalización en DAs controlados por señales de EMG. Por lo tanto, si bien algunos temas de la primera parte se abordan de manera general, otros tanto son específicos para las señales de EMG.

\subsubsection{Organización por capítulos}

La medición de las señales biopotenciales puede realizarse mediante electrodos superficiales, que se colocan sobre la piel, o mediante electrodos implantados. Estos últimos permiten obtener fácilmente señales de muy buena calidad, pero implican riesgos de infecciones e incomodidad en los usuarios, por lo cual no son analizados en la tesis. Esta tesis trata únicamente de DAs, donde los biopotenciales se miden de forma no invasiva, es decir con 
el mínimo impacto posible en el confort del usuario. Desde el punto de vista de la ingeniería electrónica esto implica tratar con condiciones de medida propensas a interferencias y perturbaciones; por lo tanto en el Capítulo 2 se recopilan las diferentes maneras en que las señales que se desean medir pueden ser perturbadas, estimándose los valores que pueden tomar dichas perturbaciones, a partir de modelos eléctricos que representan los mecanismos de acoplamiento de interferencias. Así mismo, se aportan los resultados de medidas experimentales realizadas para caracterizar los parámetros de dichos modelos en situaciones de uso común para DAs.

En el Capítulo 3 se trata el tema de los electrodos superficiales y se presentan los dos tipos de electrodos más utilizados: húmedos y secos; según hagan uso o no de pastas o geles electrolíticos. Se analiza las ventajas y desventajas de su uso en DABs, se presentan diseños de electrodos implementados para un DAB controlado por señales de EMG y resultados experimentales. En el mismo capítulo se presentan los electrodos capacitivos: una tecnología emergente en la adquisición de biopotenciales, abordando el aspecto más crítico que tienen, su exceso de ruido. Se presentan resultados experimentales obtenidos con electrodos capacitivos desarrollados para adquirir señales de EMG, a fin de comparar su desempeño con el de los electrodos convencionales y determinar su viabilidad para el uso en DAs.

En el Capítulo 4 se trata la reducción de interferencia de modo común por realimentación negativa. Se proponen diferentes circuitos desarrollados que mejoran las características de reducción del modo común frente a las soluciones clásicas.

El Capítulo 5 presenta distintos algoritmos desarrollados particularmente para el control de una interfaz tipo ONOFF mediante señales de EMG. Dado que dichos algoritmos fueron diseñados para que los DAB sean prácticos, además de robustos, no requieren entrenamiento por parte del usuario y prácticamente no necesitan calibraciones o ajustes para su puesta en funcionamiento. Se presentan diferentes resultados experimentales que muestran el buen desempeño de dichas técnicas.

En el Capítulo 6 se presenta la implementación del DAB desarrollado durante el transcurso de la tesis, el cual integra los diversos temas tratados en los capítulos previos.

Finalmente, en el Capítulo 7 se presentan los resultados y conclusiones de la tesis, así como las líneas de trabajo abiertas. 


\section{Capítulo 2}

\section{Biopotenciales e Interferencia Electromagnética}

En el presente capítulo se describe someramente el origen de la actividad electrofisiológica, que da lugar a las señales biopotenciales utilizadas para el comando de Dispositivos de Ayuda y se describen características de interés más relevantes de las señales electro-encéfalo-gráficas y electro-mio-gráficas, utilizadas para el control de distintos DAs.

Se presenta el modelo de EMI (ElectoMagnetic Interference) que representa, con un circuito de parámetros concentrados, las distintas interacciones del circuito de medida de biopotenciales con los campos eléctricos y magnéticos producidos por fuentes externas de interferencia, especialmente por la línea de energía eléctrica y los aparatos eléctricos conectados a la misma. Este modelo describe los diferentes mecanismos de acoplamiento de interferencia y permite estimar posibles valores de amplitud de la misma, que van a sumarse a la señal que se desea medir. A partir del estudio de dichos mecanismos de acoplamiento es posible realizar acciones sobre el diseño del sistema de medida de forma tal de mitigar su efecto.

Los DAs basados en biopotenciales deben funcionar en ambientes no controlados, a diferencia de los laboratorios de investigación y los consultorios médicos. Las condiciones de EMI que se imponen en los ambientes domésticos pueden llegar a ser muy agresivas, dado que es imposible para quien diseña, o asiste en el uso de estos equipos, controlar la disposición de fuentes de EMl externas. Por estos motivos se realizaron mediciones de los parámetros más críticos del modelo EMI en distintas condiciones de acoplamiento de interferencia plausibles de ocurrir en ambientes domésticos. El presente capítulo concluye con los datos experimentales obtenidos. 


\subsection{Origen de los biopotenciales}

Las células excitables, como aquellas que componen el cerebro, nervios y músculos, tienen la capacidad de producir y propagar potenciales de acción. Estos potenciales, de origen iónico, se originan en la membrana celular, es decir se establecen entre el interior y el exterior de la célula. La forma típica de un potencial intracelular, registrado mediante un electrodo insertado en el interior de la célula respecto del potencial en el exterior se muestra en la Figura 1- (Moritani et al 2004, Clark 2009).

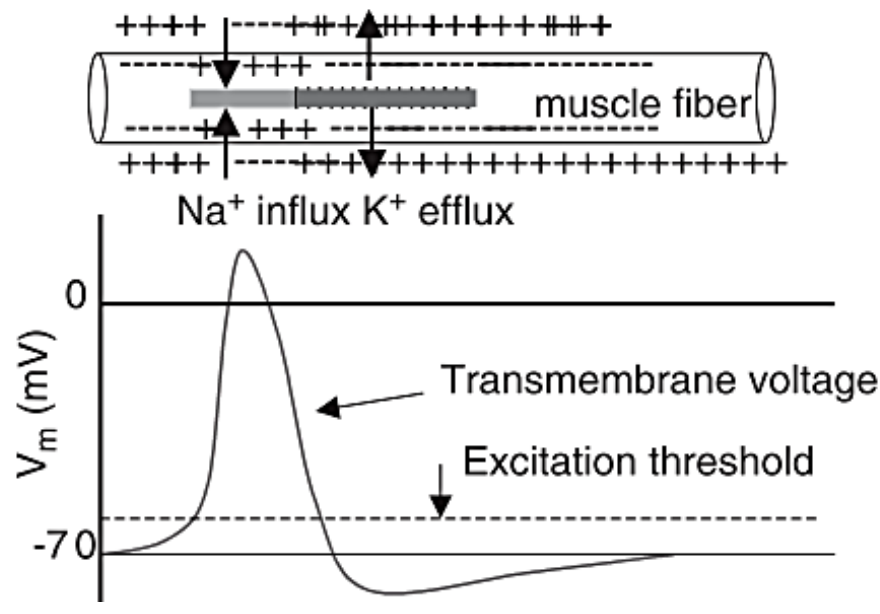

Figura 2-1: Forma característica del potencial de acción en una fibra muscular (Figura tomada de Moritani et al 2004).

Cuando se observan biopotenciales colocando electrodos sobre la piel, las señales observadas son muy distintas al potencial de acción de la figura previa. Por un lado, sobre la superficie del cuerpo no se registran potenciales intracelulares sino potenciales extracelulares, que son la manifestación de los primeros en el exterior al imprimir corrientes sobre los tejidos circundantes, que ofician de conductor de volumen (Clark 2009, Farina et al 2004). Por el otro, generalmente no se observan potenciales de acción individuales, sino la superposición de un gran número de éstos originados en distintas células, que se combinan en el mencionado conductor de volumen.

La amplitud y la morfología de las señales observadas dependen de múltiples factores, como el tipo de células involucradas, la cantidad de ellas y la distancia a la superficie, entre otras. Así, las señales electro-cardio-gráficas (ECG) que involucran una gran cantidad de células operando en forma sincronizada, tienen una forma bien definida y una amplitud de hasta algunos $\mathrm{mV}$. Un caso muy distinto son las señales electro-encefalo-gráficas (EEG), donde la sincronización es un estado patológico (epilepsia), normalmente tienen aspecto aleatorio y su amplitud es menor.

La técnica más extendida para adquirir biopotenciales es utilizando electrodos metálicos, que se colocan sobre la piel interponiendo un electrolito líquido, en pasta o en gel. Por esto se denominan electrodos húmedos.

Como se mencionó anteriormente, los biopotenciales son de naturaleza iónica. Los electrodos ofician de transductores, y los transforman en señales electrónicas, factibles de ser procesadas y registradas. Este complejo proceso de transducción de corrientes iónicas a corrientes electrónicas (Neuman 2009a), se produce en la interfaz electrodo-electrolito-piel. 


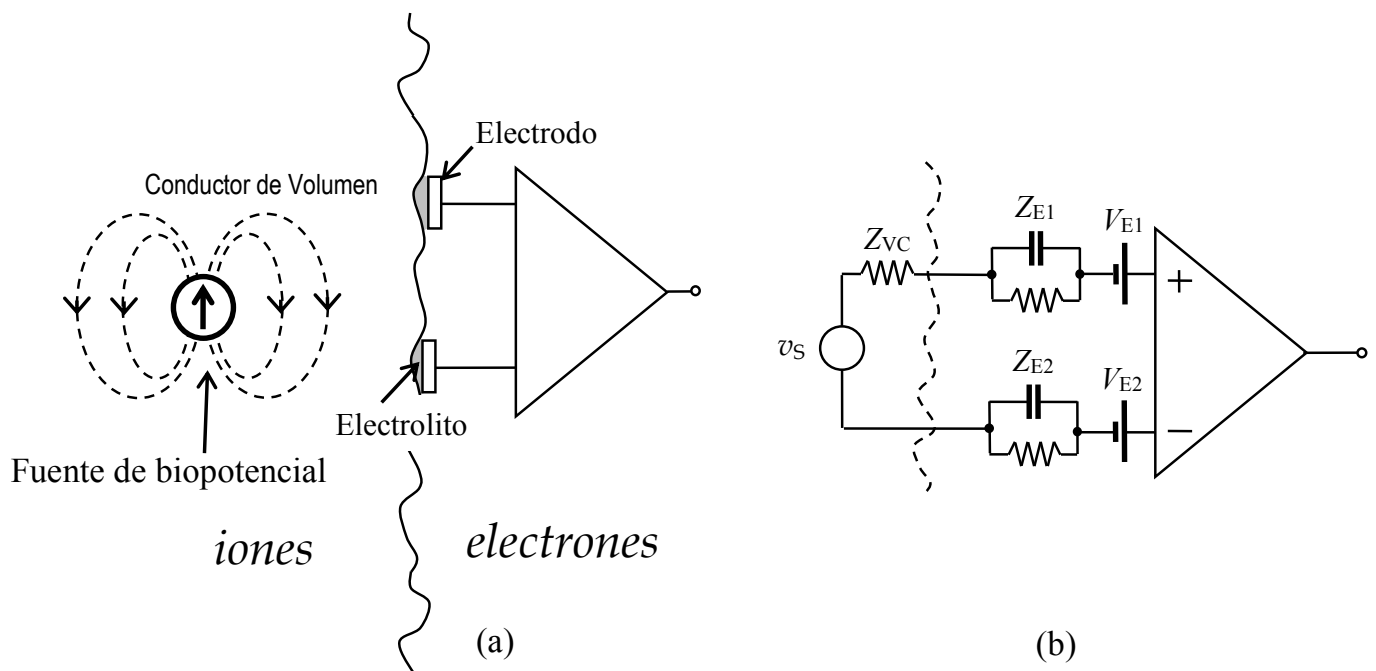

Figura 2-2: (a) Fuente de biopotencial modelada como dipolo de corriente en un conductor de volumen. (b) Modelo para la instrumentación del biopotencial como un generador de tensión $\boldsymbol{v}_{\mathbf{S}}$ y la impedancia del tejido $\boldsymbol{Z}_{\mathbf{V C}}$ que incluye la impedancia $\boldsymbol{Z}_{\mathbf{E i}}$ y un potencial continuo $\boldsymbol{V}_{\mathbf{E i}}$ de los electrodos.

En la Figura 2-2(a) puede verse la fuente de biopotencial modelada como un dipolo de corriente que generará el desplazamiento de portadores de carga (iones) en el conductor de volumen (tejido circundante) y en las cercanías de dicha fuente de biopotencial se observa un par de electrodos conectados a un Amplificador de Biopotenciales $(\mathrm{AB})$ que captarán una diferencia de potencial $v_{\mathrm{S}}$ desarrollada por las corrientes circulantes en el conductor de volumen $Z_{\mathrm{VC}}$.

Para los fines de diseño de un sistema de adquisición de biopotenciales, es suficiente con considerar que se accede al biopotencial a través de una impedancia de electrodo $Z_{\mathrm{Ei}}$ y de un potencial de continua $V_{\mathrm{Ei}}$ con origen en la semipila metal-electrolito (ver Figura 2-2(b)). El valor de $Z_{\mathrm{Ei}}$ depende de múltiples factores y puede ser de hasta cientos de $\mathrm{k} \Omega$ (Geddes y Valentinuzzi 1973) y es mucho mayor que el de $Z_{\mathrm{VC}}$. El potencial de continua, denominado tensión de offset de electrodo, puede tomar valores de hasta cientos de $\mathrm{mV}$, es decir varios órdenes de magnitud mayores que la amplitud de las señales de interés. Un modelo más completo de la impedancia de electrodo incluye un generador de ruido, cuyo valor excede al esperable por su componente resistiva (Huigen et al 2002).

\subsection{Señales biomédicas}

Existen distintas señales bioléctricas de interés biomédico, cada una con características propias debidas al tipo de células que las originan, su ubicación, proceso o función asociada. Las más utilizadas con fines diagnóstico son ECG (producida por el corazón), EEG (generada por distintas zonas de la corteza cerebral), EMG (originada en los músculos estriados) y EOG (potencial entre cornea y retina). Las amplitudes y anchos de banda típicos de estas señales se indican en la Figura 2-3. 


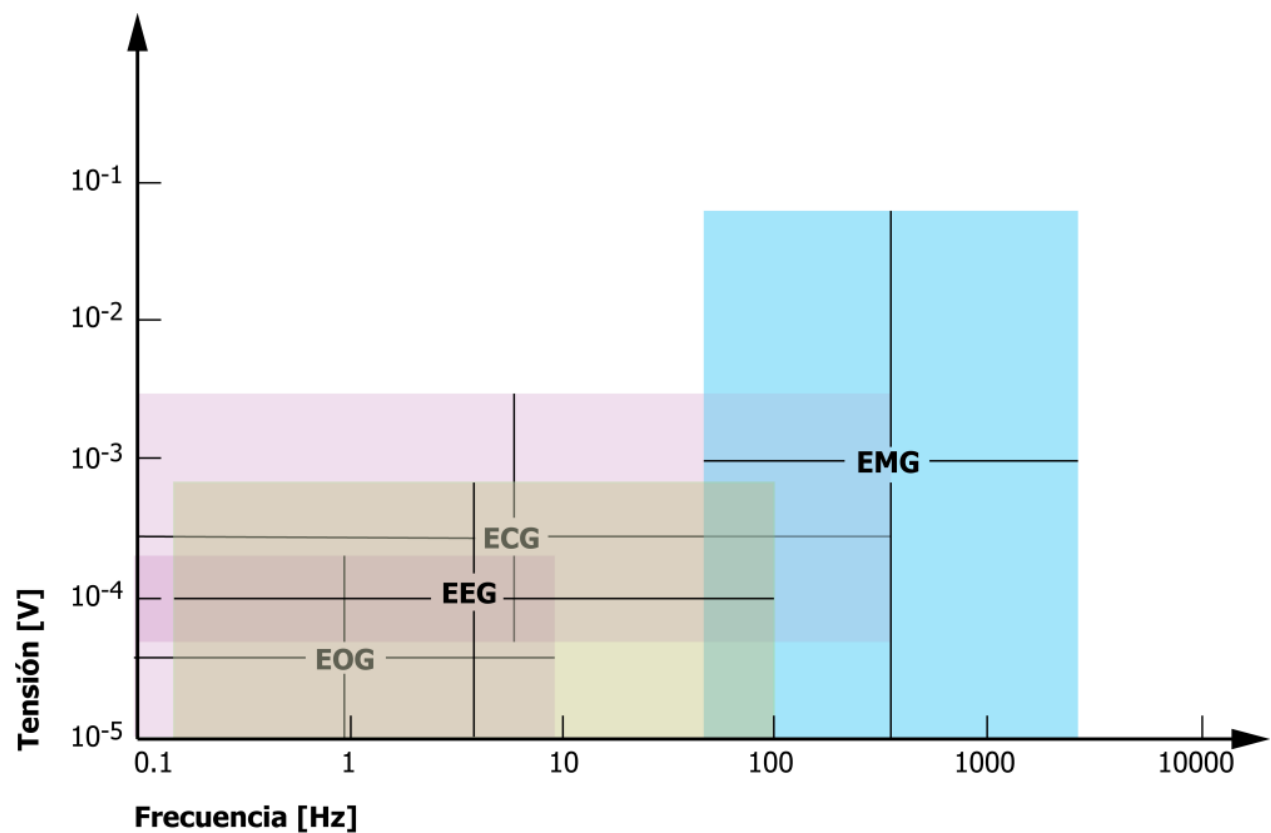

Figura 2-3: Rangos de frecuencia y tensión para algunos de los biopotenciales más comunes. (Figura tomada y modificada de Neuman (2009b))

Los biopotenciales más utilizados para implementar DAs son el EEG y el EMG. Los primeros permiten detectar la voluntad del usuario directamente desde su cerebro, mientras que el EMG lo hace a partir de la actividad eléctrica de los músculos. En líneas generales, los DA basados en EMG permiten alcanzar las mayores velocidades de comunicación, pero requieren que el camino neural desde el cerebro hasta el músculo "funcione". Las interfaces basadas en EEG pueden operar aun cuando nervios y músculos estén dañados o presenten patologías, pero sus tasas de transferencia son bajas. Una alternativa interesante son las interfaces hibridas (Leeb et al 2011, MüllerPutz 2011) que combinan ambos tipos de señales. Así, puede utilizarse como primera opción EMG, y cuando el usuario se fatiga, y ya no es posible operar en este modo, pasar a utilizar EEG resignando velocidad pero manteniendo la comunicación. Este tipo de DA muestra con claridad que tanto el EMG como EEG son señales útiles, pero que tienen características propias, que definen su aplicabilidad y el tipo de paradigma a utilizar.

\subsubsection{Electroencefalograma (EEG)}

Las señales de EEG son producidas por la corteza cerebral, y proveen la conexión más directa con la voluntad del usuario de un DA. Cuando son captadas en la superficie del cuero cabelludo, sus amplitudes son de entre $1 \mu \mathrm{V}_{\mathrm{pp}}$ y $500 \mu \mathrm{V}_{\mathrm{pp}}$, y su ancho de banda se extiende desde 0 a $100 \mathrm{~Hz}$ aproximadamente.

La información presente en el EEG depende fuertemente de la ubicación de los electrodos, debido a la especialización funcional que presentan las distintas regiones de la corteza cerebral. Tanto la forma de onda como el contenido espectral varían de acuerdo a las acciones del sujeto, haciendo posible obtener información sobre sus actividades y sobre su voluntad. 
La riqueza de información que contienen las señales de EEG estimuló el desarrollo de varios paradigmas de Interfaces Cerebro-Computadora (ICC o BCl: Brain Computer Interface), que se diferencian entre sí en cuanto a la estrategia mental utilizada para producir los potenciales que reflejen la voluntad del usuario (Graimann et al 2010). Las señales de EEG más utilizadas en $\mathrm{BCI}$ son:

- Ritmos cerebrales. Son formas de onda cuasi periódicas identificadas en diferentes bandas de frecuencia. En general estos ritmos tienen una localización particular en la corteza cerebral, asociada con una función determinada y su amplitud puede aumentar (sincronización) o disminuir (desincronización) de acuerdo a las actividades mentales que realiza la persona.

- El ritmo alfa (a) engloba las oscilaciones de entre 8 y $13 \mathrm{~Hz}$ de la región occipital, asociada al procesamiento visual. Se observa su sincronización al relajar la vista o cerrar los ojos y su desincronización con la atención visual.

- El ritmo mu $(\mu)$ se refiere a las oscilaciones que, en la misma banda de frecuencia que el ritmo a, se desarrollan sobre la corteza motora primaria. Distintos estudios demuestran su desincronización al realizar un movimiento e incluso al imaginarlo (McFarland et al 2000, Pfurtscheller y Neuper 1997).

- El ritmo beta $(\beta)$ está comprendido entre 13 y $30 \mathrm{~Hz}$. Cuando se observa en las regiones central y frontal de la corteza, se asocia a estados de concentración mental. Este ritmo también está relacionado con la realización de movimientos, cuando es captado sobre la corteza motora, mostrando desincronización durante la ejecución del mismo y resincronización al finalizar el movimiento (Pfurtscheller y Neuper 2002).

- P300. Es un tipo de onda de EEG que se capta en la corteza centro-parietal. Se manifiesta como una desviación positiva de la señal, que ocurre entre 250 y $300 \mathrm{~ms}$ luego de percibirse un estímulo poco probable, deseado o significante (Wolpaw y Boulay, 2002). Si bien el estímulo puede ser visual, auditivo o somatosensorial (táctil), el estímulo más utilizado en $\mathrm{BCl}$ es de tipo visual. En el paradigma más usual, se disponen en una matriz bidimensional distintas opciones que destellan aleatoriamente; cuando el usuario de la $\mathrm{BCl}$ desea una opción, la observa e idealmente, cuando ésta destella, se producirá una onda P300 que puede ser detectada. La relación señal a ruido de las señales capturadas en general requiere promediar varias iteraciones (trials) para conseguir una detección de buena calidad.

- Potenciales evocados visuales de estado estacionario (SSVEP: Steady State Visual Evoked Potentials). Son ondas de EEG, captadas sobre la corteza visual, que se originan a partir de la observación de estímulos visuales que destellan a un frecuencia fija. Los SSVEP se caracterizan por tener la misma frecuencia fundamental que el estimulo. De este modo es posible mostrar al usuario un conjunto de opciones, donde cada una posee una fuente de luz que destella a distinta frecuencia y, mediante el procesamiento espectral, puede determinar que opción observa la persona (García et al 2011). 
(a)

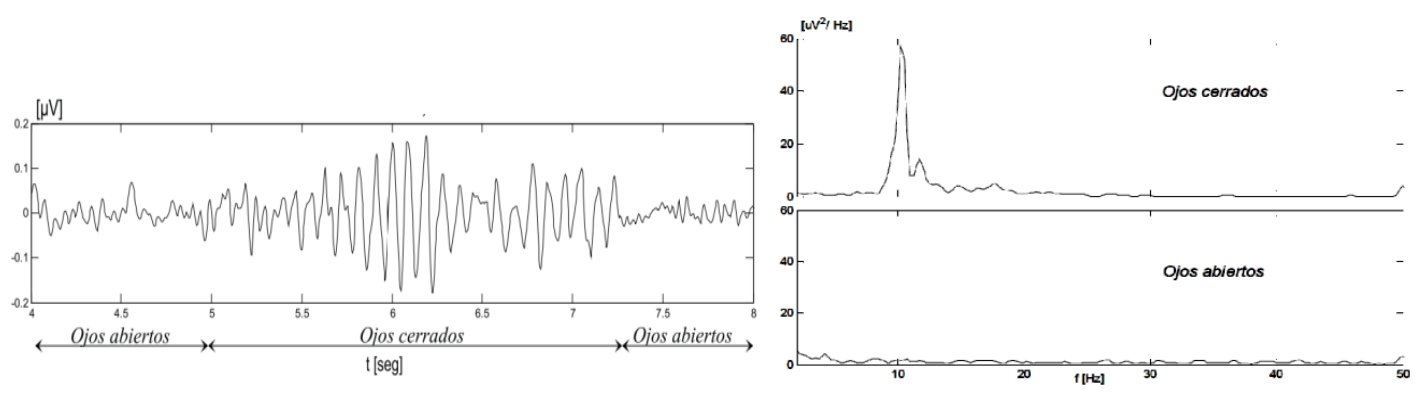

(b)

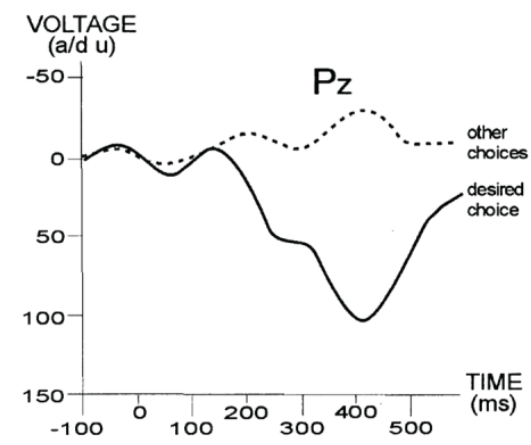

Figura 2-4: (a) Señal característica del ritmo alfa al cerrar los ojos. Izquierda: evolución temporal. Derecha: Localización espectral (b) Señal característica del pulso P300. (en línea continua) cuando se ilumina el símbolo deseado en una $\mathrm{BCl}$ con distintos objetivos visuales que destellan aleatoriamente. En línea punteada se grafica la señal obtenida tras el destello de un símbolo no deseado por el usuario (Figura Tomada de Wolpaw et al (2002b))

\subsubsection{Electromiograma (EMG)}

La señal de EMG es la manifestación eléctrica de la activación neuromuscular asociada con una contracción, la cual es producida por potenciales de acción que llegan al músculo a través de un nervio. Los músculos están formados por conjuntos de fibras musculares que son inervados por una misma neurona motora. A este conjunto de lo denomina unidad motora (MU: Motor Unit).

El potencial de acción proveniente de la neurona motora ingresa al músculo por la juntura neuromuscular, que es la región de sinapsis entre las terminaciones del axón de la neurona motora y las fibras musculares de la MU. Allí se inicia la despolarización de las membranas de las fibras musculares, que producirán la contracción. La actividad eléctrica que emana de la activación de una MU, y que arriba a la superficie de los electrodos se denomina potencial de acción de unidad motora (MUAP: Motor Unit Action Potential). Este representa una forma de onda característica, que depende también de la geometría y de posición de los electrodos. En la Figura 2-5 se ilustra como las distintas fibras musculares aportan a la constitución del MUAP. La señal de EMG es una suma ponderada de MUAPs, que pueden considerarse sus "unidades constitutiva" o sus "átomos". Las características del EMG dependen, entre otras cosas, del patrón de reclutamiento de las distintas MU y de las tasas de disparo de cada una. (De Luca, 2006).

La manifestación eléctrica del MUAP está acompañada de un impulso contráctil de las fibras motoras. Para que esta contracción sea sostenida en el tiempo, las MUs deberán activarse repetidamente. Por lo tanto, durante una contracción muscular, la señal de EMG observada será la composición de distintos trenes de MUAPs. La 
producción de fuerza en el músculo obedece a dos mecanismos principales: el reclutamiento de MUs y el aumento de la tasa de disparo de las MUs (Farina et al 2004). Esto se refleja en la señal de EMG que, como fue dicho, es la suma de trenes de MUAPs, y por lo tanto habrá una relación entre distintas características del EMG y la fuerza realizada por el músculo. En la Figura 2-6 se aprecia dicha relación entre fuerza y valor RMS de la señal (normalizados ambos respecto del máximo valor), para tres músculos diferentes (De Luca 1997).

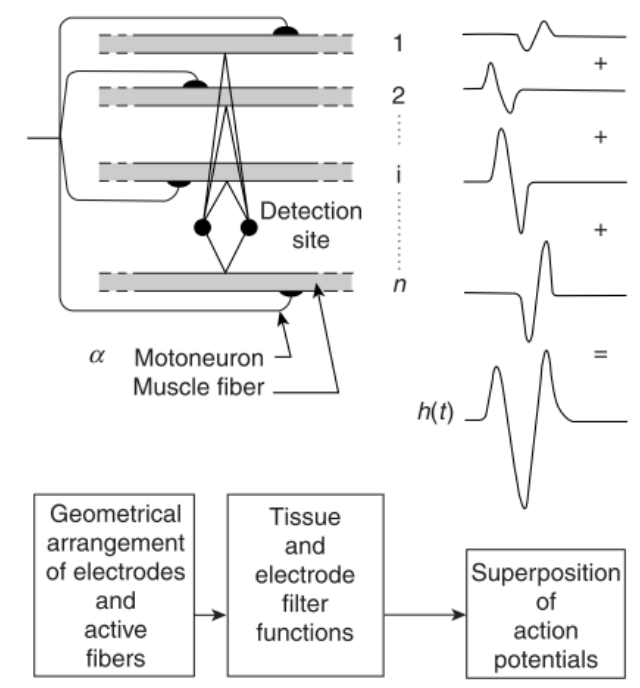

Figura 2-5: Esquema de la generación de un MUAP. El mismo será una superposición de los potenciales de acción de las distintas fibras que componen una misma unidad motora. Se puede observar que la forma de onda resultante $h(t)$ dependerá de la ubicación relativa del par de electrodos respecto de cada una de las fibras, así también dependerá de la distancia inter-electrodo y de la geometría de los mismos. (Figura tomada de De Luca 2006).

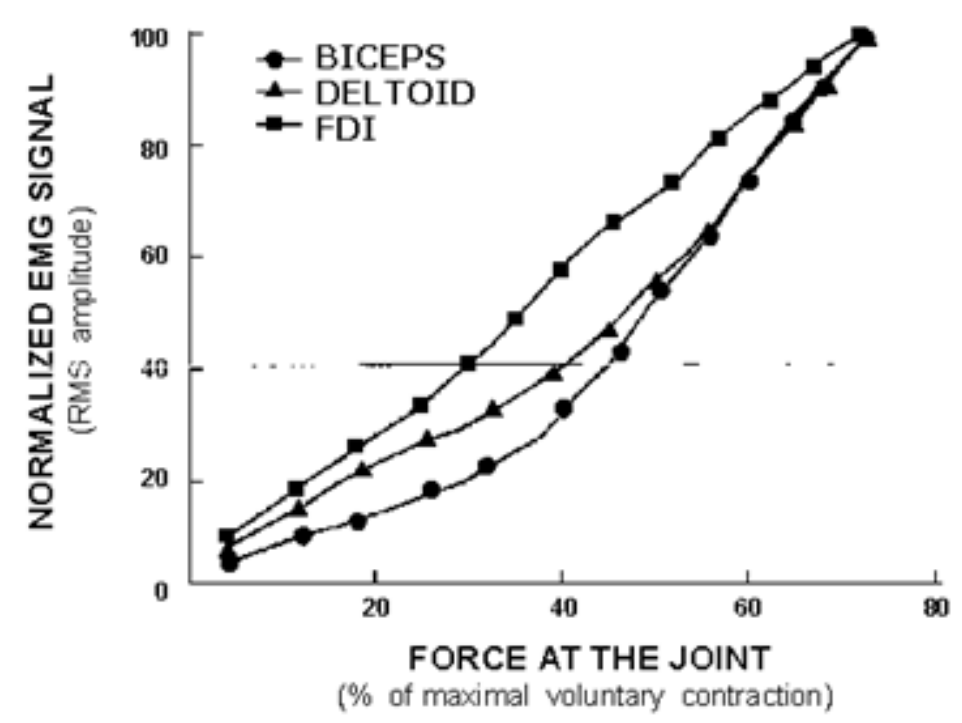

Figura 2-6: Relación entre fuerza y valor RMS (amplitud) de la señal de EMG para tres músculos diferentes. Se muestran ambos ejes normalizados respecto de su máximo valor. (Figura tomada y modificada de De Luca 1997) 
El EMG superficial es una composición de los MUAPs provenientes de MUs aledañas a los electrodos, que pueden pertenecer a distintos músculos produciendo un fenómeno que se denomina crosstalk. Mediante la utilización de arreglos lineales o matriciales de electrodos, es posible discernir entre señales provenientes de distintos músculos, consiguiendo mejorar la selectividad hacia un determinado músculo y/o dirección de propagación (Merletti et al 2009, De Luca 1997).

Las señales de EMG son una muy buena vía para manifestar la voluntad del usuario y controlar una interfaz Hombre-Máquina. En la medida que se tenga control voluntario sobre un músculo, la persona podrá modular a voluntad la amplitud (o algún otro parámetro) de la señal de EMG, y esto podrá ser detectado a partir de esta señal. En las Figura 2-7 se muestra un registro de EMG realizado sobre el músculo facial cigomático mayor izquierdo de una persona con esclerosis lateral amiotrófica (ELA), mientras que en la Figura 2-8 se muestra un registro del músculo bíceps brachii de un sujeto sano. Nótese la diferencia de amplitud y morfología en la señal de EMG para ambos casos. Estas diferencias sustanciales deben tenerse en cuenta en el diseño de DAs controlados por EMG.

(a)

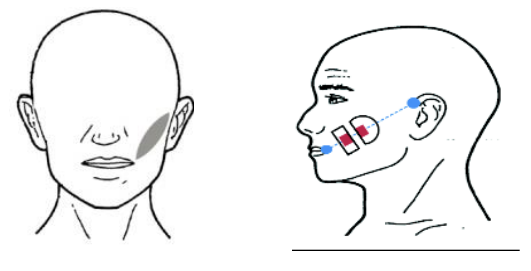

(b)
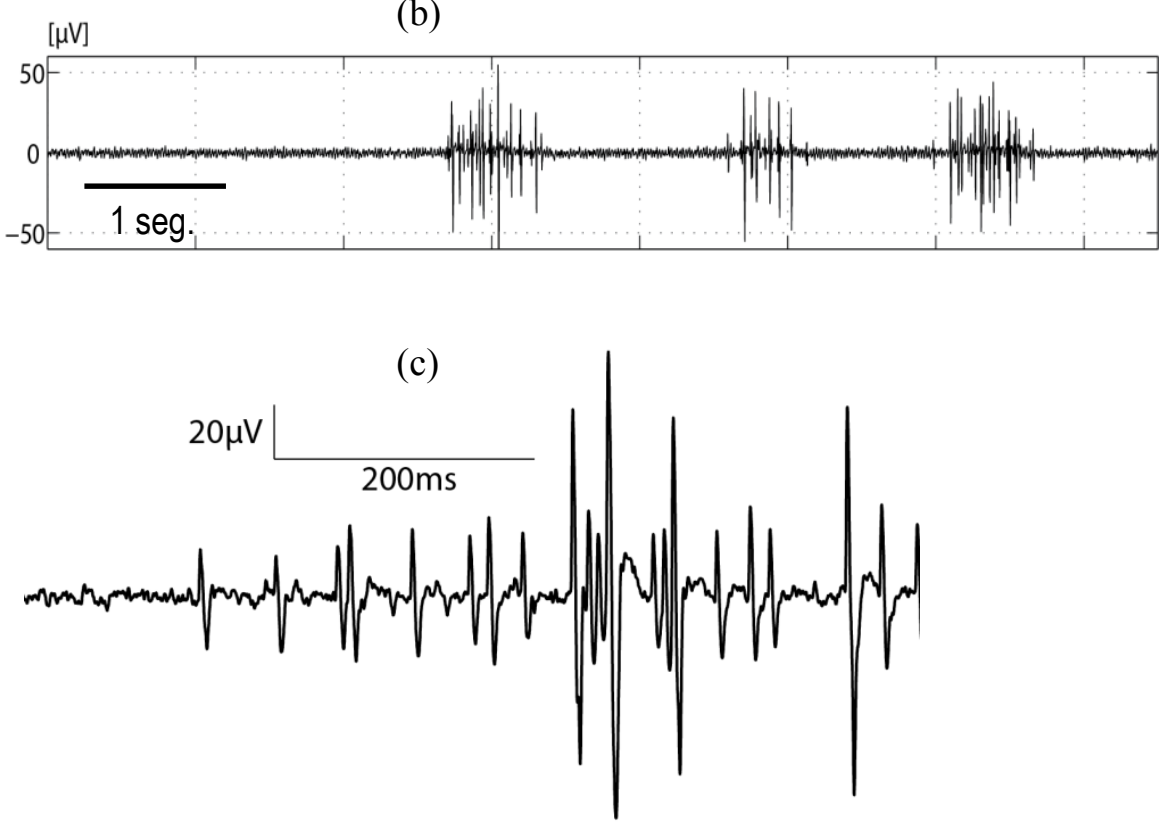

Figura 2-7: (a) Ubicación del músculo facial cigomático mayor izquierdo y posicionamiento de los electrodos en rojo. (b)Señal cruda de EMG superficial del músculo cigomático mayor izquierdo, donde se observan claramente tres contracciones. (c) Detalle del EMG al comienzo de una contracción, donde es posible observar con claridad los MUAPS individuales. 
(a)

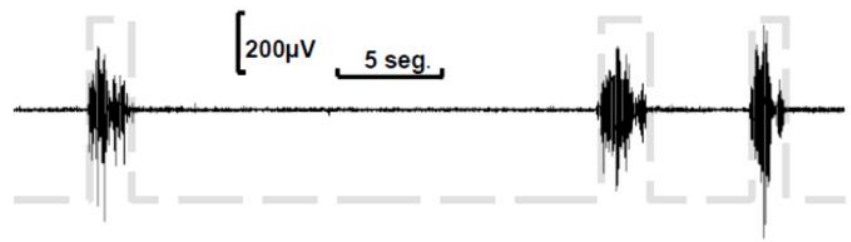

( (b)

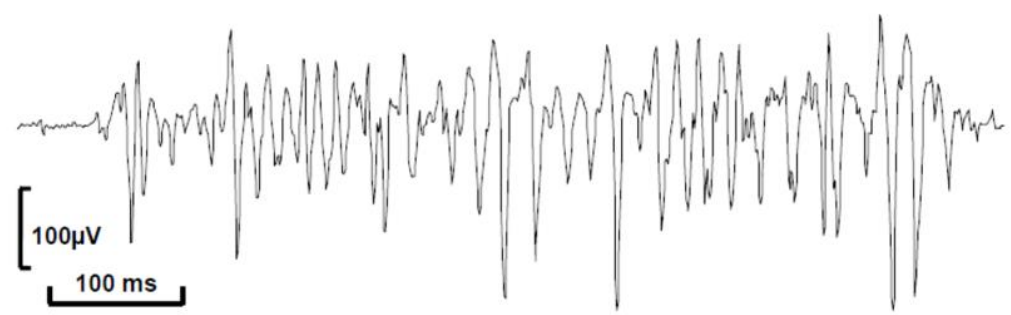

Figura 2-8: (a) Señal cruda de EMG superficial del músculo bíceps brachii, donde se indican en gris las contracciones. (b) detalle del inicio de una de las contracciones donde no llegan a identificarse las MUAPs individualmente.

\subsubsection{Amplificador de biopotenciales}

Las señales de interés (EEG, EMG) son diferencias de tensión entre distintos puntos de la superficie del cuerpo. La función de un amplificador de biopotenciales ( $A B$ ), es acondicionarlas para que puedan presentarse al Conversor Analógico-Digital (ADC: Analog to Digital Converter). Un AB recibe señales de reducida amplitud (ver Figura 2-3) a través de altas y poco predecibles impedancias de electrodo $Z_{\mathrm{Ei}}$, inmersas en importantes potenciales de continua $V_{\mathrm{Ei}}$; $y$ debe entregarlas con amplitud e impedancias apropiadas para el ADC.

En el caso más típico, un $A B$ es un amplificador diferencial (ver Figura 2-9). En su entrada se presenta una tensión diferencial $v_{\mathrm{DM}}$, asociada con la señal de interés que se desea capturar; y una tensión de modo común $v_{\mathrm{CM}}$, que constituye una perturbación a rechazar. La salida $v_{0}$ del $A B$ dependerá de ambas según:

$$
v_{\mathrm{O}}=G_{\mathrm{DD}} v_{\mathrm{DM}}+G_{\mathrm{DC}} v_{\mathrm{CM}}
$$

siendo $G_{\mathrm{DD}}$ la ganancia de modo diferencial y $G_{\mathrm{DC}}$ la ganancia de modo común del amplificador.

Un factor de mérito de un amplificador diferencial es su Rechazo de Modo Común (CMRR: Common Mode Rejection Ratio), que describe su habilidad de amplificar $v_{\mathrm{DM}}$, y al mismo tiempo rechazar $v_{\mathrm{CM}}$. El CMRR se define como:

$$
C M R R=\frac{G_{\mathrm{DD}}}{G_{\mathrm{DC}}}
$$

y expresado en decibles:

$$
C M R R_{[\mathrm{dB}]}=20 \log _{10}\left(\frac{G_{\mathrm{DD}}}{G_{\mathrm{DC}}}\right)
$$




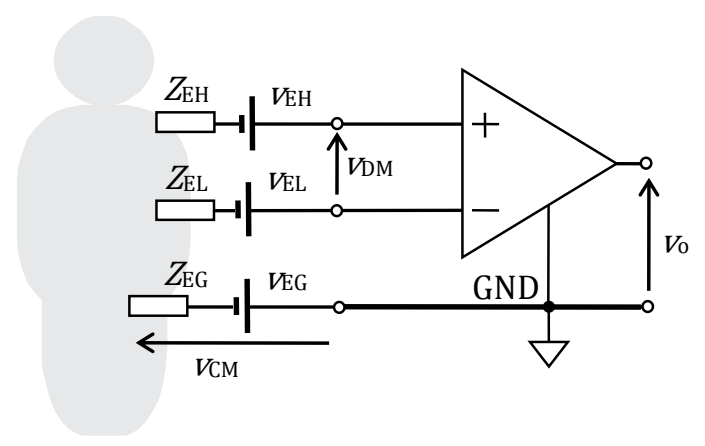

Figura 2-9: Esquema donde se observa el $A B$, las impedancias $\boldsymbol{Z}_{\mathrm{Ei}}$ debidas a los electrodos, así como los potenciales de continua $\boldsymbol{V}_{\mathbf{E i}}$ de de cada uno de estos. Nótese que el tercer electrodo refiere el sistema de potenciales del sujeto al potencial de "masa" o "común" del AB (GND: Ground). La tensión que se desarrolle sobre dicho electrodo será la tensión de modo común $\boldsymbol{v}_{\mathbf{C M}}$.

\subsection{Interferencia Electromagnética en la adquisición de biopotenciales}

La interferencia electromagnética es uno de los principales problemas en la adquisición de biopotenciales. Se trata de señales indeseadas provenientes de fuentes externas, que se acoplan al circuito de medida por distintos mecanismos en los que están implicados campos eléctricos, magnéticos o electromagnéticos. La persona y el $A B$ no se encuentran perfectamente aislados de los potenciales externos como la tensión de línea $\left(V_{\mathrm{PL}}\right)$ y el potencial de tierra, sino vinculados a ellos por distintos tipos de acoplamientos. Por este motivo, el $A B$ no solo registrará en su entrada diferencial la señal biopotencial $v_{\mathrm{S}}$, sino también una tensión de interferencia $v_{\mathrm{EMI}}$ (ver Figura 2-10):

$$
v_{\mathrm{DM}}=v_{\mathrm{S}}+v_{\mathrm{EMI}}
$$

Adicionalmente, ya dentro del $A B$, se sumará una componente debida a la transformación de modo común a modo diferencial dado que el CMRR del $A B$ real no es infinito. En la Figura 2-10 se puede observar cómo se puede modelar al $\mathrm{AB}$, como un $\mathrm{AB}$ ideal con rechazo de modo común infinito, cuya entrada diferencial $v_{\mathrm{i}}$ registrará la señal de modo diferencial $v_{\mathrm{DM}}$ en serie con un generador de valor $\frac{v_{\mathrm{CM}}}{C M R R_{\mathrm{AB}}}$, que da cuenta de su CMRR

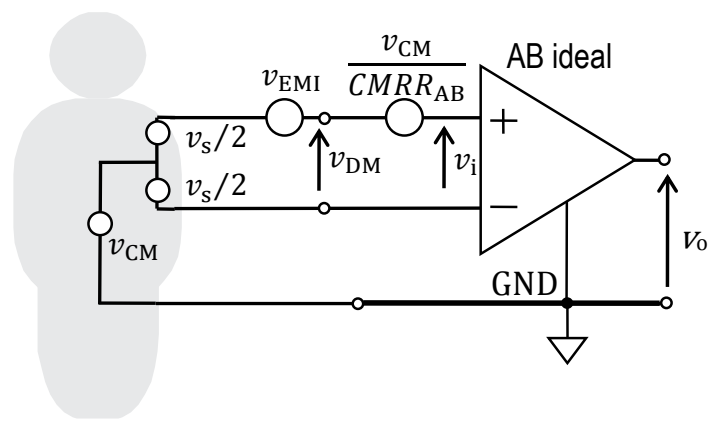

Figura 2-10: Esquema que muestra la tensión de interferencia modelada como una fuente $\boldsymbol{v}_{\text {EMI }}$ en serie con el potencial biológico $\boldsymbol{v}_{\mathbf{S}}$. Adicionalmente se suma la componente $\boldsymbol{v}_{\mathrm{CM}} / \boldsymbol{C} \boldsymbol{M} \boldsymbol{R} \boldsymbol{R}_{\mathrm{AB}}$ producida por la transformación de modo común a diferencial.

La principal fuente de EMI es la red de alimentación de energía eléctrica ( $220 \mathrm{Vrms}$ y $50 \mathrm{~Hz}$ en Argentina). Como se trata de una fuente de EMI de baja frecuencia, con una longitud de onda mucho mayor que las dimensiones del sistema $\left(\lambda_{50 \mathrm{~Hz}}=6000 \mathrm{~km}\right)$, sus efectos pueden asociarse a acoplamientos capacitivos (corrientes de 
desplazamiento) y acoplamientos magnéticos (fem inducidas). Los mecanismos de interferencia quedan razonablemente bien descriptos mediante el modelo de EMI (Spinelli 2007, Metting Van Rijn et al. 1990) presentado en la Figura 2-11. Este modelo considera al cuerpo humano como un volumen conductor (con conductividad finita), con una capacidad $\left(C_{\mathrm{P}}\right)$ respecto de los conductores de la línea y una capacidad $\left(C_{\mathrm{B}}\right)$ respecto del potencial de "tierra". Un fenómeno similar ocurre con el $A B$, donde su "masa" o "común", tiene también capacidades de acoplamiento con la línea $\left(C_{\mathrm{SUP}}\right)$ y con el potencial de tierra $\left(C_{\mathrm{ISO}}\right)$. Cualquier cuerpo en las cercanías de la línea de distribución de energía y el plano de tierra presentará este tipo de capacidades, por ejemplo los cables de conexión al paciente también se vinculan con la línea de tensión y con tierra mediante las capacidades $C_{\mathrm{Li}}$ y $C_{\mathrm{Ti}}$, respectivamente (ver Figura 2-11). Para analizar los efectos de EMI en la señal finalmente capturada por el $A B$, es necesario también incluir en el modelo sus impedancias de entrada para modo diferencial $Z_{\mathrm{D}}$ y para modo común $Z_{\mathrm{Ci}}$.

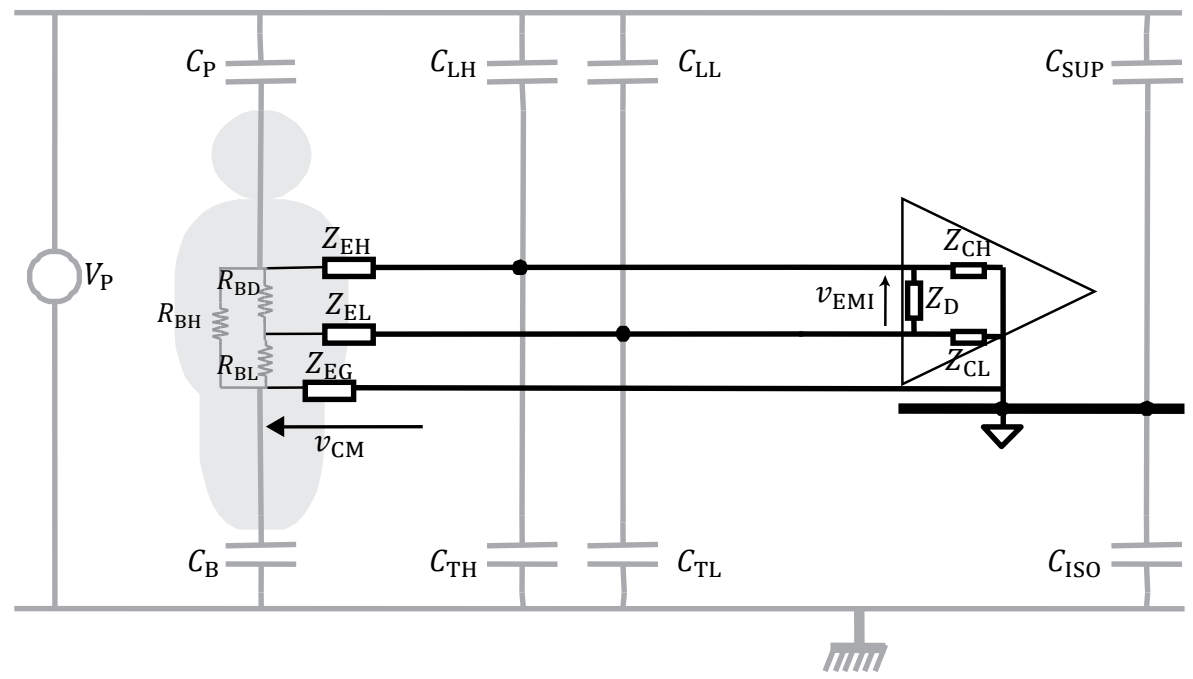

Figura 2-11: Modelo de EMI con parámetros concentrados. El mismo permite describir y predecir los fenómenos de interferencia por acoplamiento capacitivo.

Si se considera que no existen biopotenciales $\left(v_{\mathrm{S}}=0\right)$, la tensión $v_{\mathrm{i}}$ que registraría el sistema de adquisición, se deberá exclusivamente a los mecanismos de interferencia y estará dada por:

$$
v_{\mathrm{i}}=v_{\mathrm{EMI}}+\frac{v_{\mathrm{CM}}}{C M R R_{\mathrm{AB}}}
$$

Siendo $v_{\mathrm{EMI}}$ y $v_{\mathrm{CM}}$ las tensiones de EMI de modo diferencial y modo común a la entrada del $\mathrm{AB}$. Para calcular $v_{\mathrm{EMI}}$ y $v_{\mathrm{CM}}$ se debería resolver el circuito completo de la Figura 2-11, pero la expresión resultaría muy compleja, y no permitiría visualizar con claridad los aportes de los distintos mecanismos de interferencia. Por esto, se analizarán los diferentes mecanismos por separado. Si bien no se llega a una expresión exacta, pues no se aplicará el principio de superposición sino modelos parciales, se consigue una mejor comprensión que permite obtener una idea del orden de magnitud de los niveles de interferencia esperables. 


\subsubsection{Mecanismos de Interferencia que producen $v_{C M}$}

La tensión $v_{\mathrm{CM}}$ se produce porque el denominado electrodo de referencia (o tercer electrodo) tiene una impedancia $Z_{\mathrm{EG}}$ no nula, y no logra imponer la tensión de referencia (masa del $\mathrm{AB}$ ) como tensión del sujeto. En la Figura 2-12 se observa una versión simplificada del modelo EMI de la Figura 2-11, donde se desprecia el aporte del acoplamiento a los cables de electrodo en la generación de $v_{\mathrm{CM}}$. Si además se tiene en cuenta que las impedancias de entrada de modo común del $A B$ son mucho mayores que las impedancias de electrodo, se puede predecir el valor de $v_{\mathrm{CM}}$ a partir de $C_{\mathrm{B}}, C_{\mathrm{P}}, C_{\mathrm{ISO}}, C_{\mathrm{SUP}}$ y $Z_{\mathrm{EG}}$, según la fórmula (2-6).

$$
v_{\mathrm{CM}}=i_{\mathrm{EG}} \cdot Z_{\mathrm{EG}}=\frac{V_{\mathrm{PL}} \cdot\left|\frac{C_{\mathrm{P}}}{C_{\mathrm{P}}+C_{\mathrm{B}}}-\frac{C_{\mathrm{SUP}}}{C_{\mathrm{SUP}}+C_{\mathrm{ISO}}}\right|}{\sqrt{\left[\left(\frac{1}{C_{\mathrm{P}}+C_{\mathrm{B}}}+\frac{1}{C_{\mathrm{SUP}}+C_{\mathrm{ISO}}}\right) \frac{1}{2 \pi f_{\mathrm{PL}}}\right]^{2}+Z_{\mathrm{EG}}^{2}}} Z_{\mathrm{EG}}
$$

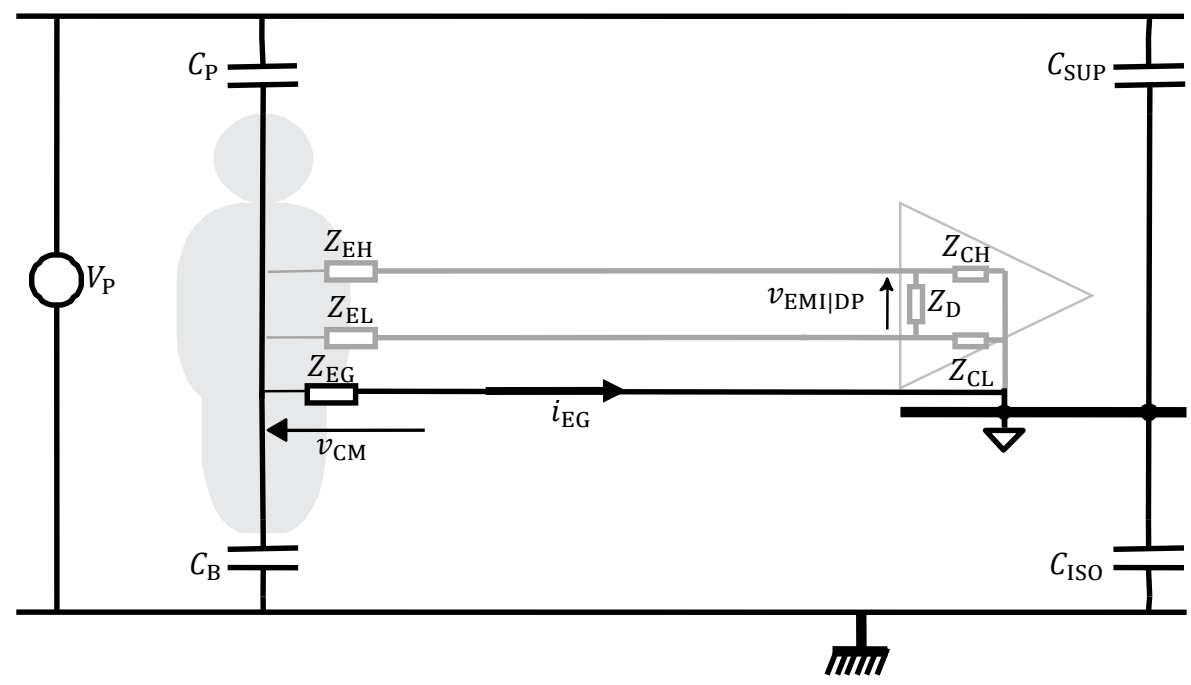

Figura 2-12: Modelo de EMI simplificado que explica la formación de una tensión de Modo Común en el sistema. Nótese que $\boldsymbol{v}_{\mathbf{C M}}$ depende solo de las capacidades de acoplamiento $\boldsymbol{C}_{\mathrm{P}}, \boldsymbol{C}_{\mathrm{B}}, \boldsymbol{C}_{\mathrm{SUP}}$ y $\boldsymbol{C}_{\mathrm{ISO}}$, y de la impedancia del electrodo de referencia $\boldsymbol{Z}_{\mathrm{EG}}$. En el caso ideal de que fuese nula, la tensión de modo común $\boldsymbol{v}_{\mathrm{CM}}$ también lo sería. Se observa la tensión de interferencia $\boldsymbol{v}_{\text {EMI|DP }}$ a la entrada del $A B$ producida por el efecto divisor de potencial.

Si bien esta tensión es de modo común, debido a transformaciones de modo que ocurren tanto dentro del $A B$ como en la red formada por las impedancias de entrada del $A B$ y las impedancias de electrodo (efecto divisor de potencial), pueden producirse tensiones de modo diferencial que se superpondrán al biopotencial de interés (Spinelli 2007, Metting Van Rijn et al. 1990, Huhta y Webster 1973). 


\subsubsection{Mecanismos de Interferencia que producen $v_{D M}$}

\subsubsection{Tensión $v_{\mathrm{DM}}$ producida por transformación de $v_{\mathrm{CM}}$.}

La tensión $v_{\mathrm{CM}}$ entre el paciente y la masa del $\mathrm{AB}$ producirá una tensión de modo diferencial $v_{\mathrm{EMI}}$ debido a desbalances entre las impedancias de cada electrodo $Z_{\mathrm{Ei}}$ y las impedancias de entrada de modo común $Z_{\mathrm{Ci}}$ del $A B$ (ver Figura 2-12). Estás impedancias definen dos divisores de tensión, razón por la cual este mecanismo se conoce como efecto divisor de potencial (Huhta y Webster 1973). La tensión $\left.v_{\text {EMI }}\right|_{\text {DP }}$ que produce estará dada por. Considerando como única fuente de tensión a $v_{\mathrm{CM}}$, la tensión $\left.v_{\mathrm{EMI}}\right|_{\mathrm{DP}}$ está dada por':

$$
\left.v_{\mathrm{EMI}}\right|_{\mathrm{DP}}=v_{\mathrm{CM}}\left(\frac{Z_{\mathrm{CH}}}{Z_{\mathrm{CH}}+Z_{\mathrm{EH}}}-\frac{Z_{\mathrm{CL}}}{Z_{\mathrm{CL}}+Z_{\mathrm{EL}}}\right)
$$

Definiendo la impedancia de electrodo promedio $\overline{Z_{\mathrm{E}}}$ y la diferencia de impedancias $\Delta Z_{\mathrm{E}}$,

$$
\overline{Z_{E}}=\frac{Z_{\mathrm{EH}}+Z_{\mathrm{EL}}}{2} ; \Delta Z_{\mathrm{E}}=Z_{\mathrm{EH}}-Z_{\mathrm{EL}}
$$

Asumiendo que en general las impedancias de entrada de modo común están bien balanceadas entre sí $\left(Z_{\mathrm{CH}}=Z_{\mathrm{CL}}=Z_{\mathrm{C}}\right)$, y que el valor de las mismas es mucho mayor que las impedancias de electrodo $\left(\left|Z_{\mathrm{Ci}}\right| \gg\right.$ $\left.\left|Z_{\mathrm{Ei}}\right|\right)$, resulta:

$$
\left.v_{\mathrm{EMI}}\right|_{\mathrm{DP}} \cong v_{\mathrm{CM}} \frac{Z_{\mathrm{C}}}{\Delta Z_{\mathrm{E}}}=v_{\mathrm{CM}} \frac{1}{C M R R_{\mathrm{DP}}}
$$

Es interesante comparar este efecto con la transformación de modo producida por el CMRR del propio $A B$, explicita en el segundo término de la ecuación (2-5):

$$
\left.v_{E M I}\right|_{\mathrm{AB}} \cong v_{C M} \frac{1}{C M R R_{\mathrm{AB}}}
$$

Considerando un $\Delta Z_{\mathrm{E}}$ de unos $50 \mathrm{k} \Omega$ a $50 \mathrm{~Hz}$, y una $Z_{\mathrm{C}}$ de $200 \mathrm{M} \Omega$ (corresponden a $15 \mathrm{pF}$ a $50 \mathrm{~Hz}$ ), el factor $Z_{\mathrm{C}} / \Delta Z_{\mathrm{E}}$ en (2-9) es de aproximadamente $1 / 4000$, que equivale a un $C M R R_{\mathrm{DP}}$ de unos $70 \mathrm{~dB}$. Ambos factores de rechazo de modo común pueden reunirse en un CMRR efectivo (Huhta y Webster 1973, Winter y Webster 1983):

$$
C M R R_{\mathrm{E}}=\left[\frac{1}{C M R R_{\mathrm{AB}}}+\frac{1}{C M R R_{\mathrm{DP}}}\right]^{-1}
$$

Esto indica que un $\mathrm{AB}$ con un $C M R R_{\mathrm{AB}}$ de $90 \mathrm{~dB}$ es suficiente para que su efecto no sea percibido. Es decir, los altísimos valores de $C M R R_{\mathrm{AB}}$ de los amplificadores de instrumentación en nada ayudan frente al efecto divisor de potencial.

Para reducir el efecto de $v_{\mathrm{CM}}$ existen dos caminos posibles: reducir las impedancias de electrodo preparando la piel o reducir $v_{\mathrm{CM}}$ utilizando circuitos activos reductores de modo común. Esto último será tratado en el Capítulo 4.

\footnotetext{
${ }^{1}$ Como se demuestra en (Pallás-Areny y Webster 1999), la impedancia de modo diferencial $Z_{\mathrm{D}}$ no tiene efecto en esta transformación de modo.
} 


\subsubsection{Corrientes que retornan a tierra pasando por los electrodos; debidas a acoplamiento capacitivo entre la línea y los cables de conexión.}

Las capacidades $C_{L i}$ modelan el acoplamiento por campo eléctrico entre la línea de energía y los cables de los electrodos. Las corrientes de desplazamiento que circulan a través de estas capacidades continúan su recorrido hasta tierra, circulando por las impedancias de electrodos $Z_{\mathrm{E}}$ y la capacidad $C_{\mathrm{B}}$ (ver Figura 2-13).

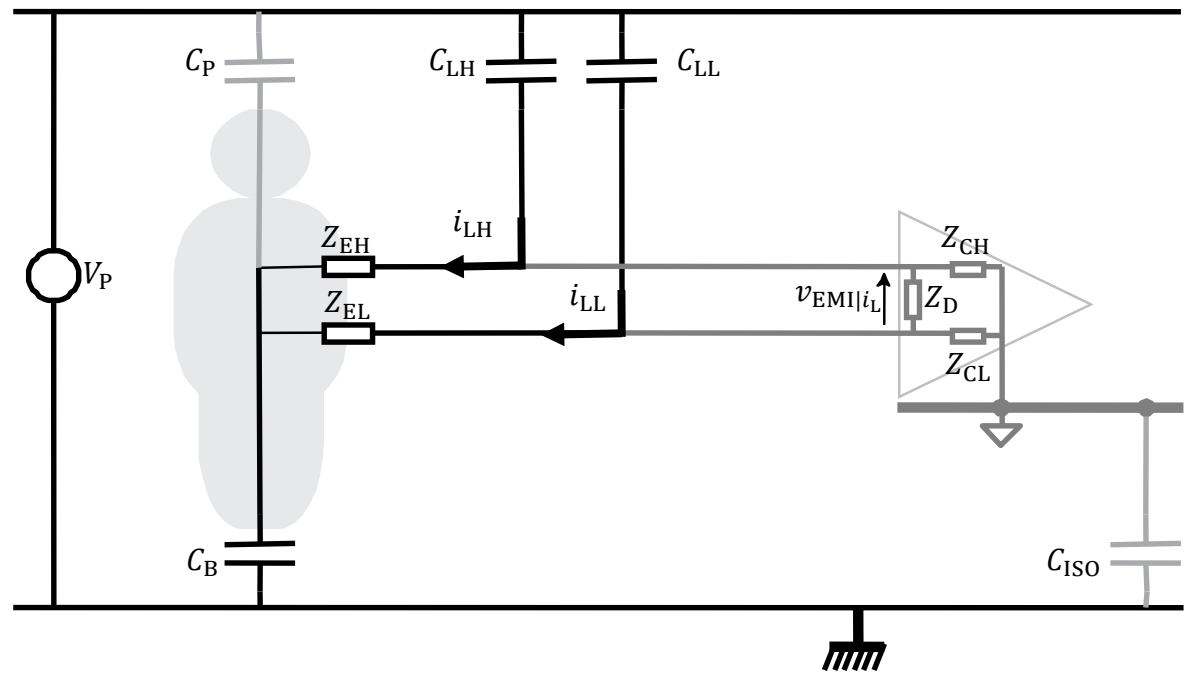

Figura 2-13: Modelo parcial que explica la generación de una tensión diferencial de EMI $\boldsymbol{v}_{\text {EMI|DP }}$ debido al acoplamiento de corrientes de desplazamiento $\boldsymbol{i}_{\mathbf{L H}}$ y $\boldsymbol{i}_{\mathbf{L L}}$ en los cables de electrodo.

Todos los cables de electrodo presentan capacidades similares con la línea siempre que se ubiquen cercanos entre si, por lo tanto las corrientes que llegan a cada cable y pasan a través de los respectivos electrodos son similares. Las altas impedancias asociadas a estas capacidades a $50 \mathrm{~Hz}$, frente a las impedancias de los electrodos $Z_{\mathrm{E} i}$ y a la capacidad $C_{\mathrm{B}}$; hacen que ellas determinen el valor de la corriente circulante. En el caso que la diferencia de potencial entre el cable de electrodo y la línea es igual a la tensión de red esta corriente será: $i_{\mathrm{L}} \cong V_{\mathrm{PL}} \cdot \omega_{\mathrm{PL}} \cdot C_{\mathrm{L}}$. Si bien esta situación no es posible por cuestiones de seguridad, pues implica conectar la persona a tierra, permite estimar el orden de magnitud de la tensión diferencial a la entrada del $A B$ debido al acoplamiento de los cables de electrodos para un caso pesimista.

$$
\left.v_{\mathrm{EMI}}\right|_{\mathrm{L}}=i_{\mathrm{LH}} Z_{\mathrm{EH}}-i_{\mathrm{LL}} Z_{\mathrm{EL}} \approx i_{\mathrm{L}}\left(Z_{\mathrm{EH}}-Z_{\mathrm{EL}}\right)=V_{\mathrm{PL}} \cdot \omega_{\mathrm{PL}} \cdot C_{\mathrm{L}} \cdot \Delta Z_{\mathrm{E}}
$$

En (2-12) se observa que la tensión interferente es directamente proporcional a la diferencia entre las impedancias de los electrodos. Tomando un desbalance de impedancias $\Delta Z_{\mathrm{E}}=50 \mathrm{k} \Omega$ y una capacidad de acoplamiento cable-línea $C_{\mathrm{L}}=0.1 \mathrm{pF}$ (Spinelli, 2007); obtenemos niveles de interferencia que superan en amplitud a las señales de EEG y en la mayoría de los casos a las de EMG: $\left.v_{\mathrm{EMI}}\right|_{\mathrm{i}_{\mathrm{L}}}=0.35 \mathrm{mV}_{\mathrm{RMS}}$.

Una manera de reducir este tipo de interferencias perjudiciales, es blindando los cables de los electrodos y colocando el blindaje a algún potencial fijo 0 a una guarda activa. Esta solución hace que las corrientes que se acoplan al cable circulen solo por el blindaje, retornando a tierra a través de $C_{\text {ISo }} \sin$ pasar por los electrodos. El uso de una guarda activa brinda el mismo efecto, pero sin degradar la impedancia de entrada del amplificador, compensando la capacidad entre el cable y el blindaje.

Otra manera de reducir $\left.v_{\mathrm{EMI}}\right|_{\mathrm{i}_{\mathrm{L}}}$ sin utilizar cables blindados es minimizando la longitud los cables para reducir $C_{\mathrm{L}}$, acercando la etapa de entrada del $\mathrm{AB}$ al sujeto. Esto llevado al extremo resulta en colocar un seguidor de 
tensión sobre el mismo electrodo, requiriendo cables adicionales para su alimentación, lo que les da el nombre de electrodos activos.

\subsubsection{Corrientes que circulan a través del cuerpo; por acoplamiento capacitivo entre la línea y la persona}

Como sucede con el acoplamiento cable-línea, modelado por $C_{\mathrm{LH}}$ y $C_{\mathrm{LL}}$, existe un acoplamiento similar entre el cuerpo del sujeto y la línea de energía eléctrica, modelado por $C_{\mathrm{P}}$ en la Figura 2-14. En este caso, a través de la persona, circulará una corriente $i_{\mathrm{P}}$ determinada por el valor de $C_{\mathrm{P}}$, parte de la cual retornará a tierra a través de $C_{\mathrm{B}}$ (ver Figura 2-14). Al ser el cuerpo humano un conductor de volumen con una conductividad finita, la corriente interna producirá una diferencia de potencial entre los electrodos, dependiendo de los tejidos que atraviese. Considerando dos electrodos de medida, esta situación puede describirse con una resistencia interna $R_{\mathrm{BD}}$. Desde un enfoque conservador, si la totalidad de $i_{\mathrm{P}}$ circulara por $R_{\mathrm{BD}}$, generará una tensión de interferencia diferencial $v_{\mathrm{EMI}} \mathrm{i}_{\mathrm{P}}$ dada por:

$$
\left.v_{\mathrm{EMI}}\right|_{\mathrm{i}_{\mathrm{P}}} \approx \mathrm{i}_{\mathrm{P}} \cdot R_{\mathrm{BD}}=V_{\mathrm{PL}} \cdot \omega_{\mathrm{PL}} \cdot C_{\mathrm{P}} \cdot R_{\mathrm{BD}}
$$

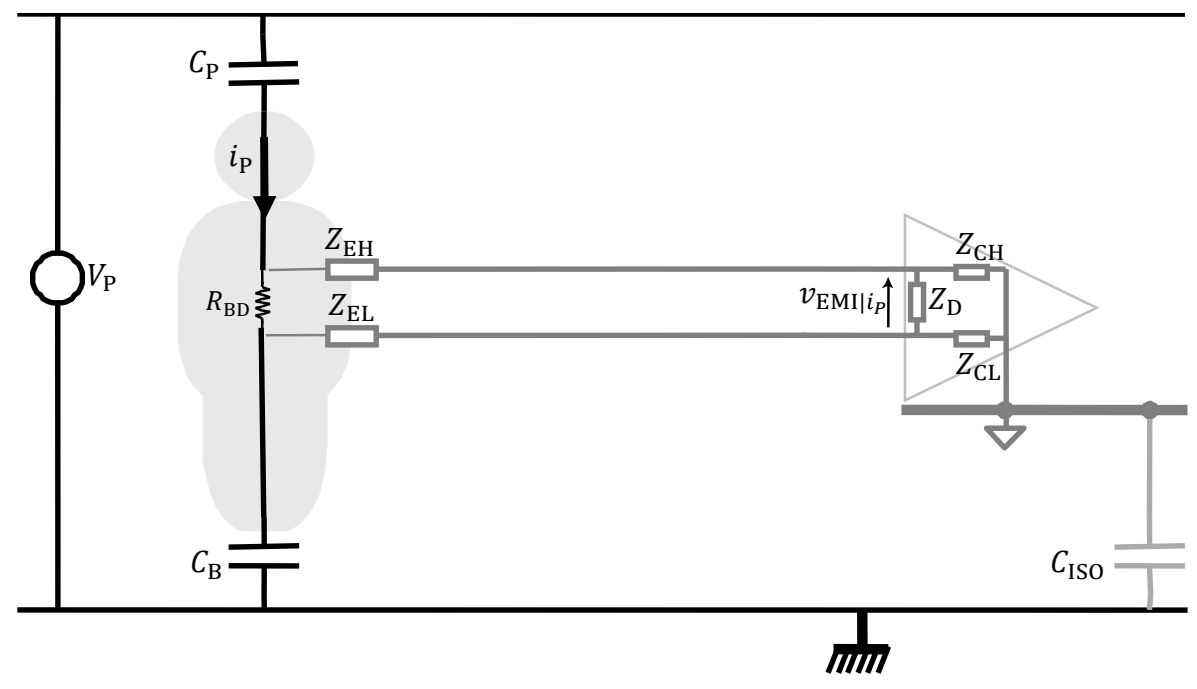

Figura 2-14: Modelo parcial que explica la generación de una tensión diferencial de EMI $\boldsymbol{v}_{\mathbf{E M I} \mid \boldsymbol{i}_{\mathbf{L}}}$ debido al acoplamiento de la corriente de desplazamiento $\boldsymbol{i}_{\mathbf{P}}$ que circula a través del paciente.

Para ilustrar el nivel de EMI que se puede alcanzar por este mecanismo; en un caso pesimista, con valores de capacidad sujeto-línea $C_{\mathrm{P}}=3 \mathrm{pF}$ y resistencia interna $R_{\mathrm{BD}}=500 \Omega$ (valor factible en aplicaciones de $\mathrm{ECG}$ ) se produciría una interferencia diferencial $\left.v_{\mathrm{EMI}, \mathrm{D}}\right|_{\mathrm{i}_{\mathrm{P}}} \approx 100 \mu \mathrm{V}_{\mathrm{RMS}}$. Este valor, se plantea como una cota que difícilmente sea alcanzada en aplicaciones de EEG o EMG, en las cuales los electrodos se encuentran separados por distancias pequeñas, por lo que $R_{\mathrm{BD}}$ será menor. Además, la corriente de desplazamiento $i_{\mathrm{P}}$ ingresa de manera distribuida a lo largo del cuerpo y sólo una fracción de la corriente $i_{\mathrm{P}}$ total aportará a $\left.v_{\mathrm{DM}}\right|_{\mathrm{i}_{\mathrm{P}}}$. En general, los niveles de interferencia alcanzados por circulación interna de corrientes no son de consideración en aplicaciones de EEG y EMG.

\subsubsection{Tensión inducida por acoplamiento magnético en el lazo que forman los cables de electrodos.}

Las corrientes circulantes por la línea y por los cables de alimentación de dispositivos electrónicos generan campos magnéticos variables que inducen fems en los lazos del circuito que atraviesan. El caso más importante 
es el del lazo generado por los cables que unen los electrodos con el $A B$ que representan el lazo de mayor área del sistema y, además la fem inducida se suma directamente a la tensión diferencial a medir (ver Figura 2-15).

$$
\left.v_{\mathrm{EMI}}\right|_{\Phi}=-\frac{\mathrm{d} \Phi}{\mathrm{dt}}=-B . S . \omega_{\mathrm{PL}}
$$

Para valores posibles de campo magnético sinusoidal de $320 \mathrm{nT}$ y $60 \mathrm{~Hz}$ como los ensayados por Hutah y Webster (1973) se obtienen valores de $\left.v_{\mathrm{EMI}}\right|_{\Phi}=100 \frac{\mu \mathrm{V}}{\mathrm{m}^{2}}$. S. Esto significa una interferencia de $10 \mu \mathrm{V}$ para cables de un metro distanciados $10 \mathrm{~cm}$. Es sencillo evitar este tipo de interferencia reduciendo el área $S$ encerrada por los cables de conexión, por ejemplo trenzándolos o utilizando cable plano.

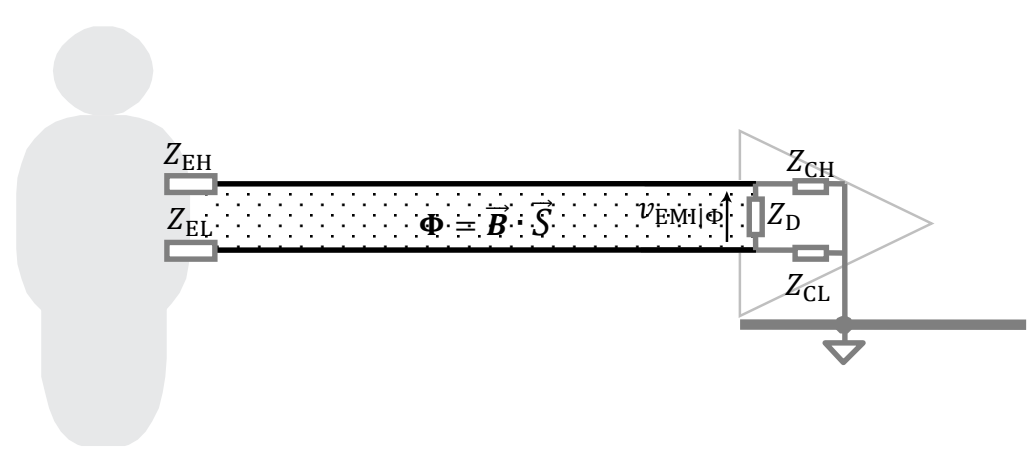

Figura 2-15: Modelo parcial que explica la generación de una tensión diferencial de EMI $\boldsymbol{v}_{\mathrm{EMI} \mid \boldsymbol{\Phi}}$ debido a la fem inducida en el lazo de medida.

\subsection{Estimación de las capacidades parásitas del modelo EMI}

Los dispositivos de ayuda basados en biopotenciales deben funcionar en ambientes no controlados, a diferencia de los laboratorios de investigación y los consultorios médicos; ambientes para los cuales suelen estar pensados los diseños de amplificadores de biopotenciales. Las condiciones de EMI que se imponen en los ambientes domésticos pueden llegar a ser muy agresivas, dado que es imposible para quien diseña, o asiste en el uso de estos equipos, controlar la disposición de fuentes de EMI externas.

Al diseñarse los circuitos de acondicionamiento de señal resulta útil conocer las condiciones de EMI que pueden llegar a imponer el contexto, y así tomar medidas para prevenir perturbaciones en las señales de interés, así como la saturación del amplificador. Las condiciones de EMI quedan principalmente determinadas por el grado de acoplamiento que pueden llegar a tener la persona y el $A B$ a la línea y a tierra, acoplamiento que es representado por las capacidades $C_{\mathrm{B}}, C_{\mathrm{P}}, C_{\mathrm{ISO}}$ y $C_{\mathrm{SUP}}$ del modelo de la Figura 2-11.

Algunos valores típicos de las capacidades de acoplamiento pueden ser encontrados en la literatura, pero se decidió estimar dichos valores bajo distintas condiciones y contextos de uso reales. Esto permite predecir los niveles de interferencia de $\mathrm{MC}$ y brinda información muy valiosa para el diseño del $A B$ y del lazo de realimentación de MC (circuito Driven-Right-Leg) que se describirá en Capítulo 4.

\subsubsection{Método utilizado para estimación de capacidades parásitas}


El método para estimar $C_{\mathrm{B}}$ y $C_{\mathrm{P}}$ (Pallàs-Areny y Colominas 1991) consiste en conectar un resistor $R_{\mathrm{L}}$ de valor variable entre el paciente y tierra (en paralelo con $C_{\mathrm{B}}$ ) y medir la tensión desarrollada sobre el mismo como se muestra en la Figura 2-17. Esta tensión estará dada por:

$$
V_{\mathrm{L}}=\frac{V_{\mathrm{P}} 2 \pi f_{\mathrm{PL}} C_{\mathrm{P}} R_{\mathrm{L}}}{\sqrt{1+\left(2 \pi f_{\mathrm{PL}}\left(C_{\mathrm{P}}+C_{\mathrm{B}}\right) R_{\mathrm{L}}\right)^{2}}}
$$

En la Figura 2-16 se muestran curvas típicas de esta expresión, las cuales presentan dos asíntotas. Para bajos valores de $R_{\mathrm{L}} ; V_{\mathrm{L}}$ solo depende de $C_{\mathrm{P}}$ de acuerdo a:

Por otro lado para altos valores de $R_{\mathrm{L}}$ :

$$
\begin{gathered}
\left|V_{\mathrm{L}}\right| \stackrel{R_{\mathrm{L}} \rightarrow 0}{\longrightarrow} V_{\mathrm{P}} 2 \pi f_{\mathrm{PL}} C_{\mathrm{P}} R_{\mathrm{L}} \\
\left|V_{\mathrm{L}}\right| \stackrel{R_{\mathrm{L}} \rightarrow \infty}{\longrightarrow} V_{\mathrm{P}} \frac{1}{1+C_{\mathrm{B}} / C_{\mathrm{P}}}
\end{gathered}
$$

codo de la curva, donde se intersecan estas asíntotas se da para el valor de resistencia $R_{\mathrm{L}}=R_{\mathrm{LC}}$ dado por:

$$
R_{\mathrm{LC}} \approx \frac{1}{2 \pi f_{\mathrm{PL}} C_{\mathrm{B}}}
$$

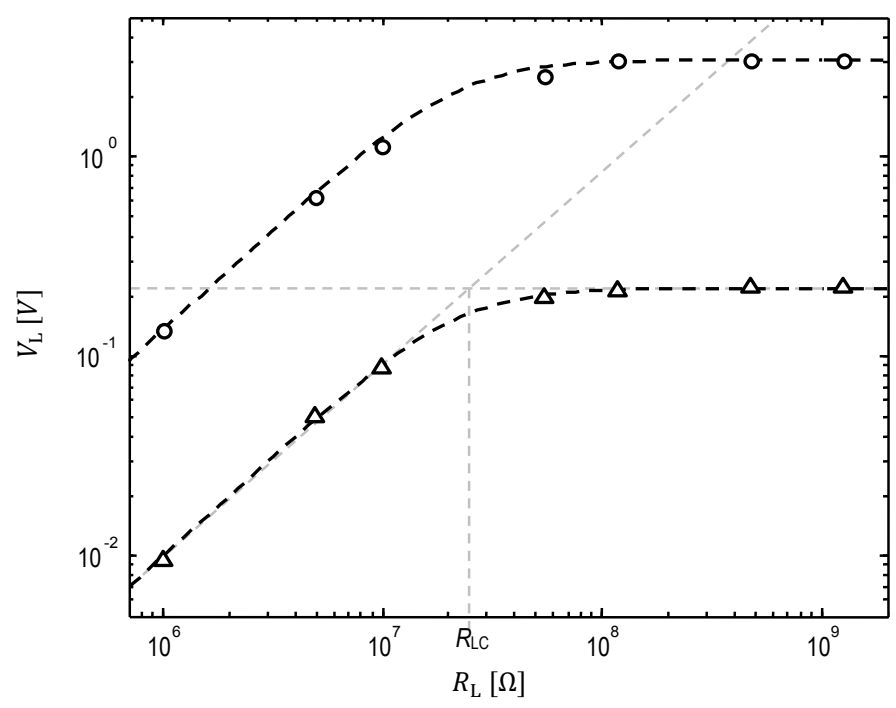

Figura 2-16: Medidas experimentales de $\mathbf{V}_{\mathbf{L}}$ para estimar $\mathbf{C}_{\mathbf{P}}$ y $\mathbf{C}_{\mathbf{B}}:(\boldsymbol{\Delta})$ sujeto parado cerca de un interruptor de luz (caso 4 de la Tabla 2-3), (o) sujeto parado y tocando el interruptor (caso 5). Las curvas negras corresponden a la ecuación (2-15) para los valores estimados de $\mathbf{C}_{\mathbf{P}}$ y $\mathbf{C}_{\mathbf{B}}$. Las líneas grises corresponden a las asíntotas del caso 4.

Es importante tener en cuenta que para estimar ambas capacidades $C_{\mathrm{P}}$ y $C_{\mathrm{B}}$ con buena sensibilidad, se deberán registrar medidas de $V_{\mathrm{L}}$ tanto por encima como por debajo de $R_{\mathrm{LC}}$. Si solo se toman medidas para $R_{\mathrm{L}}$ bajas, solo podrá ser estimada $C_{\mathrm{P}}$. Por otro lado, si las medidas de $V_{\mathrm{L}}$ corresponden a valores altos de $R_{\mathrm{L}}$, solo podrá 
estimarse la relación entre las capacidades $C_{\mathrm{P}} / C_{\mathrm{B}}$ pero no sus valores individuales. Considerando $C_{\mathrm{B}}=$ $100 p \mathrm{~F}$, resulta una $R_{\mathrm{LC}}$ de alrededor de $30 \mathrm{M} \Omega$ requiriendo que $R_{\mathrm{L}}$ adopte valores de algún $\mathrm{M} \Omega$ hasta cientos de $\mathrm{M} \Omega$.

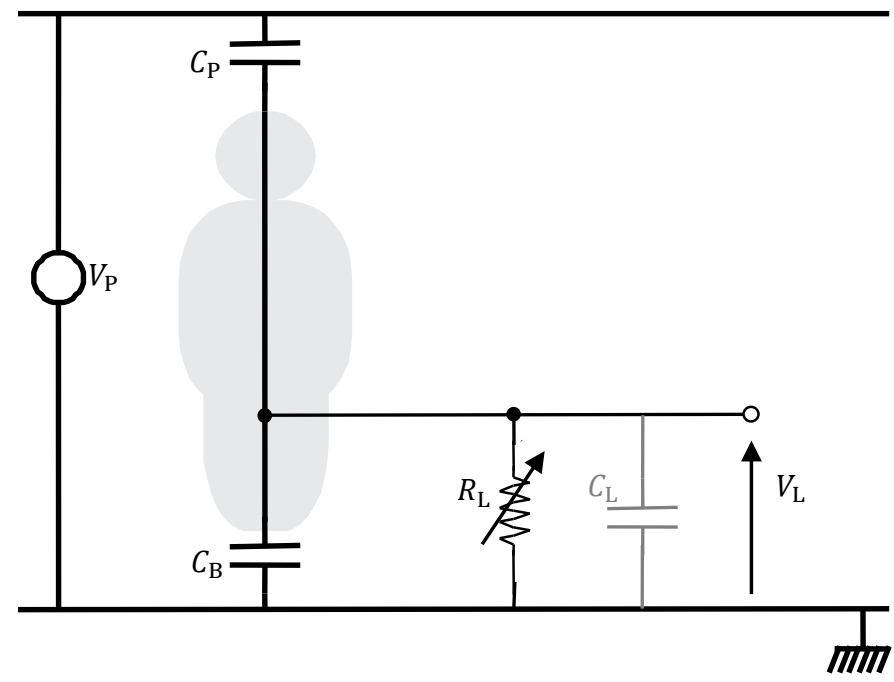

Figura 2-17: Circuito representativo del método de estimación de las capacidades de acoplamiento.

El mismo método puede aplicarse para estimar los valores de $C_{\mathrm{ISO}}$ y $C_{\mathrm{SUP}}$. Para esto hay que reemplazar al sujeto por el común del amplificador, pero teniendo en cuenta que, en amplificadores bien aislados, $C_{\text {ISo }}$ puede ser de apenas algunos picofaradios, se requerirán valores de $R_{\mathrm{L}}$ de algunos $\mathrm{G} \Omega$. Otro factor limitante son las capacidades parásitas en paralelo a $R_{\mathrm{L}}$ (designadas en la Figura 2-17 como una única capacidad concentrada $C_{\mathrm{L}}$ ). Estas se deben principalmente a las capacidades de los cables y limitan la mínima capacidad medible, aunque es posible desafectarlas de los cálculos.

\subsubsection{Circuito utilizado}

El circuito propuesto es una mejora del utilizado por Spinelli (2007), incorporando un circuito manejador de blindaje que compensa los efectos de la capacidad del cable blindado, permitiendo medir muy bajas capacidades, como las asociadas al $A B$, extendiendo así las posibilidades del método de medida. El diseño también incluye un filtro pasabanda, que selecciona los $50 \mathrm{~Hz}$ de la red, eliminando la componente de continua y la interferencia de alta frecuencia. De este modo es posible utilizar un simple voltímetro de "valor eficaz verdadero" para la medición de $V_{L}$.

El circuito completo de medida se muestra en la Figura 2-18. Mediante ocho llaves se seleccionan los diferentes valores de $R_{\mathrm{L}}$. Se utilizaron resistores simples $\left(R_{4}\right)$ para los cuatro valores más bajos de resistencia, entre $470 \mathrm{k} \Omega$ y $10 \mathrm{M} \Omega$, mientras que los cuatro valores más altos de $R_{\mathrm{L}}$ fueron simulados con una topología bootstrap. Asumiendo una impedancia de entrada y una ganancia de lazo abierto infinitas para el amplificador operacional, el valor de $R_{\mathrm{L}}$ equivalente resulta:

$$
R_{\mathrm{L}} \approx R_{1}+R_{2}+R_{1} \frac{R_{2}}{R_{3}}
$$

Utilizando $R_{1}=4,7 \mathrm{M} \Omega, R_{3}=100 \mathrm{k} \Omega$ y $R_{2}$ variando de $1 \mathrm{M} \Omega$ a $22 \mathrm{M} \Omega, R_{\mathrm{L}}$ varía aproximadamente de $50 \mathrm{M} \Omega$ a $1 \mathrm{G} \Omega$. 
En la Tabla 2-1 pueden verse los valores utilizados para $R_{2}$ y $R_{4}$, junto con el valor resultante de la $R_{\mathrm{L}}$ en cada caso.

\begin{tabular}{cccc|c}
\hline$R_{1}$ & $R_{2}$ & $R_{3}$ & $R_{4}$ & $R_{\mathrm{L}}$ \\
\hline $4,7 \mathrm{M} \Omega$ & $\infty$ & $100 \mathrm{k} \Omega$ & $470 \mathrm{k} \Omega$ & $470 \mathrm{k} \Omega$ \\
$4,7 \mathrm{M} \Omega$ & $\infty$ & $100 \mathrm{k} \Omega$ & $1 \mathrm{M} \Omega$ & $1 \mathrm{M} \Omega$ \\
$4,7 \mathrm{M} \Omega$ & $\infty$ & $100 \mathrm{k} \Omega$ & $4,7 \mathrm{M} \Omega$ & $4,7 \mathrm{M} \Omega$ \\
$4,7 \mathrm{M} \Omega$ & $\infty$ & $100 \mathrm{k} \Omega$ & $10 \mathrm{M} \Omega$ & $10 \mathrm{M} \Omega$ \\
$4,7 \mathrm{M} \Omega$ & $1 \mathrm{M} \Omega$ & $100 \mathrm{k} \Omega$ & $\infty$ & $53 \mathrm{M} \Omega$ \\
$4,7 \mathrm{M} \Omega$ & $2,2 \mathrm{M} \Omega$ & $100 \mathrm{k} \Omega$ & $\infty$ & $110 \mathrm{M} \Omega$ \\
$4,7 \mathrm{M} \Omega$ & $10 \mathrm{M} \Omega$ & $100 \mathrm{k} \Omega$ & $\infty$ & $485 \mathrm{M} \Omega$ \\
$4,7 \mathrm{M} \Omega$ & $22 \mathrm{M} \Omega$ & $100 \mathrm{k} \Omega$ & $\infty$ & $1,06 \mathrm{G} \Omega$ \\
\hline
\end{tabular}

Tabla 2-1: Valores utilizados de $\boldsymbol{R}_{\mathbf{2}}$ y $\boldsymbol{R}_{\mathbf{4}}$, en cada caso, y los respectivos valores de $R_{\mathrm{L}}$.

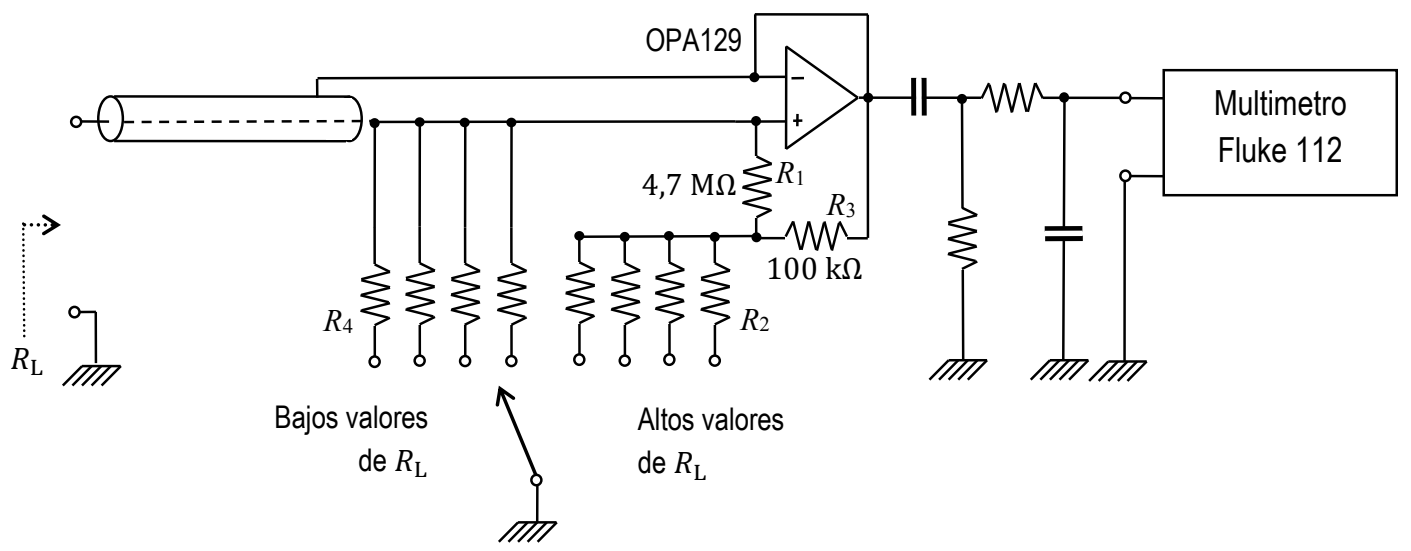

Figura 2-18: Circuito implementado para realizar las mediciones de $\boldsymbol{V}_{\mathbf{L}}$ y poder estimar las capacidades de acoplamiento.

Los valores de las capacidades parásitas se estimaron ajustando la curva teórica dada por (2-15) a los datos experimentales con el criterio del mínimo error cuadrático medio. Con este procedimiento se obtuvieron las capacidades asociadas al sujeto y al $A B$, para condiciones de uso típicas. Se realizaron diversas pruebas variando la posición del sujeto, las condiciones de EMI del entorno y los equipos biomédicos. 


\subsubsection{Resultados experimentales}

\subsubsection{Capacidades $C_{\text {ISo }}$ y $C_{\text {SUP }}$ asociadas al amplificador}

La técnica propuesta fue empleada para estimar las capacidades parásitas de algunos sistemas de adquisición de biopotenciales típicos, resultando los datos experimentales de la Figura 2-19, que son resumidos en la Tabla 2-2. Para un sistema de adquisición multicanal, alimentado desde la red de $220 \mathrm{~V} / 50 \mathrm{~Hz}$ a través de un transformador de aislación de grado médico, $C_{\mathrm{ISO}}$ resulta ser de aproximadamente $100 \mathrm{pF}$ y $C_{\mathrm{SUP}}$ de unos pocos picofaradios. Para dispositivos de pequeño tamaño, alimentados a batería, estas capacidades se reducen a 30 $\mathrm{pF}$ y centésimas de picofaradio respectivamente. Para estos valores tan bajos de capacidad, $C_{\mathrm{ISO}}$ depende del lugar donde el dispositivo es apoyado, dado que este objeto también estará vinculado tanto con tierra como con la línea de energía a través de sus propias capacidades de acoplamiento.

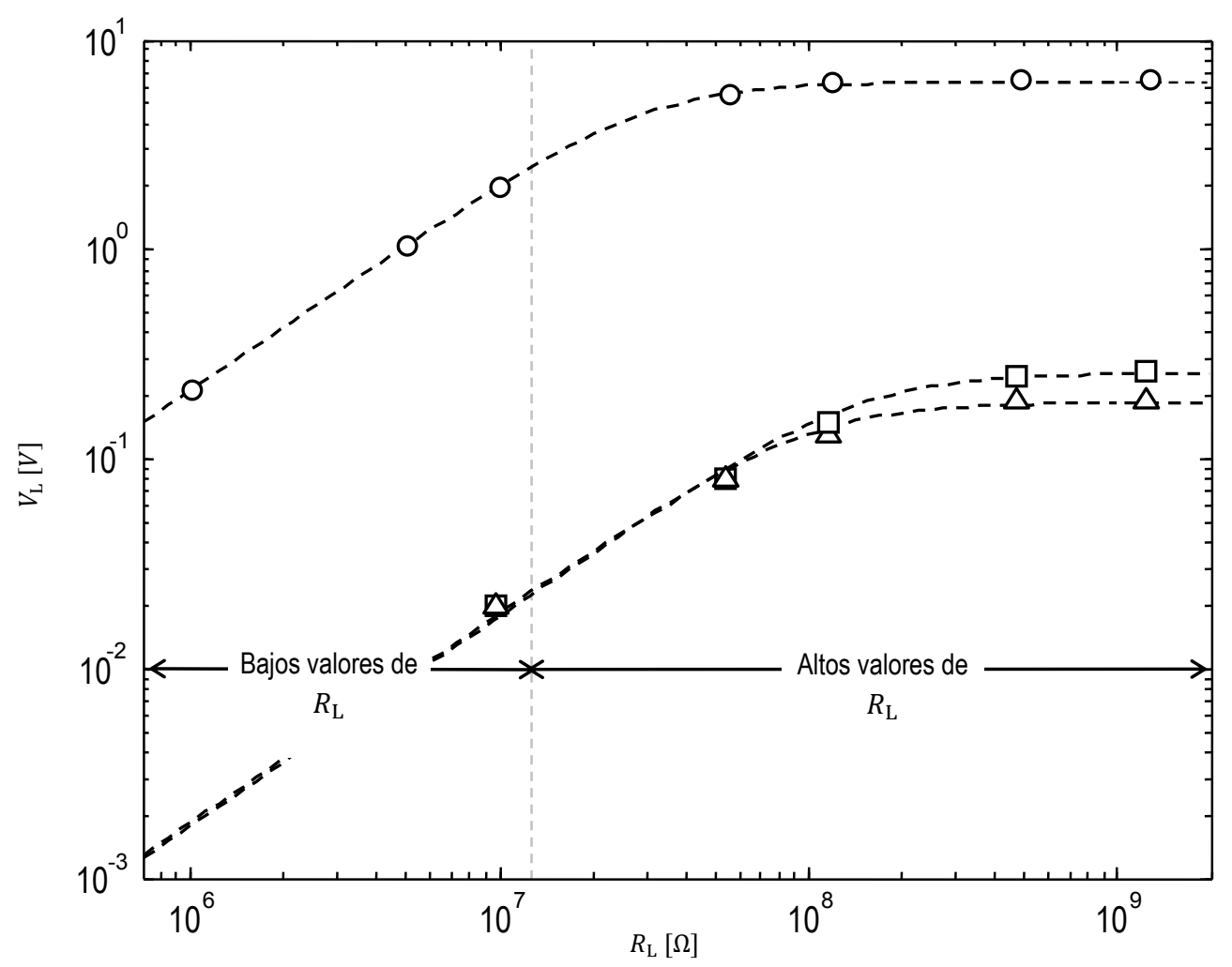

Figura 2-19: Medidas experimentales de $\boldsymbol{V}_{\mathrm{L}}$ para estimar $\boldsymbol{C}_{\mathrm{ISO}}$ y $\boldsymbol{C}_{\mathrm{SUP}}$ : (o) sistema multicanal alimentado de la red (caso 1 de la Tabla 2-2), () sistema USB aislado, alimentado a baterías (caso 2), ( $\Delta$ ) sistema inalámbrico, alimentado a baterías (caso 3). Las curvas corresponden a la ecuación (2-15) para los valores estimados de $\boldsymbol{C}_{\text {ISo }}$ y $\boldsymbol{C}_{\mathrm{SUP}}$. 


\begin{tabular}{llcc}
\hline$\#$ & Descripción del AB & $\boldsymbol{C}_{\text {ISo }}[\mathbf{p F}]$ & $\boldsymbol{C}_{\text {SUP }}[\mathbf{p F}]$ \\
\hline $\mathbf{1}$ & Sistema EEG multicanal alimentado de la red & 99.6 & 3.31 \\
$\mathbf{2}$ & $\begin{array}{l}\text { Sistema EMG con USB aislado, alimentado a } \\
\text { baterías }\end{array}$ & 29.2 & 0.03 \\
$\mathbf{3}$ & $\begin{array}{l}\text { Sistema EEG inalámbrico, alimentado a } \\
\text { baterías }\end{array}$ & 18.4 & 0.03 \\
\hline
\end{tabular}

Tabla 2-2: Capacidades $\boldsymbol{C}_{\text {ISo }}$ y $\boldsymbol{C}_{\text {SUP }}$ para distintos tipos de AB

\subsubsection{Capacidades $C_{\mathrm{B}}$ y $C_{\mathrm{P}}$ asociadas a la persona}

Las capacidades parásitas asociadas al sujeto fueron estimadas para distintas condiciones, resultando en los datos experimentales resumidos en la Tabla 2-3, que dieron origen a la publicación (Haberman et al 2011). En la Tabla 2-3 también se incluyen los valores esperados de $v_{\mathrm{CM}}$ (esto es, la tensión desarrollada sobre la impedancia $Z_{\mathrm{EG}}$ del tercer electrodo) para dos tipos de sistema de adquisición de biopotenciales: un sistema multicanal alimentado de la red (caso 1 de la Tabla 2-2) y un sistema pequeño alimentado a baterías (caso 2 de la Tabla 2-2). Para el cálculo de $v_{\mathrm{CM}}$, se asumió una impedancia de tercer electrodo resistiva, $Z_{\mathrm{EG}}=100 \mathrm{k} \Omega$, y se utilizó la ecuación (2-6).

Se observa que la capacidad $C_{\mathrm{B}}$ entre el sujeto y tierra, varía entre $100 \mathrm{pF}$ y $200 \mathrm{pF}$ dependiendo de su vinculación con la tierra (suela del calzado, pies en el suelo, pies levantados), pero el valor crece significativamente con el área efectiva del sujeto. De esta manera se incrementa a cerca de $300 \mathrm{pF}$ cuando éste toca un escritorio o cuando es tocado por otra persona, llegando a varios nanofaradios cuando la persona toca un objeto de gran tamaño como un armario. Respecto de la capacidad $C_{\mathrm{P}}$ entre sujeto y la línea de energía, esta es fuertemente dependiente de la cercanía a los cables de energía y a los equipos alimentados desde la línea. Puede ser tan baja como centésimas de picofaradios cuando el sujeto está en el medio de una habitación y llegar a algunos picofaradios cuando toca un interruptor de pared.

En las Figura 2-16 y Figura 2-19 se muestran algunos datos experimentales, los cuales muestran un muy buen ajuste con las curvas teóricas dadas por (2-15), validando de esta forma el modelo EMI simplificado de la Figura 2-12. 


\begin{tabular}{|c|c|c|c|c|c|}
\hline$\#$ & Condición del sujeto & $C_{\mathrm{B}}[\mathrm{pF}]$ & $C_{\mathrm{P}}[\mathrm{pF}]$ & $v_{\mathrm{CM}}[\mathrm{mV}](\mathrm{A})$ & $v_{\mathrm{CM}}[\mathrm{mV}](\mathrm{B})$ \\
\hline 1 & Parado en el medio de la habitación & 177 & 0.06 & 14.3 & 0.2 \\
\hline 2 & Parado cerca de una lámpara & 145 & 1.53 & 9.1 & 1.0 \\
\hline 3 & $\begin{array}{l}\text { Parado cerca de una lámpara y } \\
\text { tocando el interruptor }\end{array}$ & 145 & 2.80 & 5.5 & 2.0 \\
\hline 4 & Parado cerca de un interruptor de luz & 143 & 0.15 & 12.9 & 0.1 \\
\hline 5 & Parado y tocando un interruptor de luz & 136 & 1.76 & 7.9 & 1.2 \\
\hline 6 & Parado y tocando un armario metálico & 3932 & 1.90 & 22.0 & 0.2 \\
\hline 7 & $\begin{array}{l}\text { Sentado en el medio de la habitación } \\
\text { (pies en el suelo) }\end{array}$ & 190 & 0.07 & 14.7 & 0.2 \\
\hline 8 & $\begin{array}{l}\text { Sentado en el medio de la habitación } \\
\text { (pies levantados) }\end{array}$ & 116 & 0.07 & 11.9 & 0.1 \\
\hline 9 & $\begin{array}{l}\text { Sentado frente a una computadora } \\
\text { usando el mouse }\end{array}$ & 224 & 0.47 & 14.7 & 0.0 \\
\hline 10 & $\begin{array}{l}\text { Sentado (con otra persona parada } \\
\text { cerca) }\end{array}$ & 154 & 0.84 & 11.4 & 0.4 \\
\hline 11 & $\begin{array}{l}\text { Sentado (con otra persona parada } \\
\text { cerca y tocándolo) }\end{array}$ & 273 & 2.06 & 12.8 & 0.7 \\
\hline 12 & $\begin{array}{l}\text { Sentado (con otra persona parada } \\
\text { cerca y tocándolo. Con una lámpara } \\
\text { cerca ) }\end{array}$ & 224 & 1.87 & 11.7 & 0.8 \\
\hline 13 & $\begin{array}{l}\text { Sentado con una lámpara cerca y un } \\
\text { pie en el suelo }\end{array}$ & 121 & 2.06 & 6.1 & 1.7 \\
\hline 14 & $\begin{array}{l}\text { Sentado con una lámpara cerca y dos } \\
\text { pies en el suelo }\end{array}$ & 158 & 2.04 & 8.4 & 1.3 \\
\hline
\end{tabular}

Tabla 2-3: Capacidades $\boldsymbol{C}_{\mathbf{B}}$ y $\boldsymbol{C}_{\mathbf{P}}$ para distintas condiciones del sujeto y su entorno, y valor de $\boldsymbol{v}_{\mathrm{CM}}$ esperado para (A) un sistema multicanal alimentado de la red (caso 1 de la Tabla 2-2) y (B) un sistema pequeño alimentado a baterías (caso 2 de la Tabla 2-2).

\subsection{Conclusiones}

La instrumentación de biopotenciales requiere poder resolver diferencias de tensión muy pequeñas, del orden del microvolt, que deben captarse a través de impedancias de valor elevado y poco predecible. Por este motivo, el $A B$ deberá diseñarse teniendo en cuenta la posible introducción de interferencia electromagnética.

La principal fuente de EMI es la red de alimentación eléctrica, que introduce interferencia de $50 \mathrm{~Hz}$ en el $\mathrm{AB}$ acoplada mediante campos eléctricos o magnéticos. El estudio de los mecanismos de acoplamiento de EMI mediante modelos parciales permite identificar los factores predominantes en cada mecanismo de interferencia y 
posibilita la toma de acciones concretas en cada caso. Tal es el caso del uso de cable blindado o electrodos activos para mitigar la interferencia por corrientes de desplazamiento acopladas a los cables de electrodo. Esta última solución será utilizada en distintas implementaciones de DA en esta tesis.

En cuanto al CMRR, se evidencia que no son necesarios $A B$ con rechazos extremadamente grandes. Valores de CMRR mayores a $90 \mathrm{~dB}$ en el $\mathrm{AB}$ no proveen mejoras perceptibles frente al efecto divisor de potencial.

Para los diferentes mecanismos de interferencia, el nivel de EMI dependerá en gran medida del acoplamiento que exista con la fuente de interferencia, que para los mecanismos por acoplamiento eléctrico estará dado por las capacidades $C_{\mathrm{B}}, C_{\mathrm{P}}, C_{\mathrm{ISO}}$ y $C_{\mathrm{SuP}}$. Para predecir los valores de EMI resultantes que interferirán a los biopotenciales que deseamos medir, es usual la utilización de valores típicos. En vistas de poder realizar la medición de biopotenciales para controlar dispositivos de ayuda en ambientes domésticos, se estimaron las capacidades del modelo EMI para distintos contextos factibles en el uso cotidiano de un DA. 
Capítulo 2 - Biopotenciales e Interferencia Electromagnética 


\section{Capítulo 3}

\section{Electrodos para Dispositivos de Ayuda}

Este capítulo se focaliza sobre un componente que es fundamental en cualquier sistema de adquisición de señales biopotenciales: los electrodos. Estos reciben los biopotenciales y como tal son críticos para conservar la integridad de dichas señales antes de ser acondicionadas para su digitalización.

Los Dispositivos de Ayuda que estudiamos imponen requerimientos a los electrodos más estrictos que otras aplicaciones, como un estudio clínico de ECG, dado que deben utilizarse en sesiones más prolongadas y expuestos a condiciones de EMI más extremas.

El capítulo comienza describiendo los tipos de electrodos más utilizados, clasificados según el electrolito como húmedos, secos y capacitivos. Luego son presentadas diferentes implementaciones de electrodos activos (secos o húmedos) pensados para un Dispositivo de Ayuda controlado por EMG. Dichas implementaciones se fueron sucediendo a lo largo del desarrollo de la tesis, en un proceso que concluye en un electrodo activo para EMG con ganancia incorporada y muy alto CMRR. La presentación de este electrodo, que incorpora una reducción activa de la tensión de modo común, es acompañada por resultados experimentales.

Posteriormente se analiza la tecnología emergente de electrodos capacitivos, abordando su aspecto más crítico, que es su exceso de ruido. Se presenta una implementación de electrodos capacitivos desarrollados para adquirir señales de EMG y los resultados experimentales obtenidos. 


\subsection{Electrodos húmedos, secos y capacitivos}

Los electrodos son un componente importante en un sistema de adquisición de biopotenciales, ya que constituyen el primer punto donde pueden degradarse de manera irreversible las señales registradas.

Como fue mencionado previamente, desde el punto de vista eléctrico, los electrodos pueden ser modelados como una impedancia eléctrica en serie con una fuente de tensión continua de origen electroquímico, denominada tensión de offset del electrodo (ver Figura 3-1). Los electrodos introducen ruido aleatorio, cuyo valor es superior al que corresponde a la parte real (resistiva) de su impedancia de acuerdo a la ecuación de Nyquist (Huigen et al. 2002). Esto se expresa en la ecuación (3-1), donde $e_{Z_{\mathrm{E}}}^{2}(f)$ es la densidad espectral de potencia del ruido producido en el electrodo en $\mathrm{V}^{2} / \mathrm{Hz}, k=1,38 \cdot 10^{-23} \mathrm{~J} / \mathrm{K}$ es la constante de Boltzmann, $T$ es la temperatura absoluta en Kelvin y $\operatorname{Re}\left(Z_{\mathrm{E}}(f)\right)$ la parte real de la impedancia del electrodo.

$$
e_{Z_{\mathrm{E}}}^{2}(f)>\text { 4.k.T.Re }\left(Z_{\mathrm{E}}(f)\right)
$$

Los valores de los elementos del modelo de la Figura 3-1 dependen fuertemente del tipo de electrodo, área, forma, y especialmente del uso o no de electrolito entre el electrodo metálico y la piel. Cuando se agrega electrolito se denominan electrodos húmedos; y si el electrodo metálico simplemente se apoya sobre la piel del paciente se trata de electrodos secos. Existe una tercera posibilidad donde no hay contacto directo entre el electrodo metálico y la piel, sino a través de un film dieléctrico. Esta última tecnología, actualmente en desarrollo, da origen a los electrodos capacitivos.

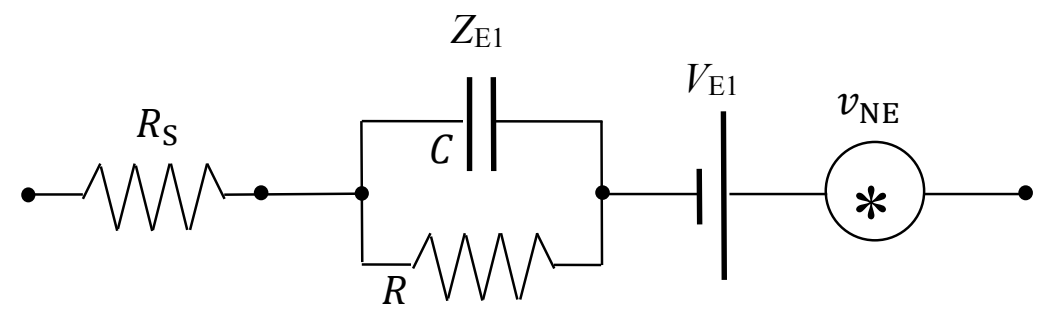

Figura 3-1: Modelo simplificado del electrodo que incluye la impedancia de electrodo, el potencial de continua $V_{\mathrm{E}}$ (offset) y el ruido propio del electrodo $v_{\mathrm{NE}}$.

\subsubsection{Electrodos húmedos}

Los electrodos más utilizados son los electrodos húmedos, los cuales hacen uso de geles o pastas electrolíticas entre la piel y la placa metálica del electrodo. En aplicaciones de ECG se cuenta con modelos comerciales autoadhesivos y descartables (ver Figura 3-2(a)). Estos electrodos también pueden utilizarse en algunas aplicaciones de EMG sobre músculos largos, donde los electrodos puedan ubicarse con una separación de algunos centímetros. En EEG, el pelo no permite utilizar estos electrodos y se utilizan otros que suelen tener forma de copa (ver Figura 3-2 (b)) o de disco metálicos con electrolito en pasta o gel que se coloca entre el electrodo metálico y el cuero cabelludo. Para facilitar el posicionamiento y la sujeción de los electrodos sobre la cabeza, suelen utilizarse gorras o vinchas. 
(a)

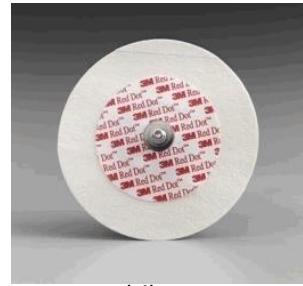

(d)

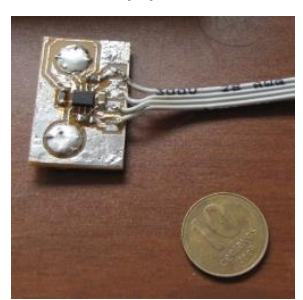

(b)

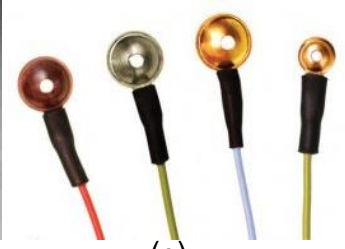

(e) (c)

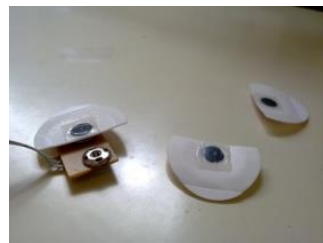

Figura 3-2: (a) Electrodo húmedo comercial para ECG (b) Electrodos de tipo "copa" para EEG (c-e) Pares de electrodos activos para EMG, con clips de acero inoxidable para acoplar a electrodos descartables (húmedos). Pueden utilizarse también como electrodos secos de acero inoxidable (aplicando directamente sobre la piel).

Los electrodos húmedos presentan características deseables, como baja impedancia y una rápida estabilización durante un período de uso acotado, pero presentan problemas en su uso prolongado (Searle y Kirkup 2000), que los hacen poco apropiados en DAs o Neuroprótesis:

- El gel se deshidrata, aumentando la impedancia del electrodo con el tiempo.

- Cuando se utilizan electrodos muy próximos entre sí (por ejemplo en arreglos bidimensionales para EMG), el gel puede fluir y "cortocircuitar" los electrodos.

- pueden generar irritación cuando son utilizados durante un período prolongado.

- El hecho de pegar y despegar los electrodos sobre la piel, o la colocación de pasta electrodo por electrodo, hacen de los procesos de colocación y extracción algo poco confortable para el usuario del DA y tedioso para quien lo asiste o coloca los electrodos.

- El soporte autoadhesivo va perdiendo fuerza y el electrodo termina separándose del sujeto.

Por estos aspectos, para la adquisición de biopotenciales en DAs son preferibles los electrodos secos, dado que dichos dispositivos deben ser prácticos (puesta en funcionamiento y desconexión rápidas, en pocos pasos) y robustos (funcionamiento estable ante el paso del tiempo y otras perturbaciones).

\subsubsection{Electrodos secos}

En esta clase de electrodos, la superficie metálica de los mismos está en contacto directo con la piel. Si bien no se agrega ninguna substancia electrolítica, el electrodo funciona utilizando la transpiración de la piel como electrolito. Esto implica que durante los primeros minutos luego de la colocación el electrodo presente una impedancia muy alta, acompañada por un alto exceso de ruido, que luego se estabilizarán en niveles comparables al de los electrodos húmedos (Huigen et al. 2002).

El modelo eléctrico de un electrodo seco no difiere demasiado del húmedo salvo por los valores de impedancia (y el consiguiente ruido) que suelen ser mayores y decrecen con el tiempo (Chi et al. 2010, Searle y Kirkup 2000).

Como se presentó en el Capítulo 2, las altas impedancias de electrodo (y los desbalances entre distintos electrodos) conducen a altos niveles de EMI. Dadas las altas impedancias iniciales de los electrodos secos, es 
necesario blindar el cable de conexión, o bien utilizar seguidores de tensión activos (buffers) en el electrodo mismo (electrodos activos).

\subsubsection{Valores del modelo de impedancia}

Si bien existen numerosos modelos para representar la impedancia de los electrodos, es común encontrar en la literatura la utilización del modelo simplificado representado en la Figura 3-1. En dicho modelo la resistencia $R_{\mathrm{S}}$ reproduce la conducción en los tejidos, mientras que el paralelo entre $R$ y $C$ modela principalmente el comportamiento en la conducción de biopotenciales del estrato córneo, la capa más externa de la piel.

Los valores posibles para dichos parámetros cubren un rango muy amplio y dependen de multiplicidad de factores, como el área del electrodo, el tipo de electrolito (en los electrodos húmedos), de la piel de la persona y de la fijación mecánica del electrodo. Es así que en la literatura se listan valores muy disimiles para los parámetros del modelo, tanto los valores teóricos como los experimentales. En la Tabla 3-1 se listan algunos de dichos valores, referenciándose en cada caso la fuente de donde se tomaron.

\begin{tabular}{|lcc|}
\hline \multicolumn{1}{|c}{ Referencia } & Electrodo húmedo & Electrodo seco \\
\hline (Valores teóricos) & - & $R=100 \ldots 600 \mathrm{k} \Omega \| C=60 \mathrm{nF} \ldots 2 \mu \mathrm{F}$ \\
Huigen et al., 2002 & $R_{\mathrm{S}}=500 \ldots 1000 \Omega$ \\
(Valores experimentales) & $10 \mathrm{k} \Omega \ldots 1 \mathrm{M} \Omega @ \mathrm{DC}$ & - \\
Chi et al., 2010 & $R 8901 \mathrm{M} \Omega \| C=10 \mathrm{nF}$ \\
(Valores teóricos) & $R=100 \mathrm{k} \Omega \| C=10 \mathrm{nF}$ & $R=1,3 \mathrm{M} \Omega \| C=12 \mathrm{nF}$ \\
(Valores experimentales) & $R=350 \mathrm{k} \Omega \| C=25 \mathrm{nF}$ & \\
Searle y Kirkup, 2000 & $|Z|=150 \mathrm{k} \Omega @ 57 \mathrm{~Hz}$ \\
(Valores experimentales) & $|Z|=300 \mathrm{k} \Omega @ 57 \mathrm{~Hz}$ \\
\hline
\end{tabular}

Tabla 3-1: Valores tomados de distintas fuentes bibliográficas para el modelo de impedancia de electrodo.

\subsubsection{Electrodos capacitivos}

Estos electrodos no utilizan electrolito y los potenciales se adquieren en forma capacitiva a través de una película dieléctrica o inclusive sobre la ropa, es decir no existe un contacto metal piel ni metal electrolito. El modelo de impedancia de estos electrodos no incluye tensión de offset, pero los valores de impedancia son muy elevados y las condiciones de medida más exigentes que en el caso de electrodos húmedos y secos. La utilización de este tipo de electrodos, que aún no tienen aplicación clínica, es relativamente reciente y sus características son 
significativamente distintas a la de los electrodos convencionales. Un análisis de esta técnica emergente se presenta en la sección 3.3 .

\subsection{Electrodos activos desarrollados para un DA controlado por EMG}

Remitiendo a lo expuesto en el Capítulo 2, la utilización de electrodos activos elimina el efecto de las corrientes de EMI acopladas a los cables de electrodos, e independiza la calidad de las señales adquiridas de las impedancias de los electrodos, del desapareamiento entre ellas, así como de la impedancia de entrada del $A B$ y de la longitud de los cables de electrodos. Otra ventaja es que con estos electrodos se hace innecesario el uso de cables mallados, pudiendo utilizarse cables más flexibles. Una desventaja es la necesidad de llevar la tensión de alimentación para los electrodos con cables adicionales.

Estos electrodos se diseñaron específicamente para la adquisición de EMG, siguiendo el objetivo de realizar una interface del tipo ON/OFF controlada por la contracción de distintos músculos. En particular, se hace hincapié en la adquisición de EMG del músculo facial cigomático mayor, dado un caso particular de aplicación del DA, pero los electrodos pueden utilizarse con diversos músculos del cuerpo. También se presentan ensayos realizados sobre el antebrazo.

Las señales de EMG son particularmente apropiadas para utilizar electrodos activos, pues se registran como diferencias de potencial entre pares de electrodos próximos. Esto permite implementar electrodos activos de a pares, compartiendo la tensión de alimentación y un mismo soporte mecánico (Figura 3-2 (c-f)), que fija la distancia inter-electrodo y a su vez sirve para contener la electrónica necesaria. Así, se rechaza la EMI por corrientes de desplazamiento en los cables de medida, quedando como fuentes principales de EMI la tensión de modo común y el campo magnético externo. Para reducir esta última, se utiliza preferentemente cable plano, minimizando el área del lazo formado por los cables. En cuanto a la reducción de la EMI por modo común, esta se apoya en la utilización de la realimentación negativa de modo común mediante un tercer electrodo, lo cual se tratará en el Capítulo 4.

\subsubsection{Electrodos activos de ganancia unitaria}

En el proceso de desarrollo de un DA basado en señales de EMG de músculos faciales se investigaron diversas maneras para implementar dichos electrodos, resultando en la construcción de numerosos prototipos (Figura 3-2 $(c-f))$.

El diseño más simple, que consiste en dos seguidores de tensión (buffers) con ganancia unitaria (ver Figura 3-2 (d) y (f)), se desarrolló para poder utilizar electrodos secos, luego de ver los inconvenientes que acarreaba el uso de electrodos húmedos en dispositivos que requieren ser instalados y removidos frecuentemente y además operar durante períodos prolongados. Se implementaron diversos electrodos dobles, siguiendo el esquema de la Figura 3-3. Dichos electrodos se vinculan al $A B$ mediante cable plano de cuatro conductores, dos para la fuente simple de los operacionales y dos para la señal acondicionada que va del electrodo al $A B$. Para establecer el contacto seco con la piel del usuario se utilizaron broches de acero inoxidable de $10 \mathrm{~mm}$ de diámetro (ver Figura 3-2 (c)), separados $20 \mathrm{~mm}$ entre sus centros. Estos son compatibles con los electrodos descartables comerciales, de modo que si se lo desea se pueden utilizar electrodos húmedos, aprovechando las ventajas del electrodo activo en cuanto al rechazo de EMI. 


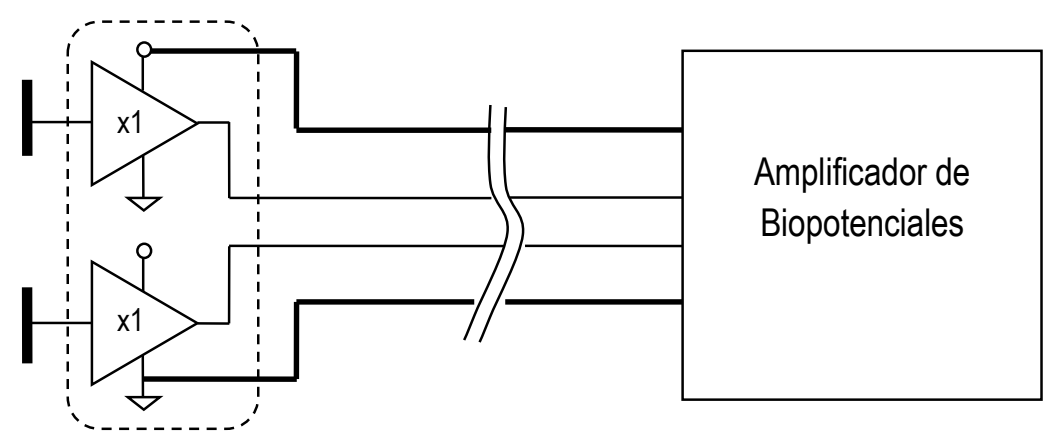

Figura 3-3: Electrodo activo doble (de ganancia unitaria) para medición de EMG

Los operacionales utilizados en los electrodos activos son del tipo CMOS, con muy bajas corrientes de ruido y de polarización, y tensión de ruido comparable al ruido de la interfaz electrodo-piel. En este caso se utilizó el TLC2272/4 en su versión de montaje superficial.

\subsubsection{Electrodos activos con ganancia}

Las tendencias en la adquisición de señales biopotenciales (Haberman et al. 2009) se orientan a la utilización de ADCs de tecnología $\Sigma-\Delta$ con más de 20 bits resolución efectiva. Dada estas altas resoluciones, dichos ADCs poseen entradas analógicas diferenciales, para rechazar diferencias de potencial en la masa analógica. Además, la tecnología $\Sigma-\Delta$ se basa en un sobremuestreo de la señal analógica ${ }^{2}$, por lo que se relajan los requerimientos para el diseño de una etapa de filtrado antialiasing. Estos factores se conjugan haciendo que la etapa de acondicionamiento analógico se pueda reducir a una simple etapa diferencial de dos operacionales, con una ganancia acotada del orden de los $10 \mathrm{~V} / \mathrm{V}$. Dada la baja ganancia es posible acoplar el amplificador en DC sin riesgo de saturación por el offset de electrodo. Las exigencias respecto al filtrado antialiasing son satisfechas por un filtro pasivo de primer orden.

Observando el esquema de la Figura 3-4(a) queda en evidencia que las características de alta impedancia de entrada y baja impedancia de salida que se logran con el buffer de ganancia unitaria, también se logran con la etapa completamente diferencial del AB. Por lo tanto, se decidió correr esta etapa directamente sobre el electrodo, es decir, el AB está directamente sobre el electrodo dejando solo la etapa de filtrado junto al ADC (ver Figura 3-4(b)). Un electrodo desarrollado que responde a dicho esquema se observa en la Figura 3-2(e).

\footnotetext{
${ }^{2}$ Esto significa que la señal es digitalizada a una tasa $f_{\mathrm{Si}}$ mayor a la tasa de entrega de datos $f_{\mathrm{So}}$. La relación entre ambas puede ser de 1000:1.
} 

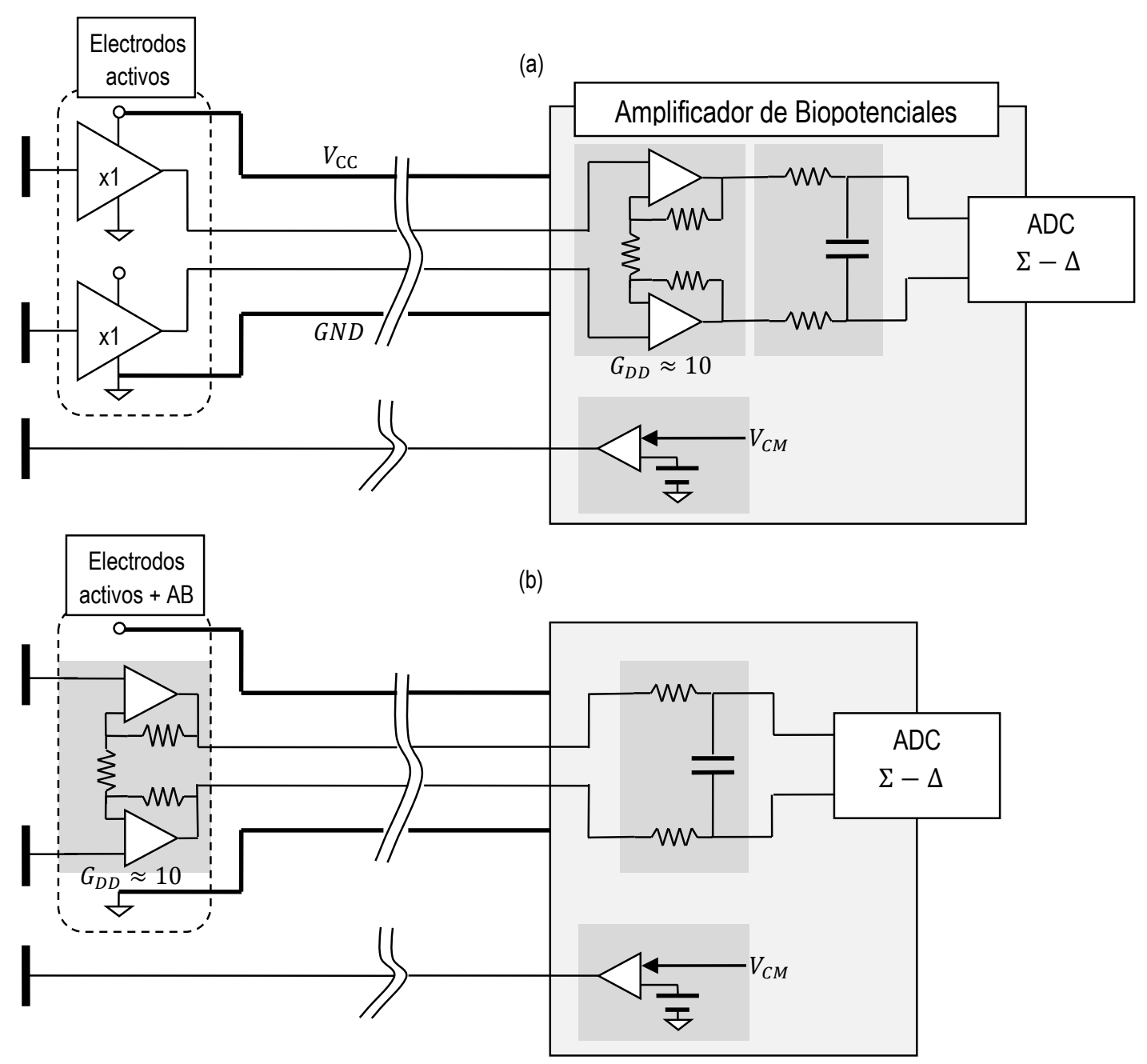

Figura 3-4: Detalle de las etapas analógicas cuando se utiliza un $\operatorname{ADC} \Sigma-\Delta$ de muy alta resolución. (a): Electrodo activo doble con ganancia unitaria y etapa de amplificación diferencial antes del ADC. (b): Electrodo activo que incluye la etapa de amplificación diferencial. La etapa analógica en la entrada del ADC se reduce a un filtro pasabajos diferencial de primer orden.

La topología del amplificador utilizado en el electrodo permite la amplificación de la señal diferencial de EMG con un muy alto CMRR, lo cual es posible gracias a la proximidad entre electrodos para la adquisición de señales de EMG. En casos donde los electrodos no se encuentren próximos entre sí, o bien se requiera la adquisición de múltiples canales, esta topología se vuelve difícil de implementar. En su lugar, puede amplificarse la señal biopotencial en el electrodo pero con una topología single ended (Deguen y Jäckel 2004), es decir medir el potencial de cada electrodo respecto de masa, conduciendo a valores muy pobres de CMRR. En estos casos se deberá acompañar dicha solución con algún mecanismo que asegure una importante reducción del modo común (Haberman y Spinelli 2012) como las que se tratan en el Capítulo 4.

\subsubsection{Electrodos activos con ganancia y realimentación de modo común}


En las secciones previas no se mencionó el "tercer electrodo" ni cómo establecer una tensión de modo común que optimice el rango de entrada de los amplificadores tanto como del ADC. Sin embargo, es necesario referir al usuario a un potencial de modo común compatible con el rango de entrada del $A B$. En el caso de los circuitos desarrollados este es un potencial continuo y se ubica en la mitad del rango de entrada. Considerando que los operacionales y el $\mathrm{ADC}$ son alimentados con fuente simple de $5 \mathrm{~V}$ y ambos admiten de $0 \mathrm{~V}$ a $5 \mathrm{~V}$ de modo común, la referencia de modo común se fijó en 2,5V.

Como se verá en el Capítulo 4, esta tensión continua no se aplica directamente al usuario, sino a través de un lazo de control que la toma como referencia. El circuito que implementa dicho lazo adopta el nombre de DRL (Driven Right Leg) de su uso en electrocardiografía, y en una primera versión fue implementado como un circuito aparte en la placa principal del equipo, requiriendo cableado adicional para el electrodo de realimentación. A fin de que el DA sea más práctico y genere menos complicaciones al usuario, en un diseño posterior se trasladó el DRL a la placa del electrodo, manteniendo los dos cables de alimentación y dos de la señal diferencial amplificada. De esta manera, toda la electrónica de acondicionamiento analógico queda en la placa de los electrodos (ver Figura 3-5), vinculada al resto del sistema por cuatro cables, que no necesitan blindajes. En este caso particular la distancia entre electrodos de medida es de $25 \mathrm{~mm}$.

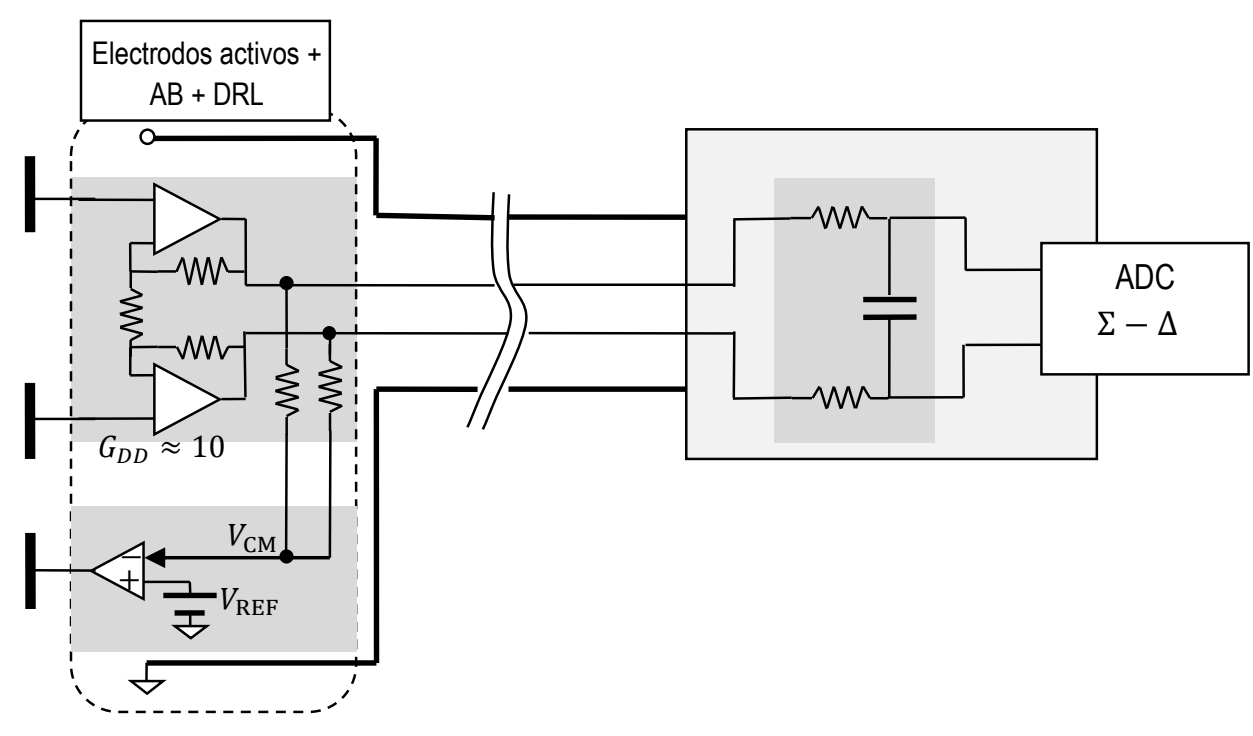

Figura 3-5: Diagrama conceptual del electrodo activo desarrollado, que incluye prácticamente todas las etapas analógicas que intervienen en la instrumentación de EMG.

\subsubsection{Evaluación de los electrodos activos construidos}

Los electrodos fueron evaluados, en primer lugar para determinar su nivel de ruido y luego operando en la adquisición de señales reales de EMG.

Ruido de la electrónica ( $A B$ y $A D C)$

En primera instancia se realizaron medidas del ruido propio de la electrónica, cortocircuitando los electrodos (ver Figura 3-6(a)) el resultado de dicha medida, evaluando 10 minutos de la señal digitalizada a 1200 sps (samples 
per second), fue un valor eficaz de ruido de $0.75 \mu \mathrm{V}_{\mathrm{RMS}}$ en el ancho de banda de $1 \mathrm{~Hz}$ a $380 \mathrm{~Hz}^{3}$. Considerando un ancho de banda de $30 \mathrm{~Hz}$ a $250 \mathrm{~Hz}$, que es el que se utiliza para la implementación de DA, dicho ruido se reduce a $0.56 \mu \mathrm{V}_{\mathrm{RMS}}$.

(a)

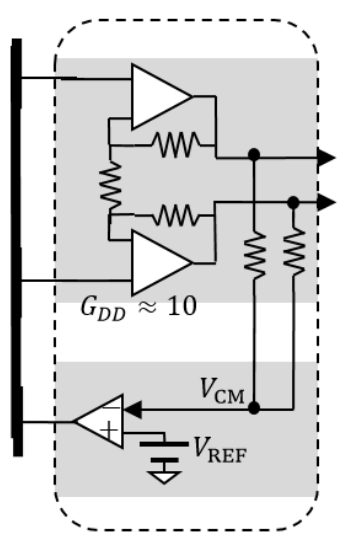

(b)

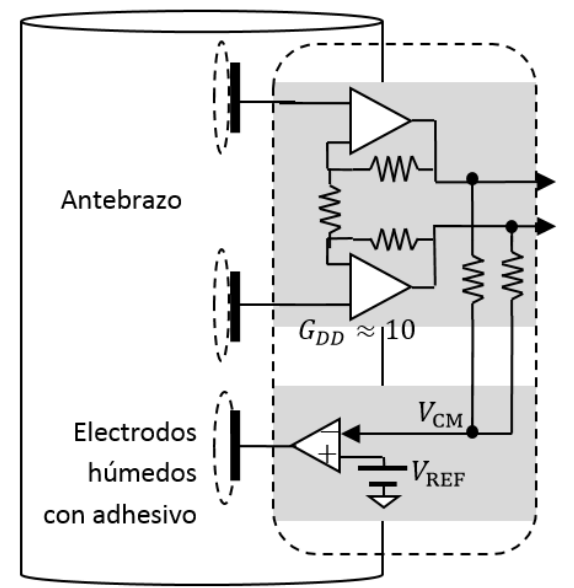

(c)

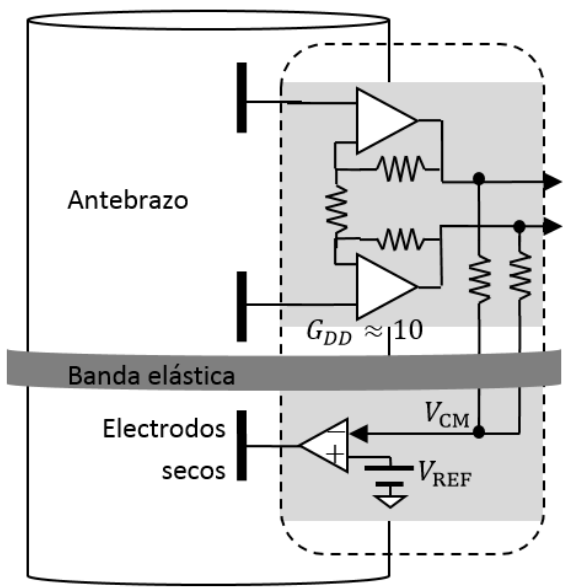

Figura 3-6: Esquema de las medidas realizadas. (a): Medida del ruido propio de la electrónica. Se cortocicuitaron todos los electrodos. (b): Medida del ruido del sistema con electrodos húmedos. (c): Medida del ruido del sistema con electrodos secos.

Ruido de la interfaz electrodo-piel

Se realizaron medidas del ruido aportado por la interfaz electrodo-piel. Para esto se colocó el electrodo desarrollado sobre el antebrazo de un voluntario (ver Figura 3-6 (b-c)). Durante las medidas, el sujeto mantuvo el antebrazo relajado para no generar señales de EMG. Se realizaron dos tandas de medidas, una para electrodos húmedos (descartables y autoadhesivos, marca $3 \mathrm{M}$ ) acoplados a la placa y otra para los electrodos metálicos directamente apoyados sobre la piel.

El resultado para los electrodos húmedos una tensión de ruido de $1.63 \mu \mathrm{V}_{\mathrm{RMS}}$ en el ancho de banda de $1 \mathrm{~Hz}$ a $380 \mathrm{~Hz}$, mientras que en el ancho de banda de $30 \mathrm{~Hz}$ a $250 \mathrm{~Hz}$, se reduce a $0.64 \mu \mathrm{V}_{\mathrm{RMS}}$.

Por otra parte, los electrodos secos, luego de unos pocos minutos alcanzaron niveles de ruido muy similares: $1.93 \mu \mathrm{V}_{\mathrm{RMS}}$ de $1 \mathrm{~Hz}$ a $380 \mathrm{~Hz}$ y $0.75 \mu \mathrm{V}_{\mathrm{RMS}}$ de $30 \mathrm{~Hz}$ a $250 \mathrm{~Hz}$.

\section{Señales de EMG adquiridas}

Con los electrodos secos colocados sobre el antebrazo, se realizaron contracciones isométricas de diferentes intensidades, a fin de comparar los niveles de amplitud y la distribución espectral de energía. Ya en el nivel más bajo de contracción, se observa que la señal supera ampliamente los niveles de ruido, con lo cual se demuestra la aptitud de la placa desarrollada para la adquisición de biopotenciales de EMG, apreciándose una muy buena relación señal a ruido, inclusive para la señal más débil de EMG que muestra amplitudes de decenas a centenas de $\mu \mathrm{V}$. Esto se observa en la Figura 3-7 y Figura 3-8 tanto para la distribución espectral de energía como para

\footnotetext{
${ }^{3}$ El ADC utilizado (un ADS1259) tiene la frecuencia de corte de $-3 \mathrm{~dB}$ en $380 \mathrm{~Hz}$, para la frecuencia de muestreo configurada.
} 
las señales en el dominio del tiempo, de lo cual se desprende que un diseño basado en electrodos secos activos permite adquirir señales de EMG con una calidad similar a la del caso de utilizar electrodos húmedos, superando con esto los problemas que devienen del uso prolongado de estos últimos.

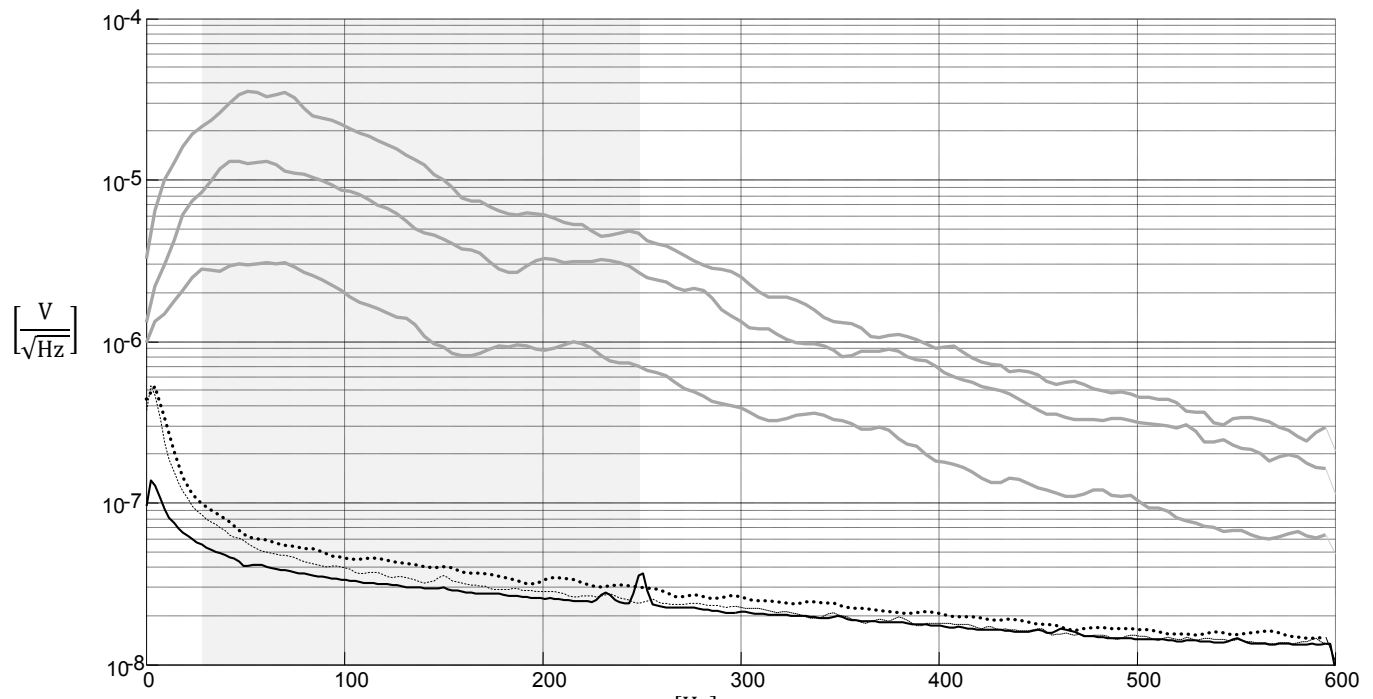

$[\mathrm{Hz}]$

Figura 3-7: Densidad espectral potencia en $[\mathrm{V} / \sqrt{\mathrm{Hz}}]$ de señales de EMG del antebrazo de distinta intensidad (en líneas grises) y de las diferentes fuentes de ruido: ruido del $A B$ con las entradas cortocircuitadas (en línea sólida negra), ruido del sistema con electrodos húmedos sobre la piel (línea a trazos negra) y ruido del sistema con electrodos secos sobre la piel (línea punteada negra). El área gris indica el ancho de banda utilizado para el procesamiento de la señal.

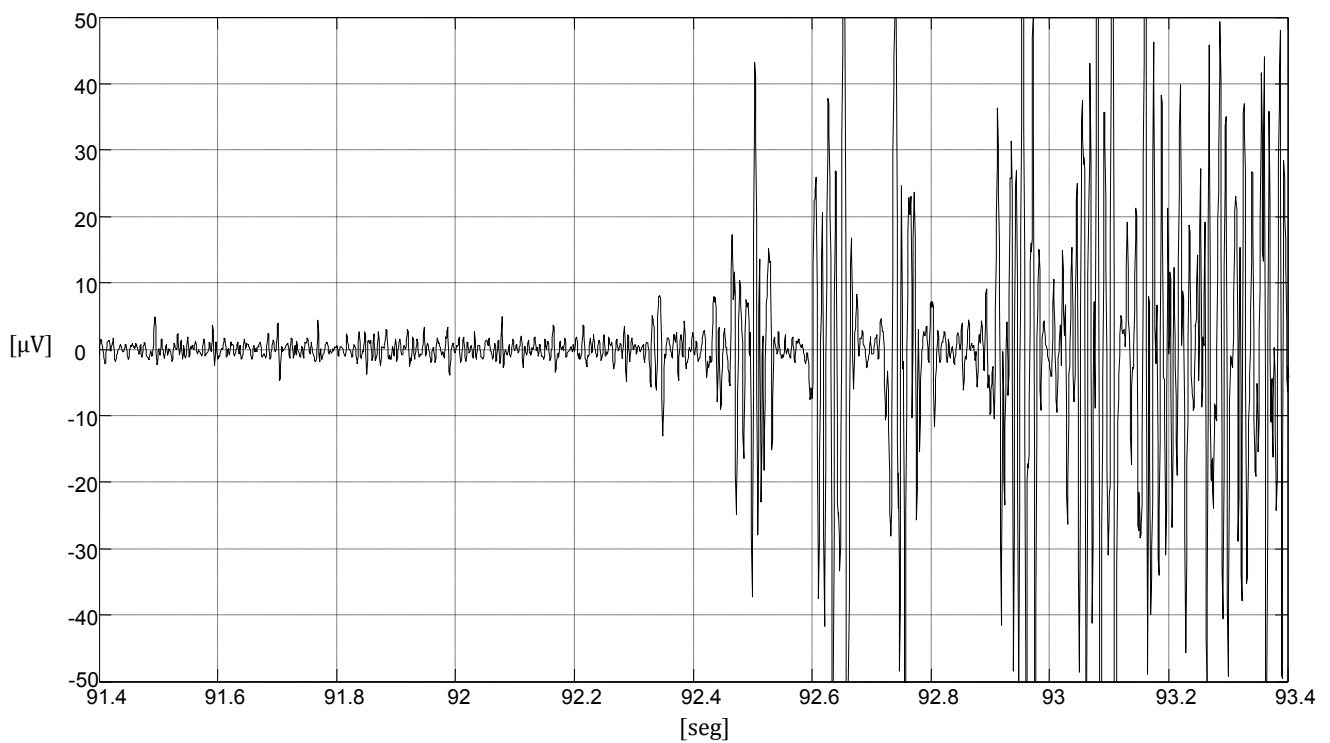

Figura 3-8: Segmento de 2 segundos de señal, adquirida con los electrodos secos sobre el antebrazo. Durante el primer segundo el músculo permanece en reposo (aunque se percibe actividad de EMG de muy baja amplitud) y luego se contrae levemente. La señal cruda ha sido pasada por un filtro pasabanda entre $30 \mathrm{~Hz}$ y $250 \mathrm{~Hz}$. 


\subsection{Electrodos Capacitivos}

Una alternativa relativamente reciente a los electrodos húmedos y secos son los electrodos capacitivos. Esta clase de electrodos puede medir los biopotenciales a través de un material dieléctrico, sin que un electrolito o metal entre en contacto directo con la piel, haciendo posible la medición de biopotenciales con sensores embebidos en la vestimenta o incluso sillas y camas (Ueno et al. 2007, Walter et al. 2011, Kim et al. 2005).

La idea de un electrodo capacitivo es simple, como se observa en la Figura 3-9. Las señales se acoplan a través de una capacidad, a un seguidor de tensión o buffer que adapta la impedancia mostrada por el electrodo al $A B$ (al igual que en los electrodos activos vistos anteriormente). Pero en este caso, dado que el buffer requiere un camino para sus corrientes de polarización, es necesaria la utilización del resistor $R_{\mathrm{Bias}}$ en la entrada.

Los primeros trabajos sobre esta tecnología datan de la década del 60 (Richardson 1967), con electrodos recubiertos por una fina capa aislante de óxido de aluminio. De esta manera se formaba entre el sujeto y la placa metálica del electrodo una capacidad de varios $\mathrm{nF}$ cuando el mismo era apoyado sobre la piel.

El desarrollo actual de los amplificadores operacionales permite medir biopotenciales a través de capacidades de acoplamiento de unos pocos pF, como sucede al medir a través de una tela de algodón. A pesar de la sencillez conceptual del circuito de la Figura 3-10, estos niveles de capacidad complican la implementación, ya que para obtener biopotenciales de ECG o EEG con frecuencias de corte de centésimas o décimas de $\mathrm{Hz}$ se requieren resistencias de polarización $R_{\text {Bias }}$ del orden del T $\Omega$ y capacidades de entrada por debajo del pF. A su vez, los altísimos valores de $R_{\text {Bias }}$ demandan muy bajas corrientes de polarización en los amplificadores y, como se verá, muy bajo ruido en corriente.

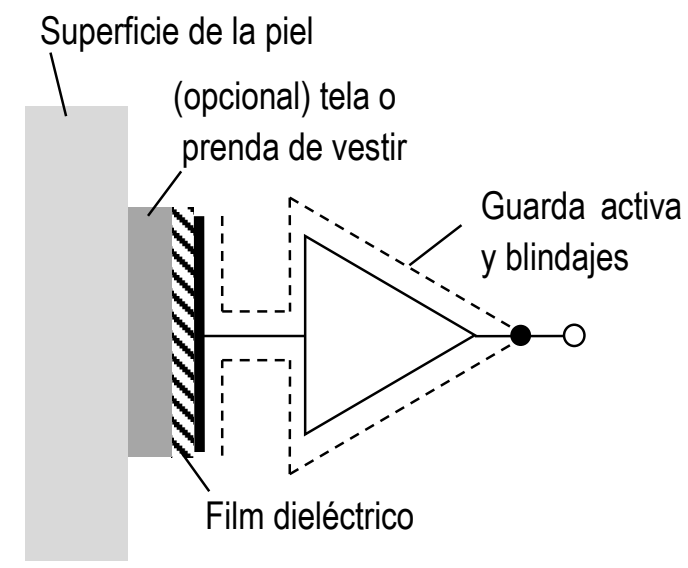

Figura 3-9: Esquema elemental de un electrodo capacitivo, el mismo consta de una placa metálica recubierta por un film dieléctrico y conectada a un buffer de muy alta impedancia de entrada. Se ilustra una posible aplicación donde se miden biopotenciales a través de una tela de algodón.

\subsubsection{Circuitos para electrodos capacitivos}

El circuito básico de un electrodos capacitivo consiste en un seguidor de tensión acoplado en alterna como se muestra en el circuito simplificado de la Figura 3-10. La principal dificultad es tratar con capacidades de acoplamiento $C_{\mathrm{s}}$ muy bajas, que requieren impedancias de entrada extra altas. Además, se debe proveer un 
camino para las corrientes de polarización del amplificador $i_{\text {Bias }}$, forzando a la inclusión del resistor $R_{\text {Bias }}$. Los altos valores de resistencia para $R_{\text {Bias }}$ introducen una mayor cantidad de ruido térmico que no logra ser "cortocircuitado" a través de $C_{\mathrm{s}}$, como ocurriría en una aplicación estándar.

La impedancia de entrada, formada por $R_{\mathrm{Bias}}$ en paralelo $\operatorname{con} C_{\mathrm{in}}$, debe ser mucho más grande que la impedancia de la fuentes impuesta por $C_{\mathrm{S}}$, para evitar la distorsión y atenuación de la señal, y principalmente para lograr una ganancia independiente de la capacidad de acoplamiento $C_{\mathrm{S}}$. Dado el divisor de tensión capacitivo que forman $C_{\mathrm{S}}$ y $C_{\text {in }}$, será necesario un valor muy bajo para la capacidad de entrada $C_{\mathrm{in}}$. De lo contrario, debido al efecto divisor de potencial (Metting van rijn et al. 1990), se obtendrá un muy pobre rechazo de interferencias y artefactos de movimiento.

Frente a los requerimientos extremos que surgen al adquirir señales de ECG, debido a las bajas frecuencias involucradas (Spinelli y Haberman 2010), que implican valores de $R_{\text {Bias }}$ del orden del T $\Omega$ para valores de $C_{\mathrm{S}}$ de decenas de pF; la señal de EMG se ve mucho más apropiada para este tipo de electrodos con contenido en frecuencia por encima de los $10 \mathrm{~Hz}$, lo cual relaja los valores de $R_{\text {Bias }}$ requeridos a algunos $\mathrm{G} \Omega$.

(a)

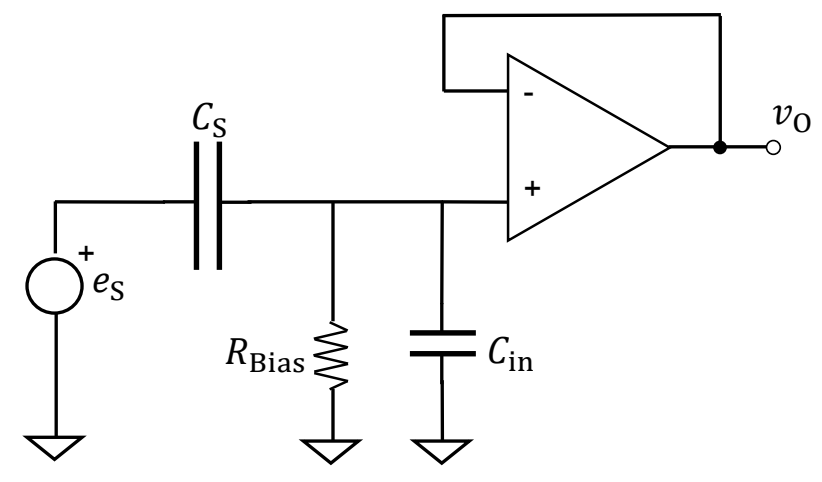

(b)

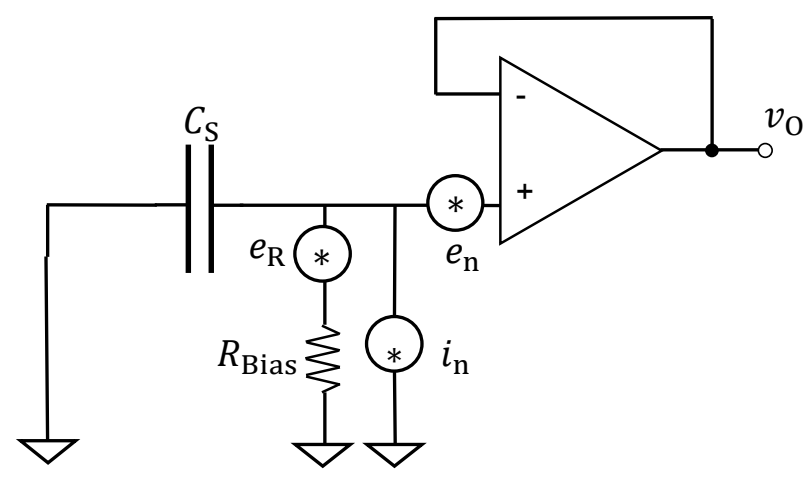

Figura 3-10: Circuito simplificado del electrodo capacitivo. (a) circuito utilizado en el análisis de la transferencia de la señal. (b) circuito utilizado en el análisis de ruido.

\subsubsection{Utilización de guardas activas}

Las altas impedancias asociadas a la entrada del buffer hacen que dicho nodo del circuito sea muy susceptible a cualquier corriente parásita. Por lo tanto, estas corrientes suelen limitarse rodeando cualquier pista o plano correspondiente a dicho nodo mediante la salida del buffer, que idealmente es la misma señal que la entrada pero con baja impedancia (ver Figura 3-11). De esta forma, al estar al "mismo" potencial se hacen despreciables las corrientes parásitas a la vez que se blinda al nodo de entrada frente a otro tipo de interferencias. 


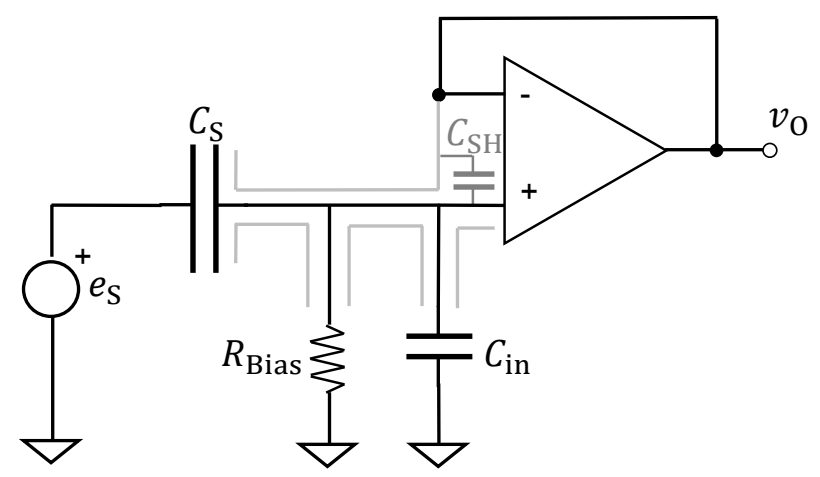

Figura 3-11: Circuito simplificado del electrodo capacitivo que incluye la guarda utilizada (en color gris) para proteger al nodo de entrada de posibles perturbaciones. Nótese la aparición de una capacidad parásita (modelada como $C_{\mathrm{SH}}$ ) entre el nodo de entrada y la guarda.

\subsubsection{Análisis del exceso de ruido}

Cuando se utilizan electrodos húmedos o secos, el nivel de ruido, dado su origen electroquímico, excede al previsto por la ecuación de Nyquist considerando la parte resistiva de la impedancia de electrodo (Huigen et al. 2002). No obstante, a pesar de no poder estimarlo fehacientemente, estos niveles están por debajo que los niveles de las señales de EMG y son comparables al ruido de la electrónica. En dichos casos, respecto al ruido de la electrónica, solo es de interés el ruido en tensión del operacional utilizado como buffer, dado que la relativa baja impedancia de electrodo (menor al $\mathrm{M} \Omega$ en prácticamente todo el espectro de interés) no convierte en significativas las bajas corrientes de ruido de los amplificadores con entrada FET o MOS, menores al fA $/ \sqrt{\mathrm{Hz}}$. En un electrodo capacitivo, gran parte del ruido total se produce en la propia electrónica del electrodo y será dependiente de la capacidad de acoplamiento $C_{\mathrm{S}}$. El ruido del resistor $R_{\text {Bias }}$ que es de muy alto valor impacta en el ruido total a la salida del electrodo $e_{\mathrm{O}}$ de dos maneras: por su ruido térmico $e_{\mathrm{R}}$ y amplificando el ruido en corriente $i_{\mathrm{n}}$ del amplificador operacional (Spinelli y Haberman 2010):

$$
e_{\mathrm{O}}^{2}(f)=e_{\mathrm{R}}^{2} \frac{1}{1+\left(2 \pi R_{\text {Bias }} C_{\mathrm{S}} f\right)^{2}}+i_{\mathrm{n}}^{2} \frac{R_{\text {Bias }}^{2}}{1+\left(2 \pi R_{\text {Bias }} C_{\mathrm{S}} f\right)^{2}}+e_{\mathrm{n}}^{2}
$$

Definiendo la frecuencia de corte inferior de $-3 \mathrm{~dB}$ como $f_{\mathrm{N}}$ :

$$
f_{\mathrm{N}}=\frac{1}{2 \pi R_{\text {Bias }} C_{\mathrm{S}}}
$$

La ecuación (3-2) se reduce a: 


$$
e_{\mathrm{O}}^{2}(f)=e_{\mathrm{R}}^{2} \frac{1}{1+\left(\frac{f}{f_{\mathrm{N}}}\right)^{2}}+i_{\mathrm{n}}^{2} \frac{R_{\text {Bias }}^{2}}{1+\left(\frac{f}{f_{\mathrm{N}}}\right)^{2}}+e_{\mathrm{n}}^{2}
$$

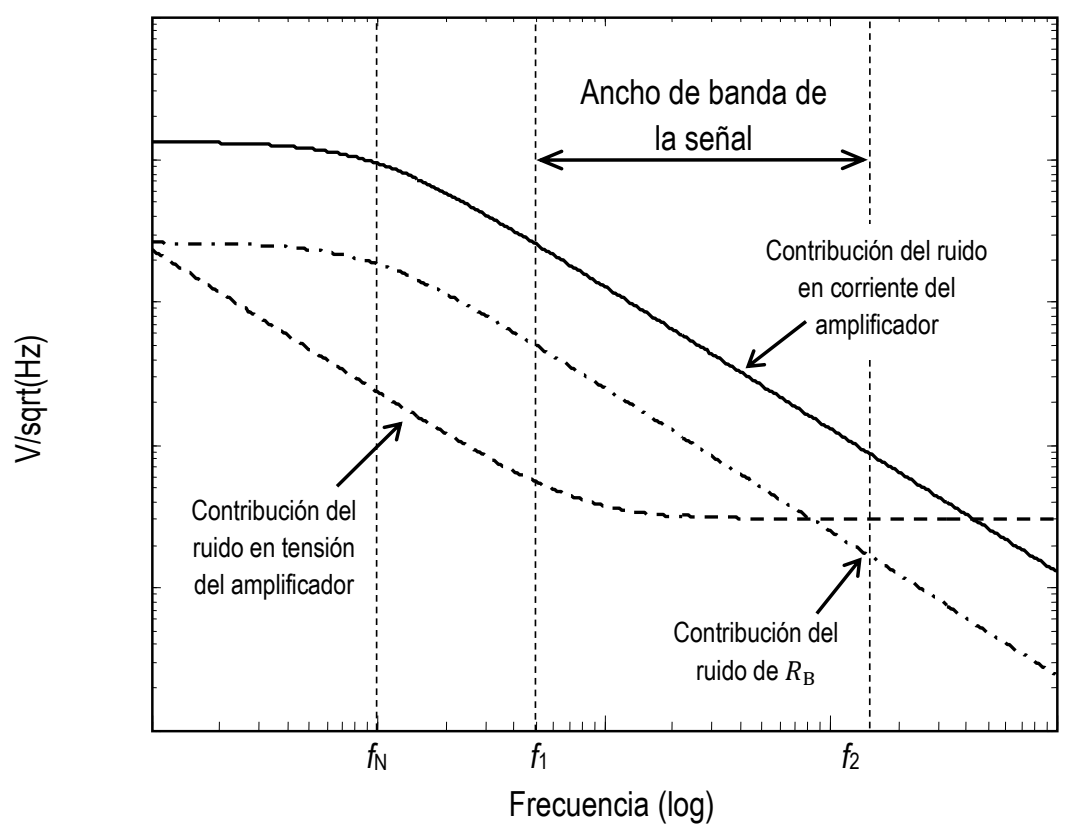

Figura 3-12: Diferentes contribuciones a la densidad espectral de potencia de ruido a la salida del buffer de electrodo: corriente de ruido del amplificador (línea llena), tensión de ruido del amplificador (línea troceada) y ruido del resistor de polarización (línea troceada y punteada).

Una curva típica del ruido total a la salida del electrodo y las contribuciones de cada término en (3-4) se muestra en la Figura 3-12. En bajas frecuencias, los aportes de $e_{\mathrm{R}}$ y de $i_{\mathrm{n}} R_{\text {Bias }}$ son muy altos, del orden de 100 $\mu \mathrm{V} / \sqrt{\mathrm{Hz}}$, para $R_{\text {Bias }}=1 \mathrm{~T} \Omega$ e $i_{\mathrm{n}} \tan$ baja como $0.1 \mathrm{fA} / \sqrt{\mathrm{Hz}}$. Para reducir estas contribuciones de ruido a valores aceptables, la frecuencia de corte $f_{\mathrm{N}}$ debe estar considerablemente por debajo del ancho de banda de la señal. En dicho rango de frecuencias (3-4) puede ser aproximada por:

$$
e_{\mathrm{O}}^{2}(f) \approx e_{\mathrm{R}}^{2}\left(\frac{f_{\mathrm{N}}}{f}\right)^{2}+i_{\mathrm{n}}^{2} R_{\text {Bias }}^{2}\left(\frac{f}{f_{\mathrm{N}}}\right)^{2}+e_{\mathrm{n}}^{2}
$$

Reemplazando $e_{\mathrm{R}}$ por la expresión de Nyquist $\left(e_{\mathrm{R}}^{2}=4 k T R_{\mathrm{B}}\right)$ y $f_{\mathrm{N}}$ por (3-3) resulta en

$$
e_{\mathrm{O}}^{2} \approx \frac{k T}{\left(\pi C_{\mathrm{S}} f\right)^{2}} \frac{1}{R_{\text {Bias }}}+\frac{i_{\mathrm{n}}^{2}}{\left(2 \pi C_{\mathrm{S}} f\right)^{2}}+e_{\mathrm{n}}^{2}
$$




$$
e_{\mathrm{O}}^{2}(f) \approx \frac{1}{\left(\pi C_{\mathrm{S}} f\right)^{2}}\left(\frac{k T}{R_{\text {Bias }}}+\frac{i_{\mathrm{n}}^{2}}{4}\right)+e_{\mathrm{n}}^{2}
$$

El último término, debido al ruido en tensión del amplificador operacional, también está presente en mediciones realizadas con electrodos activos húmedos y secos (Huigen et al. 2002, Matthews et al. 2005). El primer término, por otra parte, decrece con la frecuencia y puede verse como un "exceso de ruido" ante el uso de electrodos húmedos o secos. Esta expresión considera el ruido propio del electrodo, cuando se mide a través de prendas de vestir, las componentes resistivas de la tela que oficia de dieléctrico también puede contribuir con ruido a la medición (Chi et al. 2010).

Dependiendo del valor del resistor $R_{\mathrm{Bias}}$, uno de los dos términos dentro del paréntesis de (3-7) dominará. La contribución del ruido del resistor $R_{\text {Bias }}$ igualará a la de la corriente de ruido del amplificador para el valor $R_{\text {Bias }}=$ $R_{\text {Bias,C }}$ dado por

$$
R_{\text {Bias, } \mathrm{C}}=\frac{4 k T}{i_{\mathrm{n}}^{2}}
$$

En general, para $R_{\text {Bias }}$ menor a decenas de G $\Omega$ dominará la contribución de ruido del resistor, de acuerdo con (3-7), el ruido a la salida del buffer decrece con $R_{\text {Bias }}$ como se reporta en (Prance et al. 2000). Por otro lado, para $R_{\text {Bias }}$ de unos pocos $\mathrm{T} \Omega$ o más, el aporte del ruido del resistor es despreciable frente a la contribución de la corriente de ruido del amplificador, incluso con $i_{\mathrm{n}}$ tan bajos como $0.1 \mathrm{fA} / \sqrt{\mathrm{Hz}}$, como fue establecido por (Sullivan et al. 2007, Matthews et al. 2005).

\subsubsection{Efecto de la guarda activa}

El efecto colateral de la utilización de una guarda activa es una amplificación del ruido en tensión $e_{\mathrm{n}}$ del buffer por un factor $\left(1+\frac{C_{\mathrm{SH}}}{C_{\mathrm{S}}}\right)$, donde $C_{\mathrm{SH}}$ representa la capacitancia desarrollada entre el nodo de guarda y el de medida (que también incluye la capacidad de entrada de modo diferencial del amplificador).

$$
e_{\mathrm{O}}^{2}(f) \approx \frac{1}{\left(\pi C_{\mathrm{S}} f\right)^{2}}\left(\frac{k T}{R_{\mathrm{Bias}}}+\frac{i_{\mathrm{n}}^{2}}{4}\right)+e_{\mathrm{n}}^{2}\left(1+\frac{C_{\mathrm{SH}}}{C_{\mathrm{S}}}\right)^{2}
$$

En la Figura 3-13 puede verse un ejemplo de cómo se ha implementado la guarda activa en un electrodo capacitivo para EMG construido en un circuito impreso de dos capas.

(a)

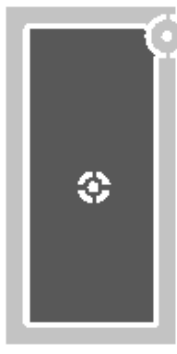

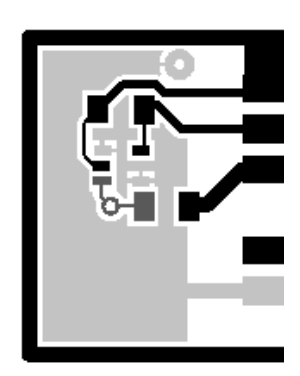

(b)

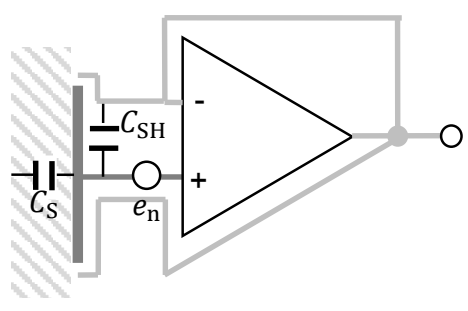

Figura 3-13: (a): capa inferior en contacto con el sujeto (izquierda) y superior (derecha) del circuito impreso sobre el cual se construyó el electrodo capacitivo para EMG. En gris oscuro se observa el nodo de sensado, en gris claro la guarda activa y salida del 
electrodo, en negro otros nodos del circuito. (b): Esquemático donde se puede observar el efecto de $C_{\mathrm{SH}}$ y $C_{\mathrm{S}}$ en la realimentación positiva del ruido en tensión $e_{\mathrm{n}}$.

\subsection{Electrodo capacitivo desarrollado para EMG}

Las características en frecuencia de las señales de EMG, cuya frecuencia de corte inferior usualmente suele tomarse entre 10 y $30 \mathrm{~Hz}$, relajan los requerimientos de diseño de electrodos capacitivos. Principalmente para la resistencia $R_{\mathrm{Bias}}$, que puede ser de algunos $\mathrm{G} \Omega$. Suponiendo que se escoge un resistor comercial de $10 \mathrm{G} \Omega$ y que la capacidad de acoplamiento $C_{\mathrm{S}}$ desarrolla unos modestos $50 \mathrm{pF}$, obtenemos una frecuencia de corte de $3 \mathrm{~dB}$ de tan solo 1,6Hz. Como se demuestra en (Spinelli y Haberman 2010), es deseable que dicha frecuencia de acoplamiento dada por $\mathrm{R}_{\mathrm{Bias}}$ y $\mathrm{C}_{\mathrm{S}}$ sea considerablemente menor a la frecuencia inferior de la señal de interés a fin de reducir la tensión eficaz de ruido.

Así mismo, el bajo valor de $\mathrm{R}_{\mathrm{Bias}}$ hará que el ruido propio del resistor domine frente al de corriente del amplificador, de la misma manera que el efecto de la corriente de polarización del amplificador se hace despreciable, aunque si producirán una tensión de offset de algunos $\mathrm{mV}$ que de ninguna manera comprometerán el rango de entrada del amplificador. Esto si puede ocurrir cuando se utilizan $\mathrm{R}_{\text {Bias }}$ del orden de los $\mathrm{T} \Omega$

Se desarrolló una placa de circuito impreso doble faz que consta de 2 electrodos capacitivos con ganancia unitaria. Si bien cada electrodo es un circuito independiente y desacoplado, la ubicación cercana de ambos electrodos permitió ubicarlos sobre un mismo soporte físico reduciendo la cantidad de cables y favoreciendo su colocación.

Dada la similitud de ambos electrodos, se decidió no incluir un circuito de neutralización de la capacidad de entrada que es de unos $5 \mathrm{pF}$. Los circuitos de neutralización de la capacidad de entrada son comúnmente utilizados en electrodos capacitivos, ya reducen el valor de la capacidad de entrada mediante la realimentación positiva de la señal medida. Dicho mecanismo de neutralización se encuentra desarrollarlo en las siguientes referencias (Amatniek 1958, Spinelli y Haberman 2010).

El diseño se basa en dos buffers construidos con operacionales OPA320, cuyos parámetros de interés se resumen en la Tabla 3-2. Los resistores de polarización escogidos son de $10 \mathrm{G} \Omega$, comerciales de montaje superficial, y conectan los nodos de censado a un potencial de referencia de DC. Los componentes son todos de montaje superficial, ubicados en la cara superior de la placa de circuito impreso. Los planos de sensado de los electrodos, en la cara inferior de la placa, son de geometría rectangular de $9.5 \mathrm{~mm}$ por $19 \mathrm{~mm}$ y los centros de ambos electrodos se encuentran separados por $20 \mathrm{~mm}$. Utilizando como dieléctrico polipropileno de aproximadamente $60 \mu \mathrm{m}$ y apoyándolo directamente sobre la piel del sujeto, se logran capacidades de acoplamiento $C_{\mathrm{S}}$ del orden de los $50 \mathrm{pF}$. Si el electrodo sensa los biopotenciales a través de una prenda de algodón del orden de los $300 \mu \mathrm{m}$ dicho valor de capacidad se reduce a unos 3-4 pF.

El esquema de los electrodos capacitivos, conectados al $A B$ y el resto del sistema de adquisición se puede observar en la Figura 3-14. En dicha figura se observa que el tercer electrodo no es capacitivo sino húmedo 0 seco, y está conectado a GND para fijar una referencia de modo común. Dado que el acoplamiento del electrodo es en $\mathrm{AC}$, el potencial de continua del sujeto puede diferir de $V_{\mathrm{REF}}$. 


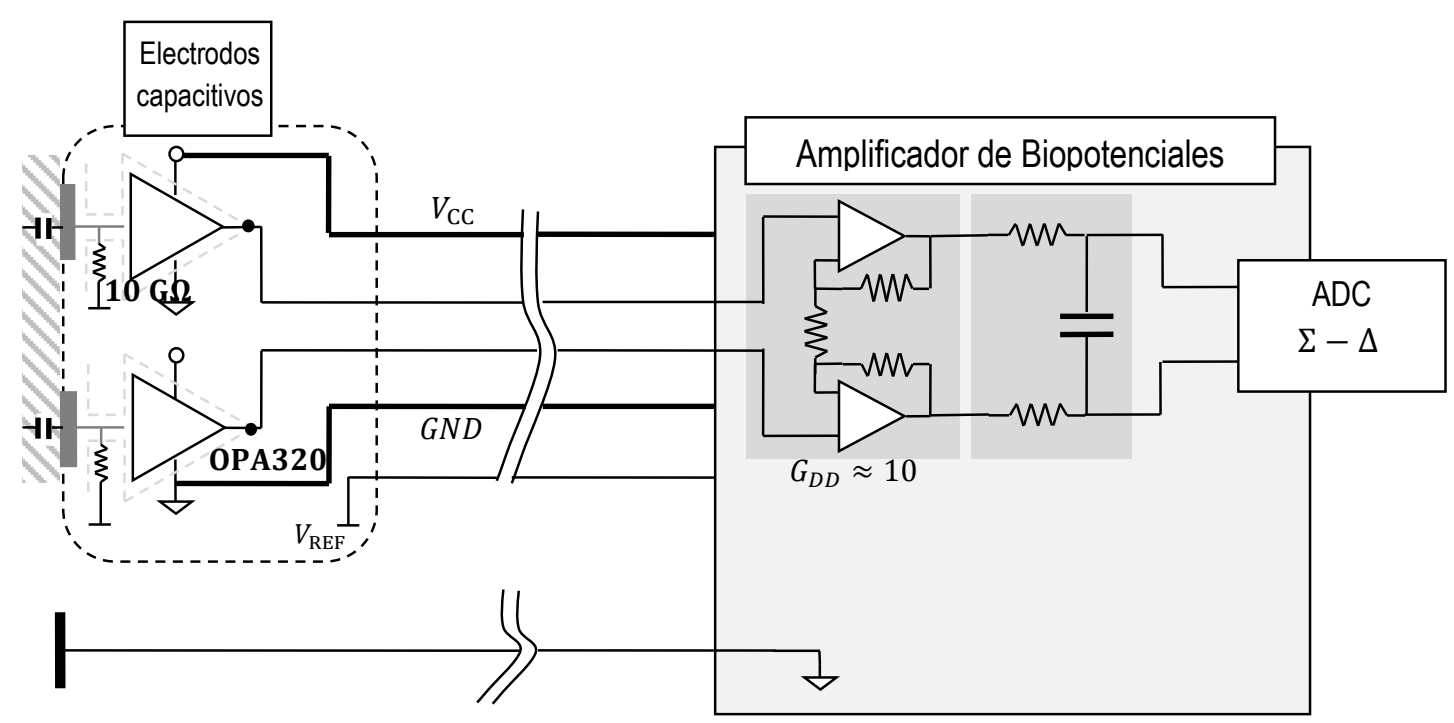

Figura 3-14: Esquema del par de electrodos capacitivos desarrollados para EMG y del $A B$ asociado.

\begin{tabular}{|c|c|}
\hline Parámetro & Valor \\
\hline$i_{\mathrm{n}}$ & $0.6 \mathrm{fA} / \sqrt{\mathrm{Hz}}$ \\
\hline$e_{\mathrm{n}}(10 \mathrm{~Hz})$ & $60 \mathrm{nV} / \sqrt{\mathrm{Hz}}$ \\
\hline$e_{\mathrm{n}}(10 \mathrm{~Hz})$ & $20 \mathrm{nV} / \sqrt{\mathrm{Hz}}$ \\
\hline$i_{\text {Bias }}$ & $\pm 0.2 \mathrm{pA}$ \\
\hline$C_{\text {in }}$ & $4 \mathrm{pF}$ \\
\hline$C_{\mathrm{D}}\left(\right.$ parte de $\left.C_{\mathrm{SH}}\right)$ & $5 \mathrm{pF}$ \\
\hline
\end{tabular}

Tabla 3-2: Parámetros del amplificador operacional OPA320, de interés para el diseño del electrodo capacitivo.

\subsubsection{Estimación de Ruido}

Utilizando la fórmula (3-9), considerando $R_{\text {Bias }}=10 \mathrm{G} \Omega, C_{\mathrm{S}} \approx 50 \mathrm{pF}, C_{\mathrm{SH}} \approx 10 \mathrm{pF}, e_{\mathrm{n}} \approx 40 \mathrm{nV} / \sqrt{\mathrm{Hz}} \mathrm{e}$ $i_{\mathrm{n}}=0.6 \mathrm{fA} / \sqrt{\mathrm{Hz}}$, e integrando en un ancho debanda de $30 \mathrm{~Hz}$ a $380 \mathrm{~Hz}$ se obtiene un nivel de ruido aproximado de unos $1.2 \mu \mathrm{V}_{\mathrm{RMS}}$ por electrodo capacitivo. Teniendo en cuenta que la señal obtenida es la diferencia entre dos electrodos capacitivos del mismo tipo, el ruido en la señal diferencial estimado será de $\sqrt{2} x 1.2 \mu \mathrm{V}_{\mathrm{RMS}}=$ $1.7 \mu \mathrm{V}_{\mathrm{RMS}}$, que es aceptable para señales de EMG. 


\subsubsection{Resultados Experimentales}

\subsubsection{Ruido y señales de EMG adquiridas sobre la piel}

Se realizaron mediciones con electrodos capacitivos colocados sobre el antebrazo y sobre el músculo facial cigomático mayor. En ambos casos se aprecia un $90 \%$ más de ruido base que el estimado analíticamente utilizando (3-9) lo cual puede deberse a dispersión en los parámetros considerados para la estimación, principalmente para los valores de $C_{\mathrm{S}}$ y $C_{\mathrm{SH}}$, así también como a EMG residual. En la Figura 3-15 se aprecia la densidad espectral de potencia del ruido base (señal medida con el músculo cigomático mayor en reposo) junto con las densidades de ruido estimadas para diferentes combinaciones de $C_{\mathrm{S}}$ y $C_{\mathrm{SH}}$.

En la Figura 3-16 y Figura 3-17, se puede observar registros de 2 segundos sobre el músculo cigomático mayor y sobre el antebrazo, respectivamente. En ambos casos, durante el primer segundo se mantuvieron los músculos en reposo para luego contraerlos. Se observa que durante el reposo (una vez eliminada la interferencia aditiva de $50 \mathrm{~Hz}$ ) ambos registros muestran el mismo nivel de ruido base de unos $3 \mu \mathrm{V}_{\mathrm{RMS}}$ y aproximadamente $\pm 10 \mu \mathrm{V}$ de amplitud. Con estos niveles tan bajos de ruido es posible lograr muy buenas relaciones señal a ruido aun con músculos pequeños como los faciales, que para contracciones débiles producen señales de tan solo decenas de microvolts de amplitud.

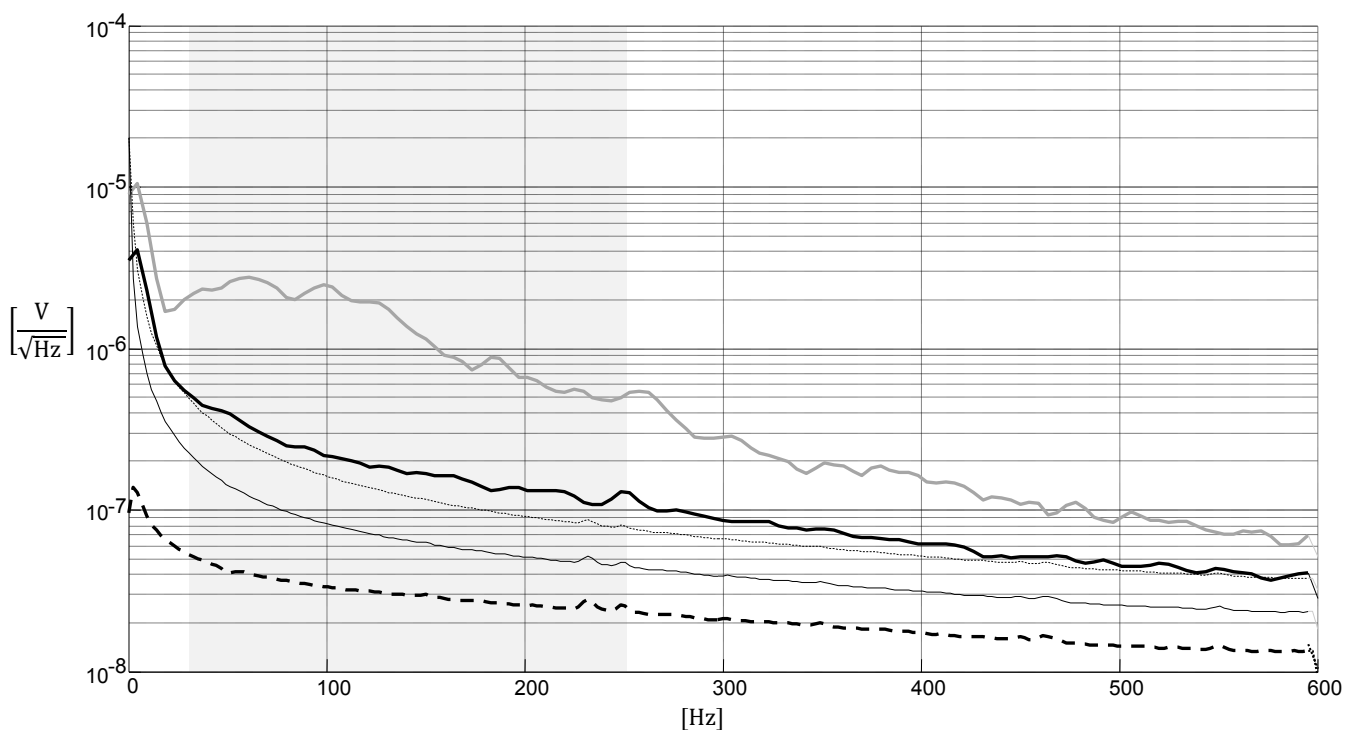

Figura 3-15: Densidad espectral de potencia en unidades de $[V / \sqrt{\mathrm{Hz}}]$. Señal diferencial medida entre ambos electrodos capacitivos con el rostro en reposo (línea gruesa negra). Señal diferencial medida entre ambos electrodos capacitivos con una leve contracción del músculo cigomático mayor (línea gruesa gris). Estimación de ruido considerando $C_{\mathrm{S}}=50 \mathrm{pf}$ y $C_{\mathrm{SH}}=10 \mathrm{pf}$ (línea angosta negra). Estimación de ruido considerando $C_{\mathrm{S}}=25 \mathrm{pf}$ y $C_{\mathrm{SH}}=20 \mathrm{pf}$ (línea angosta troceada negra). Medición de ruido cortocircuitando a la referencia ambos electrodos (línea gruesa troceada negra). El área gris indica el ancho de banda de interés. 


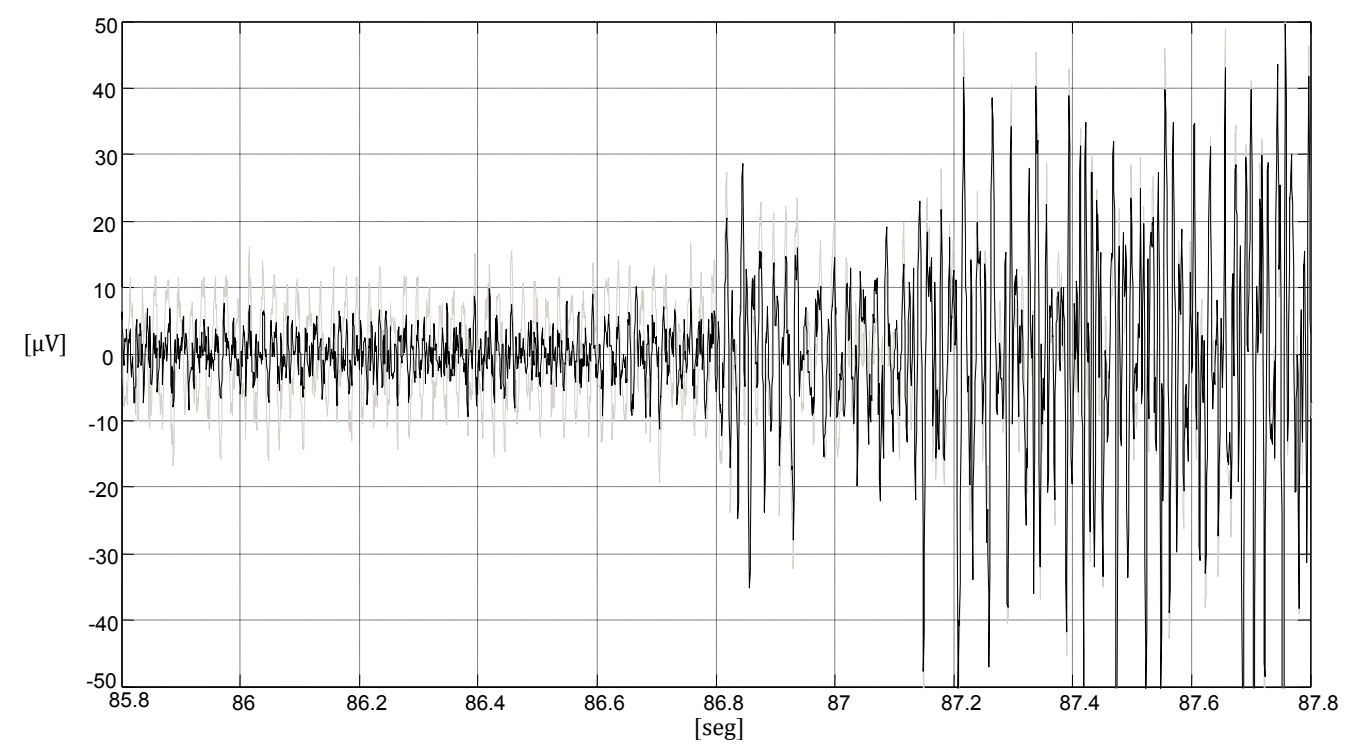

Figura 3-16: Segmento de 2 segundos de señal, adquirida con los electrodos capacitivos sobre el músculo cigomático mayor. Durante el primer segundo el músculo permanece en reposo y luego se contrae levemente. La señal cruda ha sido pasada por un filtro pasabanda entre $30 \mathrm{~Hz}$ y $250 \mathrm{~Hz}$ (línea gris) y a esta última se le ha aplicado un filtro notch centrado en $50 \mathrm{~Hz}$ para eliminar la interferencia (línea negra).

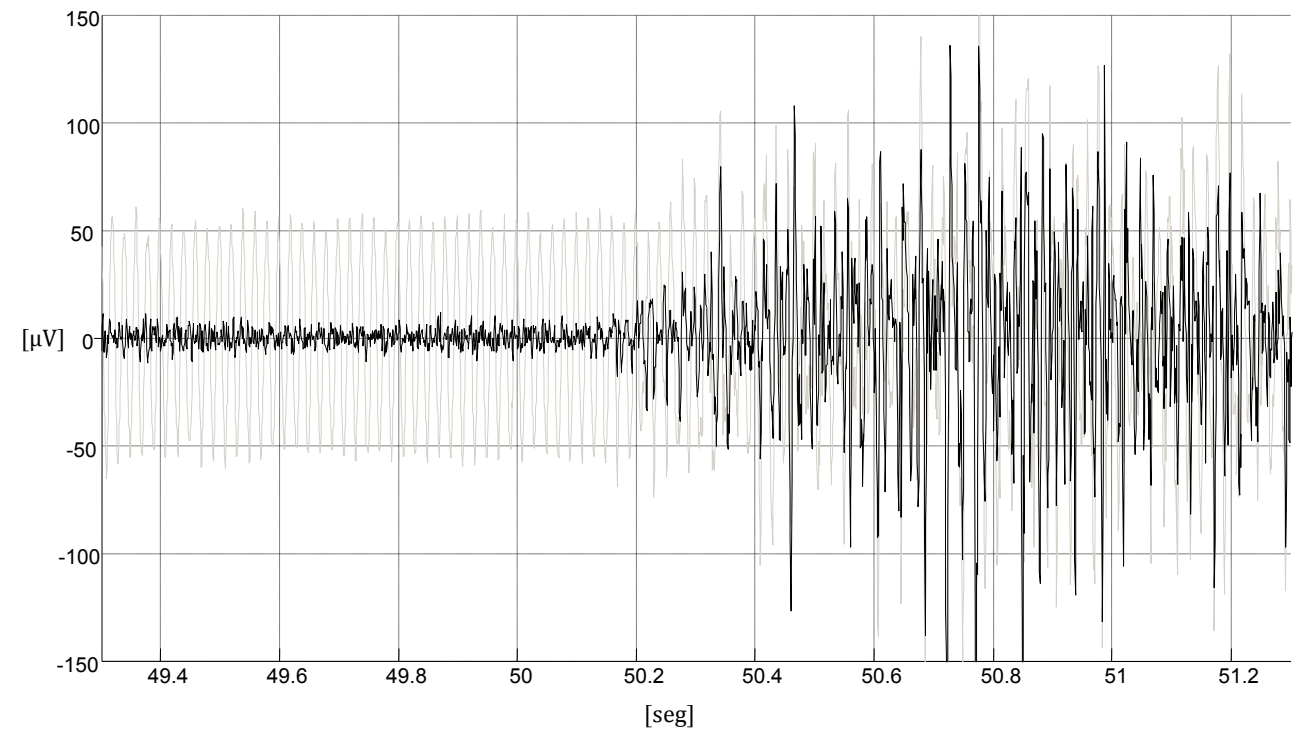

Figura 3-17: Segmento de 2 segundos de señal, adquirida con los electrodos capacitivos sobre el antebrazo. Durante el primer segundo el antebrazo permanece en reposo y luego se contrae levemente. La señal cruda ha sido pasada por un filtro pasabanda entre $30 \mathrm{~Hz}$ y 250 $\mathrm{Hz}$ (línea gris) y a esta última se le ha aplicado un filtro notch centrado en $50 \mathrm{~Hz}$ para eliminar la interferencia (línea negra).

Cómo se puede observar en dichas figuras, la interferencia de $50 \mathrm{~Hz}$ es significativa. El rechazo de este tipo de interferencia puede mejorar mucho si se neutraliza la capacidad de entrada de modo común mediante un esquema 
de realimentación positiva (Spinelli y Haberman 2010) y de esta manera reducir el efecto divisor de potencial. Otro camino es la realimentación de la señal de modo común al sujeto como se verá en el Capítulo 4 reduciendo directamente la fuente de interferencia.

\subsubsection{Señales de EMG adquiridas sobre una prenda de algodón}

Se realizaron dos registros diferentes midiendo a través de prendas de algodón sobre el bíceps y el antebrazo. En el primer caso se obtiene un ruido base de $13 \mu \mathrm{V}_{\mathrm{RMS}}$ sobre el bíceps (ver Figura 3-18), mientras que en el segundo caso $20 \mu \mathrm{V}_{\mathrm{RMS}}$ sobre el antebrazo (ver Figura 3-19). La variación en estos valores es esperable dado que se utilizaron distintas prendas.

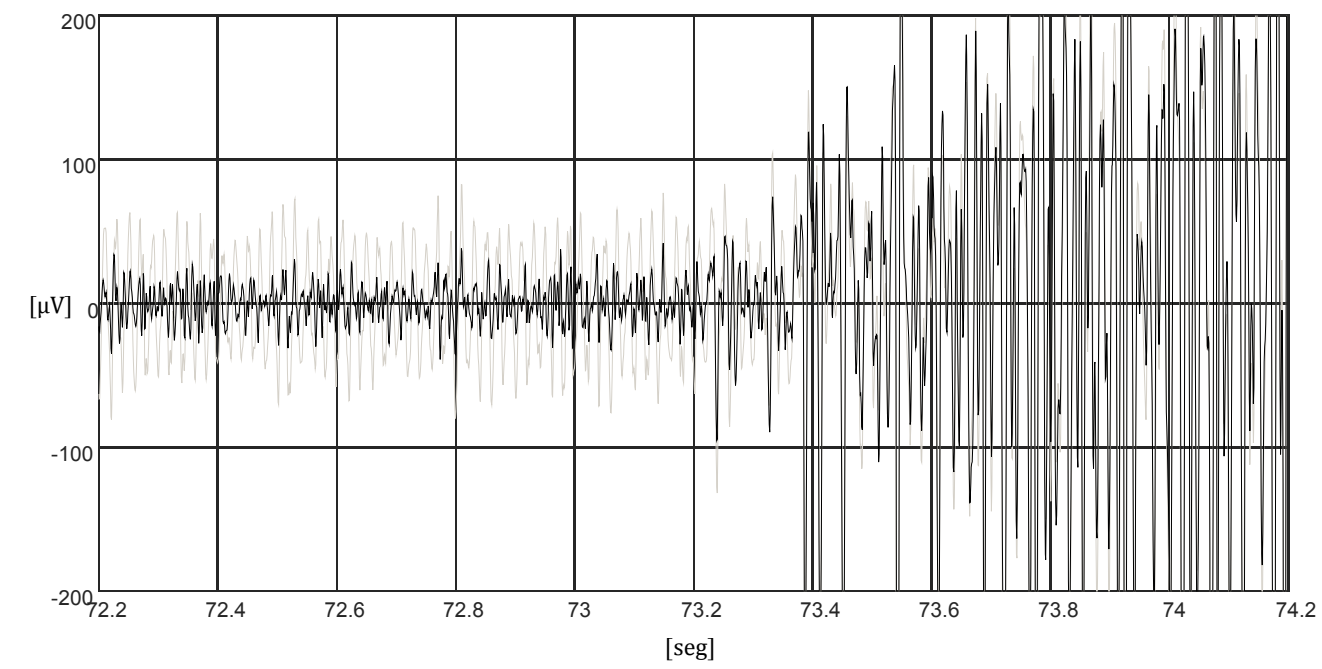

Figura 3-18: Segmento de 2 segundos de señal, adquirida con los electrodos capacitivos sobre el bíceps y sobre una prenda de algodón. Durante el primer segundo el antebrazo permanece en reposo y luego se contrae levemente. La señal cruda ha sido pasada por un filtro pasabanda entre $30 \mathrm{~Hz}$ y $250 \mathrm{~Hz}$ (línea gris) y a esta última se le ha aplicado un filtro notch centrado en $50 \mathrm{~Hz}$ para eliminar la interferencia (línea negra). 


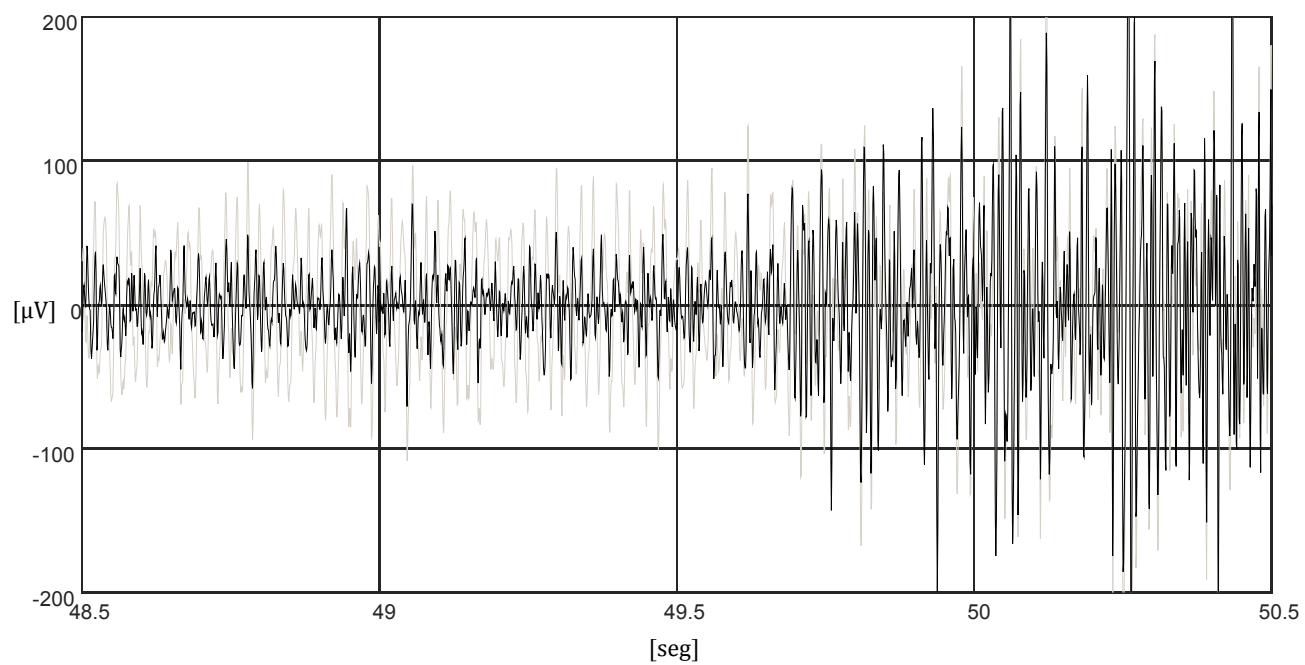

Figura 3-19: Segmento de 2 segundos de señal, adquirida con los electrodos capacitivos sobre el antebrazo y una prenda de algodón. Durante el primer segundo el antebrazo permanece en reposo y luego se contrae levemente. La señal cruda ha sido pasada por un filtro pasabanda entre $30 \mathrm{~Hz}$ y $250 \mathrm{~Hz}$ (línea gris) y a esta última se le ha aplicado un filtro notch centrado en $50 \mathrm{~Hz}$ para eliminar la interferencia (línea negra).

\subsection{Conclusiones}

Los electrodos son un componente fundamental en la adquisición de biopotenciales, y una mala elección de los mismos puede perjudicar irreversiblemente el registro de las señales biológicas.

Entre los distintos tipos de electrodos analizados, en lo que respecta a la adquisición de EMG, se concluye que luego de un par de minutos los electrodos secos presentan el mismo desempeño que los húmedos, si se utilizan electrodos activos.

Teniendo en cuenta las ventajas de los electrodos secos sobre los húmedos en usos prolongados, se justifica la utilización de electrodos secos en dispositivos de asistencia que requieran extensos períodos de utilización por parte de los usuarios, siempre y cuando se adapte la impedancia, esto es, que los electrodos sean secos y activos. La principal desventaja de esta elección es la necesidad de un soporte mecánico que fije el electrodo a la piel del sujeto. Vale aclarar también que el desempeño de los electrodos secos dependerá del material de los mismos y que las conclusiones y resultados experimentales aplican a electrodos secos de acero inoxidable.

En cuanto a la utilización de electrodos capacitivos, se concluye que es una técnica viable para ser considerada en DAs controladas por EMG. La versatilidad lograda con este tipo de electrodos para registrar señales a través de materiales dieléctricos como prendas de vestir los hace ideales para DAs. Además, esta tecnología hace posible el desarrollo de equipos completamente aislados del usuario, es decir sin contacto galvánico, lo que incrementa las condiciones de seguridad, facilitando también la comercialización y aceptación por parte de los usuarios. El punto débil de este tipo de electrodos son los mayores niveles de ruido, que demandan capacidades de acoplamiento más altas, implicando mayor área de electrodo (si se mantiene el espesor del dieléctrico). Esto último degrada la selectividad en las señales de EMG y hace a los electrodos capacitivos más voluminosos que sus equivalentes secos 
Capítulo 3 - Electrodos para Dispositivos de Ayuda 


\section{Capítulo 4}

\section{Reducción de interferencia de modo común}

En el presente capítulo se presenta la técnica de reducción de la interferencia de modo común a partir de la realimentación negativa de $v_{\mathrm{CM}}$. Esta técnica consiste en la medición o estimación de la tensión $v_{\mathrm{CM}}$, su amplificación y la realimentación mediante un electrodo adicional. El nombre del circuito que realiza esta función (DRL: Driven Right Leg) proviene de su utilización en la práctica del electrocardiograma, donde el electrodo de realimentación es colocado en la pierna derecha.

Dadas algunas limitaciones en el circuito DRL clásico se propone una mejora del mismo que incorpora circuitos sintonizados a la frecuencia de la interferencia. Se muestra una implementación digital del mismo (DDRL) y resultados experimentales que muestran que la reducción de este circuito es tan alta que permite incluso utilizar simples amplificadores Single-Ended en la captura de señales de EEG.

La adquisición de biopotenciales mediante electrodos capacitivos sufre particularmente el efecto de la interferencia por modo común dado el bajo CMRR de esta tecnología, requiriendo un circuito de realimentación de modo común. Se analiza la estabilidad de este tipo de sistemas para lograr un DRL capacitivo (CDRL) estable y con la suficiente ganancia para rechazar la interferencia. Se presenta la implementación de un prototipo, acompañada por resultados experimentales en la adquisición de ECG y EMG.

Se presenta también la implementación de un sensor de EMG que integra tanto amplificación para un canal diferencial como el circuito DRL sobre los mismos electrodos. 


\subsection{Reducción de $v_{\mathrm{CM}}$ por realimentación negativa}

Como fue tratado en el Capítulo 2, la tensión de modo común $v_{\mathrm{CM}}$ interfiere aditivamente a la señal biopotencial que se desea adquirir a través de transformaciones al modo diferencial. La manera de cuantificar el grado en que $v_{\mathrm{CM}}$ afecta a la señal medida es mediante el rechazo de modo común equivalente $C M R R_{\mathrm{E}}$, que resume en un único valor los diversos mecanismos de conversión de modo existentes en las distintas etapas de procesamiento de la señal. Esto se observa en (4-1), donde $v_{\mathrm{i}}$ es la señal obtenida, compuesta por la señal biopotencial $v_{\mathrm{s}}$ y el término de interferencia por modo común, donde se descartan los otros mecanismos de interferencia. Lógicamente, es deseable contar con el mayor $C M R R_{\mathrm{E}}$ posible.

$$
v_{\mathrm{i}}=v_{\mathrm{s}}+\frac{v_{\mathrm{CM}}}{C M R R_{\mathrm{E}}}
$$

Como se vio también en dicho capítulo, el uso de amplificadores de instrumentación diferenciales con $C M R R_{\mathrm{AB}}>90 \mathrm{~dB}$ es suficiente para que el rechazo de modo común equivalente esté dominado por el efecto divisor de potencial. Esto ocurre particularmente con el uso de electrodos secos donde los valores de impedancia de electrodo suelen ser más altos.

$$
C M R R_{\mathrm{E}}=\left[\frac{1}{C M R R_{\mathrm{AB}}}+\frac{1}{C M R R_{\mathrm{DP}}}\right]^{-1} \approx C M R R_{\mathrm{DP}}
$$

En la adquisición de biopotenciales para su utilización en DAs, donde se prefiere la utilización de electrodos secos, el $C M R R_{\mathrm{DP}}$ se ve degradado. A su vez, las condiciones de EMI no controladas pueden conducir a una combinación de capacidades parásitas que producen altos valores de $v_{\mathrm{CM}}$. En estos casos el rechazo de la tensión de modo común no suele ser suficiente y el término interferente en (4-1) podría incluso superar al de la señal.

Es así como, frente a un rechazo de modo común insuficiente para la posibles magnitudes alcanzadas por $v_{\mathrm{CM}}$, se hace necesario reducir directamente la señal interferente $v_{\mathrm{CM}}$. Una técnica ampliamente utilizada en la instrumentación de biopotenciales se basa en la realimentación negativa de la señal de modo común mediante un circuito Driven Right Leg (DRL) (Winter y Webster 1983a).

Para poder realizar un análisis de dicho circuito, en la Figura 4-1, se recuerda el modelo que explica la aparición de $v_{\mathrm{CM}}$. La expresión (2-6) muestra la dependencia del valor de $v_{\mathrm{CM}}$ tanto con la corriente $i_{\mathrm{EG}}$, que atraviesa el electrodo de referencia, como con la impedancia de este electrodo: $Z_{\mathrm{EG}}$.

$$
v_{\mathrm{CM}}=i_{\mathrm{EG}} \cdot Z_{\mathrm{EG}}=\frac{V_{\mathrm{PL}} \cdot\left|\frac{C_{\mathrm{P}}}{C_{\mathrm{P}}+C_{\mathrm{B}}}-\frac{C_{\mathrm{SUP}}}{C_{\mathrm{SUP}}+C_{\mathrm{ISO}}}\right|}{\sqrt{\left[\left(\frac{1}{C_{\mathrm{P}}+C_{\mathrm{B}}}+\frac{1}{C_{\mathrm{SUP}}+C_{\mathrm{ISO}}}\right) \frac{1}{2 \pi f_{\mathrm{PL}}}\right]^{2}+Z_{\mathrm{EG}}^{2}}} Z_{\mathrm{EG}}
$$

Si bien en la definición de $i_{\mathrm{EG}}$ interviene $Z_{\mathrm{EG}}$, a fines prácticos dicha corriente depende solo de las capacidades parásitas del modelo, dado que a la frecuencia de red $(50-60 \mathrm{~Hz})$ la reactancia de estas capacidades es mucho mayor que los posibles valores de $Z_{\mathrm{EG}}$. La expresión aproximada para $i_{\mathrm{EG}}$ se aprecia en (4-4). 


$$
i_{\mathrm{EG}}=\frac{V_{\mathrm{PL} \cdot}\left|\frac{C_{\mathrm{P}}}{C_{\mathrm{P}}+C_{\mathrm{B}}}-\frac{C_{\mathrm{SUP}}}{C_{\mathrm{SUP}}+C_{\mathrm{ISO}}}\right|}{\sqrt{\left[\left(\frac{1}{C_{\mathrm{P}}+C_{\mathrm{B}}}+\frac{1}{C_{\mathrm{SUP}}+C_{\mathrm{ISO}}}\right) \frac{1}{2 \pi f_{\mathrm{PL}}}\right]^{2}+Z_{\mathrm{EG}}^{2}}} \approx \frac{V_{\mathrm{PL}} \cdot\left|\frac{C_{\mathrm{P}}}{C_{\mathrm{P}}+C_{\mathrm{B}}}-\frac{C_{\mathrm{SUP}}}{C_{\mathrm{SUP}}+C_{\mathrm{ISO}}}\right|}{\left(\frac{1}{C_{\mathrm{P}}+C_{\mathrm{B}}}+\frac{1}{C_{\mathrm{SUP}}+C_{\mathrm{ISO}}}\right) \frac{1}{2 \pi f_{\mathrm{PL}}}}
$$

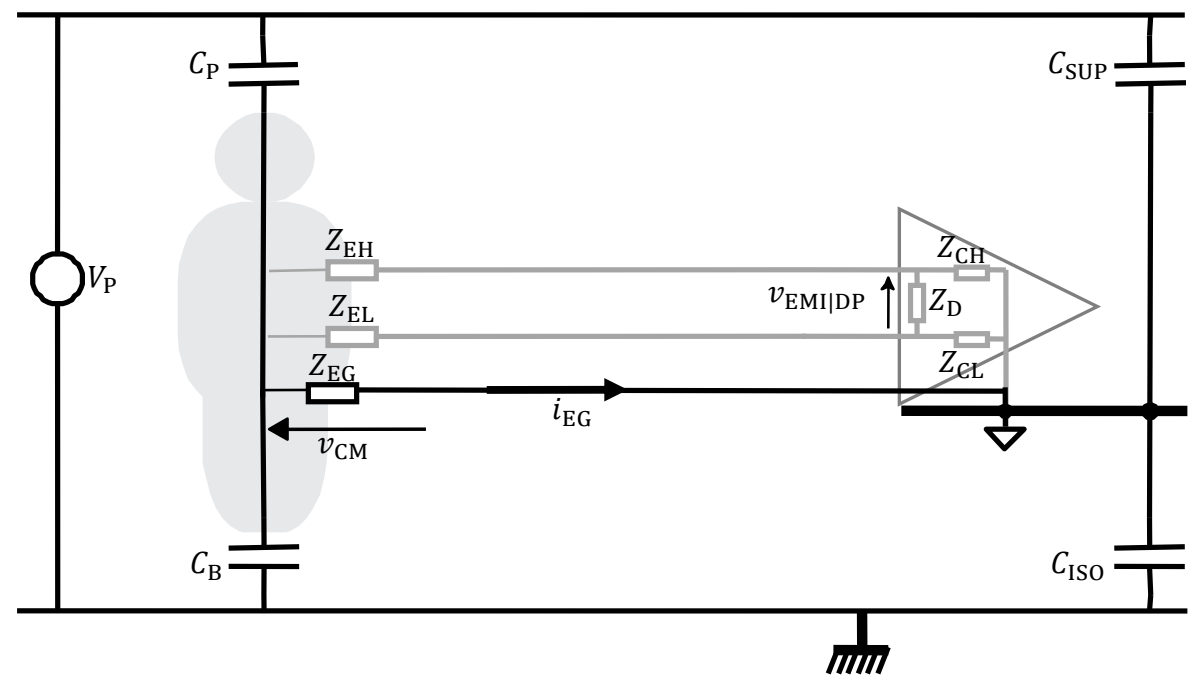

Figura 4-1: Modelo de EMI simplificado que explica la formación de una tensión de Modo Común en el sistema. Nótese que $\boldsymbol{v}_{\mathrm{CM}}$ depende solo de las capacidades de acoplamiento $\boldsymbol{C}_{\mathbf{P}}, \boldsymbol{C}_{\mathbf{B}}, \boldsymbol{C}_{\mathbf{S U P}}$ y $\boldsymbol{C}_{\mathbf{I S O}}$, y de la impedancia del electrodo de referencia $\boldsymbol{Z}_{\mathbf{E G}}$. Se observa la tensión de interferencia $\boldsymbol{v}_{\mathbf{E M I} \mid \mathbf{D P}}$ a la entrada del $\mathrm{AB}$ producida por el efecto divisor de potencial.

El circuito del DRL mide la tensión de modo común y la realimenta negativamente, con una ganancia $G_{\mathrm{DRL}}$ a través del que anteriormente era el electrodo de referencia (ver Figura 4-2).

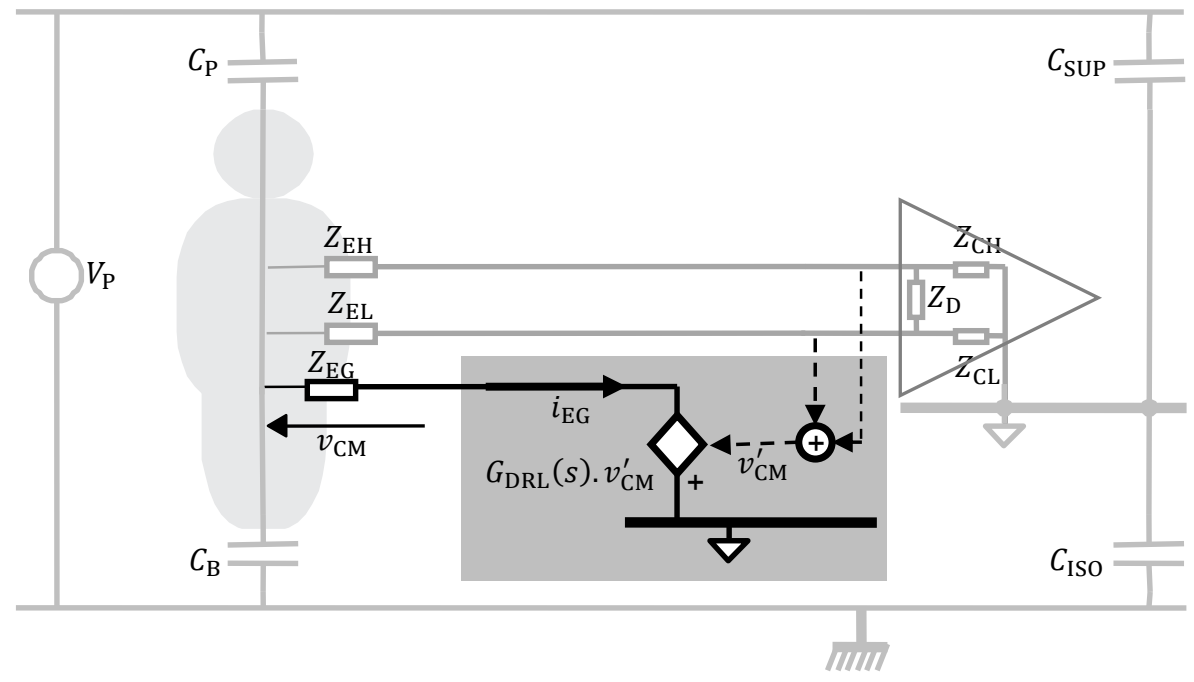

Figura 4-2: Esquema que muestra cómo opera el circuito DRL, tomando una medida $v_{\mathrm{CM}}^{\prime} \approx v_{\mathrm{CM}}$ y realimentándola negativamente, amplificada por $G_{\mathrm{DRL}}(s)$, a través del electrodo de referencia. 
Observando el esquema de la Figura 4-2, La fórmula (2-6) se reformula de la siguiente manera:

$$
v_{\mathrm{CM}}=i_{\mathrm{EG}} \cdot Z_{\mathrm{EG}}-G_{\mathrm{DRL}} v_{\mathrm{CM}}^{\prime}
$$

Si consideramos que para $50 \mathrm{~Hz}$ las impedancias de entrada de modo común son mucho mayores que las impedancias de los electrodos, podemos decir que la tensión de modo común estimada $v_{\mathrm{CM}}^{\prime}$ coincide con la real $\left(v_{\mathrm{CM}}^{\prime} \cong v_{\mathrm{CM}}\right)$. Reemplazando en (4-5) y operando se obtiene:

$$
v_{\mathrm{CM}}=\frac{i_{\mathrm{EG}} \cdot Z_{\mathrm{EG}}}{1+G_{\mathrm{DRL}}}=i_{\mathrm{EG}} \cdot Z_{\mathrm{EG}}^{\prime}
$$

Donde se observa como $v_{\mathrm{CM}}$ es reducida $1+G_{\mathrm{DRL}}$ veces gracias a la realimentación negativa. Esto puede interpretarse como una reducción del valor de la impedancia del electrodo de $Z_{\mathrm{EG}}$ a $Z_{\mathrm{EG}}^{\prime}=$ $Z_{\mathrm{EG}} /\left(1+G_{\mathrm{DRL}}\right)$. Lo anterior muestra que son deseables valores altos de $G_{\mathrm{DRL}}$ para atenuar el valor de modo común, al menos en la frecuencia de red, donde está concentrada la mayor parte de la interferencia.

Las distintas implementaciones del circuito DRL en general difieren en dos factores: como se estima $v_{\mathrm{CM}}$, y cómo se diseña la transferencia $G_{\mathrm{DRL}}(s)$.

\subsubsection{Estimación de $v_{\mathrm{CM}}$}

La tensión $v_{\mathrm{CM}}$ puede estimarse promediando las señales de todos los electrodos utilizados para la medición de biopotenciales (Winter y Webster 1983a, Metting Van Rijn et al. 1990). Considerando que cada electrodo contiene la señal de modo común junto al biopotencial de interés, al promediar se obtendrá una buena estimación de la tensión de modo común a la vez que el contenido asociado a los biopotenciales (que no forma parte de la señal a estimar) se anula entre sí. Sin embargo esta topología implica el riesgo de que la falla o desconexión de un único electrodo arruine la estimación y por lo tanto anule el funcionamiento del DRL. Esta forma de estimar $v_{\mathrm{CM}}$ suele ser utilizada únicamente en equipos pequeños con pocos canales de adquisición. En sistemas con un número de canales significativo, se utiliza un electrodo adicional, específico para obtener la tensión de modo común $v_{\mathrm{CM}}$.

\subsubsection{Diseño de $G_{\mathrm{DRL}}(s)$}

En la expresión (4-6) se observa que mientras mayor sea $G_{\mathrm{DRL}}$ a la frecuencia interferente, mayor será la reducción de la tensión de modo común. No obstante, partir de la Figura 4-2 puede apreciarse que el DRL funciona en un lazo cerrado en el que interviene no solo $G_{\mathrm{DRL}}(s)$, sino también funciones de transferencia conformadas por las impedancias de los electrodos, las impedancias de entrada del DRL y de los canales de medición, así como las capacidades parásitas del modelo EMI. Por consiguiente, al diseñar $G_{\mathrm{DRL}}(s)$, deberá tenerse en cuenta la estabilidad del sistema completo funcionando a lazo cerrado.

En la Figura 4-3 puede apreciarse una aplicación multicanal (con $\mathrm{N}$ electrodos conectados) donde $v_{\mathrm{CM}}^{\prime}$ es obtenida a partir de un electrodo independiente. Se supone que todos los electrodos de medida desarrollan la misma impedancia $Z_{\mathrm{EM}}$ y que cada canal presenta la misma capacidad de entrada de modo común $C_{\mathrm{IN}}$. 
La influencia de la tensión de red y las capacidades de acoplamiento con la misma son reemplazadas por su circuito equivalente de Thevenin.

$$
\begin{gathered}
V_{\mathrm{TH}}=V_{\mathrm{PL}} \cdot\left[\frac{C_{\mathrm{P}}}{C_{\mathrm{P}}+C_{\mathrm{B}}}-\frac{C_{\mathrm{SUP}}}{C_{\mathrm{SUP}}+C_{\mathrm{ISO}}}\right] \\
C_{\mathrm{TH}}=\left[\frac{1}{C_{\mathrm{P}}+C_{\mathrm{B}}}+\frac{1}{C_{\mathrm{SUP}}+C_{\mathrm{ISO}}}\right]^{-1}
\end{gathered}
$$

En la Figura 4-4 se describe el circuito anterior en un diagrama en bloques donde se aprecia claramente el lazo de control conformado por el DRL, que busca imponer una tensión de referencia $V_{\mathrm{REF}},{ }^{4}$ a la vez que debe rechazar el efecto de $V_{\mathrm{TH}}$ (o de $V_{\mathrm{PL}}$ ) la cual es vista como una perturbación a la salida del lazo.

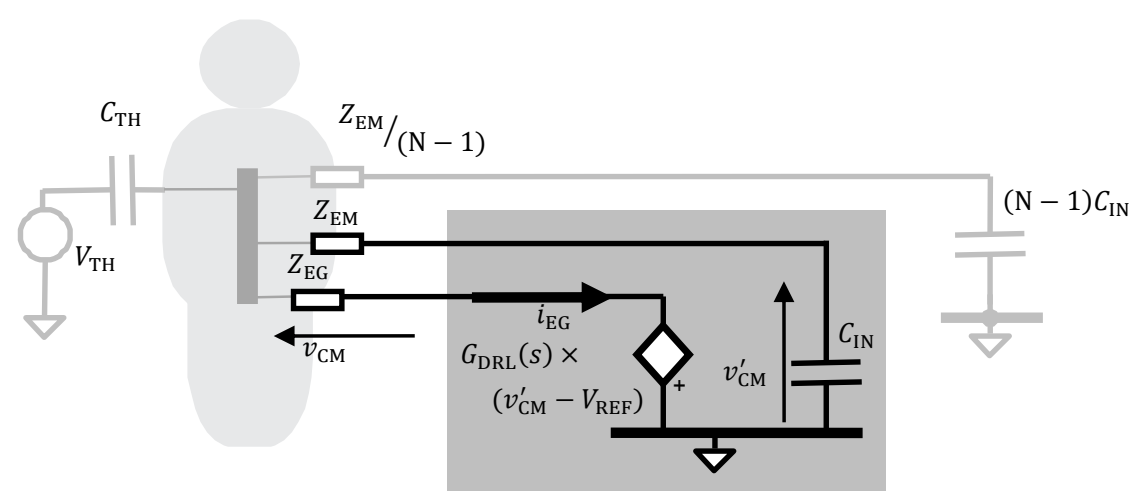

Figura 4-3: Modelo circuital utilizado para el análisis de la estabilidad, donde se considera una disposición con $\mathrm{N}$ electrodos, uno de los cuales se utiliza para estimar $v_{\mathrm{CM}}$ y el resto para la medición de los biopotenciales

\footnotetext{
${ }^{4}$ La tensión $V_{\text {REF }}$ puede ser el potencial de masa, o un valor fijo, para desplazar el nivel de continua (DC) del paciente respecto de las entradas del amplificador.
} 


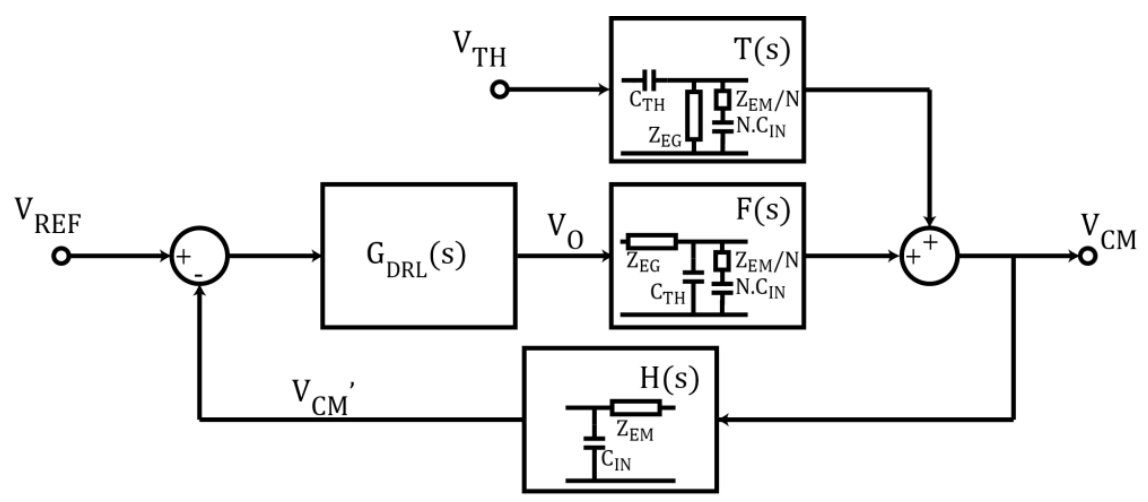

Figura 4-4: Diagrama en bloques que facilita la interpretación del DRL como lazo de control de la tensión de modo común. Las funciones de transferencia son independientes de cómo se mide $v_{\mathrm{CM}}$.

La estabilidad del sistema se logra asegurando márgenes de fase y ganancia adecuados para la transferencia de lazo abierto, que observando la Figura 4-4 puede expresarse como:

$$
\begin{aligned}
G_{\mathrm{OL}}(\mathrm{s})=G_{\mathrm{DRL}}(s) \cdot F(s) \cdot H(s)=\frac{G_{\mathrm{DRL}}(s)}{1+\frac{Z_{\mathrm{EM}}}{Z_{\mathrm{IN}}}+\frac{Z_{\mathrm{EG}}}{Z_{\mathrm{IN}}}\left(N+\frac{Z_{\mathrm{EM}}+Z_{\mathrm{IN}}}{Z_{\mathrm{TL}}}\right)} \\
=\frac{G_{\mathrm{DRL}}(s)}{1+s \cdot C_{\mathrm{IN}}\left(Z_{\mathrm{EM}}+N \cdot Z_{\mathrm{EG}}\right)+\mathrm{s} \cdot C_{\mathrm{TH}} \cdot Z_{\mathrm{EG}}+s^{2} \cdot C_{\mathrm{TH}} \cdot C_{\mathrm{IN}} \cdot Z_{\mathrm{EG}} \cdot Z_{\mathrm{EM}}}
\end{aligned}
$$

El diseño clásico para el DRL, propuesto por (Winter y Webster 1983) consiste en un integrador que ofrece una ganancia cercana a 30 veces a la frecuencia de red, lo cual implica $\tau_{\mathrm{I}}=100 \mu \mathrm{s}$ :

$$
\left.G_{\mathrm{DRL}}\right|_{\text {Clásico }}(s)=\frac{1}{\tau_{\mathrm{I}} \mathrm{s}}
$$

Dicha función de transferencia asegura una reducción de $v_{\mathrm{CM}}$ de 30 veces, equivalente a un incremento de $30 \mathrm{~dB}$ en el CMRR del sistema. Respecto a la estabilidad, esta solución adopta un criterio de diseño por polo dominante, donde se ha buscado tener una ganancia unitaria a $f_{\mathrm{C}} \approx 1,5 \mathrm{kHz}$ para que en la función de transferencia de lazo abierto domine la transferencia del integrador al menos hasta cruzar el eje de 0 dB. Según el análisis realizado por (Winter y Webster 1990a) dicha frecuencia es la más baja posible a la cual podría aparecer el primer polo de $F(s) . H(s)$, el cual aportaría $45^{\circ}$ de fase, si los siguientes polos estuviesen alejados al menos una década. Sumado a los $90^{\circ}$ de atraso de fase que aporta el integrador, el sistema queda con $45^{\circ}$ de margen de fase, con lo cual se asegura la estabilidad.

El caso analizado es muy pesimista ya que considera impedancias de electrodo $Z_{\mathrm{EM}}$ y $Z_{\mathrm{EG}}$ puramente resistivas (ignorando la capacidad en paralelo que "cortocircuita" el electrodo a frecuencias elevadas), así como valores demasiado altos para $C_{\mathrm{IN}}$, asociados a filtros de RF 0 al uso de cables blindados, que no son representativos en caso de utilizarse electrodos activos. Debido a esto, es muy común encontrar que en la práctica se implementa esta misma solución pero con $\tau_{\mathrm{I}}=33 \mu \mathrm{s}$, lo que implica una ganancia de 100 
veces $(40 \mathrm{~dB})$ a la frecuencia de red, incrementando $f_{\mathrm{C}}$ a aproximadamente $5 \mathrm{kHz}$, sin desestabilizar el sistema.

\subsection{3 ¿Cuánta ganancia del DRL es necesaria?}

Tomando como referencia los valores obtenidos experimentalmente para las capacidades del modelo EMI mostrados en el Capítulo 2, se observa que $v_{\mathrm{CM}}$, debida a la tensión de la red $V_{\mathrm{PL}}$, puede tomar valores de hasta $22 \mathrm{mV}_{\mathrm{RMS}}(60 \mathrm{mV}-\mathrm{p})$.

Teniendo en cuenta que el ruido del amplificador es de entre 2 y $3 \mu \mathrm{V}_{\mathrm{p}-\mathrm{p}}$, un $C M R R_{E} \approx 85 \mathrm{~dB}$ resultaría suficiente para que el efecto de $v_{\mathrm{CM}}$ no sea significativo. En general el efecto divisor de potencial, que puede ser menor de $60 \mathrm{~dB}$, domina frente al rechazo de modo común de un amplificador diferencial, y en este caso los 30dB de ganancia que aporta el DRL clásico serían suficientes. Elevar la ganancia del DRL por encima de estos $30 \mathrm{~dB}$, no sólo hace posible la operación con tensiones $v_{\mathrm{CM}}$ mayores de 20-30 mV sino que además permite utilizar amplificadores de bajo CMRR, por ejemplo amplificadores independientes en cada electrodo. Estos casos corresponden a electrodos preamplificados (Deguen y Jäckel 2004) y a electrodos capacitivos, donde diferencias entre las ganancias de los electrodos conducen a CMRR tan bajos como 30-40 dB (Haberman y Spinelli 2012) y se hace necesario incrementar la ganancia del DRL a la frecuencia de red a más de $60 \mathrm{~dB}$.

\subsection{Reducción de $v_{\mathrm{CM}}$ sintonizada}

Como se presentó en la sección previa, el diseño clásico de DRL utilizando un integrador tiene limitada la ganancia a la que puede llegar sin comprometer la estabilidad en unos $30 \mathrm{~dB}$ a $50 \mathrm{~Hz}$. Para incrementarla, se propuso un diseño alternativo que consiste en incluir un circuito sintonizado en la frecuencia de la interferencia a rechazar $\left(f_{\mathrm{PL}}=50 \mathrm{~Hz}\right)$, con alto factor de mérito $(\mathrm{Q})$. Este circuito funciona en paralelo con el integrador de la solución clásica (ver Figura 4-5), conformando la función de transferencia para el DRL expresada en (4-10).

$$
\left.G_{\mathrm{DRL}}\right|_{\text {sintonizado }}(s)=\frac{1}{\tau_{i} s}+K \frac{\left(2 \pi f_{\mathrm{PL}}\right)^{2}}{s^{2}+\left(\frac{2 \pi f_{\mathrm{PL}}}{Q}\right) s+\left(2 \pi f_{\mathrm{PL}}\right)^{2}}
$$

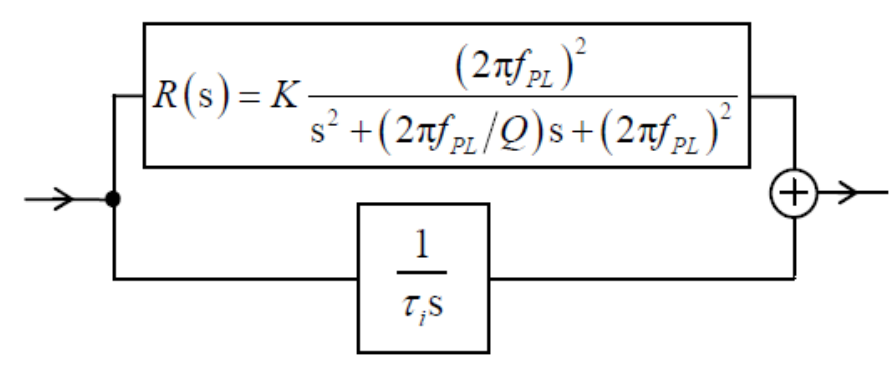

Figura 4-5: Diagrama en bloques de La función de transferencia del DRL propuesto 
La función de trasferencia del resonador $R(s)$ debe proporcionar una alta ganancia a la frecuencia de red mientras que su contribución cerca de la frecuencia de corte $f_{\mathrm{C}}$, debe ser despreciable comparada con la ganancia del integrador en dicha frecuencia $(0 \mathrm{~dB})$. De esta manera $G_{\mathrm{DRL}}(s)$ presenta una muy alta ganancia a $f_{\mathrm{PL}}$ mientras que a otras frecuencias se comporta como el integrador clásico (ver Figura 4-6). Esto último es el factor clave para mantener las condiciones de estabilidad del enfoque clásico, con un margen de fase de $45^{\circ}$. En resumen los requisitos de diseño para $R(s)$ son una muy alta ganancia en $f_{\mathrm{PL}}$ y una ganancia muy pequeña (menor a $-20 \mathrm{~dB}$ ) en $f_{\mathrm{C}}$ :

$$
\begin{gathered}
\left|R\left(j 2 \pi f_{\mathrm{PL}}\right)\right|=K Q \\
\left|R\left(j 2 \pi f_{\mathrm{C}}\right)\right| \approx K\left(\frac{f_{\mathrm{PL}}}{f_{\mathrm{C}}}\right)^{2} \ll 1
\end{gathered}
$$

La tensión $v_{\mathrm{CM}}$ será reducida por la ganancia a lazo abierto a $f_{\mathrm{PL}}$, esto es por $K Q$. Entonces, seleccionando un factor $Q$ lo suficientemente alto y una ganancia en continua $K$ apropiada, es posible dimensionar la ganancia pico y el ancho de banda $B W=\frac{2 \pi f_{\mathrm{PL}}}{Q}$.

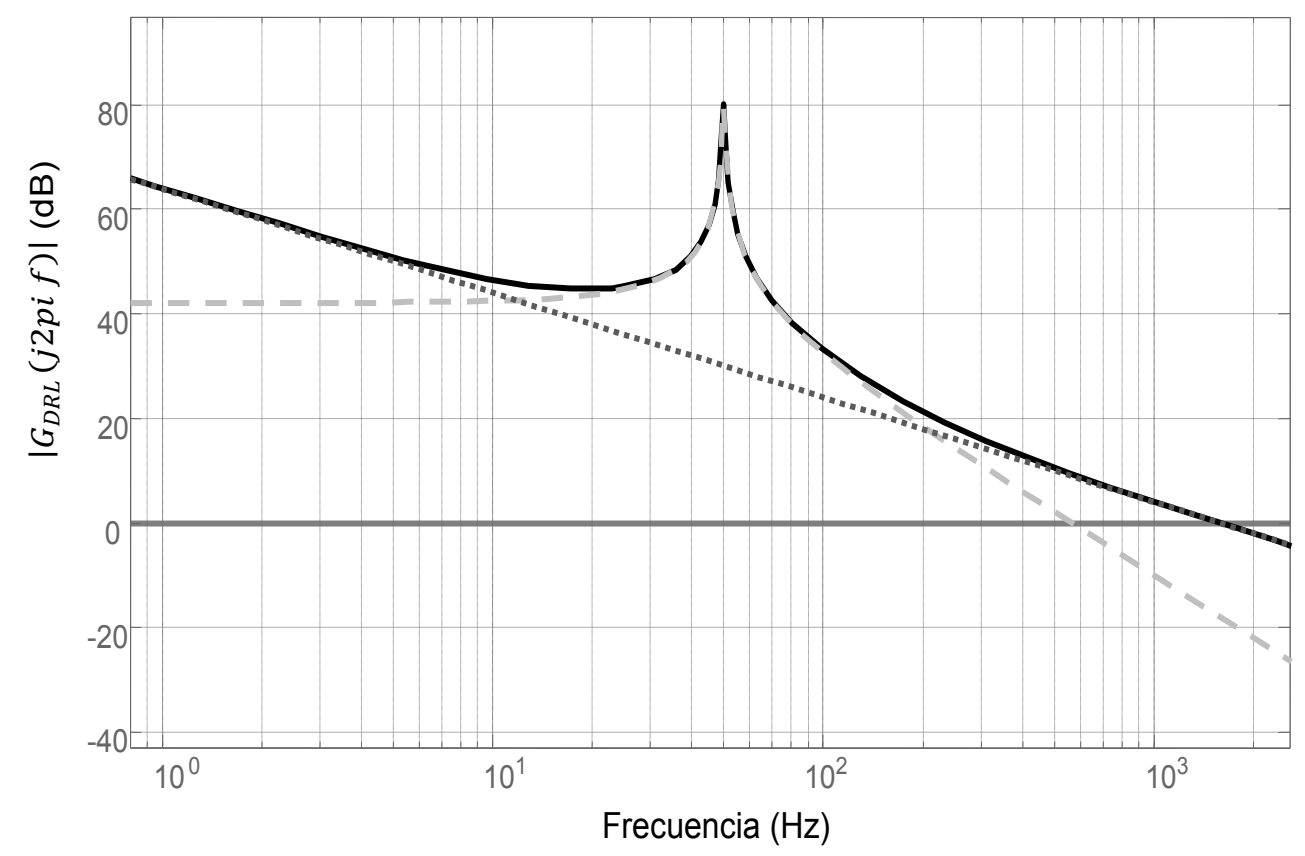

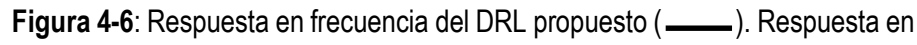
frecuencia del DRL integrador (Winter y Webster 1983a) (.......).Respuesta en frecuencia del circuito $R(s)$ sintonizado en $f_{\mathrm{PL}}=50 \mathrm{~Hz}$, con $K=127$ y $Q=$ $130(---)$.

La implementación del circuito propuesto requiere de un circuito de alto $Q$ con una frecuencia central muy precisa. Esto es algo difícil de lograr con circuitos analógicos teniendo en cuenta el envejecimiento, la tolerancia y la sensibilidad a la temperatura de los componentes electrónicos. Por esto se adoptó una implementación completamente digital para el $\mathrm{DRL}$, que provee una función de trasferencia precisa y estable en el tiempo. 
Al esquema propuesto podrían también incorporarse resonadores en armónicos de la red u otras frecuencias interferentes particulares. Nótese en la Figura 4-12 que el DDRL propuesto reduce -respecto del DRL clásico- la interferencia a $50 \mathrm{~Hz}$ pero no en $100 \mathrm{~Hz}$.

\subsubsection{Diseño e implementación del DRL Digital}

El esquema general propuesto para el circuito DRL Digital (DDRL) se muestra en la Figura 4-7, el cual está basado en un Controlador Digital de Señales (DSC) de bajo costo, el DSPIC33FJ128GP802 de Microchip Technology. El procesamiento digital se realizó en aritmética de punto fijo en 16 bits a una tasa de muestreo de $40 \mathrm{kHz}$. La tensión $v_{\mathrm{CM}}$ fue estimada por un electrodo independiente y digitalizada con un $A D C$ de 12 bits embebido en el DSC. Para hacer un buen uso del rango dinámico de este ADC, $v_{\mathrm{CM}}^{\prime}$ es amplificada 10 veces antes de ser digitalizada.

La función de transferencia $G_{\mathrm{DRL}}$ se diseñó utilizando un enfoque en el espacio de estados, que permite obtener una buena resolución en el posicionamiento de los polos para la limitada resolución de los coeficientes a utilizar (16 bits) (Porat 1997). El DCS realiza todo el procesamiento digital y transfiere la salida, a través de un canal SPI, a un conversor digital-analógico, el DAC8830 de Texas Instruments, que finalmente convierte la señal digital al mundo analógico.

La aritmética de punto fijo introduce restricciones en las posiciones de los polos (Porat 1997, Rabiner y Gold 1975), de forma tal que solo son posibles un número discreto de posiciones para los polos. Para la implementación del DDRL, se escogieron un par de polos complejos conjugados que producen una frecuencia central de $49.93 \mathrm{~Hz}$ y un factor $Q$ de $130(B W=0,4 \mathrm{~Hz})$. Adoptando $K=127$, se satisfacen las condiciones en (4-11), proveyendo una ganancia de $84 \mathrm{~dB}$ a $50 \mathrm{~Hz}$. Utilizando un DSP con unidad de punto flotante sería posible lograr una mejor resolución en el posicionamiento de los polos, pero esto implicaría mayor costo, complejidad y consumo de energía: un recurso escaso en equipos alimentados a baterías.

De todas formas, una reducción del orden de los $70 \mathrm{~dB}$ suele ser suficiente. Una inusual tensión de modo común de $200 \mathrm{mV}_{\mathrm{p}-\mathrm{p}}$, sería reducida a unos $60 \mu \mathrm{V}_{\mathrm{p}-\mathrm{p}}$. Incluso con un $C M R R_{\mathrm{E}}$ tan bajo como $30 \mathrm{~dB}$, la señal de modo diferencial producida por $v_{\mathrm{CM}}$ estaría por debajo del ruido de electrodo/amplificador, el cual es típicamente $2-3 \mu \mathrm{V}_{\mathrm{p}-\mathrm{p}}$.

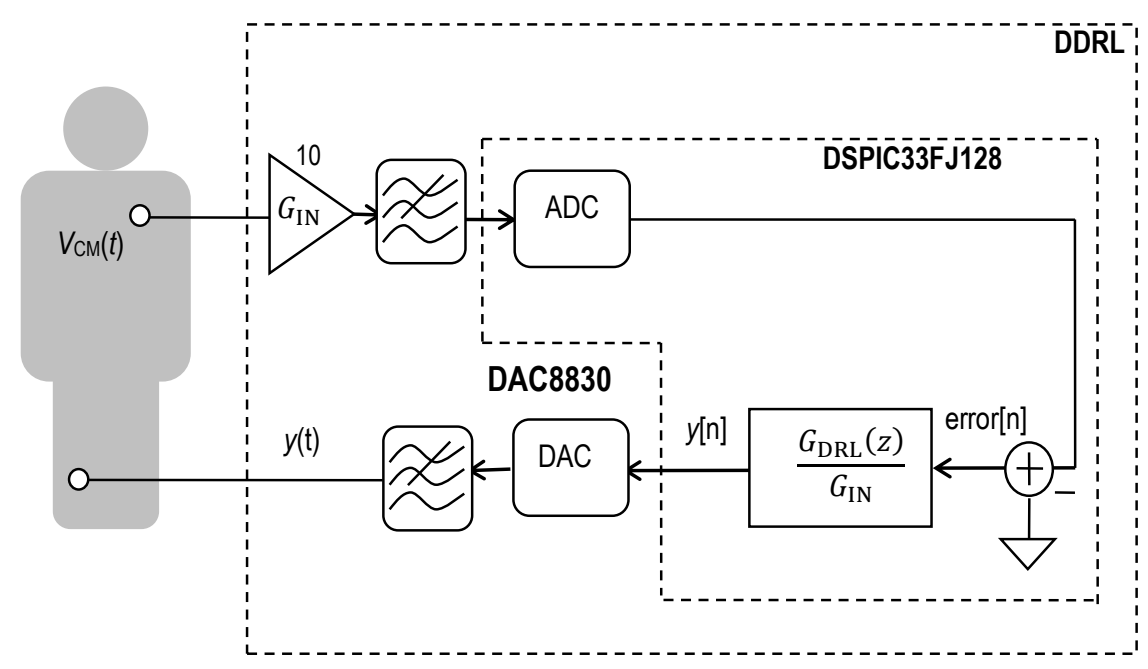

Figura 4-7: Diagrama en bloques del circuito que implementa el DDRL. 


\subsubsection{Resultados experimentales}

EI DDRL fue implementado y su función de transferencia fue medida en condiciones de laboratorio. Los datos experimentales resultantes se muestran en la Figura 4-8. El circuito muestra una notable ganancia de $74 \mathrm{~dB}$ a la frecuencia de red. La diferencia entre la ganancia experimental de $74 \mathrm{~dB}$ y la teórica de 80 $\mathrm{dB}$ se deben a la precisión limitada de las operaciones aritméticas, así como a la resolución del ADC.

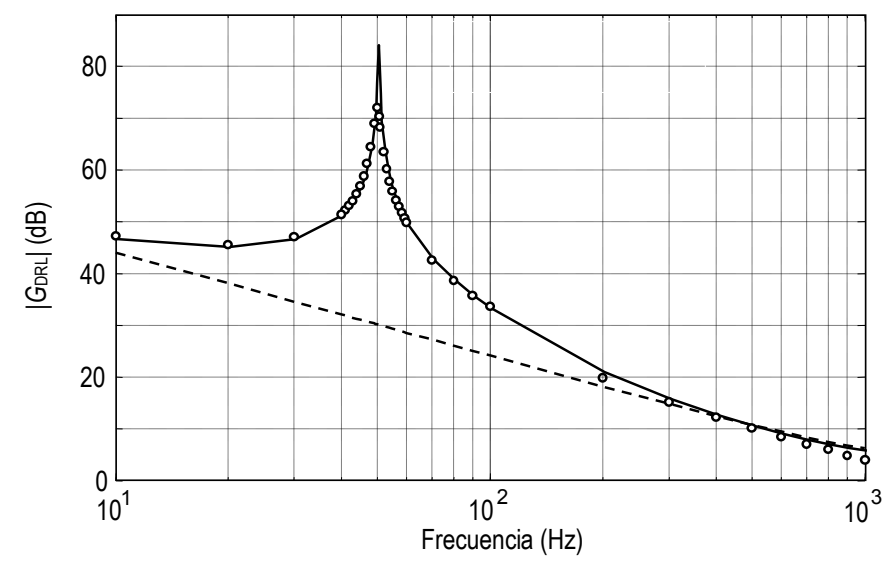

Figura 4-8: Respuesta en frecuencia del DDRL diseñado (—). Medidas experimentales de la respuesta en frecuencia del DDRL implementado (o). Respuesta en frecuencia del DRL integrador (_- - ).

\subsubsection{Esquema de adquisición multicanal basado en amplificadores Single- Ended y el DDRL}

Los bajos valores de $v_{\mathrm{CM}}$ logrados gracias a la muy alta reducción de que logra el circuito DDRL facilitan la implementación de sistemas de adquisición multicanales de biopotenciales con topologías simples, como arreglos de amplificadores single-ended desacoplados (Degen y Jäckel 2004, Levkov 1988). Pese a los conocidos pobres valores de CMRR que acarrean este tipo de topologías, la reducción directa de la tensión interferente es lo suficientemente alta para que sus efectos no sean apreciables.

Con el fin de probar el esquema propuesto, de múltiples canales single-ended operando en conjunto con el DDRL, fue montada la configuración experimental de la Figura 4-9 donde es adquirido un canal de ritmo alfa visual. El $A B$ está compuesto por dos preamplificadores single-ended no inversores como los utilizados en (Degen y Jäckel 2004), con ganancias desbalanceadas de 10.873 VN y 10.804 V/V respectivamente (esto implica un $C M R R_{\mathrm{E}}$ menor a $44 \mathrm{~dB}$, sin considerar el efecto divisor de potencial a la entrada del frontend). Los preamplificadores no inversores fueron construidos a partir de un Cl TLC2274 de Texas Instruments, que incluye cuatro operacionales de bajo ruido.

Los resultados se muestran en la Figura 4-10, donde no se observa ningún vestigio de interferencia de línea, en comparación a la utilización de un DRL con $30 \mathrm{~dB}$ de ganancia a $50 \mathrm{~Hz}$. Esto verifica la factibilidad del esquema propuesto.

En las Figura 4-11 y Figura 4-12, se repite la comparación anterior, pero forzando un deterioro de las condiciones de EMI, incorporando una resistencia de $100 \mathrm{~K} \Omega$ en serie con el electrodo de realimentación. 
La utilización de arreglo de amplificadores SE, tiene como objetivo la simplicidad de implementación de ABs multicanales de EEG y EMG, resultando en un circuito sencillo y de buen desempeño. El mismo consiste en un amplificador SE por canal, siendo apropiado para sistemas monopolares de gran número de canales y en electrodos preamplificados (Deguen y Jäckel 2004) o en cajas de conexión (headboxes) inalámbricas. El uso de amplificadores SE permite también un sencillo testeo de la calidad del contacto entre el electrodo y la piel (Levkov 1988), que sumado al control digital de la señal de modo común permite establecer variados esquemas para el rechazo de interferencias (Deguen y Jäckel 2004).

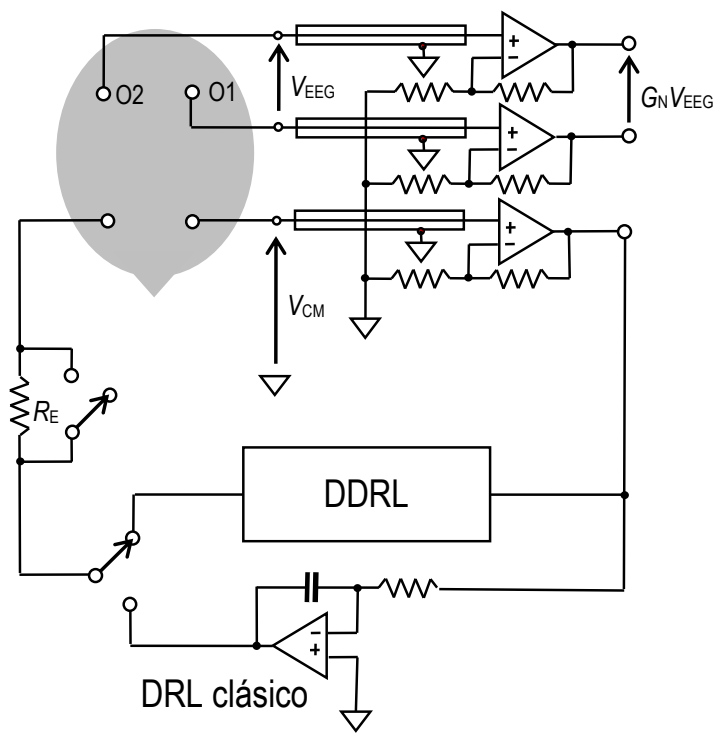

Figura 4-9: Configuración experimental para testear el esquema multicanal propuesto. Permite seleccionar entre el DDRL y el DRL clásico, así como intercalar un resistor en el lazo de realimentación a fin de simular un escenario de EMl adverso.

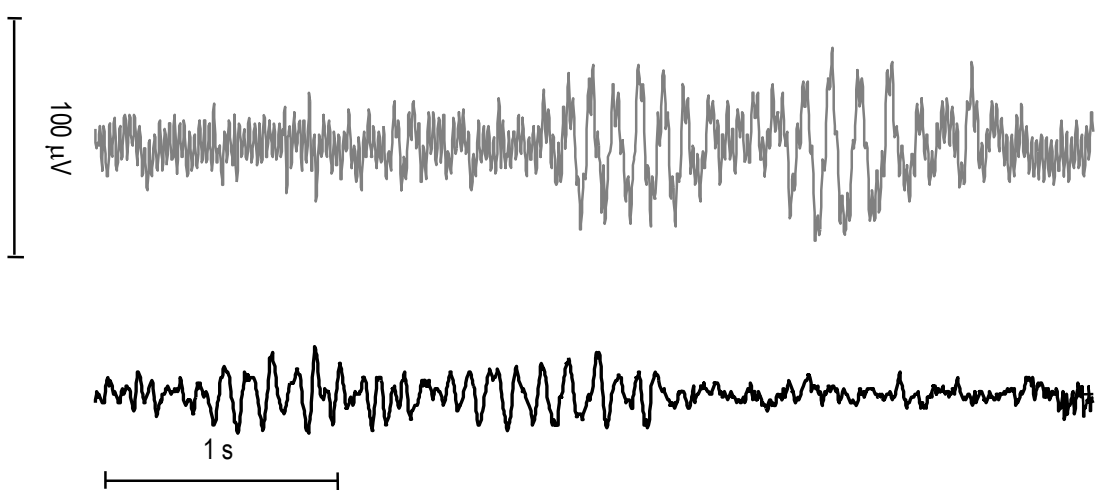

Figura 4-10: Señales de EEG adquiridas con el DRL clásico (arriba) y utilizando el DDRL (abajo). 


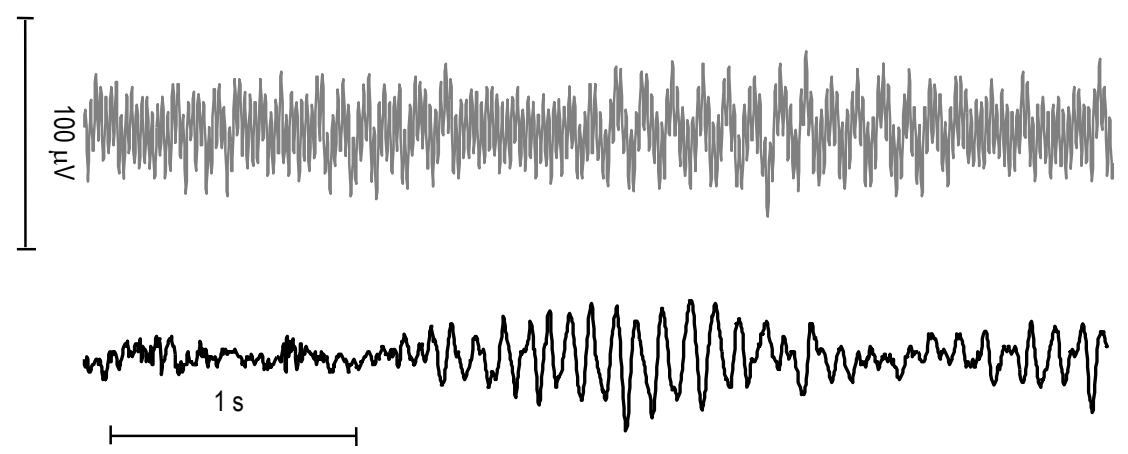

Figura 4-11: Señales de EEG adquiridas con el DRL clásico (arriba) y utilizando el DDRL (abajo). Para simular peores condiciones de interferencia se intercaló un resistor de $100 \mathrm{k} \Omega$ en serie con el electrodo de realimentación.

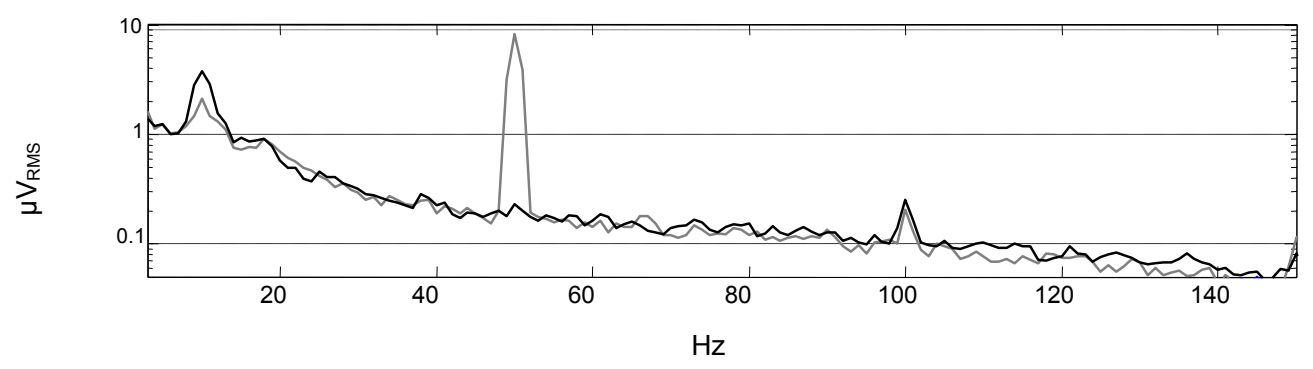

Figura 4-12: Espectro de las señales de EEG adquiridas usando el DRL clásico (curva gris) y el DDRL (curva negra). El resistor de $100 \mathrm{k} \Omega$ fue conectado en serie con el electrodo de realimentación para intensificar el efecto de la interferencia.

\subsection{Reducción de $v_{\mathrm{CM}}$ en sistemas capacitivos}

La reducción de $v_{\mathrm{CM}}$ mediante realimentación negativa es utilizada en configuraciones con electrodos capacitivos a partir de que el circuito Driven Seat Ground fuera publicado por Kim et al. (2005), (Aleksandrowicz y Leonhardt 2007, Baek et al. 2012, Chi et al. 2010b, Lim et al. 2010, Eilebrecht et al. 2010). El DRL capacitivo (CDRL) propuesto por Kim et al. con una ganancia de $40 \mathrm{~dB}$ logra una reducción de $v_{\mathrm{CM}}$ suficiente para adquirir señales de ECG sin saturar el amplificador, aunque aún necesita la utilización de un filtro notch a la frecuencia de red. La misma ganancia es usada por otros autores (Chi et al. 2010b, Lim et al. 2010), mientras que también son reportadas otras implementaciones de CDRLs con ganancias de hasta $60 \mathrm{~dB}$ (Aleksandrowicz y Leonhardt 2007). En general se observa que la literatura omite un tema importante: la estabilidad del lazo de realimentación de modo común.

Como será explicado en mayor detalle, algunos factores que no han sido tenidos en cuenta en los modelos más simplificados (Steffen et al. 2007) pueden contribuir a desestabilizar el lazo cerrado, limitando así la ganancia admisible:

- Elementos puramente resistivos en el lazo cerrado, como por ejemplo la impedancia de los tejidos (Chi et al. 2010a) y resistores de protección a la salida del amplificador de modo común (Chi et al. 2010b). 
- Aspectos más realistas en los modelos de los amplificadores operacionales, como sus polos en alta frecuencia que desestabilizan la respuesta en frecuencia para configuraciones de ganancia unitaria; así como el comportamiento con cargas capacitivas.

- Capacidades parásitas en ambientes no controlados (Haberman et al. 2011), que difieren de los "valores típicos" utilizados en la literatura (Kim et al. 2005).

En este sentido, se realizó un diseño racional de un CDRL, teniendo en cuenta los factores antes mencionados, a fin de lograr la eliminación de la interferencia de modo común para el peor de los casos, asegurando al mismo tiempo la estabilidad del sistema.

\subsubsection{Estimación de los posibles valores de $v_{\mathrm{CM}}$}

Para estimar los posibles valores de $v_{\mathrm{CM}}$ en un sistema capacitivo se utiliza el modelo EMI de la Figura 4-1, donde se consideran las impedancias de los electrodos $Z_{\mathrm{Ei}}$ como capacitivas. En particular considerando que la impedancia del electrodo de referencia $Z_{\mathrm{EG}}$ se modela con la capacidad $C_{\mathrm{GND}}$, la tensión $v_{\mathrm{CM}}$ queda descripta por (4-12).

$$
v_{\mathrm{CM}}=\frac{V_{\mathrm{PL}} \cdot\left|\frac{C_{\mathrm{P}}}{C_{\mathrm{P}}+C_{\mathrm{B}}}-\frac{C_{\mathrm{SUP}}}{C_{\mathrm{SUP}}+C_{\mathrm{ISO}}}\right|}{\frac{C_{\mathrm{GND}}}{C_{\mathrm{P}}+C_{\mathrm{B}}}+\frac{C_{\mathrm{GND}}}{C_{\mathrm{SUP}}+C_{\mathrm{ISO}}}+1}
$$

Como se observa en (4-12), el valor de $v_{\mathrm{CM}}$ depende de las capacidades parásitas $C_{\mathrm{P}}, C_{\mathrm{B}}, C_{\mathrm{ISo}}$ y $C_{\mathrm{SUP}}$, que son altamente variables y dependen de factores geométricos y demás condiciones del entorno. $A$ fin de estimar los posibles valores de $v_{\mathrm{CM}}$ en un sistema capacitivo se realizó una simulación de Monte Carlo donde se tomaron los rangos de variación para las capacidades parásitas indicados en la Tabla 4-1. Los mismos surgen de las medidas experimentales del Capítulo 2 y publicadas en (Haberman et al. 2011).

\begin{tabular}{ccc}
\hline Parámetro & Valor mínimo & Valor máximo \\
\hline $\boldsymbol{C}_{\mathbf{P}}$ & $50 \mathrm{fF}$ & $5 \mathrm{pF}$ \\
$\boldsymbol{C}_{\mathbf{B}}$ & $100 \mathrm{pF}$ & $4 \mathrm{nF}$ \\
$\boldsymbol{C}_{\text {SUP }}$ & $30 \mathrm{fF}$ & $3 \mathrm{pF}$ \\
$\boldsymbol{C}_{\text {ISO }}$ & $15 \mathrm{pF}$ & $200 \mathrm{pF}$ \\
\hline
\end{tabular}

Tabla 4-1: Valores de las capacidades parásitas utilizados en la simulación de Monte Carlo.

La simulación fue realizada considerando $C_{\mathrm{GND}}=1 \mathrm{nF}$ (un electrodo de referencia de tamaño moderado $\approx 25 \mathrm{~cm}^{2}$ ) y el resultado de 50.000 corridas fue que el rango de variación de la tensión $v_{\mathrm{CM}}$ es de $3 \mathrm{mV}$ a $700 \mathrm{mV}$, con una media de $250 \mathrm{mV}$ y una desviación estándar de aproximadamente $150 \mathrm{mV}$. 
Para tener en consideración cuán perjudiciales pueden ser estos valores, se analiza el efecto divisor de potencial en un sistema con electrodos capacitivos (ver Figura 4-13), dado que en cada electrodo se forma un divisor de tensión capacitivo entre la capacidad del electrodo $C_{\mathrm{S}}$ y la capacidad de entrada del seguidor de tensión $C_{\text {in }}$. Como fue mencionado en el Capítulo 2, los desbalances entre las capacidades de ambos electrodos provocan la aparición de una tensión diferencial $v_{\mathrm{DM}}$ a partir de $v_{\mathrm{CM}}$ :

$$
v_{\mathrm{DM}}=v_{\mathrm{CM}}\left(\frac{C_{\mathrm{in} 1}}{C_{\mathrm{in} 1}+C_{\mathrm{S} 1}}-\frac{C_{\mathrm{in} 2}}{C_{\mathrm{in} 2}+C_{\mathrm{S} 2}}\right)
$$

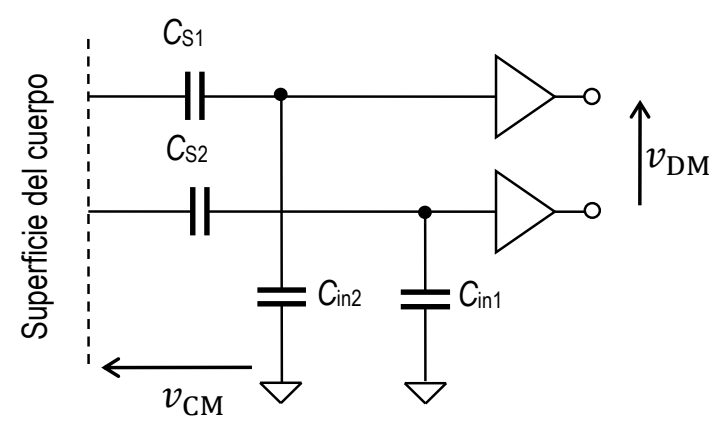

Figura 4-13: Circuito equivalente a la entrada de dos electrodos capacitivos que ilustra la transformación de modo debido al efecto divisor de potencial.

A partir de lo cual, considerando capacidades de entrada similares $\left(C_{\mathrm{in} 1} \approx C_{\mathrm{in} 2} \approx C_{\mathrm{in}}\right)$ y al menos un orden menor que las de los electrodos, el CMRR de un sistema capacitivo resulta:

$$
C M R R=\frac{C_{\mathrm{S} 1} C_{\mathrm{S} 2}}{C_{\mathrm{in} \cdot} \cdot C_{\mathrm{S} 1}-C_{\mathrm{S} 2} \mid}
$$

En una aplicación típica, los electrodos de medida tendrán neutralizadas sus capacidades de entrada $C_{\text {in }}$ mediante realimentación positiva (Spinelli y Haberman 2010) a unas pocas décimas de picofaradio (0.1 $0.2 \mathrm{pF}$ ). Mientras que las capacidades de acoplamiento de los electrodos $C_{\mathrm{Si}}$ tomarán valores que van de decenas de picofaradio $(10-30 \mathrm{pF})$ cuando se apliquen sobre una prenda de algodón, a cientos de picofaradios $(100-300 \mathrm{pF})$ cuando se apliquen sobre la piel (Chi et al. 2010a). Por ejemplo, considerando $C_{\mathrm{in}}=0.2 \mathrm{pF}, C_{\mathrm{S} 1}=10 \mathrm{pF}$ y $C_{\mathrm{S} 2}=20 \mathrm{pF}$ (valores típicos para electrodos sobre una prenda de algodón) la ecuación (4-14) da un CMRR de 100 veces (40 dB) el cual es un muy pobre.

Un valor de $v_{\mathrm{CM}}$ de $700 \mathrm{mV}$ con un CMRR de $40 \mathrm{~dB}$ daría como resultado la aparición de una señal diferencial de $7 \mathrm{mV}$, lo cual sería demasiado alto hasta para la adquisición de ECG. Para reducir dicha interferencia a un nivel de $7 \mu \mathrm{V}$ serían necesarios $60 \mathrm{~dB}$ adicionales de rechazo o reducción de $v_{\mathrm{CM}}$, haciéndola aceptable para la medición de ECG, pero para aplicaciones de EEG y EMG se requieren más de $80 \mathrm{~dB}$ de reducción de $v_{\mathrm{CM}}$. 


\subsubsection{Modelo del DRL capacitivo}

El esquema general del DRL Capacitivo (CDRL) es presentado en la Figura 4-14 (a), donde $v_{\mathrm{CM}}$ es estimado a partir de la medida del electrodo capacitivo $C_{\mathrm{E}}$, amplificado y realimentado a través del electrodo $C_{\mathrm{GND}}$. El circuito equivalente usualmente utilizado para el análisis del CDRL se observa en la Figura 4-14 (b). De acuerdo a este esquema, el sistema admite una $G_{\mathrm{DRL}}$ tan alta como sea necesaria, pero desafortunadamente esto no es cierto en la práctica dado que el sistema se vuelve inestable cuando la ganancia aumenta demasiado. Este comportamiento no puede ser explicado con el modelo simplificado de la Figura 4-14 (b). Para determinar las condiciones que llevan al sistema a la inestabilidad y poder diseñar un CDRL estable con la ganancia necesaria, se debe formular un modelo más completo, teniendo en cuenta la impedancia resistiva del cuerpo y otras componentes resistivas en serie con los electrodos, así como la respuesta en frecuencia del seguidor de tensión del electrodo de medida. Dicho modelo se muestra en la Figura 4-15.

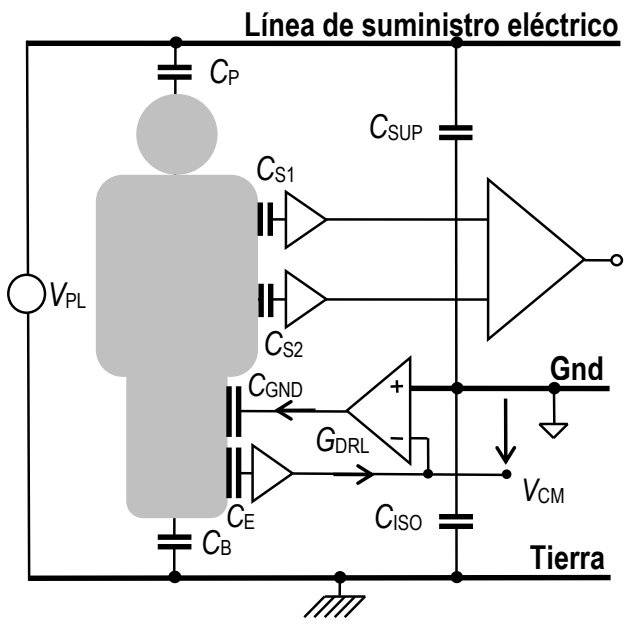

(a)

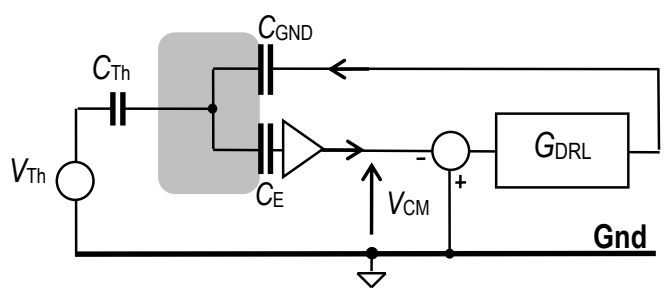

(b)

Figura 4-14: (a) Esquema general del circuito DRL Capacitivo (CDRL). (b) circuito equivalente simplificado para el análisis de la estabilidad.

Un problema adicional ocurre debido a las perturbaciones de baja frecuencia como las corrientes de polarización y las tensiones de offset, que limitan la tensión admisible máxima en DC a fin de prevenir la saturación del amplificador. La función de transferencia del CDRL, $G_{\mathrm{DRL}}(s)$, deberá diseñarse para asegurar la estabilidad del sistema y estar acoplada en AC para prevenir la saturación dadas las perturbaciones de baja frecuencia. 


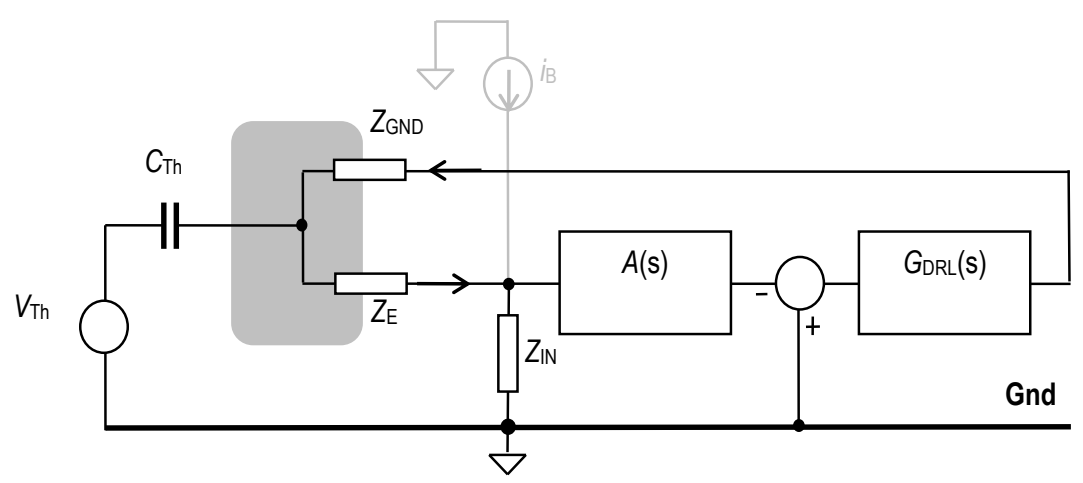

Figura 4-15: Un modelo de CDRL para el análisis de la estabilidad que incluye la respuesta en frecuencia del seguidor de tensión del electrodo de medida y modelos de impedancia de electrodos con una componente resistiva serie representando la impedancia del cuerpo. Se indica en gris la corriente de polarización del amplificador $i_{\mathrm{B}}$ que demanda una transferencia $G_{\mathrm{DRL}}(s)$ acoplada en AC.

\subsubsection{Circuito propuesto}

El circuito propuesto consiste en dos electrodos capacitivos de tamaño moderado $\left(25 \mathrm{~cm}^{2}\right)$. Uno de ellos mide $v_{\mathrm{CM}}$ mientras que el otro actúa manejando el potencial de modo común del sujeto. Para el diseño de la transferencia $G_{\mathrm{DRL}}(s)$ se debe tener en cuenta la estabilidad del lazo, considerando para este análisis diversos parámetros como las capacidades parásitas $C_{\mathrm{P}}, C_{\mathrm{B}}, C_{\mathrm{ISO}}$ y $C_{\mathrm{SUP}}$, las impedancias de electrodo $Z_{\mathrm{E}}$ y $Z_{\mathrm{GND}}$, la función de transferencia del seguidor de tensión $A(s)$ y su capacidad de entrada $C_{\mathrm{IN}}$. El modelo de impedancia de electrodo adoptado consiste en la capacidad $C_{\mathrm{Ei}}$ en serie con una resistencia $R_{\mathrm{Si}}$ que contempla la impedancia del tejido (Chi et al. 2010a). Esto lleva a un modelo bastante más realista pero demasiado complejo para hallar el caso más desfavorable, como se ha hecho para diseño del DRL clásico (Winter y Webster 1983).

Para hallar la situación potencialmente más inestable, se estimaron las posibles ubicaciones de los polos y la consiguiente respuesta en frecuencia a lazo abierto mediante otra simulación por el método de Monte Carlo para los rangos de variación de los parámetros mostrados en la Tabla 4-2. La respuesta en frecuencia del seguidor de tensión fue extraída de una simulación de barrido en frecuencia utilizando el modelo PSpice de Texas Instruments para el OPA320. La capacidad de entrada $C_{\mathrm{IN}}$ se fijó en $10 \mathrm{pF}$, dado que en el diseño propuesto no se pretende utilizar neutralización, esta capacidad contempla la capacidad de entrada del seguidor de tensión así como capacidades parásitas de la placa de circuito impreso.

Como se observa en la Figura 4-16, la posición más baja de un polo ocurre por encima de los $800 \mathrm{kHz}$, mientras que la ganancia es siempre menor que $0 \mathrm{~dB}$ para cualquier combinación de los parámetros (a excepción de un pico producido por la respuesta en frecuencia del seguidor de tensión en torno a las decenas de $\mathrm{MHz}$, que será atenuado por la misma respuesta del DRL). Por consiguiente, si se diseña $G_{\mathrm{DRL}}(s)$ como un pasabajos de primer orden, cuya ganancia descienda a $0 \mathrm{~dB}$ a una frecuencia menor a $800 \mathrm{kHz}$, el sistema será estable para cualquier combinación de los parámetros involucrados, de acuerdo a los rangos enumerados en la Tabla 4-2. 


\begin{tabular}{ccc}
\hline Parámetro & Valor mínimo & Valor máximo \\
\hline $\begin{array}{c}\boldsymbol{C}_{\mathrm{P}}, \boldsymbol{C}_{\mathrm{B}}, \\
\boldsymbol{C}_{\mathrm{SUP}}, \boldsymbol{C}_{\mathrm{ISO}}\end{array}$ & Ver Tabla 4-1 & Ver Tabla 4-1 \\
$\boldsymbol{R}_{\mathbf{S i}}$ & $500 \Omega$ & $1500 \Omega$ \\
$\boldsymbol{C}_{\mathrm{Ei}}$ & $30 \mathrm{pF}$ & $1 \mathrm{nF}$ \\
$\boldsymbol{C}_{\mathrm{in}}$ & $10 \mathrm{pF}$ & $10 \mathrm{pF}$ \\
\hline
\end{tabular}

Tabla 4-2: Rangos de variación de los valores utilizados en la simulación de Monte Carlo. Extraídos de Haberman et al. (2011) y Chi et al. (2010a)

La función de transferencia implementada para $G_{\mathrm{DRL}}(s)$, incluyendo el acoplamiento en alterna, con un polo en $16 \mathrm{~Hz}$, es la que se muestra en (4-15). En la Figura 4-17 se muestra el circuito que implementa la $G_{\mathrm{DRL}}(s)$, junto con el esquema completo del CDRL.

$$
G_{\mathrm{DRL}}(s)=\frac{-\left(\frac{R_{\mathrm{F}}}{R_{\mathrm{IN}}}\right) s}{\left(s+\frac{1}{R_{\mathrm{IN}} C_{2}}\right)\left(1+s \cdot R_{\mathrm{F}} C_{1}\right)}=\frac{-4700 . s}{(s+2 \pi 16 \mathrm{~Hz})\left(1+\frac{s}{2 \pi 103 \mathrm{~Hz}}\right)}
$$

EI CDRL fue construido como un bloque independiente que incluye los electrodos capacitivos de medida y de realimentación (ver Figura 4-18). El mismo puede utilizarse como un brazalete 0 una banda pectoral. Los electrodos están construidos por cinta de cobre 3M "Scotch Electrical Tape" colocados sobre un sustrato dieléctrico flexible de goma EVA (Etilvinilacetato) de $2 \mathrm{~mm}$. El circuito del CDRL mostrado en la Figura 4-17 cuenta con una guarda activa en el electrodo de medida, no obstante no se utilizó ningún circuito de neutralización de la capacidad de entrada dado que la capacidad del electrodo $C_{\mathrm{E}}$ es lo suficientemente grande para despreciar el efecto de la capacidad de entrada del seguidor de tensión. Cada electrodo, aislado por un film de polipropileno $\left(\epsilon_{\mathrm{R}} \approx 2,5\right)$ de $50 \mu \mathrm{m}$, presenta una capacitancia de aproximadamente $1 \mathrm{nF}$ cuando se lo coloca directamente en contacto con el cuerpo. Cuando el CDRL es utilizado sobre una prenda de algodón (de aproximadamente $350 \mu \mathrm{m}$ de espesor) la capacitancia de cada electrodo se reduce alrededor de 20 veces (Spinelli y Haberman 2010), (Chi et al, 2010a). 
Capítulo 4 - Reducción de interferencia de modo común

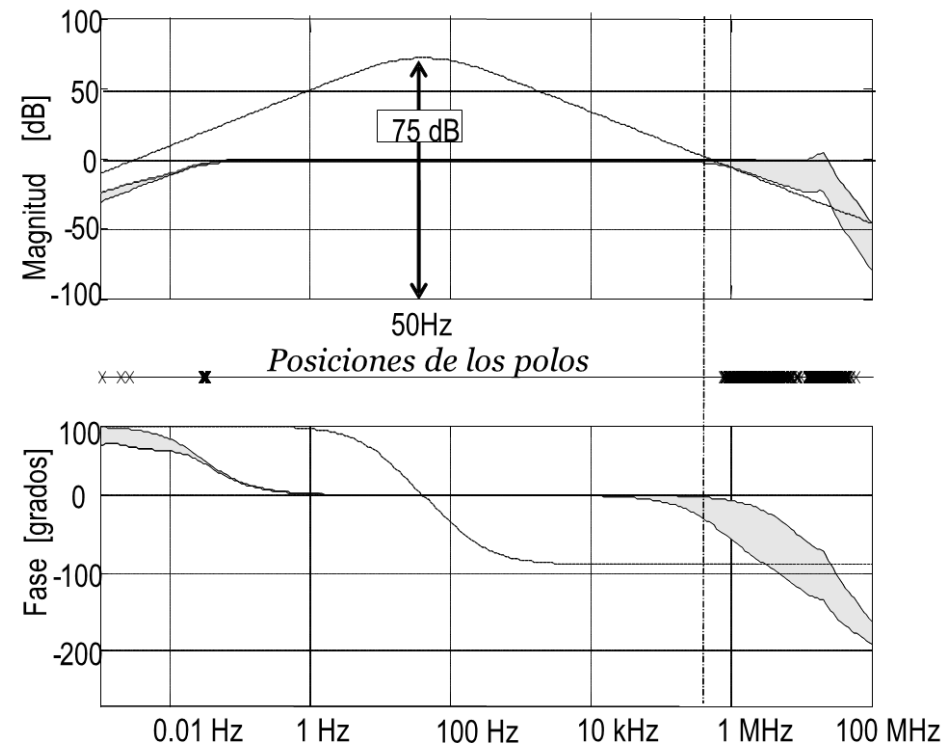

Figura 4-16: Área gris: respuestas de lazo abierto obtenidas por simulación de Monte Carlo con el conjunto de parámetros de las Tabla 4-1 y Tabla 4-2. Con cruces se indican las posibles posiciones de los polos de lazo abierto. En línea punteada se muestra la respuesta en frecuencia $G_{\mathrm{DRL}}(\mathrm{s})$ del CDRL propuesto.

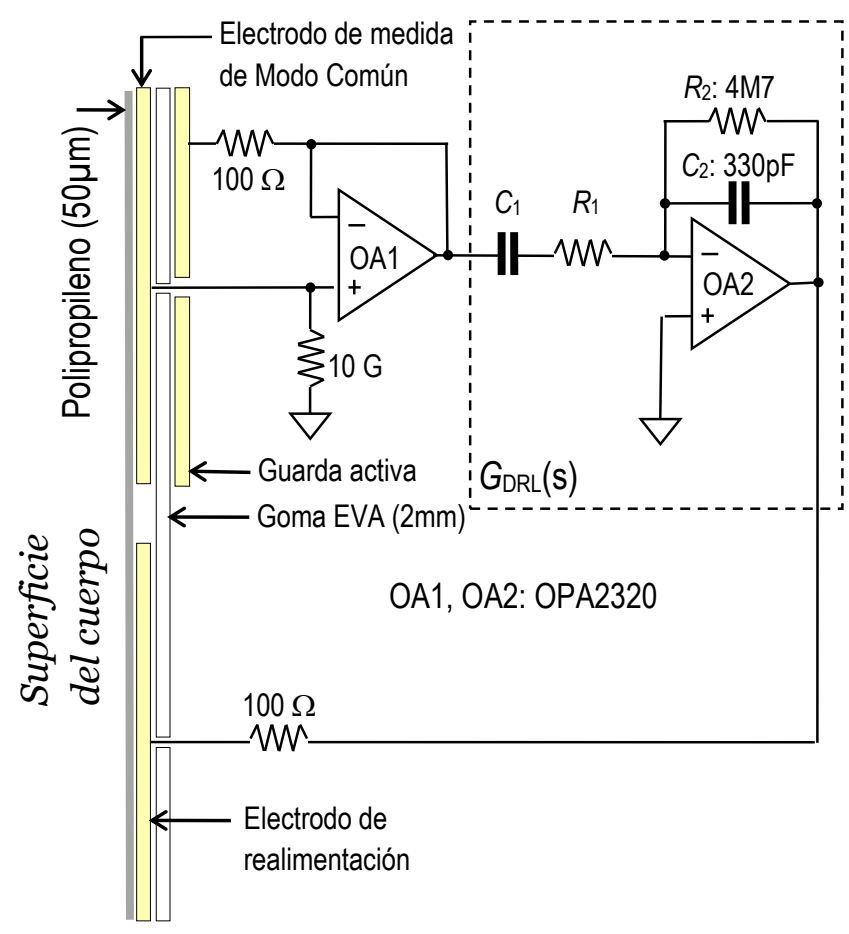

Figura 4-17: Esquema con el circuito completo que implementa el CDRL propuesto 


\subsubsection{Resultados experimentales}

A fin de observar el desempeño del circuito implementado para el CDRL, se adquirieron dos canales SE de ECG de acuerdo al esquema mostrado en la Figura 4-19 mediante dos electrodos capacitivos (Spinelli y Haberman 2010) montados en el pecho de un voluntario sobre una remera de algodón. El CDRL fue colocado en su espalda, también sobre la prenda de algodón. El sujeto se ubicó dentro de un entorno normal de oficina, al lado de un escritorio con una computadora, un monitor y otro equipamiento electrónico en funcionamiento. Todos los electrodos fueron sujetados mediante una cinta pectoral de velcro y los canales SE fueron digitalizados por un ADC Sigma Delta de 24 bits (ADS1298 de Texas Instruments) a una tasa de 500 muestras por segundo. La señal diferencial entre los electrodos (derivación I del ECG) fue obtenida digitalmente mediante sustracción de las señales SE. La configuración utilizada corresponde a un sistema completamente capacitivo donde no se utilizó ningún electrodo húmedo o seco.

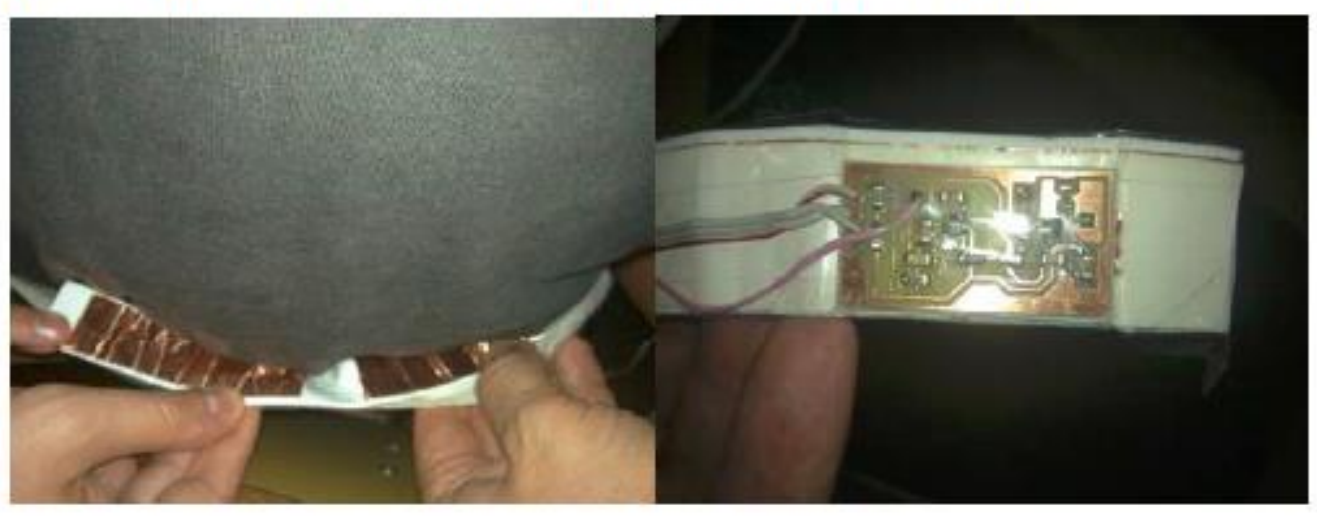

Figura 4-18: Prototipo implementado del CDRL, utilizado para obtener medidas experimentales de ECG

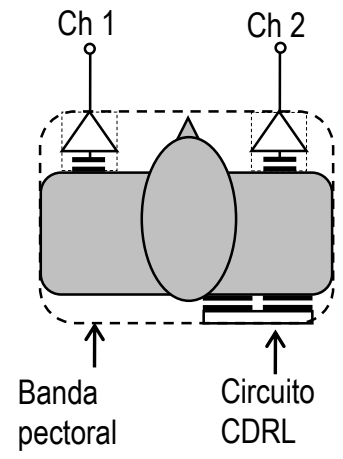

Figura 4-19: Configuración experimental con dos electrodos capacitivos en el pecho del voluntario y el CDRL en su espalda. Todos montados sobre una prenda de algodón. 
En primer lugar se obtuvieron las señales de ECG sin activar el CDRL, utilizando una gran capacidad $C_{\mathrm{GND}} \approx 10 \mathrm{nF}$ (cubriendo la silla con cinta de cobre $3 \mathrm{M}$ y formando grandes áreas conectadas a masa). En la Figura 4-20 pueden observarse las señales SE adquiridas, donde hay un gran nivel de interferencia de $50 \mathrm{~Hz}$ (hasta $10 \mathrm{mV}_{\mathrm{PP}}$ ) y también en la señal diferencial, dado el pobre CMRR inherente a las configuraciones capacitivas.

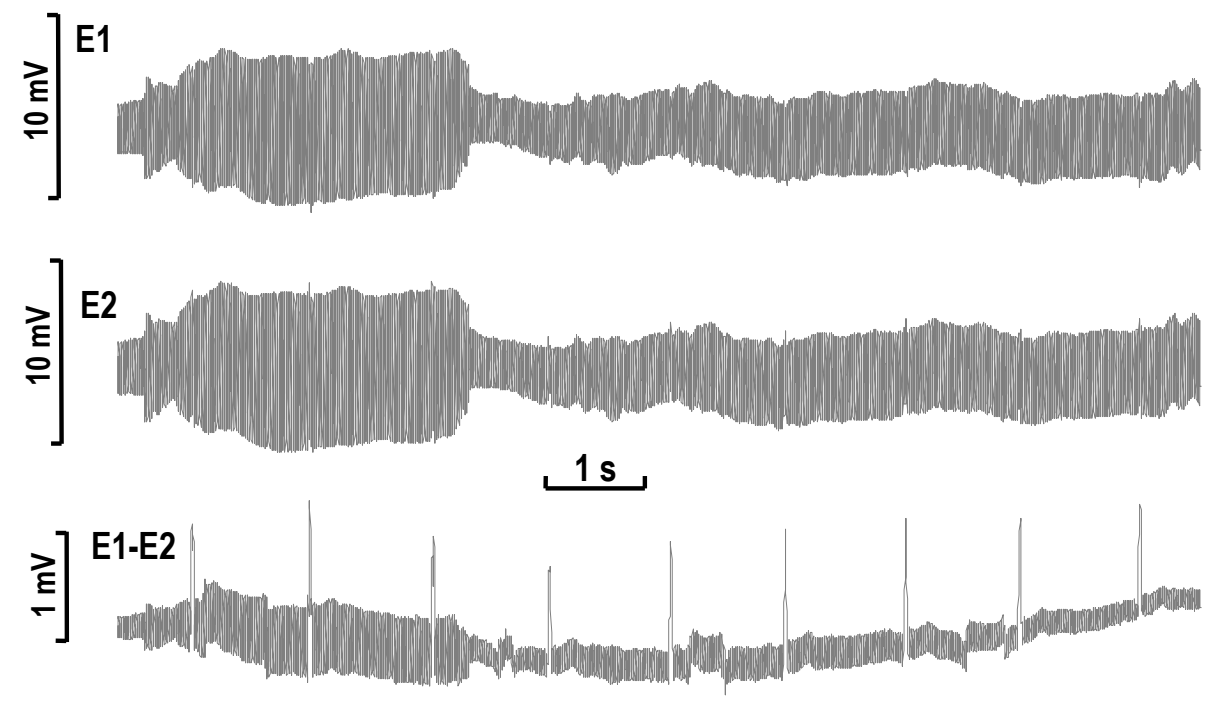

Figura 4-20: Señales de ECG adquiridas utilizando canales SE y una gran capacidad $C_{\mathrm{GND}} \approx 10 \mathrm{nF}$ en lugar del CDRL. Los trazos superiores corresponden a los canales SE y el inferior a su diferencia.

Con la misma disposición se activó el CDRL y los resultados se muestran en la Figura 4-21, donde se puede observar la baja interferencia en los canales SE que es reducida aún más al calcular la derivación diferencial. Nótese que también los canales SE llevan una clara y distinguible información de la respiración. 


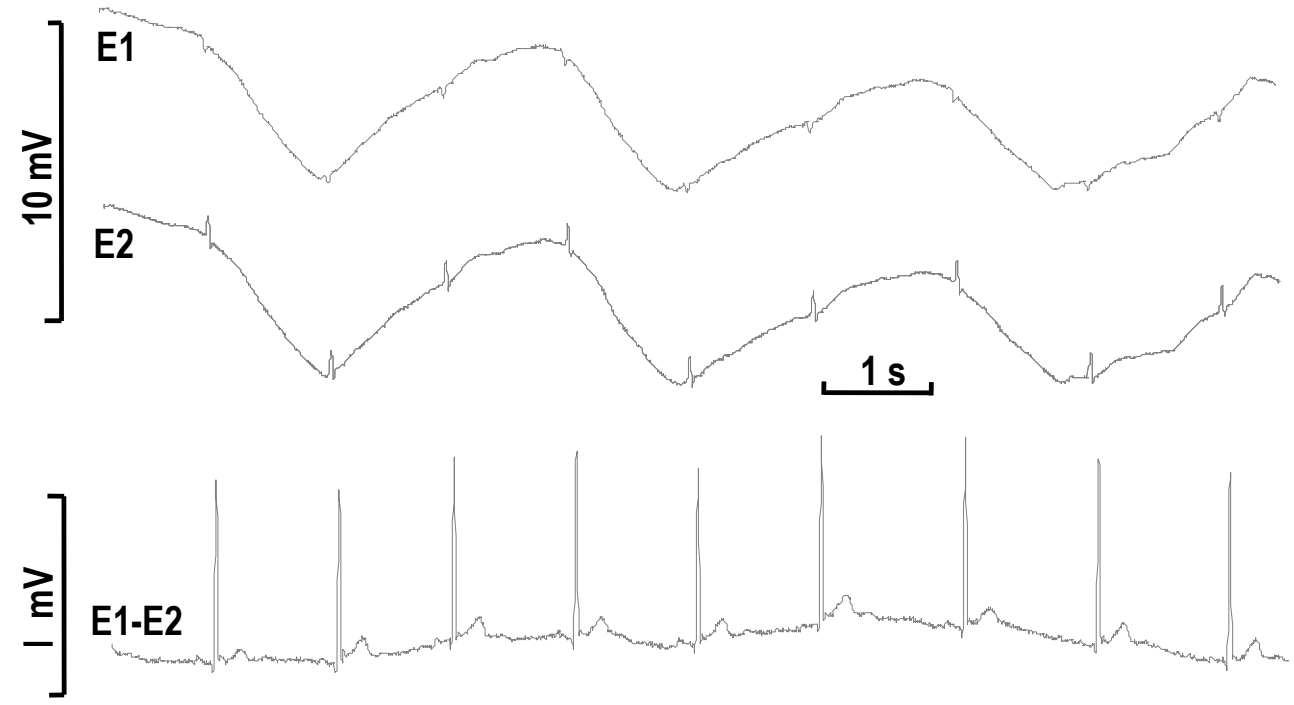

Figura 4-21: Señales de ECG adquiridas utilizando canales SE y el CDRL. LoS trazos superiores corresponden a los canales SE y el inferior a su diferencia.

Los electrodos también fueron evaluados en medidas de EMG sobre el antebrazo. En la Figura 4-22 se observa la señal diferencial libre de interferencia, donde claramente pueden observarse las contracciones producidas al flexionar los dedos. Esto muestra la aptitud del esquema propuesto para su utilización en un DA controlado por señales de EMG, adquiridas de forma completamente aislada.

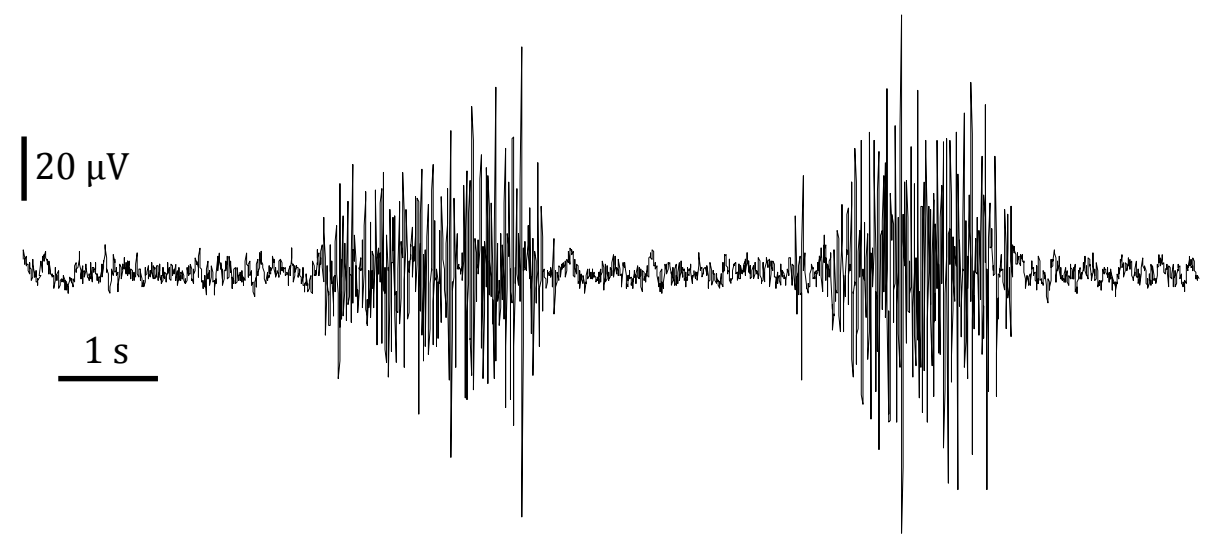

Figura 4-22: Señal de EMG adquirida sobre el antebrazo, utilizando electrodos capacitivos y el CDRL diseñado. Se observa el incremento de amplitud de la señal al contraer los dedos de la mano. 


\subsubsection{Discusión}

La utilización de electrodos de gran área para los CDRL, combinados con ganancias menores a $60 \mathrm{~dB}$, así como una favorable distribución de capacidades parásitas en ambientes de laboratorio no representan una gran amenaza a la estabilidad de los sistemas capacitivos. Esta es una razón más que entendible por la cual se suele evitar el análisis de estabilidad en la literatura: los circuitos funcionan correctamente, reduciendo $v_{\mathrm{CM}}$ sin desestabilizarse.

Sin embargo, persiguiendo el objetivo de desarrollar DAs que operen en ambientes no controlados es necesario un análisis basado en un modelo más realista para entender los límites de la estabilidad. La pérdida de la simplicidad en el análisis que acarrea el uso de un modelo más complejo requirió de técnicas de simulación estadística como el método de Monte Carlo.

\subsection{Sensor activo de EMG con reducción de modo común in-situ}

Tal como se vio en el Capítulo 3, tras varios diseños de electrodos activos para EMG, se desarrolló un sensor que incluye los electrodos secos, junto con todo el acondicionamiento de señal y procesamiento analógico, e incorpora también la realimentación de modo común. El mismo aprovecha la característica diferencial de la señal de EMG y la cercanía entre los puntos de medida para integrar todo en un mismo soporte.

El sensor está implementado en una placa de circuito impreso que consta de tres electrodos de acero inoxidable (ver Figura 3-5) que pueden servir como electrodos secos o como soporte para electrodos húmedos. Dos electrodos activos son los que adquieren la señal diferencial de EMG y están conectados directamente a un amplificador de instrumentación completamente diferencial, mientras que un tercero realimenta al sujeto la salida del DRL implementado en la misma placa, reduciendo $v_{\mathrm{CM}}$ e imponiendo al usuario la tensión de referencia $V_{\mathrm{REF}}$.

El valor estimado de la tensión de modo común $v_{\mathrm{CM}}^{\prime}$, es calculado como el promedio de ambas salidas diferenciales (el amplificador tiene ganancia de modo común unitaria, $G_{C C}=1$ ) y la diferencia con la tensión de referencia se realimenta a través de la función de transferencia del DRL $G_{\mathrm{DRL}}(s)$.

Si bien se ha analizado que una ganancia de $40 \mathrm{~dB}$ a la frecuencia de red es suficiente aun en condiciones adversas, es deseable también rechazar interferencia de modo común que puede acoplarse en otras frecuencias más altas, como los armónicos de la frecuencia de red o frecuencias del orden del kHz, donde suele estar concentrada la energía interferente de lámparas fluorescentes (Winter y Webster 1983,Spinelli et al. 1999). Esta energía, si bien queda por fuera del ancho de banda de interés, puede provocar comportamientos no lineales en el $A B$ o replicado en las señales digitalizadas. Por este motivo, y para aprovechar la ventaja de tener bajas capacidades de entrada $C_{\mathrm{IN}}$, se buscó resolver la ubicación del polo más bajo posible de $H(s) . F(s)$, a fin de mejorar el diseño de $G_{\mathrm{DRL}}(s)$.

A diferencia del enfoque llevado a cabo por Winter y Webster (1983), no se consideraron impedancias de electrodo puramente resistivas, ni se utilizaron filtros de RF en las entradas de los amplificadores. Para las impedancias de electrodos se adoptó un modelo como el de la Figura 3-1, el mismo adoptado por (Levkov1988, Chi et al. 2010a), que tiene en cuenta el efecto de la capacitancia que cortocircuita la componente resistiva a altas frecuencias. Para la capacidad de entrada de modo común que ve cada electrodo se consideraron $10 \mathrm{pF}$, los cuales incluyen los $8 \mathrm{pF}$ propios de las entradas del TLC2274 utilizado para implementar el electrodo activo. 


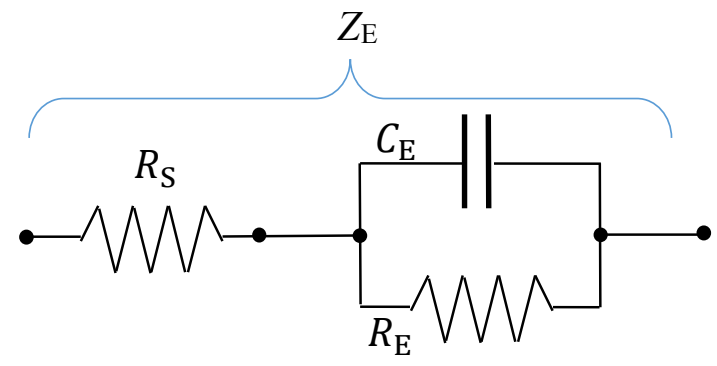

Figura 4-23: Modelo de impedancia de electrodo utilizado para la simulación por el Método de Monte Carlo.

Al igual que para el DRL capacitivo, el peor caso para la estabilidad del sistema fue obtenido mediante una simulación de Monte Carlo, donde se simularon diferentes valores para los parámetros que no pueden ser controlados por diseño, como las capacidades de acoplamiento $C_{\mathrm{B}}, C_{\mathrm{P}}, C_{\mathrm{ISO}}$ y $C_{\mathrm{SUP}}$, combinadas en $C_{\mathrm{TH}}$, así como los parámetros del modelo de impedancia de electrodo $R_{\mathrm{S}}, R_{\mathrm{E}}$ y $C_{\mathrm{E}}$. Dado que los tres electrodos tienen iguales dimensiones, son del mismo material y se colocan en la misma zona del cuerpo, se asume que las impedancias de los electrodos de medida y de realimentación serán similares entre sí: $Z_{\mathrm{EM} 1} \approx$ $Z_{\mathrm{EM} 2} \approx Z_{\mathrm{EG}} \approx Z_{\mathrm{E}}$. La expresión evaluada de $F(s)$. $H(s)$ se muestra en (4-16) y los rangos de variación de los parámetros se resumen en la Tabla 4-3:. Para las capacidades de acoplamiento, los rangos de variación utilizados son los mismos que para el análisis del CDRL, tomados de los resultados de las mediciones mostradas en el Capítulo 2 y publicadas en (Haberman et al. 2011), mientras que los rangos adoptados para los parámetros de la impedancia de electrodo son tomados de la literatura (Levkov1988, Chi et al. 2010a).

$$
F(s) \cdot H(s) \approx \frac{1}{1+s\left(3 C_{\mathrm{IN}}+C_{\mathrm{TH}}\right) \cdot Z_{\mathrm{E}}(\mathrm{s})+s^{2} \cdot C_{\mathrm{TH}} \cdot C_{\mathrm{IN}} \cdot\left(Z_{\mathrm{E}}(s)\right)^{2}}
$$

\begin{tabular}{ccc}
\hline Parámetro & Valor mínimo & Valor máximo \\
\hline $\begin{array}{c}\boldsymbol{C}_{\mathbf{P}}, \boldsymbol{C}_{\mathbf{B}}, \\
\boldsymbol{C}_{\mathbf{S U P}}, \boldsymbol{C}_{\text {ISO }}\end{array}$ & Ver Tabla 4-1 & Ver Tabla 4-1 \\
$\boldsymbol{R}_{\mathbf{S i}}$ & $500 \Omega$ & $1500 \Omega$ \\
$\boldsymbol{R}_{\mathbf{E i}}$ & $300 \mathrm{k} \Omega$ & $1 \mathrm{M} \Omega$ \\
$\boldsymbol{C}_{\mathbf{E i}}$ & $10 \mathrm{nF}$ & $1 \mu \mathrm{F}$ \\
$\boldsymbol{C}_{\mathrm{in}}$ & $10 \mathrm{pF}$ & $10 \mathrm{pF}$ \\
\hline
\end{tabular}

Tabla 4-3: Rangos de variación de los valores utilizados en la simulación de Monte Carlo. Extraidos deHaberman et al. (2011), de Chi et al. (2010a) y de Levkov (1988).

El resultado de la simulación de Monte Carlo, luego de 50000 corridas, fue que el polo más bajo de $F(s) . H(s)$ se ubica por encima de los $750 \mathrm{kHz}$.

La función de transferencia adoptada para $G_{\mathrm{DRL}}$ (s) se muestra en (4-17), la misma presenta una ganancia a la frecuencia de red de aproximadamente $56 \mathrm{~dB}$ y corta el eje de ganancia unitaria en los $32 \mathrm{kHz}$, 
ofreciendo un amplio margen de estabilidad. Si bien la ganancia es exagerada para la interferencia de $50 \mathrm{~Hz}$, hay un beneficio en el incremento que se produce para los armónicos de dicha frecuencia y a la frecuencia de otras fuentes de interferencia como las luces fluorescentes (Winter y Webster 1983), dado que aumenta el ancho de banda de $G_{\mathrm{DRL}}(s)$.

$$
G_{\mathrm{DRL}}(s)=\frac{1}{5 \mu \mathrm{s} \cdot s}
$$
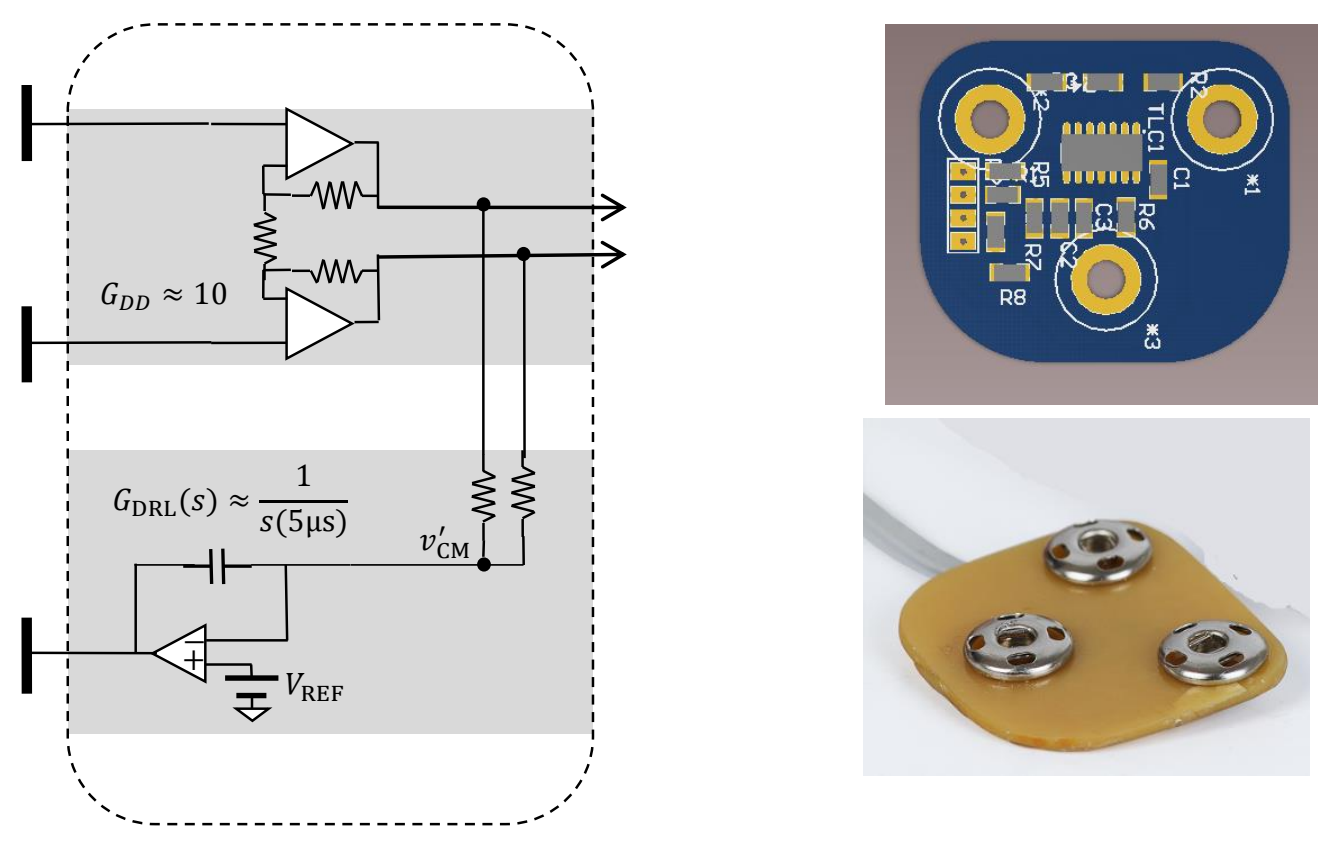

Figura 4-24: (Izquierda) Circuito del Sensor activo de EMG, el cual incluye un circuito DRL que reduce notablemente la tensión $v_{\mathrm{CM}}$. (Derecha arriba) Cara superior del sensor, donde se disponen los componentes de montaje superficial. (Derecha abajo) Cara inferior con los electrodos de acero inoxidable que pueden hacer contacto con la piel o acoplarse a electrodos húmedos comerciales.

\subsection{Conclusiones}

La interferencia de modo común, cuya principal fuente es la red de distribución de energía eléctrica, es una problemática a considerar en la adquisición de biopotenciales y por esto en general son utilizadas topologías de amplificación con muy alto rechazo de modo común. Sin embargo, un muy alto CMRR no es eficaz para eliminar las transformaciones de modo producidas por el efecto divisor de potencial.

Es por esto que se recurre a la conocida técnica de realimentar negativamente la tensión de modo común, reduciendo directamente la tensión interferente, antes de que ingrese al sistema de medida.

El circuito clásico propuesto por (Winter y Webster 1983) para el DRL, presenta una ganancia que en determinadas situaciones resulta escasa. Esta limitación está dada por criterios conservadores en el 
análisis de estabilidad del lazo conformado por el DRL, considerando por ejemplo el uso de filtros de RF y cables blindados. En este sentido, esta tesis aporta un nuevo esquema para la reducción de la tensión de modo común, mediante la utilización de circuitos sintonizados en la frecuencia interferente, que permiten una reducción del modo común mayor a $70 \mathrm{~dB}$, cumpliendo con los criterios de estabilidad del DRL clásico. El circuito fue implementado mediante técnicas digitales como un bloque independiente que puede acoplarse a sistemas de adquisición de biopotenciales preexistentes, mejorando el rechazo de modo común de los mismos y sin comprometer de ningún modo su estabilidad. Dicho circuito demostró, mediante resultados experimentales, ser útil también para acompañar sistemas de adquisición con rechazo de modo común intrínsecamente bajo, como arreglos de canales de EEG Single-Ended o electrodos preamplificados. La utilización de estas topologías permite implementar técnicas para monitorear el estado de las impedancias de electrodo (Levkov 1988).

En los sistemas íntegramente capacitivos se hace necesaria la reducción de la tensión de modo común dado que su CMRR es intrínsecamente bajo. En la literatura pueden encontrarse circuitos donde el CDRL toma ganancias entre 40 y $60 \mathrm{~dB}$, pero rara vez se hace mención a la estabilidad de dichos circuitos realimentados. Además, en algunos casos esas ganancias no son suficientes y se ve también el uso de filtros notch. Mediante simulación por el método de Monte Carlo se obtuvo el rango de posibles valores para $v_{\mathrm{CM}}$, donde se deduce que es necesaria una ganancia para el CDRL de entre 60 y $80 \mathrm{~dB}$. En el presente capítulo se analizó la estabilidad del CDRL considerando factores que aportan a la inestabilidad y que generalmente son obviados en la literatura, como la respuesta en frecuencia de los electrodos capacitivos y las componentes resistivas en serie con la capacidad de los electrodos. El polo de más baja frecuencia a lazo abierto se produce en $800 \mathrm{kHz}$, lo cual permite implementar un CDRL estable con un circuito pasabajos de primer orden con ganancias del orden de los $80 \mathrm{~dB}$ a $50 \mathrm{~Hz}$. Se implementó un prototipo de CDRL que permitió la adquisición completamente aislada tanto de ECG como de EMG.

Se construyó un sensor de EMG que, aprovechando la característica diferencial de la señal de EMG y la cercanía entre electrodos, incluye ambos electrodos de medida junto al amplificador diferencial e incorpora un tercer electrodo a través del cual realimenta la salida de un DRL implementado en el mismo sensor. Esta implementación dará mucha robustez al diseño de un DA controlado por señales de EMG que será presentado en capítulos subsiguientes. 
Capítulo 4 - Reducción de interferencia de modo común 


\section{Capítulo 5}

\section{Procesamiento digital de EMG para Dispositivos de Ayuda}

En el presente capítulo se comentan distintos paradigmas de procesamiento digital de la señal de EMG superficial aplicado a dispositivos de ayuda y su aplicación en dispositivos tipo switch (interruptor o conmutador) controlados por EMG. Se repasan algunas técnicas de procesamiento ON/OFF cuya función es asignar uno de los dos estados posibles (contracción y reposo) a cada segmento analizado de la señal de EMG. En general, los algoritmos para DAs del tipo switch dependen de parámetros obtenidos mediante algún procedimiento de calibración o por ajuste manual, que definen un umbral contra el cual se compara alguna característica de la señal.

En estos casos, variaciones del nivel base 0 de las señales durante la contracción, pueden degradar la performance de los DAs, requiriendo nuevos ajustes de los parámetros de procesamiento. Esto llevó a investigar técnicas que sean robustas ante posibles cambios en las condiciones del nivel base (ruido y señales espurias registradas durante el reposo), y también ante variaciones de los niveles de señal durante las contracciones. Esto último implica que no sea necesario calibrar o ajustar parámetros en ningún momento.

De la investigación surgen dos estrategias adaptivas que no requieren ajuste manual, que fueron aplicadas a un conjunto de registros de EMG de tres músculos distintos. Los resultados son promisorios, y muestran una notable ventaja sobre los métodos de umbral fijo frente a cambios del nivel base. 


\subsection{Introducción}

La señal de EMG superficial, como se explicó en el Capítulo 2 es producto de la activación de distintas unidades motoras de un músculo, que en función de la posición relativa, de la distancia a los electrodos de medida, y de las tasas de disparo, se suman con distintos pesos, componiendo la señal captada por los electrodos.

Estas señales son una muy buena vía para manifestar la voluntad del usuario y controlar una interfaz HombreMáquina. En la medida que se tenga control voluntario sobre un músculo, la persona podrá modular a voluntad la amplitud de la señal de EMG, y esto podrá ser detectado.

\subsubsection{Procesamiento}

El procesamiento de la señal de EMG en DAs implica la realización de operaciones y transformaciones matemáticas sobre las señales captadas por los electrodos, a fin de identificar el mensaje voluntario del usuario, que modula las señales de EMG.

En general, el algoritmo encargado de producir la salida de control no recibe como entrada las señales de EMG superficiales en crudo, sino que estas son procesadas previamente en una serie de bloques (ver Figura 5-1) que mejoran la manera en que los algoritmos identifican la voluntad del usuario.

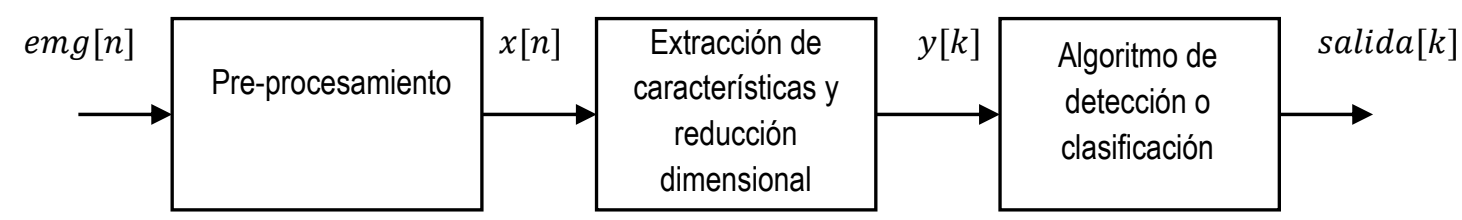

Figura 5-1: Diagrama con los bloques típicos en el procesamiento de la señal de EMG. El objetivo en conjunto es convertir la(s) señal(es) medida(s) en una salida de control.

\subsubsection{Pre-procesamiento}

En primera instancia las señales captadas por los electrodos son sujetas a un pre-procesamiento donde se eliminan las bajas frecuencias, en donde está concentrada la energía de los artefactos de movimiento, mediante filtrado pasaaltos con frecuencias de corte inferior entre 10 y $30 \mathrm{~Hz}$. También se limitan mediante filtrado pasabajos las altas frecuencias, donde el contenido de energía de la señal de EMG disminuye en desmedro de la relación señal ruido (SNR). Dependiendo de la aplicación la frecuencia de corte superior está típicamente entre $200 \mathrm{y}$ $500 \mathrm{~Hz}$. En algunos casos se puede encontrar también filtrado rechazabanda a la frecuencia de red. La implementación de dichos filtros puede realizarse tanto en el dominio analógico como digital, mientras que el resto del procesamiento se restringe al dominio digital.

La salida de este bloque es la señal $x[n]$, que es prácticamente lo que se denomina la señal cruda de EMG.

\subsubsection{Extracción de características}


El punto más crítico es la extracción de características de la señal, que son señales temporales que describen eficientemente el fenómeno en observación, en este caso la voluntad del usuario. Estas se calculan sobre segmentos temporales de la señal de EMG que pueden o no estar solapados. Sobre cada segmento se calcula una o más características (formando un vector de características) que luego serán procesadas por un algoritmo.

En el procesamiento de la señal de EMG encontramos que se utilizan características calculadas directamente en el dominio del temporal como las enumeradas por Jiang et al. (2015) o en Lopez (2010), en el dominio de la frecuencia luego de aplicar alguna transformada o estimar el espectro (Barreto 2000) y en el dominio tiempoescala luego de aplicar la transformada Wavelet (Caamaño et al. 2011).

Los vectores de características suelen contener información redundante, especialmente cuando se trabaja con un gran número de señales y características. En este caso, un paso adicional conveniente es la reducción de dimensionalidad del vector de características, lo cual suele realizarse mediante la proyección en componentes principales (PCA) (Caamaño et al. 2011), y seleccionando aquellas de máxima varianza. También Puede utilizarse el análisis de componentes independientes (ICA) (Stone 2004).

La salida de este bloque es la característica extraída $y[k]$, que puede ser escalar o vectorial.

\subsubsection{Algoritmos de reconocimiento de patrones, clasificadores y detectores de contracción}

Cuando la persona puede realizar distintos movimientos, estos pueden ser detectados para controlar dispositivos con varios grados de libertad como manipuladores robóticos, prótesis mioeléctricas, sillas de ruedas o un cursor en la PC, donde cada movimiento detectado indica un comando diferente.

Si dichos movimientos involucran músculos cercanos, los electrodos de los distintos canales de medida pueden estar ubicados próximos entre si y captar señales cruzadas de los otros músculos (crosstalk) o incluso algunos músculos pueden participar en más de un movimiento. En estos casos es usual el uso de clasificadores para el reconocimiento automático de los distintos patrones de movimiento. Los clasificadores son algoritmos que primero deben ser entrenados con un conjunto de numerosos ejemplos (clasificación supervisada) a partir de los cuales establecerán reglas para luego poder generalizar a nuevos casos y poder asignarles la clase que corresponda. La clasificación de patrones es una disciplina extensa que excede el alcance de la tesis, pudiendo el lector remitirse a la bibliografía específica en el tema (Sierra Araujo 2006).

Cuando los movimientos a detectar involucran músculos diferentes y los electrodos están ubicados de manera que no hay crosstalk, puede obviarse la utilización de clasificadores clásicos, utilizando en su lugar reglas basadas en el conocimiento de los expertos para distinguir entre los distintos movimientos (Moon et al. 2005, Barreto et al. 2000). Un extremo de estas situaciones lo hayamos en las interfaces de tipo switch, donde se desea detectar la activación de un único músculo y la clasificación se reduce a la comparación de alguna característica extraída de la señal contra un umbral.

Otro paradigma para el control de dispositivos está en el uso proporcional de las señales de EMG superficial, dada la relación existente entre la fuerza desarrollada y la amplitud del EMG, en contracciones isométricas (Clancy y Hogan 1997). Un ejemplo de esto es la interfaz publicada por Williams y Kirsch (2008) que puede controlar el desplazamiento del puntero del mouse con dos canales de EMG. La velocidad en cada eje es proporcional a la energía de la señal de EMG en un canal determinado.

\subsection{Dispositivos de Ayuda tipo switch}

La aplicación más intuitiva de la señal de EMG para el control de DAs es a través de su utilización como señal binaria (ON/OFF). En la Figura 5-4(a) se ilustra parte de un registro de EMG y la salida ON/OFF surgida del 
procesamiento de dicha señal. A pesar de proveer una señal de control de sólo un bit, sus aplicaciones pueden extenderse mediante interfaces hombre-máquina $(\mathrm{HMI})$ secuenciales como menús y punteros auto-deslizantes 0 teclados de barrido (por ejemplo Gazetalk de Cogain (Online), Plaphoons y otros programas del Proyecto Fressa (Online)). Cuando el usuario además controla voluntariamente el tiempo que puede mantener la contracción, es posible incrementar el número de símbolos que pueden representarse codificándolos por duración (Park et al. 1999). De este modo, con solo controlar la contracción voluntaria de un músculo, es posible la obtención de una señal binaria que, complementada mediante alguna interfaz de usuario, permite realizar tareas complejas como escribir, comunicarse, desplazarse, activar luces, levantar el respaldo de una cama o cambiar de canal, entre innumerables acciones más.

Este tipo de DA puede brindarle a una persona con discapacidades motrices severas (como pacientes con ELA, lesiones de médula espinal, o víctimas de ACV) una mejora significativa en su calidad de vida. Si la persona conserva parte del control voluntario de la contracción de algún músculo, aunque no logre realizar una contracción intensa, estará modulando a voluntad la señal de EMG producida en dicho músculo. De hecho, junto con las $\mathrm{BCls}$ son una de las pocas maneras de comunicarse en estados avanzados de ELA (Hansen et al. 2000). Una alternativa interesante son las interfaces híbridas (Leeb et al 2011, Müller-Putz 2011) que combinan ambos tipos de señales. Así, puede utilizarse como primera opción EMG, y cuando el usuario se fatiga, y ya no es posible operar en este modo, pasar a utilizar EEG; resignando velocidad pero manteniendo la comunicación. Del mismo modo, el EMG es una de las señales recomendadas para control de una PC en personas con lesiones de la médula espinal, dado que aún en las lesiones más graves de la primera vértebra cervical se conserva el control de los músculos faciales (Bates 2002).

Tal es la potencialidad de estas señales que se encuentran opciones comerciales de switches controlados por señales de EMG, como el "EMGSwitch" de Tinkertron (Andreasen 2005) (ver Figura 5-2(a)) que posee como salidas interruptores físicos (relés), mientras que otros equipos requieren de una PC en donde el "switch" activa opciones en un menú secuencial como el "NeuroSwitch" de Controlbionics (Online) (ver Figura 5-2(b)) o el "Headband-Operated EMG Switch" (CanAssist Online).

(a)

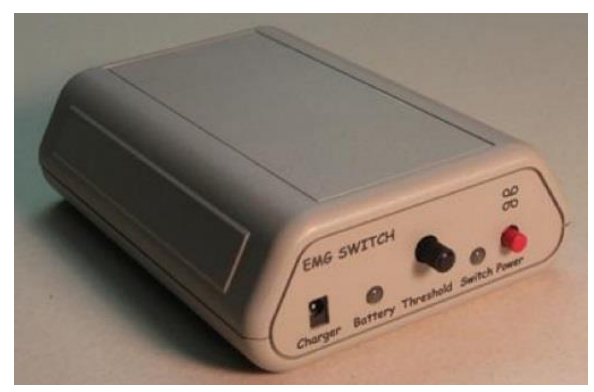

(b)

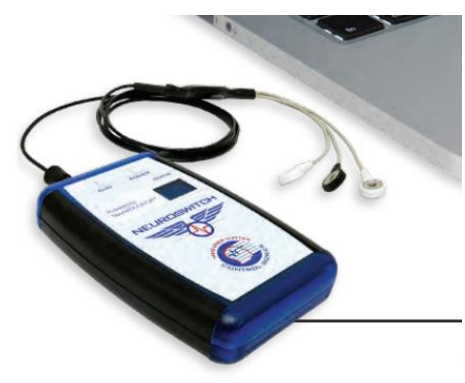

Figura 5-2: Switchs controlados por EMG de circulación comercial: (a) EMGSwitch de Tinkertron. (b) NeuroSwitch de Controlbionics. 


\subsubsection{Técnicas existentes}

La obtención de una señal ON/OFF es de interés no solo en switches, sino también en dispositivos más complejos como prótesis mioeléctricas de velocidad constante (con control ON/OFF). También es de interés en estudios neurológicos, investigación neuromuscular y psicomotora, medicina del deporte y rehabilitación (Staude et al. 2001). Curiosamente, el procesamiento ON/OFF de la señal de EMG en dispositivos de ayuda, en la mayoría de las publicaciones, no implementa las soluciones surgidas en otras áreas de investigación, como técnicas para la detección automática del principio de la contracción, las cuales tienen gran desarrollo. Se trata de técnicas más metódicas y con basamentos teóricos más sólidos que buscan encontrar en la señal de EMG el momento exacto donde el músculo pasa del estado de reposo al de contracción (algunas también buscan la transición opuesta). En general, en las Interfaces Hombre-Máquina se utilizan soluciones heurísticas menos formales.

\subsubsection{Detección del principio de la contracción}

Un campo muy específico del conocimiento sobre el procesamiento de la señal de EMG es la detección del comienzo de la contracción (Jiang et al. 2015, Caamaño et al. 2011, Staude 2001, Hodges y Bui 1996).

Como se explica en (Staude et al. 2001) en estas técnicas es usual encontrar una comparación de la amplitud o características similares en el dominio temporal, como el valor RMS (Root Mean Square) o el MAV (Mean Absolute Value) con un umbral determinado a partir de las condiciones de reposo (ver Figura 5-3). Su simplicidad facilita la implementación en tiempo real y tienen buen desempeño con SNR mayores a $10 \mathrm{~dB}$.

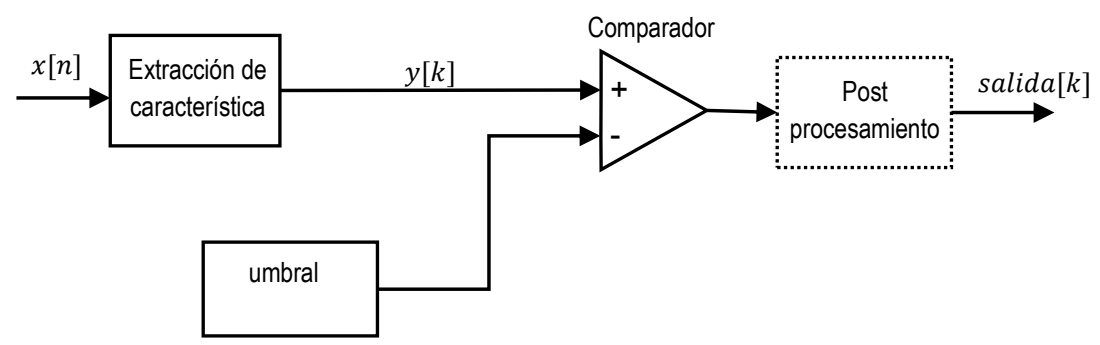

Figura 5-3: Esquema del procesamiento típico para la detección de contracciones.

Señales típicas pueden verse en la Figura 5-4(b), donde se ilustra la comparación entre un umbral y la característica $y[k]$ (el MAV) calculado sobre los segmentos de la señal cruda de EMG $x[k]$ graficada en Figura 5-4(a). 


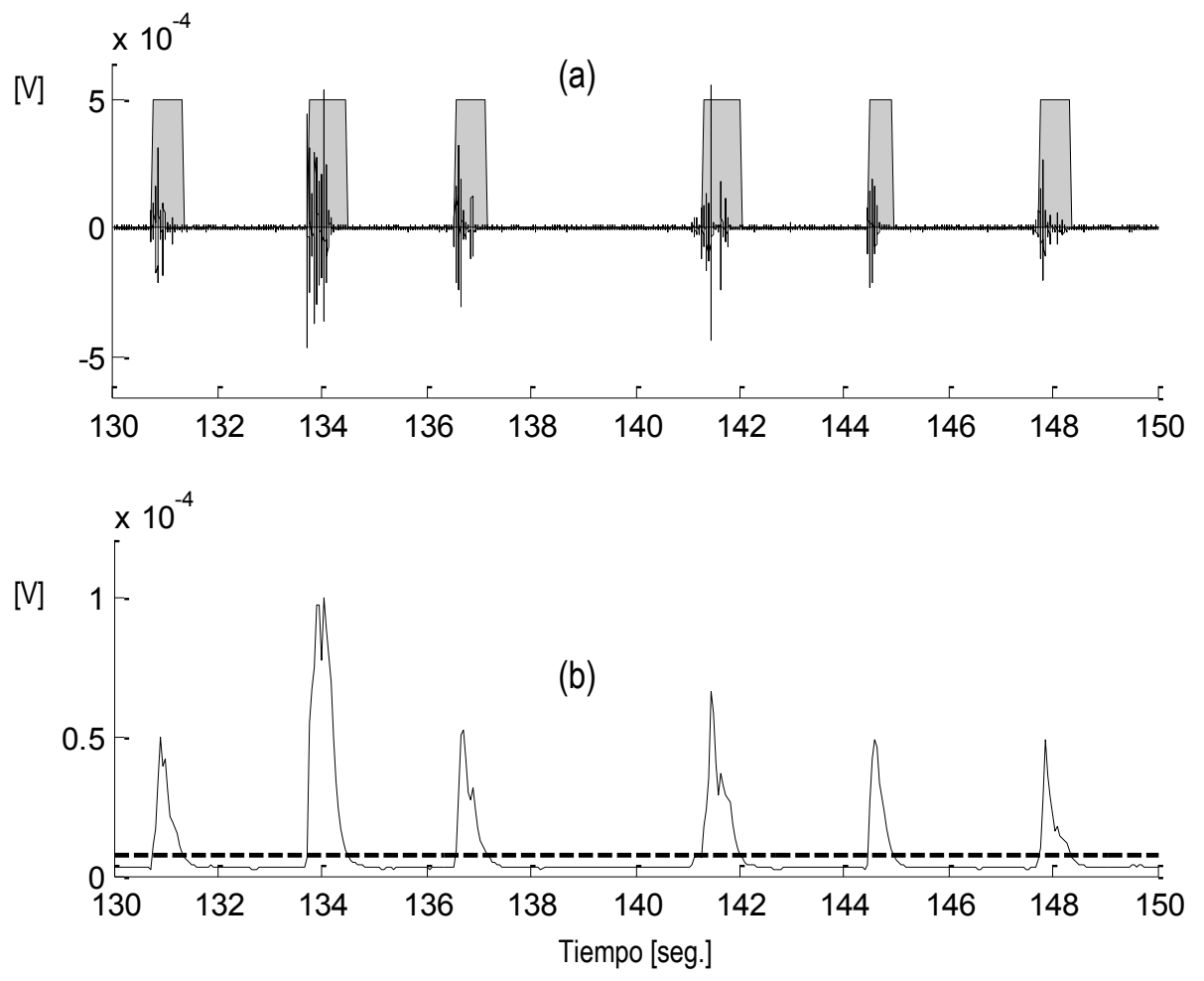

Figura 5-4: (a) Señal de EMG cruda $x[k]$ y salida binaria salida $[k]$, donde el nivel alto corresponde al estado de contracción y el nivel bajo al estado de reposo. (b) detalle interno del procesamiento donde se compara la característica $y[k]$ con un umbral predefinido.

Para establecer dicho umbral suele usarse (5-1), donde media $a_{y}$ es la media muestral de la característica $y[k]$, mientras que desviación $n_{y}$ es la desviación estándar de $y[k]$. Ambos valores son calculados durante un período en reposo (Jiang et al. 2015, Staude et al. 2001, Hodges y Bui 1996). Por su parte, $K$ es una constante arbitraria, que recibe diferentes valores según cada autor.

$$
\text { umbral }=\text { media }_{y}+K * \text { desviación } n_{y}
$$

Otras técnicas propuestas en la literatura involucran marcos teóricos más sólidos para encontrar el comienzo de la contracción como Teoría de la Información (Lopez 2010), descomposición en componentes principales y transformada Wavelet (Merlo et al 2003, Caamaño et al. 2011).

Las técnicas basadas en el procesamiento estadístico de señales (Niemenlehto 2006, Staude et al. 2001, Staude y Wolf 1999) rechazan la hipótesis nula $H_{0}$ (reposo) en favor de la hipótesis alternativa $H_{1}$ (contracción) comparando un test estadístico (generalmente la relación logarítmica de verosimilitud) con un umbral. Dicho umbral es determinado en función del conocimiento "a priori" de las distribuciones de probabilidades bajo ambas hipótesis. 


\subsubsection{Otras técnicas para el procesamiento ON/OFF de contracciones}

Existen estrategias que detectan múltiples patrones de movimiento a partir de distintos canales (Barreto et al. 2000) utilizando características en el dominio de la frecuencia (el periodograma, como aproximación de la PSD), extrayendo la energía en distintas bandas y comparando con umbrales arbitrarios para determinar la presencia 0 ausencia de contracción.

Park et al. (1999) Implementa un comunicador de código morse con un canal EMG. El procesamiento es ON/OFF comparando la varianza de la señal con un umbral arbitrario. Los símbolos raya-punto se distinguen según su duración. En (Rani y Sarkar, 2005) se utilizan tres canales distintos de señal EMG (raya, punto y otro de propósito general) pero cada uno es en sí mismo un switch, donde el valor RMS es comparado con un umbral surgido de un algoritmo de optimización también utilizado en (Nilas y Sarkar, 2004).

Aso et al. (2006) controla una silla de ruedas con comandos producidos por canales independientes de EMG convertidos en señales binarias a partir de la comparación de la varianza con umbrales arbitrarios. Por ejemplo, Fischer et al. (2005) presenta una aplicación para el control del cursor de PC controlada por un único canal de EMG, donde debe ajustarse manualmente el umbral contra el cual se compara su amplitud para generar señales ON-OFF. En general, dada la variabilidad que presentan las señales de EMG frente a numerosos factores, se observa que se escoge un umbral empíricamente (Tsui et al. 2007, Moon et al. 2005) lo cual ocurre también en los switches comerciales de Tinkertron (Andreasen, 2005), que cuentan con una perilla de ajuste de umbral.

Un caso particular a resaltar es el de la solución propuesta por William y Kirsch (2008) que se diferencia de las anteriores por proponer un umbral móvil que depende del nivel base de los últimos 10 segundos y de la máxima contracción voluntaria (MVC) realizada durante una sesión de entrenamiento.

\subsection{Técnicas propuestas para DAs tipo switch}

Cómo puede observarse en los antecedentes descritos, el problema clave en estos DAs es la elección de un umbral adecuado que detecte las contracciones sin producir demasiadas "falsas contracciones". Este punto se tratará en detalle en una sección posterior.

La metodología general es estimar una característica representativa de la contracción, por ejemplo el valor RMS de la señal, y compararlo contra un umbral que se ajusta mediante un algoritmo o manualmente. El umbral queda determinado en función de los niveles de señal, ruido y EMI presentes en el momento del ajuste. Esto suele funcionar correctamente en el corto plazo y bajo condiciones controladas, pero plantea la necesidad de recalibrar los parámetros de procesamiento o reajustar un umbral cada vez que cambien las condiciones de uso. En la Figura 5-7 puede verse un ejemplo de variación en los niveles de ruido en el estado de reposo, donde los primeros segundos de registro presentan un nivel de señal de amplitud considerablemente menor al del resto del registro, que lejos de ser estacionario, muestra una tendencia a aumentar con el tiempo. En el caso particular del ejemplo, el registro se tomó con electrodos húmedos descartables y es probable que al comenzar las sucesivas contracciones el contacto entre los electrodos y la piel se fuese degradando, aumentando los niveles de ruido.

A pesar de las variaciones que puede sufrir la señal durante el reposo, la mayor variabilidad se da en las características de la señal de EMG durante la contracción. Debe recordarse que la misma es una suma de MUAPS de distintas unidades motoras a distintas profundidades, reclutadas progresivamente y con diferentes tasas de disparo. Por lo tanto dichas señales se verán alteradas por muchos factores, como el nivel de contracción, la ubicación y tamaño de los electrodos, movimiento de los electrodos relativo al músculo, posibles estados 
patológicos y fatiga. Así mismo habrá una notable diferencia entre las señales generadas por distintos músculos y entre distintos sujetos.

Un ejemplo de esto puede verse en la Figura 5-5, donde se muestran dos segmentos de señales de EMG del músculo facial cigomático mayor. Uno de los segmentos corresponde a una persona sana y el otro a una persona con ELA. En el segundo caso se pueden observar señales mucho más débiles durante las contracciones voluntarias donde se pueden identificar los MUAPS individuales.

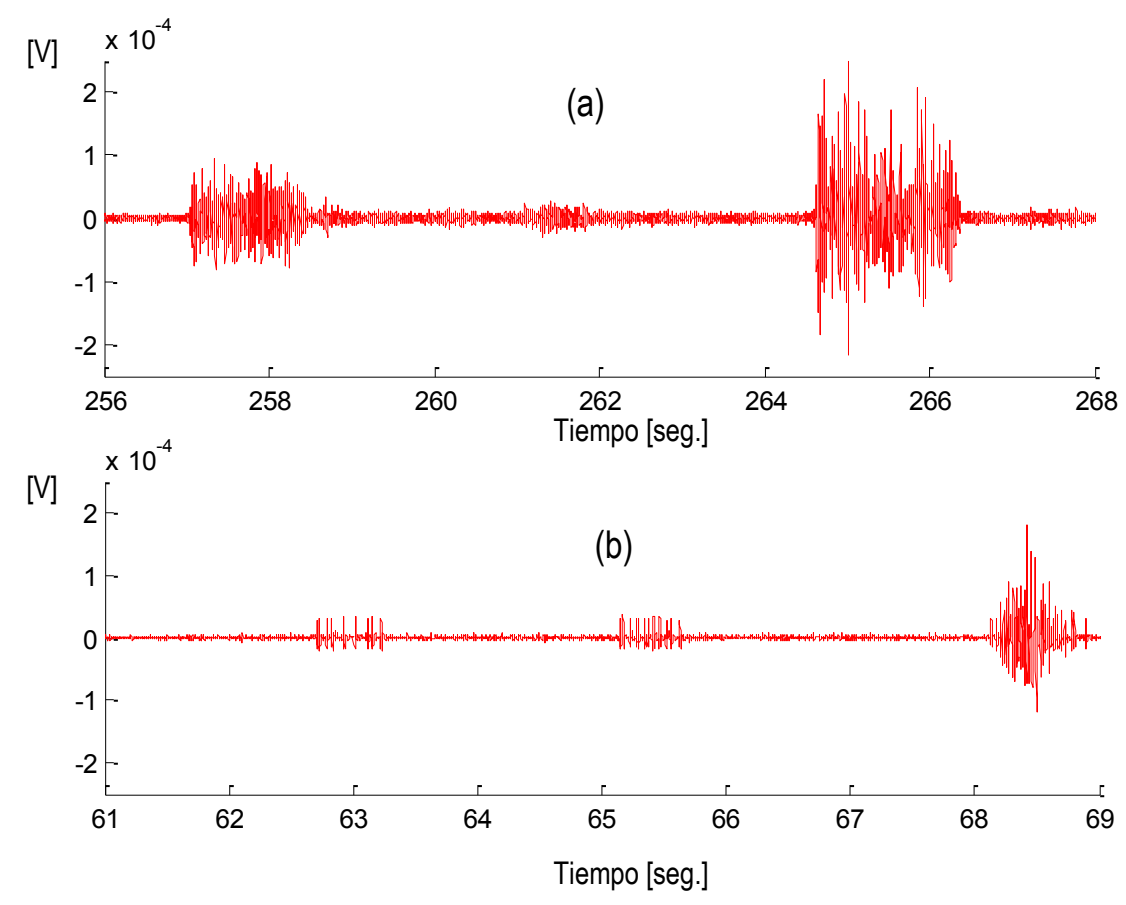

Figura 5-5: Segmentos de registros reales de EMG tomados sobre el músculo cigomático mayor (a) de una persona sana (registro $\mathrm{N}^{\circ}$ 12) (b) de una persona con ELA (registro mencionado al final del capítulo). Para poder compararlas, ambas escalas horizontales y verticales son iguales.

Para lograr un DA que sea aceptado por el usuario, es necesario que sea confiable (que brinde la funcionalidad esperada cuando se lo requiera), pero esto solo no es suficiente. El mismo debe ser robusto ante diversas condiciones del contexto, fácil de operar y también fácil de instalar. Un equipo que para funcionar bien necesite que se lo re-calibre frecuentemente o que requiera largas sesiones de entrenamiento por parte del usuario, difícilmente logre su aceptación. Además el costo de las sesiones con un profesional puede resultar muy superior al costo del equipo.

Por todo esto se pensaron técnicas de procesamiento que no requieran sesiones de entrenamiento ni ajuste manual de parámetros. Es decir, que permitan implementar DAs que al ser colocados sobre el usuario, estén operativos inmediatamente sin requerir ninguna acción adicional por parte de él ni de un tercero. También, debe tenerse en cuenta la necesidad de operar en tiempo real, en sistemas embebidos con recursos limitados de procesamiento y energía.

Las técnicas propuestas utilizan características relacionadas con la amplitud de la señal porque son buenos descriptores del nivel de contracción y simples de calcular en tiempo real (Niemenlehto 2006, Staude et al. 2001) aún en microcontroladores de gama baja. Pero, para determinar la presencia de contracción, en lugar de utilizar 
un umbral estático, la comparación se realiza con un umbral que se adapta continuamente a las distintas condiciones que presente la señal, resolviendo así los problemas de deriva de la señal durante el reposo. Un antecedente de estas soluciones puede encontrarse en una $\mathrm{BCl}$ basada en ritmo alfa visual (Spinelli y Mayosky 2000).

Se proponen en esta tesis dos técnicas diferentes que, ante el cálculo de una nueva muestra de la característica $y[k]$, actualizan el valor de los umbrales de comparación según las condiciones de la señal. Ambas estrategias se enmarcan en el esquema general de la Figura 5-6, donde la salida es binaria y está precedida por un bloque de post-procesamiento cuya finalidad es filtrar transiciones espurias.

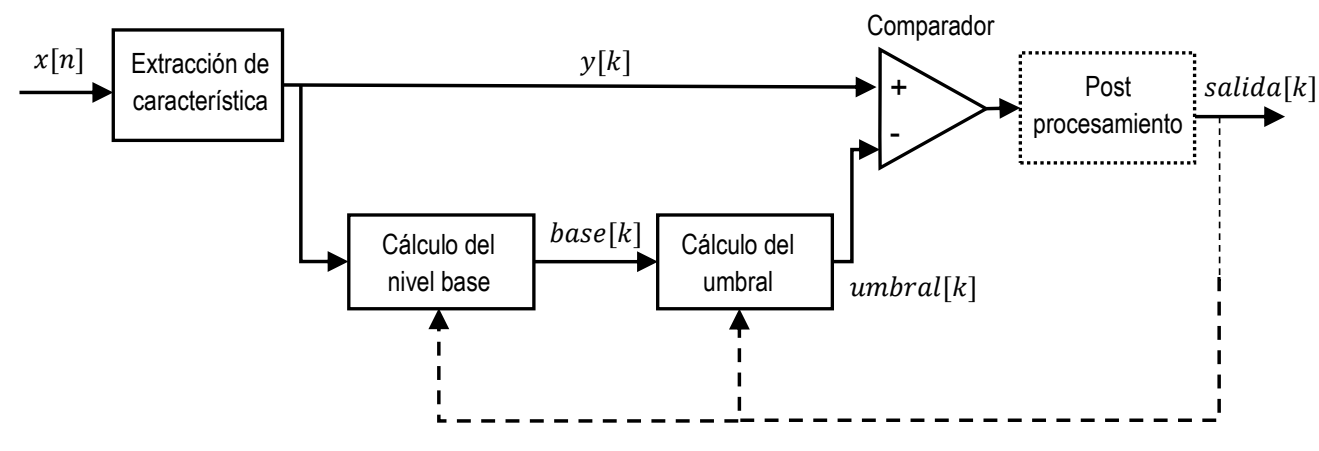

Figura 5-6: Diagrama en bloques general para las técnicas propuestas.

\subsubsection{Extracción de la característica}

Los ensayos se realizaron tomando 1000 muestras por segundo de la señal de EMG cruda, filtrando las frecuencias por debajo de $30 \mathrm{~Hz}$ para rechazar los artefactos de movimiento y por encima $250 \mathrm{~Hz}$ para limitar el ruido, obteniéndose la señal $x[n]$. Para extraer sus características, esta señal se segmenta en ventanas de $50 \mathrm{~ms}$ sin solapamiento ( $\mathrm{N}=50$ muestras), y en cada ventana se calcula el MAV, dado por:

$$
y^{\prime}[k]=\frac{1}{N} \sum_{n=k N+1}^{k N+N}|x[n]|
$$

Siendo $y^{\prime}[k]$ una señal que se actualiza a una tasa de 20 muestras por segundo 5 .

Luego se obtiene la característica $y[k]$ como una versión suavizada de $y^{\prime}[k]$, mediante un filtro IIR que promedia la señal con una longitud equivalente a $150 \mathrm{~ms}$ ( $M=3$ muestras)

$$
y[k]=\left(1-\frac{1}{M}\right) y[k-1]+\frac{1}{M} y^{\prime}[k]
$$

\footnotetext{
${ }^{5}$ Notar que la tasa o frecuencia de muestro de la característica y[k] es mucho menor que la correspondiente a la señal de EMG, y está de acuerdo a la dinámica asociada a la capacidad del usuario generar activaciones, típicamente del orden de 60 por minuto.
} 
Además del MAV también fueron ensayados otros descriptores de la señal de EMG (valor RMS, envolvente, longitud, cantidad de cruces por cero y mediana) pero de todos estos, los que se destacaron por ser más robustos ante las simulaciones de ruido e interferencia fueron el MAV, el valor RMS y la envolvente. Los resultados fueron similares. Se escogió el MAV, debido a su facilidad de cómputo.

\subsubsection{Estimación del nivel base}

Para definir el umbral, es necesario establecer cuál es el nivel de ruido en reposo (o nivel base). Este se estima durante los períodos clasificados como reposo, pasando la característica $y[k]$ por un filtro pasabajos con diferentes constantes de tiempo, según la señal de entrada $y[k]$ sea mayor o menor que la última estimación del nivel base:

$$
\begin{gathered}
\operatorname{base}[k]=\left\{\begin{array}{cl}
\left(1-K_{\mathrm{BD}}\right) . \text { base }[k-1]+K_{\mathrm{BD}} \cdot y[k], & y[k]<\text { base }[k-1] \\
\left(1-K_{\mathrm{BA}}\right) . \text { base }[k-1]+K_{\mathrm{BA}} \cdot y[k], & y[k] \geq \text { base }[k-1]
\end{array}\right. \\
K_{\mathrm{BD}} \gg K_{\mathrm{BA}}
\end{gathered}
$$

Los valores de las constantes utilizadas en (5-4) se establecieron en $K_{\mathrm{BA}}=\frac{50 \mathrm{~ms}}{10 \mathrm{~s}}$ y $K_{\mathrm{BD}}=\frac{50 \mathrm{~ms}}{150 \mathrm{~ms}}$.

De esta manera, al ser $K_{\mathrm{BD}} \gg K_{\mathrm{BA}}$, base $[k]$ sigue a $y[k]$ con una dinámica mucho más veloz en sentido descendente que ascendente, y de este modo base $[k]$ sigue el valor mínimo de $y[k]$.

Durante la contracción, por el contrario, el valor de base $[k]$ permanece constante.

$$
\operatorname{base}[k]=\operatorname{base}[k-1]
$$

Mientras que al retornar del estado de contracción al estado de reposo la estimación del nivel base se reestablece, igualándolo al valor de la característica.

$$
\operatorname{base}[k]=y[k]
$$

En la Figura 5-7 puede verse la señal $y[k]$ con cambios en su nivel base, junto a la estimación de su nivel base. 


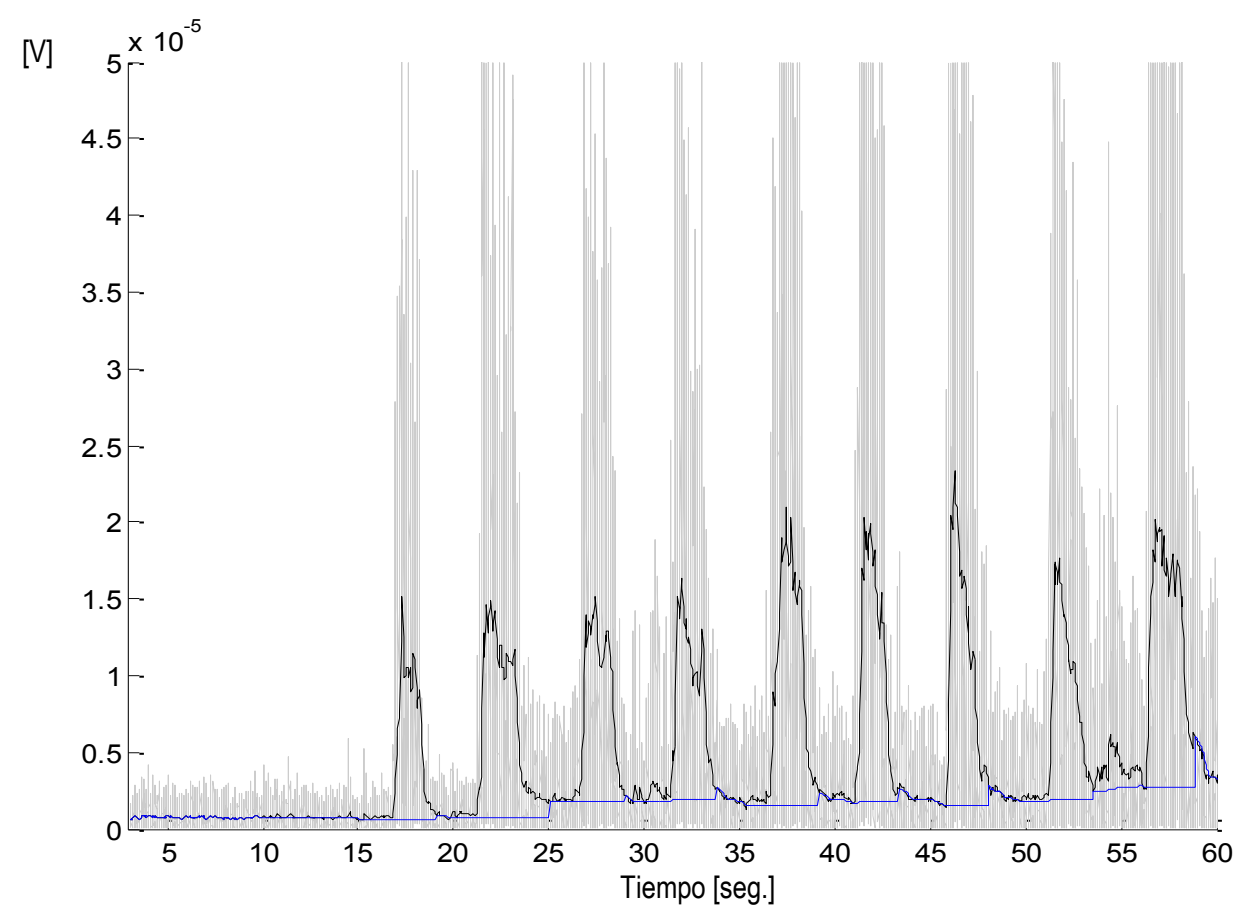

Figura 5-7: Señal de EMG del músculo frontal (corresponde al registro $N^{\circ} 7$, que se analiza al final del capítulo) donde se observa la variación del nivel base respecto de los primeros 15 segundos del registro. En gris: la señal de EMG rectificada: $|x[n]|$. En negro: la característica $y[k]$ (el MAV). En azul: estimación del nivel base: base $[k]$.

\subsubsection{Cálculo del umbral}

Se proponen aquí dos técnicas para calcular el valor umbral a partir del nivel base.

\subsubsection{Técnica I}

En esta técnica derivada de (Spinelli y Mayosky 2000) el umbral se computa como un múltiplo del nivel base:

$$
\text { umbral }[k]=K_{\mathrm{UI}} \cdot \text { base }[k-1]
$$

La constante $K_{\mathrm{UI}}$ se ajustó empíricamente de manera de optimizar el valor de la métrica $H F D$ (la cual se explica más adelante) aplicando el procesamiento a un conjunto de registros de señales EMG reales. Los valores hallados para $K_{\mathrm{UI}}$ son dependientes de la característica utilizada, de las constantes $K_{\mathrm{BA}}$ y $K_{\mathrm{BD}}$ utilizadas para calcular el nivel base en (5-4), y de la frecuencia de muestro de $y[k]$.

Para el caso particular presentado la constante se ajustó en $K_{\mathrm{UI}}=3$.

\subsubsection{Tecnica II}

En esta técnica el umbral se computa como la suma del nivel base con un múltiplo de la desviación estándar de $y[k]$ durante el reposo.

$$
\text { umbral }[k]=\operatorname{base}[k-1]+K_{\mathrm{UII}} \cdot \operatorname{desv}[k-1]
$$


Esto surge del trabajo de (Di Fabio 1987) y en otros reunidos por (Hodges y Bui 1996), destinados en detectar el comienzo de contracciones, donde el umbral se determina según (5-1) como el promedio de la característica $y[k]$ durante un período en reposo, más una cantidad $K$ de desviaciones estándar de dicha señal.

En la presente técnica se mantiene esta forma de cómputo del umbral, pero en lugar de valores constantes para la media y la desviación estándar de $y[k]$, se utilizan respectivamente la estimación actualizada del nivel base y una estimación continua de la desviación $\operatorname{desv}[k]$. Dicho cálculo se actualiza en estado de reposo, solo si $y[k]$ es mayor que base [k-1], mediante un filtro pasabajos, con una constante de tiempo equivalente de 3 minutos $\left(K_{\mathrm{D}}=\frac{50 m s}{180 s}\right)$.

$$
\operatorname{desv}[k]=\left\{\begin{aligned}
\operatorname{desv}[k-1], & y[k]<\operatorname{base}[k-1] \\
\sqrt{\left(1-K_{\mathrm{D}}\right) \operatorname{des} v[k-1]^{2}+K_{\mathrm{D}}(y[k]-\operatorname{base}[k-1])^{2},} & y[k] \geq \operatorname{base}[k-1]
\end{aligned}\right.
$$

Una versión más simple del estimador para implementar en microcontroladores con pocos recursos es el cálculo de la deviación de manera lineal se ve en (5-10), de hecho, mostró leves ventajas respecto de la expresión (5-9).

$$
\operatorname{desv}[k]=\left\{\begin{aligned}
\operatorname{desv}[k-1], & y[k]<\operatorname{base}[k-1] \\
\left(1-K_{\mathrm{D}}\right) \operatorname{desv}[k-1]+K_{\mathrm{D}} \mid y[k]-\operatorname{base}[k-1], & y[k] \geq \operatorname{base}[k-1]
\end{aligned}\right.
$$

La constante $K_{\mathrm{UII}}$ se ajustó empíricamente, de la misma manera que $K_{\mathrm{UI}}$, en $K_{\mathrm{UII}}=12$.

En las Figura 5-8(b) y Figura 5-9(b) pueden verse los umbrales calculados por ambos métodos en comparación con un umbral fijo calculado con (5-1) sobre los primeros 10 segundos con $K=K_{U I I}=12$, para diferentes registros reales de EMG. Se observa que para un nivel base estacionario las técnicas propuestas muestran el mismo buen desempeño que utilizando un umbral fijo (Figura 5-9), pero la fortaleza de las mismas se aprecia en la capacidad para adaptarse a los cambios en el nivel base de la señal (Figura 5-8) donde los métodos de umbral fijo fallan. 

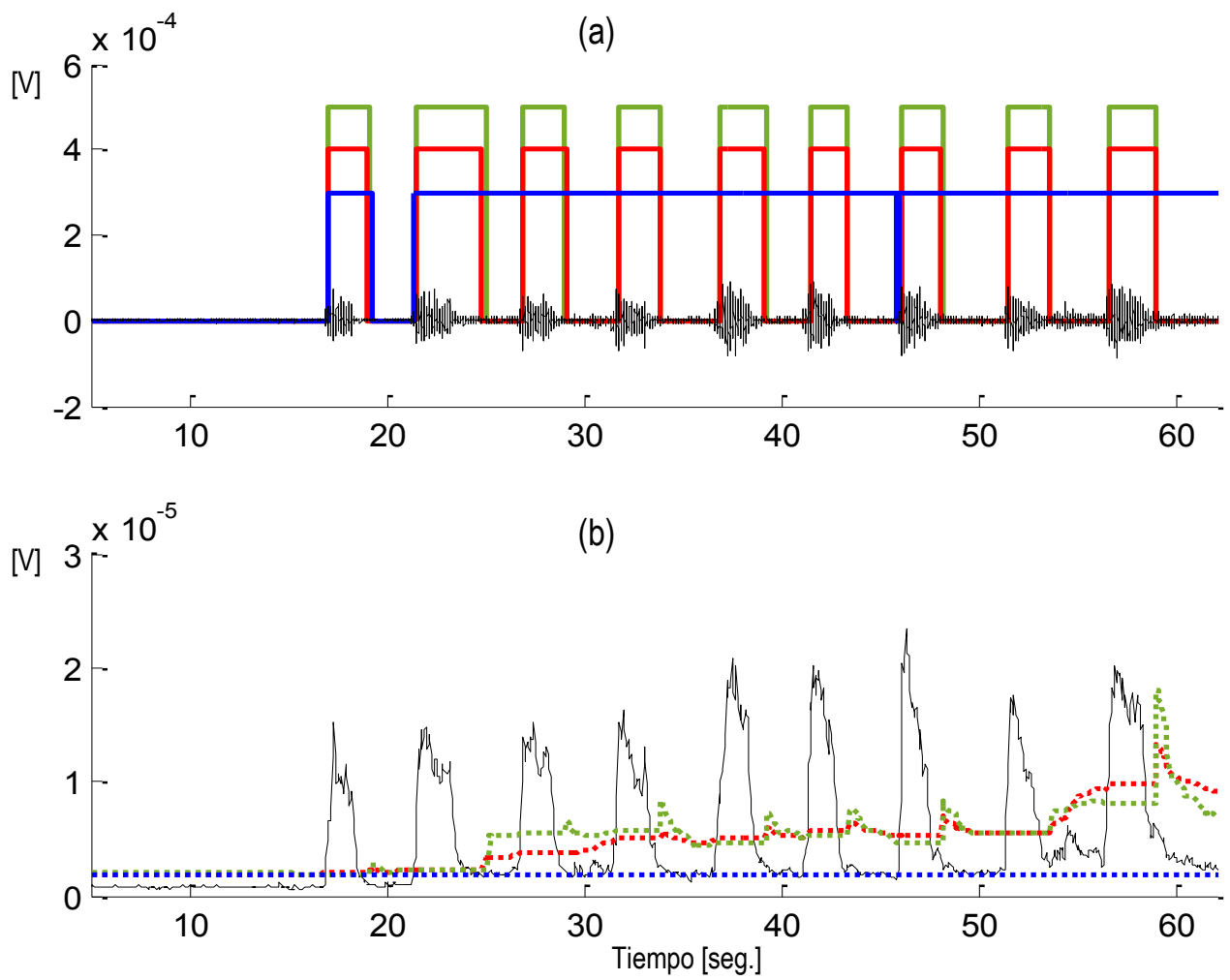

Figura 5-8: (a) Señal de EMG cruda $x[k]\left(\right.$ Reg. $\left.N^{\circ} 7\right)$ y salidas binarias salida $[k]$ (azul: comparando con umbral fijo, verde: Técnica I, rojo: Técnica II) (b) característica $y[k]$ y umbral $[k]$ (azul: umbral fijo, verde: Técnica I, rojo: Técnica II) 


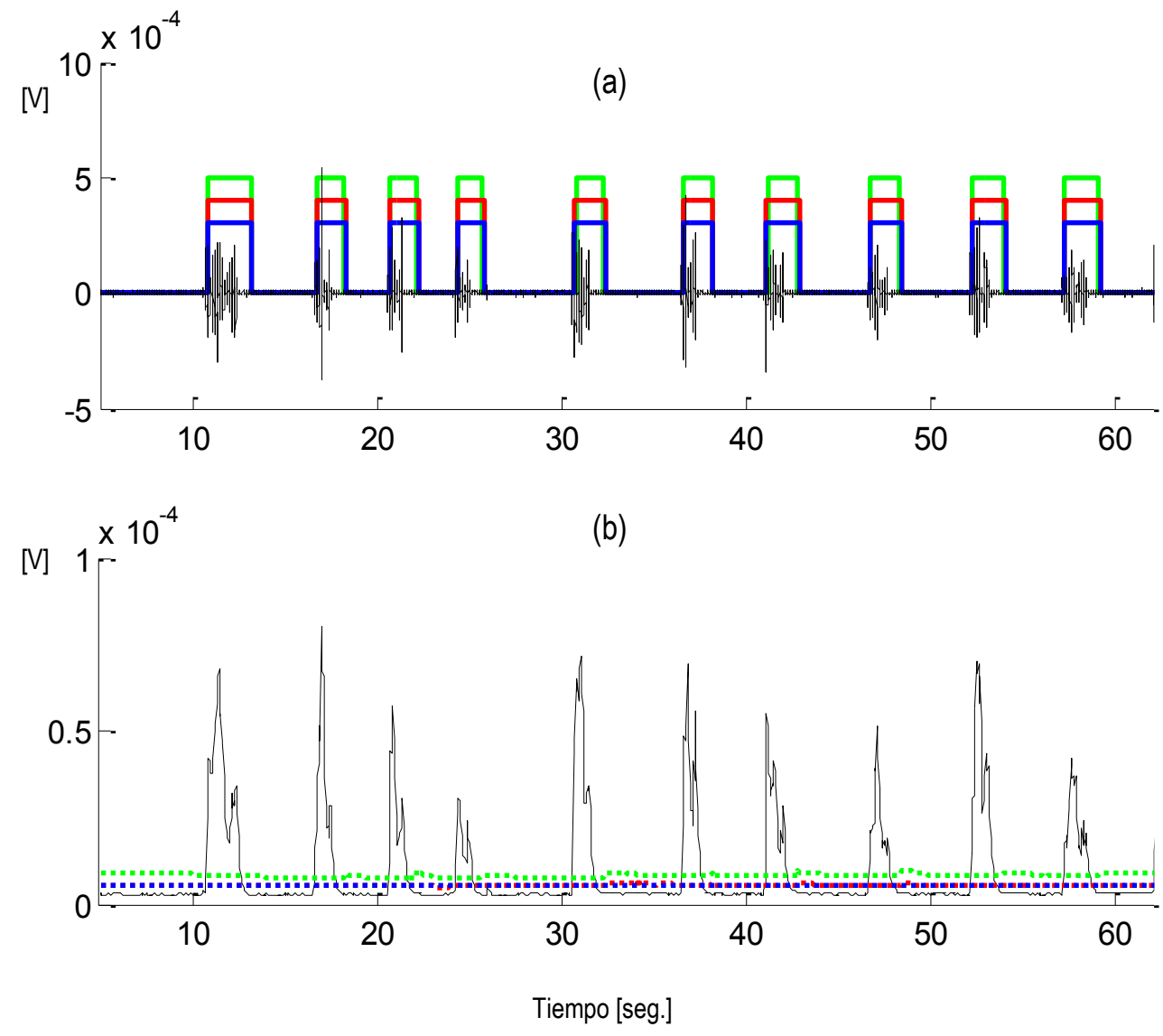

Figura 5-9: (a) Señal de EMG cruda $x[k]\left(\right.$ Reg. $\left.N^{\circ} 4\right)$ y salidas binarias salida $[k]$ (azul: comparando con umbral fijo, verde: Técnica I, rojo: Técnica II) (b) característica $y[k]$ y umbral $[k]$ (azul: umbral fijo, verde: Técnica I, rojo: Técnica II).

\subsubsection{Post-procesamiento}

Si bien $y[k]$ se obtiene mediante un suavizado, la comparación con un único umbral implica que puedan existir "rebotes" o transiciones espurias de un estado al otro hasta lograr un estado estable. Esto puede evitarse utilizando dos umbrales, aplicando así una franja de histéresis en la comparación (Spinelli y Mayosky 2000).

Una solución más completa para evitar transiciones espurias y otros comportamientos improbables para la dinámica de la señal de EMG, es utilizar un bloque de post-procesamiento a la salida, que valide las distintas transiciones según un conjunto de reglas temporales similar a las utilizadas en el EMGSwitch de Tinkertron (Andreasen 2005).

De esta manera, la primera regla utilizada establece un tiempo de mantenimiento $T_{\mathrm{RC}}$ durante el cual $y[k]$ debe ser mayor al umbral para validar la transición al estado de contracción. Este valor fue establecido en $T_{\mathrm{RC}}=$ $150 \mathrm{~ms}$. Dado que es improbable que una contracción que dure menos que un segundo, se podría incrementar aún más dicho tiempo, pero debe hacerse con precaución dado que sería en desmedro del tiempo de respuesta.

Del mismo modo, para pasar del estado de contracción al de reposo, $y[k]$ debrá mantenerse por debajo del umbral durante un tiempo mínimo $T_{\mathrm{CR}}$. Este tiempo se ajusta en función del tiempo de reposo mínimo esperado entre contracciones consecutivas. En nuestro caso se estableció $T_{\mathrm{CR}}=500 \mathrm{~ms}$. 
Así mismo, se utilizó una tercera regla que previene que, ante un aumento sustancial del ruido, el sistema quede enclavado en el estado de contracción. Esto se logra estableciendo un tiempo máximo permitido para las contracciones $T_{\mathrm{CMAX}}$ luego del cual se retorna al estado de reposo. Este tiempo depende de la aplicación, pero difícilmente se requieran transiciones de más de 5 segundos.

\subsubsection{Evaluación de las técnicas propuestas}

\subsubsection{Registros realizados}

A fin de testear y validar los algoritmos, se tomaron 12 registros de EMG sobre diferentes músculos de un sujeto de sexo masculino de 27 años. Los músculos sobre los cuales se tomaron los registros fueron el bíceps brachii izquierdo (BBI), el frontal derecho (FD) y el cigomático mayor derecho (ZMD).

Los electrodos fueron colocados según las recomendaciones de la SENIAM sobre el BBI y de las referencias (Fridlund y Cacioppo 1986, Tassinary et al. 1989) para el FD y el ZMD. En todas las medidas se utilizaron electrodos húmedos descartables de $\mathrm{Ag}-\mathrm{AgCl}$ con un área de $1,5 \mathrm{~cm}^{2}$ y una distancia inter-electrodos de $2 \mathrm{~cm}$. Se utilizaron electrodos activos, para reducir la incidencia de EMI.

El sujeto realizó numerosas contracciones voluntarias decidiendo arbitrariamente la intensidad y la duración de cada una. Se le pidió que al comenzar cada contracción pulse una tecla del teclado y la mantenga presionada hasta finalizar la misma. De esta manera por cada registro de EMG se dispone de un registro de referencia que permite comparar la voluntad del sujeto con la salida de los distintos algoritmos utilizados.

\subsubsection{La métrica utilizada: HFD}

A fin de poder comparar los distintos algoritmos, se ha utilizado como métrica la diferencia entre la sensibilidad de detección y la proporción de falsos positivos (HFD: Hit-False-Difference) (Schlögl et al. 2007) la cual permite resumir en único índice el desempeño de los algoritmos. Esta se calcula mediante la (3-1).

$$
\begin{gathered}
H F D=H-F \\
H=\frac{N_{\mathrm{BD}}}{N_{\mathrm{T}}} \cdot 100 \% \quad F=\frac{N_{\mathrm{TD}}-N_{\mathrm{BD}}}{N_{\mathrm{TD}}} \cdot 100 \%=\frac{N_{\mathrm{E}}}{N_{\mathrm{TD}}} \cdot 100 \%
\end{gathered}
$$

Donde $H$ (la sensibilidad) representa el porcentaje de eventos (contracciones) bien detectadas $N_{\mathrm{BD}}$ sobre el total de contracciones voluntarias realizadas $N_{T}$, y $F$ es el porcentaje de detecciones erróneas $N_{\mathrm{E}}$ sobre el total de contracciones detectadas $N_{\mathrm{TD}}$.

De esta manera los valores de HFD variaran desde $-100 \%$ en caso de que no se detecte ninguna activación verdadera y que todas las detectadas sean falsas, hasta $100 \%$ en caso de que todas las contracciones voluntarias sean detectadas y que no exista ninguna falsa detección.

La métrica HFD es especialmente útil para interfaces asincrónicas tipo switch (Schlögl et al. 2007, Mason et al. 2006) donde hay un único evento o estado a detectar (contracción) respecto de un estado de "no control" (el estado de reposo), que es mucho más probable que el estado de activación. La misma se centra en las transiciones del estado de reposo al de contracción.

Como difícilmente el sujeto presionó la tecla exactamente al iniciar la contracción y además los algoritmos de detección tienen retrasos temporales inherentes a los mismos, se determina una ventana temporal en torno a la marca de referencia donde es correcta la detección de una contracción (ver Figura 5-10) (Mason et al. 2006). Si 
ninguna transición es detectada en dicha ventana, se considera que la contracción no fue detectada. Las contracciones detectadas fuera de dichas ventanas no guardan relación con ninguna contracción voluntaria realizada por el sujeto y son consideradas falsas detecciones.

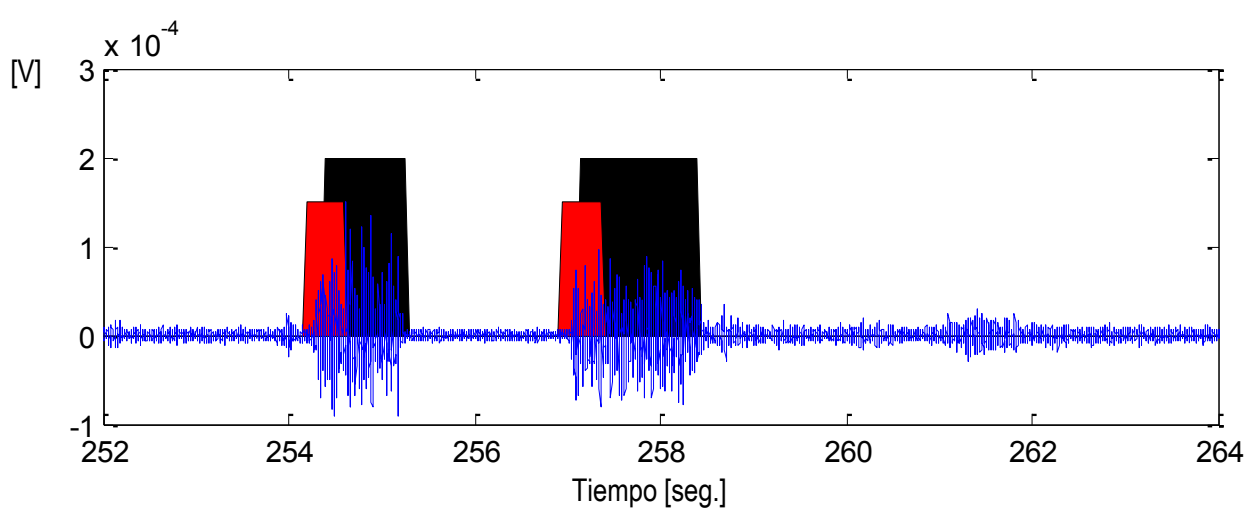

Figura 5-10: En azul: señal de EMG del cigomático mayor. En negro: segmento de tiempo indicado por el sujeto como una contracción voluntaria. En rojo: ventana de tiempo donde se espera que el algoritmo detecte la contracción.

\subsubsection{Resultados}

En la Tabla 5-1 se presenta una comparación de las distintas técnicas de detección de contracciones en términos de la HFD incluida la comparación con un umbral fijo calculado según (5-1) con $K=K_{U I I}=12$, donde la media y la desviación se tomaron sobre los primeros 10 segundos de cada registro, donde el sujeto mantuvo en reposo el músculo.

\begin{tabular}{cccccccc}
\hline Registro & Músculo & $\begin{array}{c}\text { Duración } \\
{[\mathbf{s}]}\end{array}$ & $\begin{array}{c}\text { SNR } \\
\text { [dB] }\end{array}$ & NT & $\begin{array}{c}\text { HFD [\%] } \\
\text { (Tecnica I) }\end{array}$ & $\begin{array}{c}\text { HFD [\%] } \\
\text { (Tecnica II) }\end{array}$ & $\begin{array}{c}\text { HFD [\%] } \\
\text { (Umbral fijo) }\end{array}$ \\
\hline $\mathbf{1}$ & $\mathrm{BBI}$ & 342 & 20 & 34 & 100 & 100 & 100 \\
$\mathbf{2}$ & $\mathrm{BBI}$ & 415 & 21 & 52 & 100 & 100 & 100 \\
$\mathbf{3}$ & $\mathrm{BBI}$ & 466 & 21 & 43 & 92 & 91 & 88 \\
$\mathbf{4}$ & $\mathrm{BBI}$ & 321 & 15 & 67 & 99 & 100 & 100 \\
$\mathbf{5}$ & FD & 320 & 15 & 43 & 95 & 100 & 93 \\
$\mathbf{6}$ & FD & 358 & 14 & 47 & 98 & 100 & 100 \\
$\mathbf{7}$ & FD & 176 & 17 & 38 & 100 & 97 & 46 \\
$\mathbf{8}$ & FD & 201 & 21 & 43 & 98 & 98 & 90 \\
\hline
\end{tabular}




\begin{tabular}{cccccccc}
\hline $\mathbf{9}$ & ZMD & 196 & 13 & 50 & 94 & 94 & 94 \\
10 & ZMD & 287 & 14 & 60 & 100 & 95 & 97 \\
11 & ZMD & 231 & 13 & 62 & 100 & 100 & 100 \\
12 & ZMD & 327 & 14 & 61 & 98 & 98 & 97 \\
Total & & 1h 0m 40s & 600 & 98 & 97 & 93 \\
\hline
\end{tabular}

Tabla 5-1: Comparación de ambas técnicas propuestas para la detección de contracciones, junto con la detección por umbral fijo aplicado a los 12 registros.

\subsubsection{Resultados con incremento de ruido simulado}

Para ilustrar el comportamiento de las distintas técnicas ante cambios en el nivel base se simularon, a partir de los registros reales, incrementos del ruido y la interferencia de $50 \mathrm{~Hz}$, por separado, a la mitad de la duración de cada registro. En el caso del ruido este se incrementa a $10 \mu V_{\mathrm{RMS}}$ mientras que la interferencia de $50 \mathrm{~Hz}$ se incrementa a $10 \mu V$ su amplitud.

La Tabla 5-1 muestra como ambas técnicas propuestas aportan en general mejores resultados que con la utilización de un umbral fijo. La diferencia entre las técnicas propuestas y la comparación con umbrales fijos se vuelve evidente en los casos donde hay un marcado cambio de las condiciones de ruido base, por ejemplo en el registro $\mathrm{N}^{\circ} 7$ que se observa en la Figura 5-7. La adición de ruido simulado muestra la robustez de ambas técnicas ante cambios en el nivel base en contraste con el uso de un umbral fijo.

Entre las técnicas desarrolladas la elección de una sobre la otra no muestra grandes diferencias para señales de EMG con niveles de ruido bajos a moderados (SNR > 9db). Sin embargo para SNR menores a 9dB la balanza se inclina fuertemente hacia la Técnica II que da buenos resultados hasta SNR $>6 \mathrm{db}$. Esto se puede observar para un segmento del registro $\mathrm{N}^{\circ} 12$ al que se le adicionó ruido $(S N R \approx 8 \mathrm{db})$ la Técnica I produce un umbral demasiado alto y solo identifica correctamente 8 de 15 contracciones (HFD=53\%), mientras que la Técnica II (ver Figura 5-11) identifica 14 (HFD=93\%). Claramente esta última resulta mucho más robusta al ruido.

\begin{tabular}{llllllllll}
\hline Registro & \multicolumn{1}{l}{ incremento del ruido } & \multicolumn{7}{l}{ Incremento de la interferencia } \\
\cline { 2 - 8 } & $\begin{array}{l}\text { HFD [\%] } \\
\text { (Tecnica I) }\end{array}$ & $\begin{array}{l}\text { HFD } \\
\text { (Tecnica II) }\end{array}$ & $\begin{array}{l}\text { HFD } \\
\text { (Umbral fijo) }\end{array}$ & $\begin{array}{l}\text { HFD } \\
\text { (Tecnica I) }\end{array}$ & $\begin{array}{l}\text { HFD } \\
\text { (Tecnica II) }\end{array}$ & $\begin{array}{l}\text { HFD [\%] } \\
\text { (Umbral fijo) }\end{array}$ \\
\hline $\mathbf{1}$ & 100 & 100 & 47 & 100 & 100 & 41 \\
\hline $\mathbf{2}$ & 100 & 100 & 52 & 100 & 100 & 44 \\
\hline
\end{tabular}


Capítulo 5 - Procesamiento digital de EMG para Dispositivos de Ayuda

\begin{tabular}{lllllll}
\hline $\mathbf{3}$ & 89 & 91 & 40 & 89 & 91 & 41 \\
$\mathbf{4}$ & 99 & 100 & 65 & 99 & 100 & 74 \\
$\mathbf{5}$ & 100 & 100 & 70 & 100 & 98 & 70 \\
$\mathbf{6}$ & 98 & 100 & 85 & 98 & 100 & 51 \\
$\mathbf{7}$ & 100 & 97 & 46 & 100 & 90 & 46 \\
$\mathbf{8}$ & 100 & 98 & 58 & 100 & 100 & 53 \\
$\mathbf{9}$ & 96 & 94 & 82 & 96 & 94 & 65 \\
10 & 98 & 97 & 58 & 98 & 97 & 53 \\
11 & 100 & 100 & 98 & 100 & 100 & 87 \\
\hline 12 & 97 & 98 & 94 & 92 & 98 & 88 \\
\hline Total & 98 & 98 & 67 & 97 & 98 & 60 \\
\hline
\end{tabular}

Tabla 5-2: Comparación de ambas técnicas propuestas con la detección por umbral fijo ante incrementos de ruido aleatorio e interferencia de $50 \mathrm{~Hz}$. A cada registro se le sumo una señal espuria a la mitad de duración del mismo. En el caso del ruido este es de $10 \mu V_{R M S}$ y en el caso de la interferencia se sumó una sinusoide de $50 \mathrm{~Hz}$ y $10 \mu \mathrm{V}$ de amplitud.
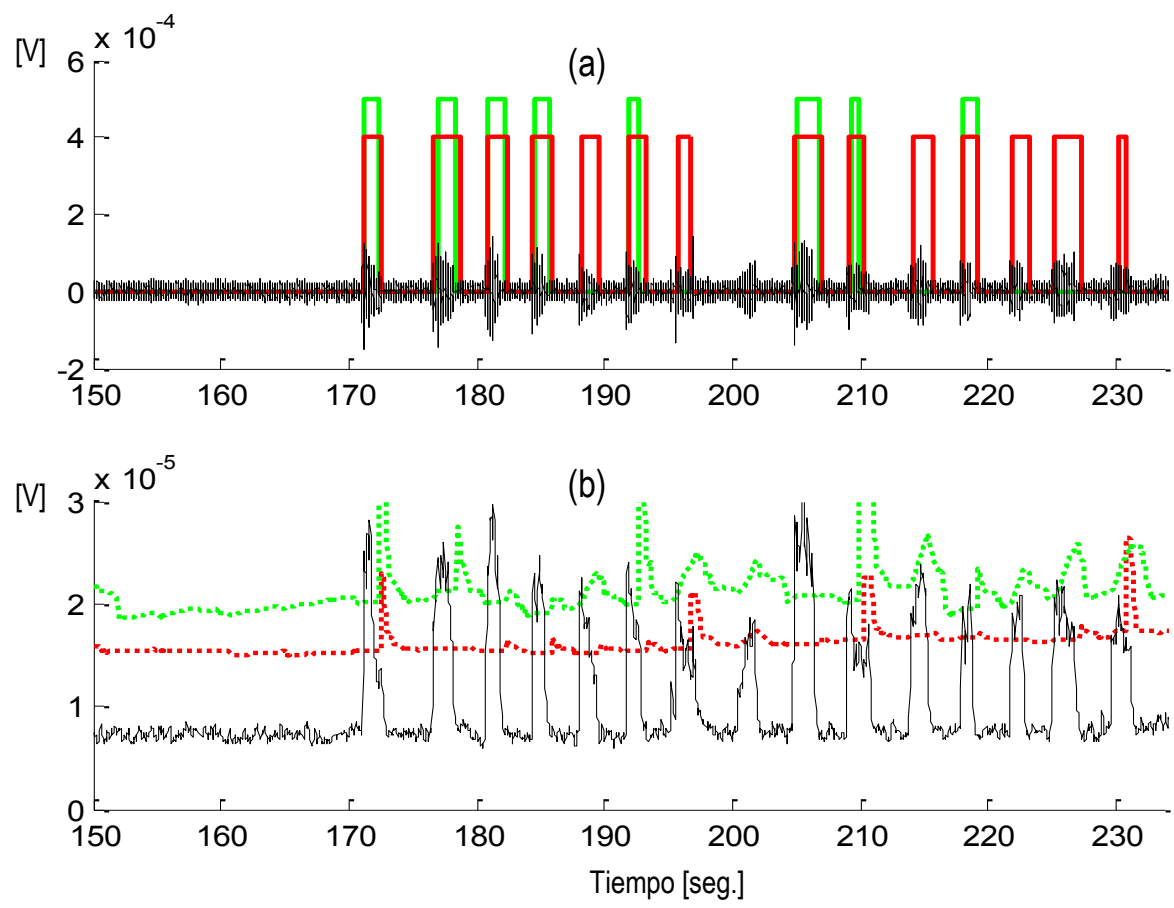

Figura 5-11: Segmento del registro $\mathrm{N}^{\circ} 12$ al que se le adicionó ruido aleatorio, resultando en una $S N R \approx 8 \mathrm{~dB}$. (a) Señal de EMG cruda $x[k]$ y salidas binarias salida $[k]$ (verde: Técnica I, rojo: Técnica II) (b) característica $y[k]$ y umbral $[k]$ (verde: Técnica I, rojo: Técnica II). 


\subsubsection{Prueba de los algoritmos sobre señales de EMG de persona con ELA}

Sobre un registro de señales de EMG del músculo ZMI de una persona con ELA en estado avanzado, se aplicaron ambas técnicas. Dado que se disponían de marcas temporales de las contracciones voluntarias realizadas fue posible evaluar el desempeño de los algoritmos.

Las señales poseían características muy diferentes de las anteriormente evaluadas (ver Figura 5-5), dado que la persona con ELA podía controlar muy pocas unidades motoras, por lo que las señales resultan sumamente débiles. Además no tenía experiencia previa ni entrenamiento en el uso del músculo ZMI para el control de interfaces basadas en EMG.

De las 24 contracciones voluntarias realizadas ambas técnicas detectaron el total de las mismas y también ambas tuvieron una falsa detección, resultando $H F D=96 \%$ y mostrando la robustez de las técnicas desarrolladas ante el cambio de usuario.

En la Figura 5-12 puede verse un segmento de dicho registro y el resultado de aplicar ambas técnicas de detección.
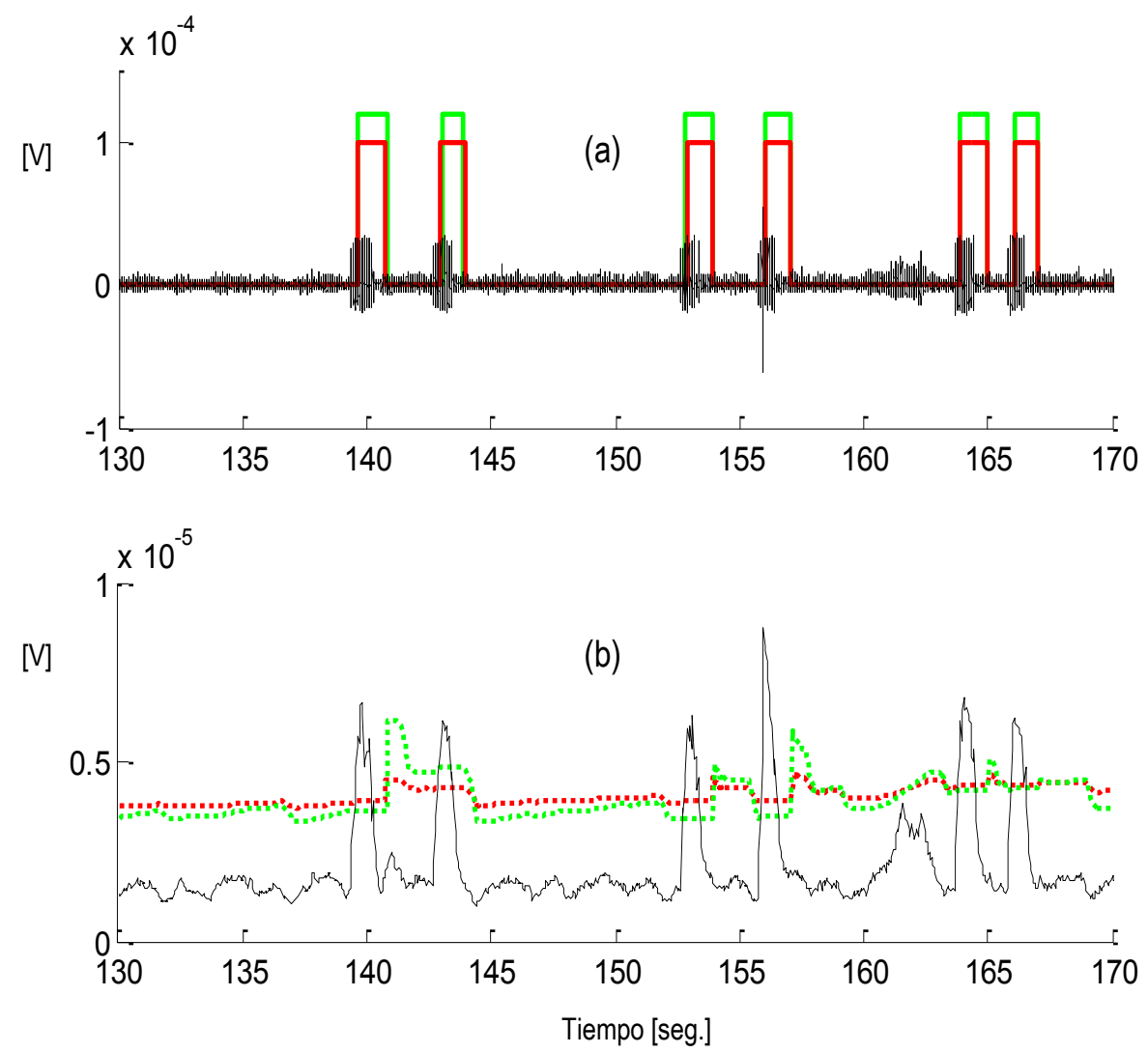

Figura 5-12: Segmento del registro sobre el ZMI de persona con ELA. (a) Señal de EMG cruda $x[k]$ y salidas binarias salida $[k]$ (verde: Técnica I, rojo: Técnica II) (b) característica $y[k]$ y umbral $[k]$ (verde: Técnica I, rojo: Técnica II). 


\subsection{Conclusiones}

En los DA controlados por EMG es fundamental un algoritmo que a partir de las muestras digitalizadas del EMG logre distinguir entre estados de reposo y contracción en tiempo real. La revisión bibliográfica muestra que solo una solución plantea la idea de un umbral móvil para la detección de contracciones en tiempo real (Williams y Kirsch 2008) mientras que el resto hace uso de umbrales estáticos. No obstante, dicha solución depende del conocimiento de la señal durante la contracción, lo cual requeriría entrenar el algoritmo cada vez que se coloquen los electrodos.

En este capítulo se propusieron dos técnicas que evitan el ajuste de los parámetros de procesamiento, calculando el umbral a partir de las condiciones del nivel base, como es usual en algunas técnicas para la detección del comienzo de la contracción. En las técnicas propuestas en este capítulo, el algoritmo está pensado para su ejecución en tiempo real y el objetivo es tener buena sensibilidad con el mínimo de detecciones espurias (alto HFD) sin ser tan crítica la exactitud temporal de la detección.

Las técnicas fueron ensayadas (y ajustadas) sobre registros reales de EMG de tres músculos distintos. Si bien no constituye una muestra significativa ya que todos los registros corresponden a la misma persona, los resultados son promisorios y muestran la aptitud de ambas técnicas para ser implementadas en un DA real. La prueba sobre la señal de la persona con ELA con los parámetros ajustados con primer el banco de registros muestra que es posible aplicar los algoritmos sin recalibrar los mismos.

El principal aspecto a resaltar es la robustez de las técnicas presentadas ante cambios del nivel base y su independencia de las características de la señal durante la contracción. Esto permite implementar interfaces que no requieran tediosas y repetitivas sesiones de ajuste, lo cual es muy ventajoso ya que se reducen los costos de mantenimiento y la capacitación de las personas que asisten al usuario del DA. Otro aspecto beneficioso de los algoritmos propuestos es su simplicidad, lo cual permite implementarlos en microcontroladores de bajo costo y con bajo consumo. 


\section{Capítulo 6}

\section{Implementación de un DA tipo switch}

En el presente capítulo se presenta la implementación de un Dispositivo de Ayuda tipo Switch, donde se integran diversos aspectos tratados en los capítulos previos de esta tesis.

El diseño del DA responde a criterios de practicidad, simplicidad, robustez, seguridad y versatilidad, cuyo cumplimiento requirió soluciones conjuntas de hardware y del software embebido. El dispositivo actúa emulando el click de un mouse de PC, conectándose a la misma a través del puerto USB, tomando la energía de éste para funcionar. Dado que se muestra como un mouse no requiere drivers ni software adicional. Esto, en conjunto con la implementación embebida de una de las técnicas de detección de contracciones propuestas en el Capítulo 5 , hacen al dispositivo muy simple de poner en funcionamiento, ya que no requiere calibración ni instalación de drivers: el dispositivo está listo para utilizar con solo colocar los electrodos sobre el músculo de interés y conectarlo al puerto USB. A pesar de estar conectado a una PC el dispositivo es seguro, ya que la electrónica asociada a los electrodos (lado paciente) transmite las señales y se alimenta en forma aislada

El dispositivo, además de constituir un DA, tiene como doble propósito ser una herramienta de investigación. Como DA presenta un perfil de mouse, pero también permite acceder en tiempo real a las señales adquiridas de EMG y otras señales internas del procesamiento. Para este último modo de funcionamiento se desarrollaron distintas aplicaciones para la PC que permiten visualizar las señales en tiempo real y registrarlas, así como para modificar parámetros de procesamiento y realizar experimentos.

El DA presentado se completa con un conjunto de aplicaciones "asistivas" que permiten el acceso a la PC mediante un único click. La oferta de este tipo de aplicaciones es muy vasta (teclados virtuales, navegadores, control domótico), muchas de ellas son gratuitas y ampliamente configurables. Estas aplicaciones, operadas mediante el DA propuesto, ofrecen al usuario una enorme versatilidad para la comunicación y el control de su entorno. 


\subsection{Introducción}

Un objetivo constantemente mencionado a lo largo de la tesis es conseguir DAs que resulten de utilidad a los usuarios, mejorando su calidad de vida. Si bien parece una obviedad decir que un DA debe ser de utilidad, hay muchos aspectos que en la práctica resultan obstáculos para el uso y su aceptación por parte de los usuarios. Es así como se ven muchas soluciones en el ámbito del laboratorio, con buenos resultados técnicos en términos de la performance objetiva que no logran constituirse en soluciones comerciales. En (Davies et al. 2010) se hace una revisión sobre DAs para el acceso a la PC de personas con parálisis cerebral, rescatándose que en la aceptación por parte del usuario, el confort, el esfuerzo involucrado y la simplicidad, son tan importantes como el desempeño objetivo del dispositivo. En (Graimann et al. 2010) se dice, respecto de las $\mathrm{BCl}$, que su éxito por fuera del laboratorio dependerá de su confiabilidad y de su aceptación por parte de los usuarios.

Teniendo en cuenta estos aspectos se planteó desarrollar un DA que consiste en un dispositivo tipo switch, cuyo uso está muy difundido ( véanse por ejemplo los sitios https:// www.ablenetinc.com/ technology/switches 0 https:// enablingdevices.com). Esto facilita su recomendación por parte de terapeutas, a la vez que existen numerosas aplicaciones desarrolladas para este tipo de dispositivos.

En cuanto a la elección del EMG respecto de señales de EEG como señal de control, esto corresponde a que estas señales poseen mejor relación señal ruido que las que se pueden obtener del EEG y es más sencilla y rápida la colocación de los electrodos. Además EMG se logran mayores velocidades.

A su vez, aún en las afecciones neurodegenerativas más graves como ELA, suele quedar alguna actividad residual en los músculos faciales antes de llegar a un estado de enclaustramiento. Aún sin un movimiento apreciable, es posible captar el EMG. En casos más graves, se puede evaluar el uso de interfaces híbridas (Leeb et al 2011, Müller-Putz 2011) que combinan EEG y EMG.

Los distintos temas tratados en la tesis se materializan en el dispositivo desarrollado de manera que este cumpla con las siguientes metas de diseño:

- Simplicidad de operación por parte del usuario y de instalación para quien lo asista: Esto se manifiesta en la construcción de un único sensor en contacto con el usuario, que concentra la electrónica de instrumentación de EMG. De esta manera el sensor se coloca fácilmente sobre la piel y se conecta con el resto del equipo por un cable plano flexible que no ofrece resistencia mecánica de importancia.

Se utilizó una implementación embebida de la Técnica I para la detección de contracciones que no requiere prácticamente ajustes ni calibración, así como en la manera en que se materializa la salida (ON/OFF) del switch, lo cual se realiza emulando el click del mouse. El equipo de hecho se muestra como un mouse USB, con lo cual no requiere instalar drivers adicionales en ningún sistema operativo, mientras que a través de dicho puerto obtiene la energía para funcionar, evitando el uso de baterías y su mantenimiento.

- Seguridad: El equipo posee aislación interna de manera de desvincular eléctricamente al usuario de la PC. Esta aislación cumple con los requisitos para equipos médicos, y aísla tanto la alimentación como la señal entre el usuario y el resto del equipamiento conectado a la PC.

- Robustez: Por el lado de la instrumentación, la realimentación de modo común se diseñó con una ganancia suficientemente alta de manera de eliminar la tensión de modo común a la frecuencia de red asegurando la estabilidad aun con las peores combinaciones de capacidades parásitas. Esto facilita la utilización del DA en ambientes hostiles en términos de EMI. 
El uso de electrodos preamplificados reduce la interferencia de modo diferencial por acoplamiento capacitivo de la red a los cables, mientras que el uso de cable plano reduce el área de inducción para la interferencia por campo magnético.

El procesamiento de la señal para detectar las contracciones es en sí mismo una solución diseñada para ser robusta.

- Versatilidad: La emulación de mouse le da la posibilidad de ser usado con cualquier aplicación que requiera un click para operar. Permite el desarrollo de aplicaciones ad-hoc para los usuarios por parte de terceros.

\subsubsection{Proceso de desarrollo y métodos}

El dispositivo desarrollado surge como resultado de un proceso con sucesivas iteraciones en las cuales se implementaron diversos prototipos que fueron incorporando paulatinamente las funcionales mencionadas.

Un factor clave durante el transcurso de dicho proceso fue la colaboración voluntaria de una mujer con ELA que probó y utilizó distintos prototipos. La voluntaria conservaba algún grado de control voluntario sobre el movimiento de los ojos y un gesto con la comisura de los labios. Se evaluó utilizar el gesto con la comisura de los labios (realizado por la contracción del músculo zigomático mayor) para controlar un switch y de esta manera un teclado en la pantalla de una PC portátil. A la vez que fue una motivación importante para la búsqueda de un dispositivo funcional con las características mencionadas, permitió adquirir un conocimiento práctico acerca de distintos aspectos operativos y de las condiciones del contexto donde pueden llegar a funcionar estos dispositivos.

Los primeros prototipos fueron básicamente adquisidores de señales biopotenciales de propósitos generales con conexión a PC, donde se puso principal empeño en la instrumentación de bajo ruido de las señales biopotenciales. Se desarrollaron aplicaciones de PC para almacenar las señales y visualizarlas en tiempo real. Se ensayaron fuera de línea, sobre distintos registros almacenados, distintas aproximaciones a lo que sería el procesamiento de las señales de EMG para la detección de contracciones utilizando el software Matlab.

En estas primeras etapas se ganó conocimiento práctico sobre la instrumentación del EMG, se implementaron y compararon entre sí distintas configuraciones de electrodos y se concluyó que era indispensable la utilización de electrodos activos para rechazar efectivamente distintas fuentes de EMI, no solo de la red, sino de artefactos médicos o domésticos que puede haber en el entorno. Se observó también que los electrodos secos ofrecen más estabilidad en sesiones extensas, pero requieren un soporte mecánico adicional, mientras que los electrodos húmedos descartables facilitan la fijación pero con el tiempo puede secarse el gel electrolítico y perder la adhesión.

También se pudo verificar la variabilidad que presentan las señales de EMG, entre músculos, entre individuos, ante cambios sutiles en la ubicación de los electrodos, y principalmente se observó lo débiles que pueden ser las señales producidas por pequeños músculos faciales en personas con ELA.

Así surgió una primera versión de la Técnica I propuesta en el Capítulo 5 a fin de evitar incómodos protocolos de entrenamiento o frecuentes ajustes manuales. Posteriormente se implementó en un software de $\mathrm{PC}$ programado en C\#, que recibía las muestras crudas de EMG y detectaba las contracciones en tiempo real (no estricto, ya que corre sobre Windows). La variedad de aplicaciones para facilitar la accesibilidad a la PC y aumentar las capacidades de comunicación, como por ejemplo el software Plaphoons del proyecto Fressa, llevó a la conclusión de que la salida más útil para el dispositivo era la emulación del click. Esto se implementó en la aplicación de PC utilizando las funciones del API de Windows. Posteriormente también se agregaron señales sonoras a la aplicación para realimentar al usuario los instantes en que el 
procesamiento detecta la contracción. Según se pudo comprobar dicha realimentación permite un control del DA con menos esfuerzo en la contracción.

En la Figura 6-1 se muestra un diagrama en bloques que ejemplifica los bloques más importantes del dispositivo y su utilización para controlar un teclado por barrido.

En la Figura 6-2, se muestra un esquema simplificado del hardware del DA presentado, donde se pueden ver los distintos bloques que lo componen. Se puede observar que el mismo está compuesto por dos placas, una es el sensor de EMG, que contiene los electrodos, junto con el $A B$ y la realimentación de modo común. La otra placa incluye el ADC, el dispositivo que implementa la barrera de aislación para la alimentación y las señales del bus SPI, así como el microcontrolador que implementa la detección de contracciones y la comunicación USB. Esta última placa va dentro de un gabinete plástico, del cual sale el cable plano que conecta al sensor de EMG y el cable USB que comunica con la PC (ver Figura 6-3)

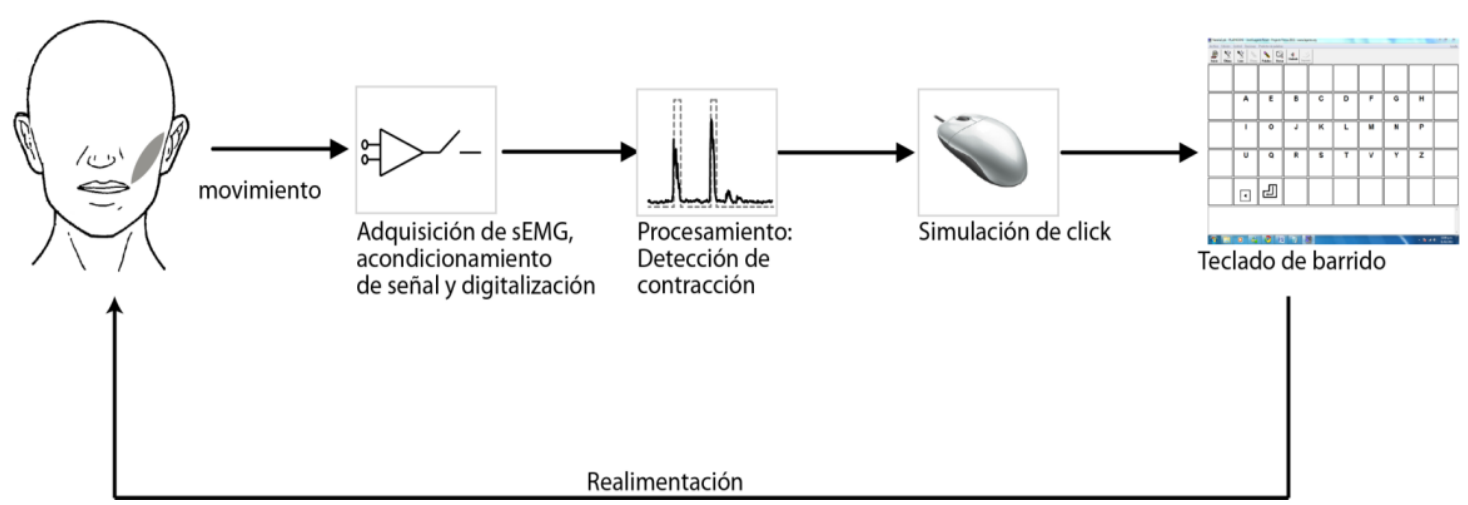

Figura 6-1: Esquema que resume la estrategia para el uso del Dispositivo de Ayuda desarrollado

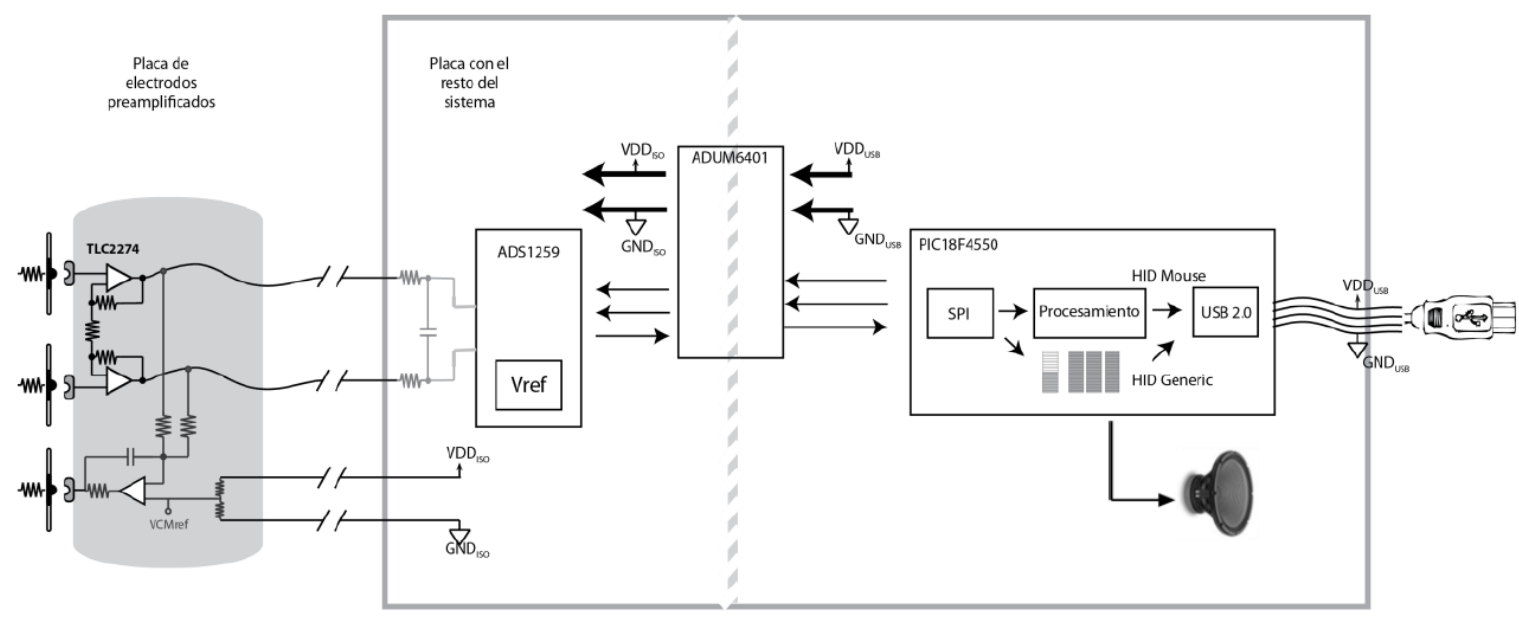

Figura 6-2: Circuito simplificado del DA desarrollado. 


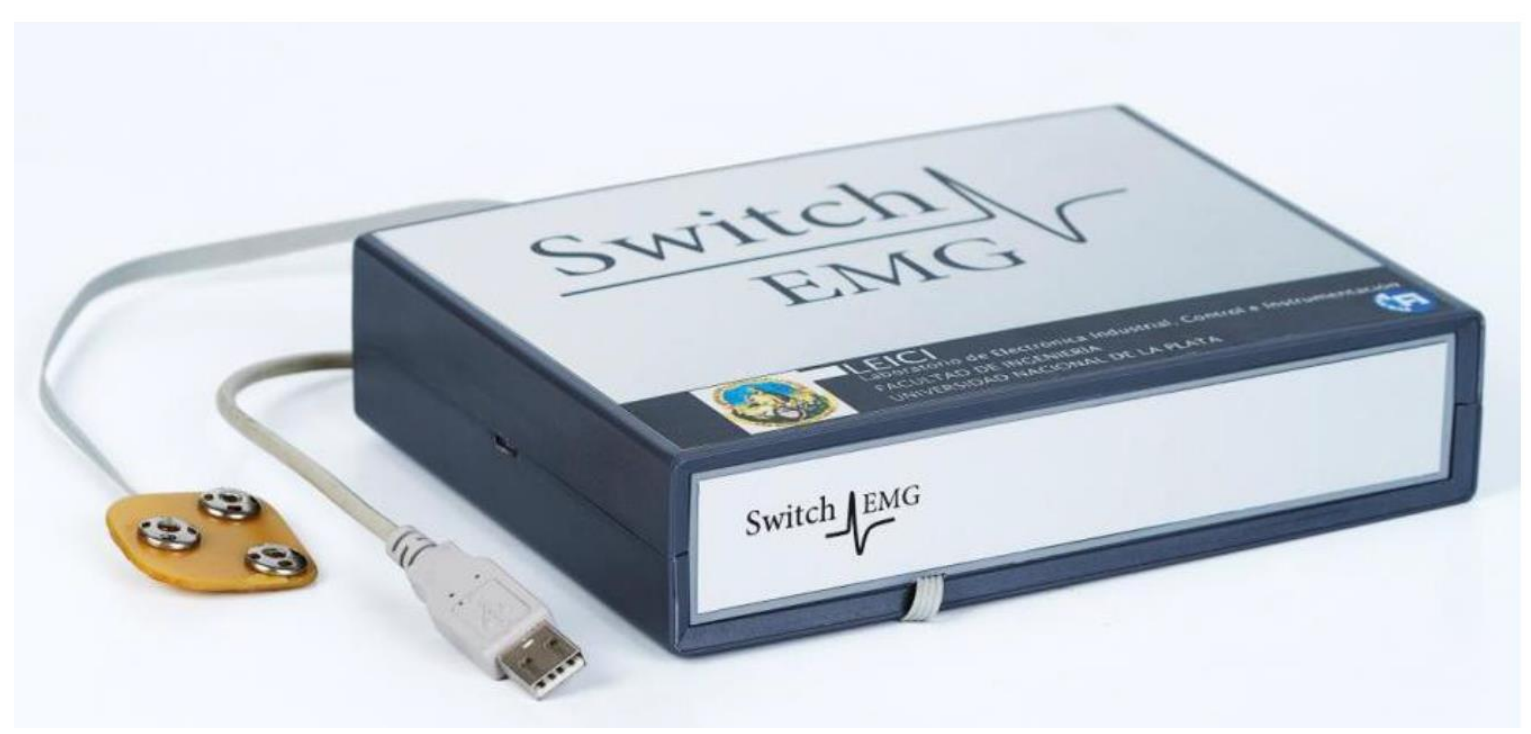

Figura 6-3: Prototipo del Dispositivo de Ayuda desarrollado.

\subsection{Hardware del Dispositivo de Ayuda}

\subsubsection{Sensor de EMG con alto rechazo de EMI}

Se confeccionó una placa de circuito impreso (mencionada en los Capítulos 3 y 4 ) a la que se conectan los dos electrodos de medida de la señal de EMG y uno extra para la realimentación de modo común. El circuito completo puede observarse en la Figura 6-4.

En la cara superior se encuentran los componentes de montaje superficial que conforman un amplificador de instrumentación (INA) completamente diferencial de ganancia $G_{D D}=10 \mathrm{~V} / \mathrm{V}$. Este está formado por dos de los cuatro amplificadores operacionales de bajo ruido del TLC2274 de Texas Instruments. El INA actúa como Amplificador de biopotenciales ( $\mathrm{AB}$ ) acoplado en continua con un rango de entrada de $\pm 250 \mathrm{mV}$ y un piso de ruido en el ancho de banda de interés $(30 \mathrm{~Hz}-250 \mathrm{~Hz})$ de $0,56 \mu \mathrm{V}_{\mathrm{RMS}}$. Esta configuración de electrodos activos preamplificados incrementa el rechazo de interferencia electromagnética (EMI), favorece el CMRR por la alta impedancia que presenta el amplificador y permite la utilización de cables flexibles, ya que no se requiere blindaje electromagnético. En este caso se utilizó cable plano de 4 conductores, un par para la señal diferencial amplificada y otro par para la alimentación del preamplificador.

Un tercer amplificador operacional es utilizado para conformar un lazo de realimentación de modo común (circuito DRL) que fija el modo común en 2,5V mientras que aumenta el CMRR con una muy alta ganancia a la frecuencia de red ( $56 \mathrm{~dB})$, manteniendo buenos márgenes de estabilidad según el análisis llevado a cabo en el Capítulo 4. El hecho de que el electrodo de realimentación de modo común se encuentre también sobre la misma placa facilita la colocación del DA en su utilización diaria.

Los terminales del circuito vinculados a los electrodos están soldados a conectores de acero inoxidable dispuestos en la cara opuesta de la placa. Estos conectores tienen una doble función, son compatibles con electrodos húmedos descartables de $\mathrm{Ag} / \mathrm{AgCl}$ cuyo adhesivo puede proveer la fijación mecánica a la piel. 
Pero también pueden apoyarse directamente sobre la piel y funcionar como electrodos secos, debiéndose fijarse el sensor al usuario por algún medio de sujeción.

\subsubsection{Conversión $A D$ de alta resolución}

Como fue mencionado, la salida de $A B$ se conecta a la entrada del $A D C$ por intermedio de un simple filtro pasabajos diferencial de primer orden que actúa como filtro anti-aliasing. El ADC utilizado es el ADS1259 de Texas Instruments, de tecnología sigma-delta. Este dispositivo posee una referencia interna de 2,5V, entrada diferencial de tensión, con un rango de entrada de $\pm 2,5 \mathrm{~V}$ en modo diferencial y de $0 \mathrm{~V}$ a $5 \mathrm{~V}$ en modo común. La salida digital es a través de una interfaz serie sincrónica (SPI), entregando cada muestra en un código de 24 bits. El conversor posee un ruido propio referido a su entrada, que para la configuración establecida (1200SPS, filtro sinc2) es de $14 \mu \mathrm{V}_{\mathrm{PP}}$. Referido a los electrodos, este ruido es de $1,4 \mu \mathrm{V}_{\mathrm{PP}}$, la mitad del ruido propio del $A B$ y aproximadamente 5 veces menor al producido en la interfaz electrodo-piel.

\subsubsection{Seguridad eléctrica}

Es de importancia notar que el usuario del DA estará en contacto con electrodos y el equipo a su vez estará conectado al puerto USB de una PC. De no mediar algún tipo de aislación eléctrica se estaría exponiendo al usuario a riesgos sumamente graves, dado las corrientes de fuga a tierra que circularían por el mismo podrían ocasionarle algún perjuicio, y ante una sola falla este podría quedar vinculado directamente a la tensión de red.

Por ello, se debe aislar eléctricamente las partes que entran en contacto con el usuario (denomina "parte aplicada" por la norma IEC 60601-1) de cualquier potencial que pueda generar una corriente de fuga a tierra o a otra parte accesible del equipo superior a las permitidas por la norma IEC 60601-1. Esto significa que el $A B$ y los electrodos deben aislarse del potencial del puerto USB, tanto los caminos de la señal como de la alimentación. Esto, que suele hacerse por medios separados, se realizó con un único circuito integrado, el ADUM6401 de Analog Devices que, cumpliendo los requisitos de aislación y distancias de separación de la norma IEC 60601-1, implementa la aislación de la fuente de alimentación de 5V del AB y del $A D C$, así como de las líneas digitales de la interfaz SPI a través de la cual se transfieren los datos del $A D C$ al microcontrolador. 


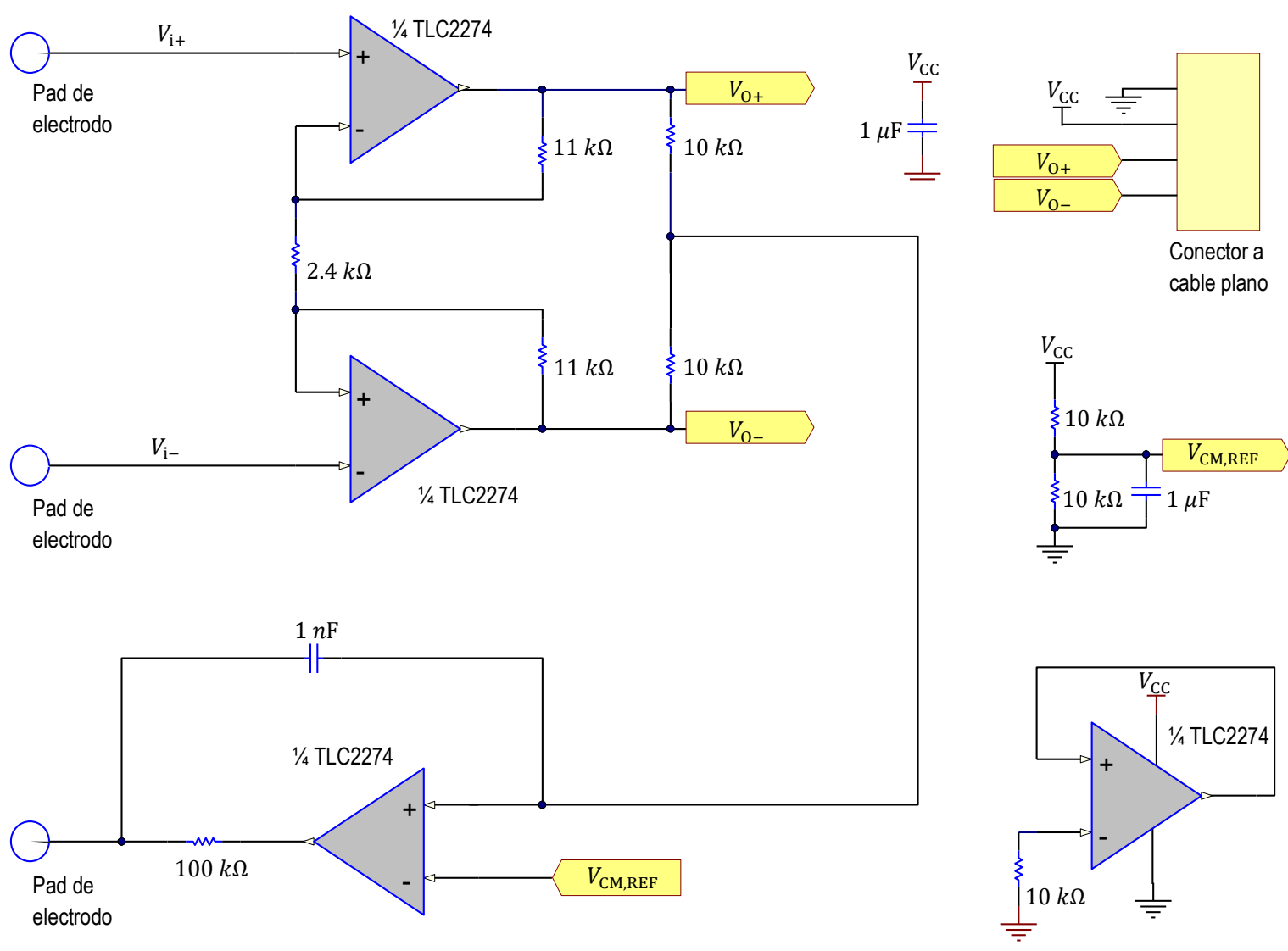

Figura 6-4: Circuito completo del electrodo activo desarrollado, que incluye prácticamente todas las etapas analógicas que intervienen en la instrumentación de EMG.

\subsubsection{Controlador embebido con interfaz USB}

Como controlador se utilizó el microcontrolador de 8 bits PIC18F4550, cuya principal característica es que posee un periférico USB Device configurable. Esto permitió programar el equipo como una Interface HID tipo mouse, a la vez que una segunda interface HID genérica (Axelson 2005) permite utilizarlo como dispositivo de investigación, accediendo a las señales biopotenciales adquiridas.

\subsubsection{Realimentación sonora}

En la experiencia con prototipos previamente construidos, se notaron las ventajas que da la devolución de una realimentación al usuario. En un principio esta se agregó al software de PC que realizaba al detección de las contracciones en tiempo real, al embeber el procesamiento en el equipo se incorporó un zumbador (buzzer), controlado por una salida digital del microcontrolador, que entrega una señal sonora al detectarse la contracción y emular el click.

\subsubsection{Alimentación de energía}

La energía para el funcionamiento del equipo se obtiene del puerto USB de la PC, que entrega $5 \mathrm{~V}$ nominales y garantiza hasta $100 \mathrm{~mA}$ (el consumo total del equipo no supera los $75 \mathrm{~mA}$ ), pudiendo suministrar hasta $500 \mathrm{~mA}$ si la PC lo soporta. Todos los circuitos integrados utilizados funcionan con $5 \mathrm{~V}$ de alimentación, por 
lo que no fue necesario adaptar niveles de tensión. El ADUM6401, previamente mencionado, implementa la aislación de dicha fuente, entregando también 5V, pero aislados del potencial del USB de la PC.

\subsection{Firmware del Dispositivo de Ayuda}

El firmware del dispositivo está programado en lenguaje $C$, en forma modular y en distintos niveles de abstracción.

Los módulos de bajo nivel, controlan los periféricos utilizados, como por ejemplo:

- Puerto SPI

- Memoria EEPROM (almacenamiento no volátil de parámetros de configuración)

- USB Device (stack de código abierto provisto por Microchip al que se le realizaron modificaciones)

Así mismo, en un nivel más alto se implementaron los módulos que hacen uso de los de menor nivel.

- Módulo para el manejo del conversor ADS1259 (Utiliza el puerto SPI)

- Módulo de comunicación secundaria con la PC (accede a la memoria EEPROM y al stack USB para el envío y recepción de datos)

- Módulo de procesamiento de la señal de EMG y emulación de click (accede al módulo de comunicación con la PC para almacenar los datos a transferir si esto fue configurado, a la memoria EEPROM y al stack USB para la emulación del click)

Al conectar el dispositivo al USB y energizarse, se ejecuta una rutina de inicialización donde se configuran los distintos periféricos, a la vez que se espera que el host USB (usualmente una PC) detecte el dispositivo y establezca comunicación en un proceso denominado "enumeración" (Axelson 2005). Una vez realizado lo anterior el programa entra en un bucle infinito que invoca secuencialmente a las siguientes tareas:

- Detección de contracciones y emulación de mouse

- Manejo del protocolo de comunicación secundaria con la PC

- Manejo de transferencias USB de bajo nivel

A continuación se describen las dos primeras dado que la última es provista por Microchip y solo debe llamarse a la función correspondiente en cada iteración del bucle.

\subsubsection{Detección de contracciones y emulación de mouse}

Esta tarea Implementa el procesamiento de las muestras de EMG haciendo uso de la Técnica I propuesta en el Capítulo 5, pero con una leve diferencia respecto de lo presentado, donde el umbral se calculaba según

$$
\text { umbral }[k]=K_{\mathrm{UI}} \text { base }[k-1]
$$

En la implementación presentada se utiliza una banda de histéresis, definiendo dos constantes distintas $K_{\mathrm{UI}, \mathrm{R}}$ y $K_{\mathrm{UI}, \mathrm{C}}$, según se esté en estado de reposo o contracción, respectivamente. Esto permitió probar dicha estrategia para el rechazo de detecciones espurias o rebotes. Por lo tanto, la diferencia radica en que en la presente implementación el cálculo del umbral resulta: 


$$
\text { umbral }[k]= \begin{cases}K_{\mathrm{UI}, \mathrm{R}} \cdot \text { base }[k-1], & \text { en reposo } \\ K_{\mathrm{UI}, \mathrm{C}} \cdot \text { base }[k-1], & \text { encontracción } \\ & \left(K_{\mathrm{UI}, \mathrm{R}}>K_{\mathrm{UI}, \mathrm{C}}\right)\end{cases}
$$

Al detectarse una contracción, se prepara la interfaz USB para el envío de un click. Esto se realiza a través de funciones del API provisto por Microchip para el envío de datos a través de interfaces USB-HID.

Cabe aclarar que esta tarea no se comunica directamente con el ADC (conectado al puerto SPI del microcontrolador) sino que al finalizar una nueva conversión es el ADC el que interrumpe el flujo normal de ejecución y una rutina de interrupción se encarga de leer la nueva muestra y ponerla a disposición en memoria, además de indicarle mediante una variable bandera a la tarea de procesamiento que efectivamente hay una nueva muestra.

Por otra parte, si desde la PC se indicó mediante el comando apropiado que se desea tener acceso a las señales internas del procesamiento, durante el mismo se almacenaran las señales indicadas en buffers para su posterior transmisión a la PC.

\subsubsection{Comunicación secundaria con la PC}

Al conectar el equipo a un host USB, en general una PC, se procede a la "enumeración" del mismo, esto es un proceso de reconocimiento del dispositivo por parte del host, donde el primero transfiere unas estructuras de datos de configuración denominadas descriptores. Es por medio de los descriptores donde el host conoce el consumo de energía que demandará el dispositivo y el ancho de banda que debe reservar para la comunicación (Axelson 2005) esto siempre y cuando se disponga de dichos recursos, ya que puede haber otros dispositivos enumerados previamente.

La programación del dispositivo para funcionar como mouse o como dispositivo HID genérico se resuelve cargando los valores adecuados en los descriptores de interfaz. En este caso habrá dos interfaces.

La transmisión de datos en ambos sentidos se resuelve a nivel de "reportes HID", los cuales no son otra cosa que arreglos o buffers de una cantidad preestablecida de bytes, determinada en los descriptores. Estos reportes se ubican en una zona especial de la memoria RAM, a la cual tiene acceso directo el módulo USB del microcontrolador sin intervención del microprocesador.

Los reportes se transfieren a través de canales lógicos denominados pipes (tuberías) que están representados por un endpoint (un concepto similar al puerto de una comunicación TCP o UDP).

Cada endpoint a utilizar se configura mediante un descriptor individual, en el cual se establece el tipo de transferencia que permite (para los dispositivos HID se utilizan transferencias tipo interrupt que aseguran integridad de datos y ancho de banda) así como el sentido de la comunicación (IN: del device al host / OUT: del host al device).

La enumeración y la administración de la comunicación está resuelta por una API provista por Microchip, por lo que no se requiere por parte de la programación más que el llamado a una función de inicialización y la invocación periódica a una máquina de estados que realiza la gestión de bajo nivel de la comunicación (hay otro modo manejado por interrupciones que no requiere el llamado periódico a esta función). En la programación se deberán armar los descriptores, los cuales en lenguaje $C$ son arreglos constantes de bytes almacenados en memoria de programa. 
El API de Microchip provee las interfaces correspondientes para transmitir o recibir reportes a través de los distintos endpoints configurados. Para transferir un reporte solo es necesario pasarle a una función del API un puntero al mismo (debe estar en el espacio de memoria RAM accesible al hardware USB), el número de endpioint asociado y la cantidad de bytes a transferir. En el caso de esperar la llegada de un reporte, se le pasará a una función análoga el puntero a donde se debe guardar el reporte recibido, el endpoint y el tamaño esperado del reporte. En ambos casos puede chequearse si se completó la transferencia o si esta sigue a la espera.

El funcionamiento como mouse exige una interfaz HID con un endpoint IN, ya que la comunicación es unidireccional. El click se simula cambiando el valor a un bit particular en un reporte de 3 Bytes, que luego es enviado a través del canal asociado a dicho endpoint.

Mientras que para la interfaz HID genérica se utilizan uno IN y otro OUT para establecer un canal bidireccional. Dado que se utilizan transferencias de tipo interrupt, se podrán transmitir hasta 64 KBytes por segundo, lo cual es más que suficiente para los 3,6 KBytes por segundo requeridos para transmitir la señal cruda de EMG, digitalizada con 24 bits y muestreada a 1200 muestras por segundo. Esto permite no solo transferir la señal cruda sino también otras señales internas del procesamiento.

Toda la comunicación realizada a través de esta interfaz secundaria sigue un protocolo de más alto nivel, de tipo maestro-esclavo, el cual fue implementado en otros dispositivos de instrumentación desarrollados en el LEICl (Guerrero et al. 2014) y permite a las aplicaciones que corren en la PC obtener información del dispositivo como la cantidad de canales o la frecuencia de muestreo, a la vez que permite configurarlo y obtener las señales digitalizadas.

\subsection{Aplicaciones secundarias desarrolladas para PC}

\subsubsection{Software para manipular parámetros del procesamiento}

Como se explicó en el Capítulo 5, las técnicas desarrolladas no requieren calibración ni ajuste de parámetros. No obstante, ocurre que ante una disminución sustancial de la SNR, particularmente por debajo de los $9 \mathrm{~dB}$, el umbral calculado por la Técnica I resulta muy elevado para detectar las contracciones.

Ante estos casos extremos puede resultar de utilidad variar el factor de multiplicación del nivel base $K_{\mathrm{UI}}$ (en la implementación presentada son dos: $K_{\mathrm{UI}, \mathrm{R}}$ y $K_{\mathrm{UI}, \mathrm{C}}$ ) por ese motivo se realizó una aplicación que

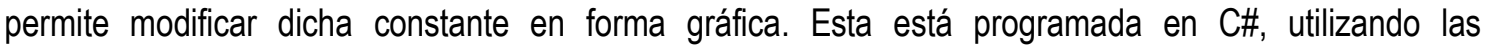
herramientas gráficas que brinda el entorno Visual Studio. Para la comunicación USB-HID se modificó una librería de código abierto la cual está basada en un envoltorio (wrapper) para .NET de las funciones que provee el API de Windows.

La aplicación se comunica con el DA a través de la interfaz HID secundaria y lo configura para transmitir la característica $y[\cdot]$ y el nivel base base ['] calculados en tiempo real por la tarea de procesamiento del DA. La aplicación presenta en pantalla la característica relativa al nivel base, es decir que grafica una nueva señal $z[k]=y[k] /$ base $[k-1]$, por lo que los umbrales de comparación resultan directamente las constantes $K_{\mathrm{UI}, \mathrm{R}}$ y $K_{\mathrm{UI}, \mathrm{C}}$. El operador no debe preocuparse por los valores de las constantes ya que el ajuste se realiza arrastrando dos líneas horizontales graficadas sobre el gráfico de $z[k]$ (Ver Figura 3-1).

Cada vez que se modifica el valor de alguno de dichos parámetros se envía un comando al DA a través de la interfaz secundaria. Dicho comando actualiza los valores utilizados por la tarea de procesamiento y los almacena en la memoria EEPROM. 


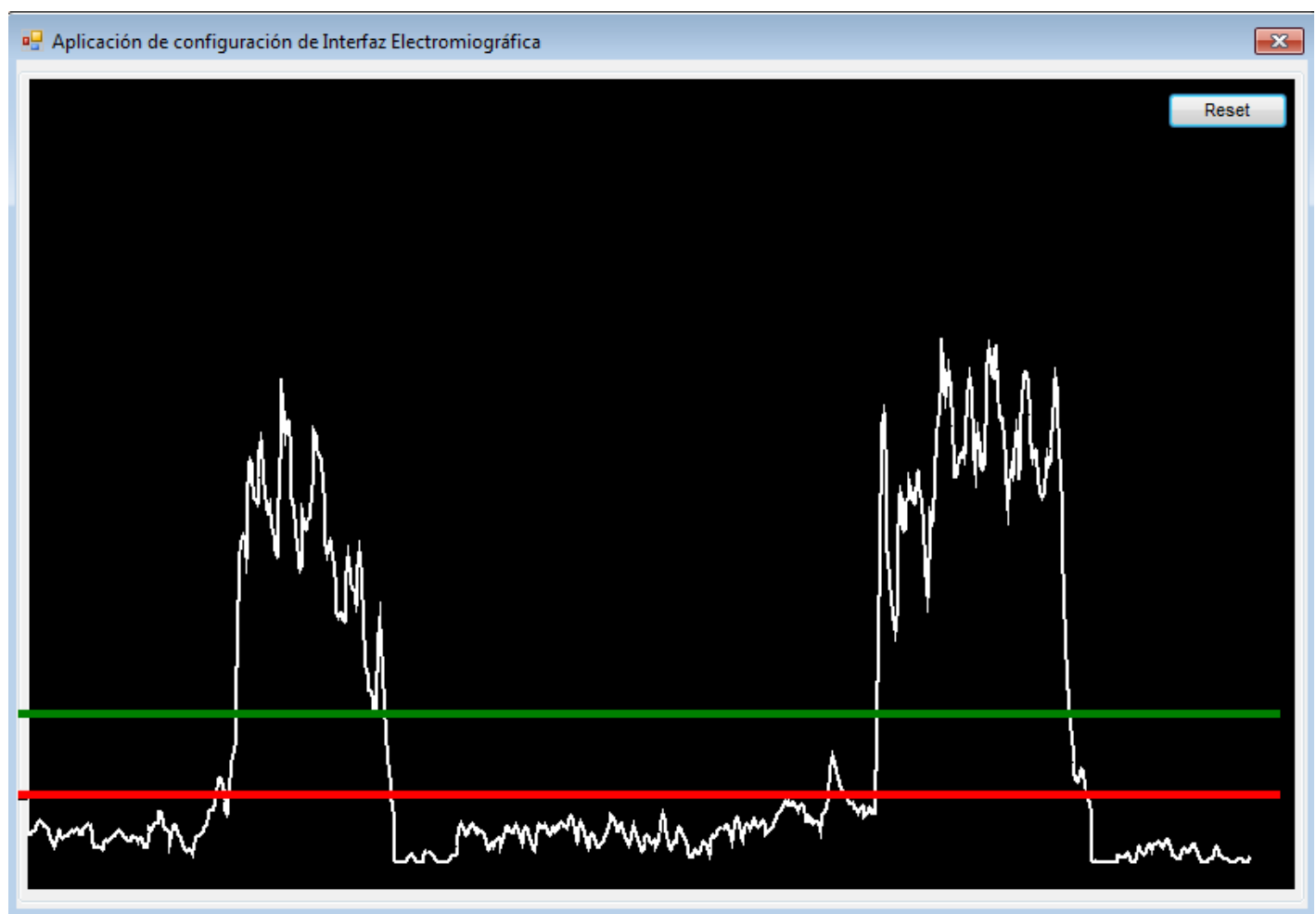

Figura 6-5: Captura de pantalla de la aplicación para modificar los parámetros $K_{\mathrm{UI}, \mathrm{R}}$ y $K_{\mathrm{UI}, \mathrm{C}}$. En blanco se ve la gráfica de la señalnormalizada $z[k]=$ $y[k] /$ base $[k-1]$, en verde $K_{\mathrm{UI}, \mathrm{R}}$ y en rojo $K_{\mathrm{UI}, \mathrm{C}}$. Los valores de estos parámetros se modifican arrastrando y soltando las líneas horizontales.

\subsubsection{Aplicaciones para visualización y registro de señales internas del procesamiento}

Con la misma base de la aplicación anterior se realizaron otras que permiten ver distintas señales sin modificar parámetros del procesamiento (ver Figura 6-6), a la vez que registran las señales recibidas en archivos.

Una aplicación en particular registra en un archivo aparte el tiempo en el que se presiona una tecla del teclado, con lo cual es posible introducir marcas temporales con lo que pueden experimentarse diversas técnicas de procesamiento y comparar los resultados, contrastando contra las marcas almacenadas. 


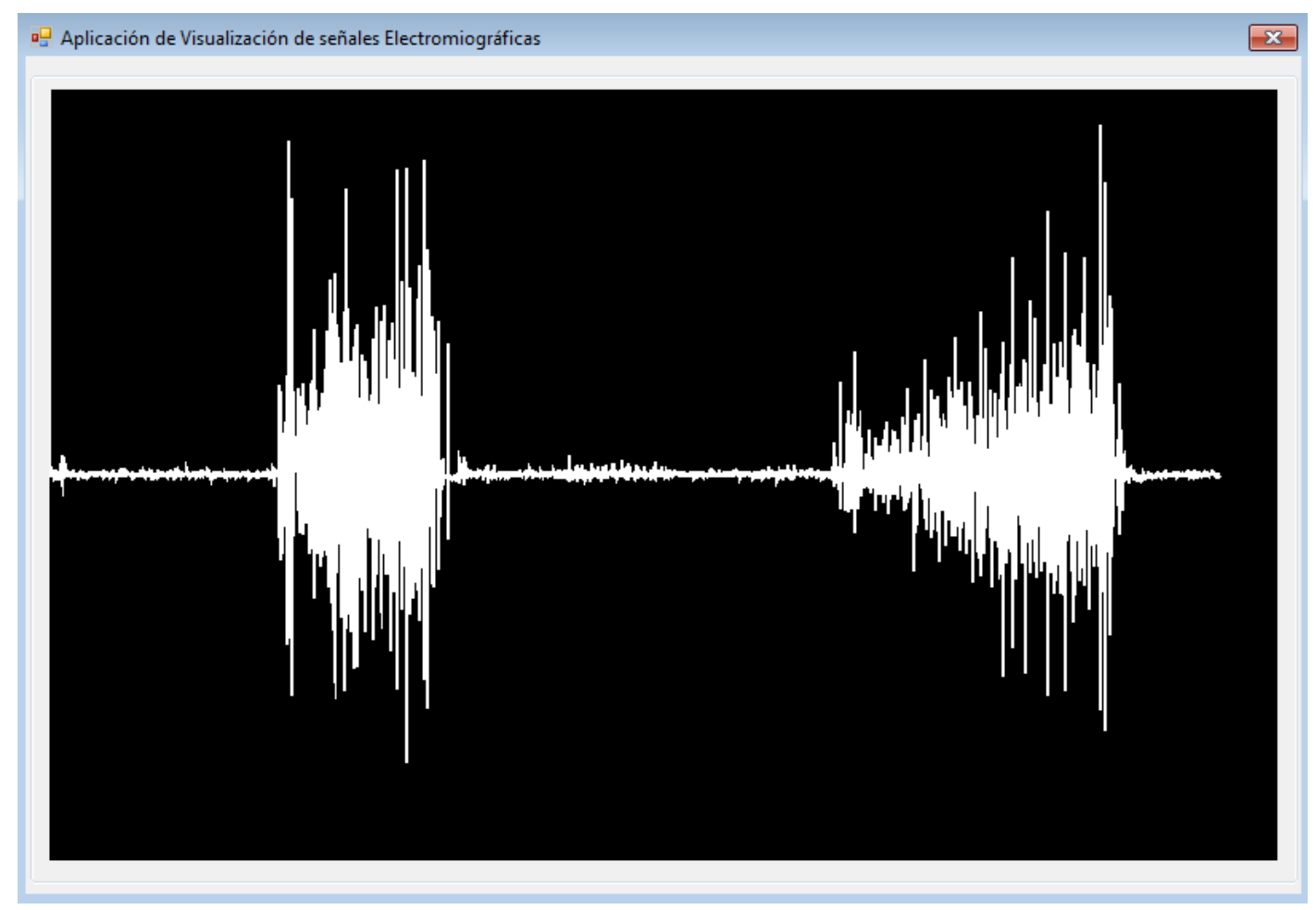

Figura 6-6: Captura del programa de visualización de la señal de EMG.

\subsubsection{Bloque para Simulink de Matlab}

Otro desarrollo de importancia para la investigación en el procesamiento de señales de EMG fue el desarrollo de un bloque representativo del dispositivo como adquisidor de datos en el entorno gráfico Simulink de Matlab. Esto permite testear y modificar rápidamente, en tiempo real, diferentes esquemas de procesamiento resultando muy versátil para la investigación en algoritmos de detección y su prueba en tiempo real (ver Figura 6-7). 


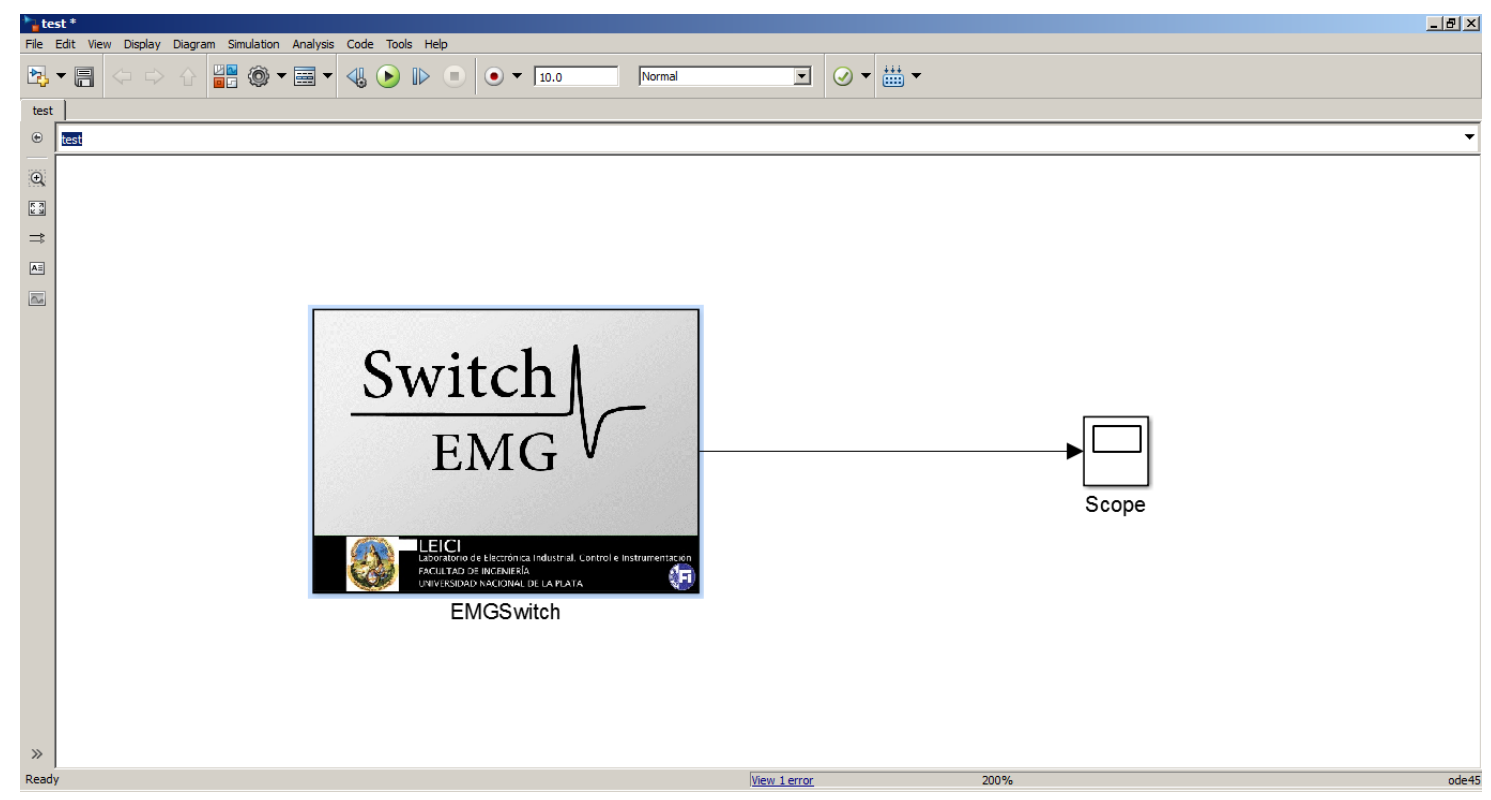

Figura 6-7: Captura de una simulación de Simulink utilizando el bloque para acceder a las señales del dispositivo.

\subsection{Ejemplos de aplicación del DA desarrollado}

\subsubsection{Teclado de Barrido de Windows}

El sistema operativo Microsoft Windows en sus últimas versiones, entre sus herramientas de accesibilidad, incorpora un teclado en pantalla que emula un teclado real, enviando las teclas seleccionada a la aplicación activa. Este tiene un modo de barrido automático, que permite controlarlo con el click del mouse, sin requerir el posicionamiento del cursor sobre las teclas. En este modo las teclas se van iluminando por grupos (Figura 6-8(a)), al hacer click (al contrer el usuario el músculo) el grupo que estaba iluminado es seleccionado y subdividido en subgrupos más pequeños de teclas (Figura 6-8(b)). El barrido ahora se realiza sobre estos subgrupos y al repetir el click quedará seleccionado el que estaba iluminado en dicho momento, comenzándose un último barrido tecla por tecla dentro de dicho subgrupo (Figura 6-8(c)).

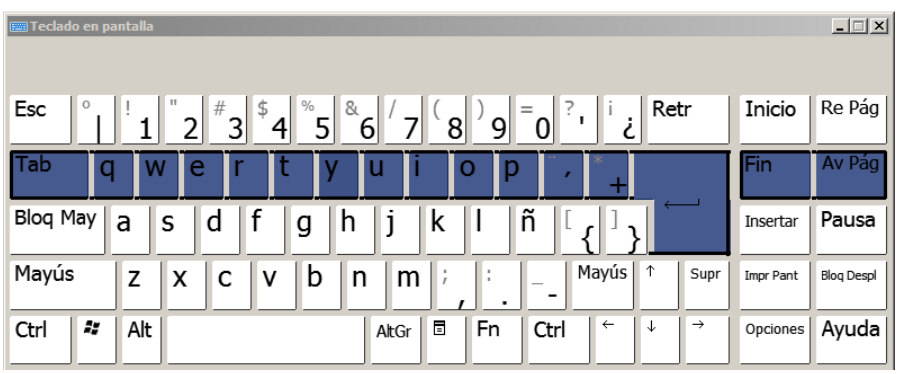



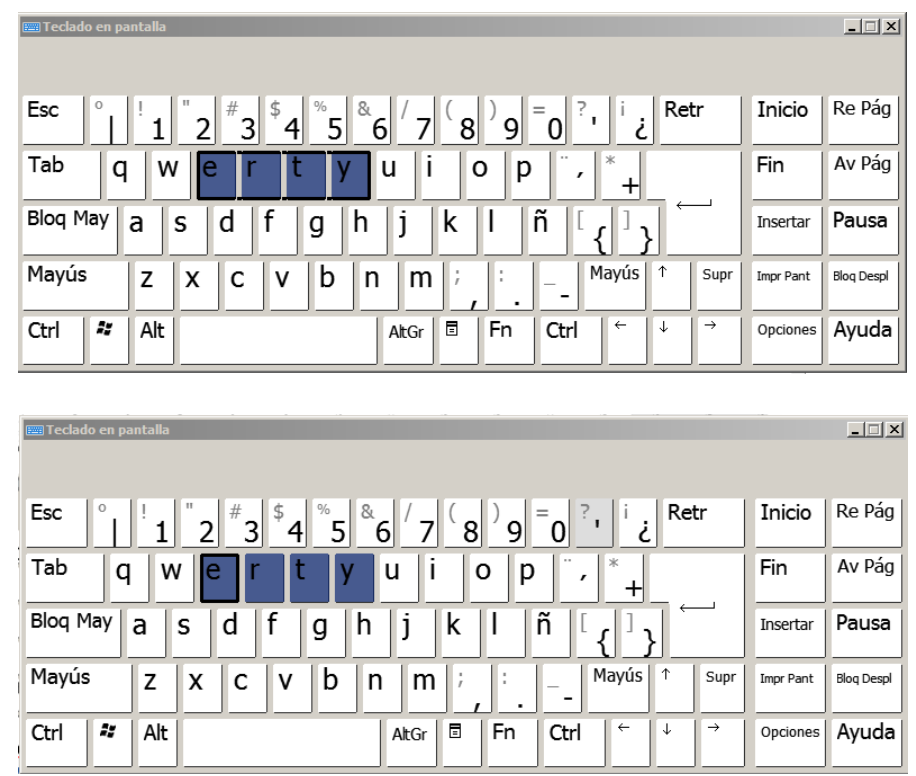

(b)

(c)

Figura 6-8: Secuencia de barrido del teclado en pantalla de Windows.

\subsubsection{Plaphoons}

Esta aplicación desarrollada por el Proyecto Fressa, de distribución gratuita, cuya función más básica es la comunicación por medio de imágenes. Sin embargo el mismo es muy flexible en su configuración y permite convertirlo en un teclado de barrido, con síntesis de voz y predicción de palabras. El mismo fue utilizado por la voluntaria con ELA, con una configuración de dos pantallas, en la primera (ver Figura 6-9(a)) se ubican las frases más frecuentes (que son leídas por un sintetizador de voz), un botón de alarma que dispara un sonido para llamar la atención del cuidador y un botón con el que pasa a la segunda pantalla, un teclado de barrido (ver Figura 6-9(b)) que posee predictor y permite escribir mensajes en un recuadro inferior, a la vez que permite la lectura por parte de un sintetizador y guardar el mensaje en un archivo. Al estar dispuestas las opciones en forma de grilla, la secuencia de barrido es por filas y una vez seleccionada la fila se barre opción por opción de la misma.

\begin{tabular}{|l|l|}
\hline ENFERMERO & Sonido \\
\hline \hline SI & NO \\
\hline \hline FRASE3 & FRASE4 \\
\hline \hline FRASE5 & FRASE6 \\
\hline TECLADO & \\
\hline
\end{tabular}

(a) 


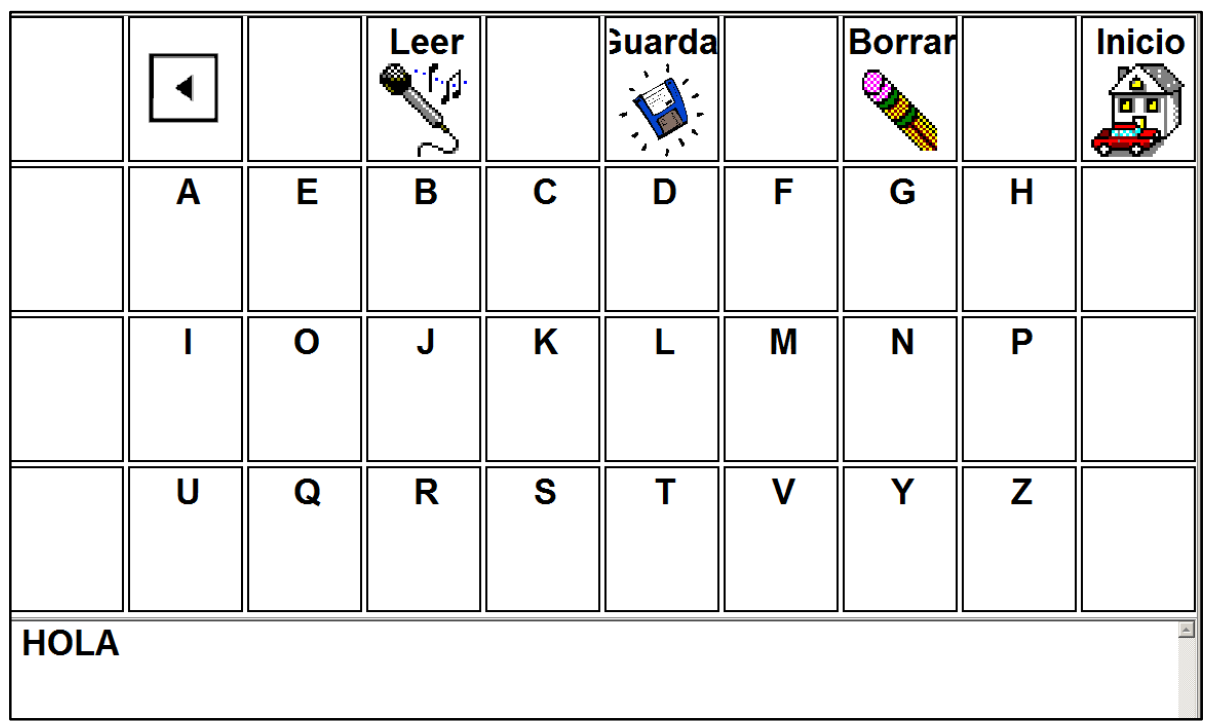

(b)

Figura 6-9: Pantallas del Plaphoons configuradas para la voluntaria con ELA.

\subsection{Conclusiones}

Se logró implementar un DA práctico, cumpliendo con los requisitos de que el mismo sea simple de operar y colocar, robusto, seguro y versátil. En dicha implementación se volcó el conocimiento adquirido a lo largo de la tesis, tanto en la instrumentación de biopotenciales y en el procesamiento de EMG como en las condiciones de operación real de esta clase de dispositivos.

La implementación utiliza componentes estándar y de costo relativamente bajo. Constituye una solución cerrada que puede ser transferida a la industria para su producción.

El hecho de que la salida esté basada en un click y sea vista por una PC como un mouse ordinario facilita su utilización con aplicaciones existentes así como con aplicaciones desarrolladas específicamente para las necesidades particulares de un usuario, dado que el desarrollador puede abstraerse de los detalles del DA y considerarlo un mouse (limitado a la realización de clicks). El dispositivo no solo es compatible con cualquier sistema operativo de PC sino con cualquier equipo USB host que pueda reconocer un mouse. 
Capítulo 6 - Implementación de un DA tipo switch 


\section{Capítulo 7}

\section{Conclusiones}

\subsection{Objetivos de la tesis}

El objetivo general de esta tesis fue, desde el principio, el estudio de diferentes técnicas de procesamiento de señales (tanto analógicas como digitales) y otros aspectos aplicables a los Dispositivos de Ayuda controlados por biopotenciales para personas con discapacidades motoras. Se planteó desde un principio la necesidad de implementar prototipos de DAs sobre los cuales poder evaluar su funcionamiento en tiempo real.

La dinámica propia del proceso de la tesis hizo que a medida que se fuese profundizando en el estudio de los distintos temas, surgiesen nuevos interrogantes y problemas que reorientaron la investigación y permitieron generar aportes originales a diferentes problemáticas relacionadas a los temas estudiados. Esto también contribuyó a replantear algunos de los objetivos originales y la tesis se fue orientando hacia un objetivo particular: la obtención de un DA controlado por EMG, del tipo switch, que sea útil para los usuarios, robusto, fácil de manipular por el usuario y por profesionales, seguro y versátil. En este dispositivo se integrarían varios de los aportes realizados a lo largo del trabajo de tesis.

\subsection{Conclusiones y Resultados de la tesis}

La tesis puede dividirse en dos partes. En la primera se focalizó en temas referidos a la instrumentación de biopotenciales y se aportaron soluciones para la adquisición robusta de biopotenciales frente a distintos escenarios de acoplamiento de EMI. La segunda parte, se orientó a resolver el objetivo particular de esta tesis de obtener un Dispositivo de Ayuda controlado por EMG del tipo switch, proponiéndose estrategias robustas para la detección de contracciones y soluciones prácticas de implementación.

A continuación se enumeran las conclusiones y resultados más relevantes de la tesis.

Obtención de parámetros del modelo EMI para distintas condiciones de utilización de Dispositivos de Ayuda controlados por Biopotenciales 
Los DA controlados por biopotenciales deben operar en ambientes domésticos bajo condiciones de interferencia electromagnéticas particulares que se alejan de los "valores típicos" que suelen ocurrir en ambientes controlados de laboratorio. La estimación experimental de los parámetros que definen el modelo de interferencia y sus valores para distintas condiciones de trabajo, descriptas en el Capítulo 2, permitieron estimar el impacto de la EMI en las medidas de biopotenciales y el diseño de técnicas para su reducción. Esto constituye un aporte importante, dado que estos parámetros se utilizaron en el Capítulo 4 para el diseño de los lazos de realimentación de modo común teniendo en cuenta su estabilidad.

\section{Evaluación y selección de distintos tipos de electrodos activos para EMG}

Los electrodos son un componente fundamental en la adquisición de biopotenciales, y una mala elección de los mismos puede perjudicar irreversiblemente el registro de las señales biológicas. En el Capítulo 3 se observó en registros de EMG, que luego de unos minutos, electros secos de acero inoxidable y electrodos húmedos de $\mathrm{Ag} / \mathrm{AgCl}$ muestran prácticamente el mismo desempeño, si se utilizan electrodos activos para transformar impedancia. La elección de uno sobre otro es una relación de compromiso entre la facilidad de colocación de los electrodos húmedos descartables y el mejor comportamiento de los electrodos secos en períodos de uso prolongado.

\section{Evaluación de Electrodos Capacitivos para la adquisición de EMG}

En cuanto a la utilización de electrodos capacitivos, se concluye que es una técnica viable para ser considerada en DAs controladas por EMG. La versatilidad lograda con este tipo de electrodos para registrar señales a través de materiales dieléctricos como prendas de vestir los hace ideales para dispositivos "vestibles" (wearable devices). Además, esta tecnología hace posible el desarrollo de equipos completamente aislados del usuario, es decir sin contacto galvánico, lo cual incrementa las condiciones de seguridad, facilitando también la comercialización y aceptación por parte de los usuarios. El punto débil de este tipo de electrodos son los mayores niveles de ruido, que demandan capacidades de acoplamiento más altas, implicando mayor área de electrodo. Esto último degrada la selectividad en las señales de EMG y hace a los electrodos capacitivos más voluminosos que sus contrapartes secos y húmedos.

\section{Reducción de interferencia de modo común mediante realimentación sintonizada. Implementación digital (DDRL) de la técnica propuesta y su utilización en sistemas de EEG multicanal Single-Ended}

Incorporando una función de transferencia sintonizada a la frecuencia de red $(50 \mathrm{~Hz})$, es posible mejorar la reducción de la tensión de modo común a esta frecuencia respecto de las soluciones clásicas. El nuevo circuito DRL digital, presentado en el Capítulo 4, reduce en más de $70 \mathrm{~dB}$ la interferencia de modo común de $50 \mathrm{~Hz}$. Este aporte original posibilita la implementación de sistemas de instrumentación con CMRR intrínsecamente bajo como arreglos de canales de EEG single-ended o electrodos preamplificados.

\section{Diseño e implementación de DRL Capacitivo (CDRL). Adquisición completamente aislada de ECG y EMG}

Los parámetros del modelo de EMI obtenidos en el Capítulo 2 permitieron el diseño optimizado del circuito DRL para el caso particular de electrodos capacitivos, descripto en el Capítulo 4. El prototipo implementado fue probado con sistemas capacitivos para la adquisición completamente aislada de ECG y EMG lográndose la captura de señales de muy buena calidad. Se concluye que sería viable la adquisición de EMG con sistemas completamente capacitivos, para la implementación de DAs.

\section{Sensor de EMG con amplificación diferencial y reducción de modo común in situ}

La proximidad entre los electrodos para el caso de señales de EMG, facilita el uso de electrodos activos con preamplificación porque pueden compartir circuitos auxiliares entre ellos. Incorporando un tercer electrodo es 
posible integrar un circuito DRL en el mismo arreglo, con lo cual se consigue un front-end compacto y con una muy alta inmunidad a las fuentes de interferencia. El sensor de EMG presentado en los Capítulos 3 y 4 se utilizó en el DA controlado por señales de EMG que se describe en el Capítulo 6 , que mostró ser muy robusto y confiable.

\section{Técnicas de procesamiento digital de EMG para la detección robusta de contracciones}

Las técnicas propuestas en el Capítulo 5 para la detección de contracciones musculares, evitan prácticamente cualquier ajuste sobre los parámetros de procesamiento, calculando el umbral a partir de las condiciones del nivel base. Las técnicas fueron ensayadas (y ajustadas) sobre registros reales de EMG de tres músculos distintos. $\mathrm{Si}$ bien no constituye una muestra significativa ya que todos los registros corresponden a la misma persona, los resultados son promisorios y muestran la aptitud de las estrategias propuestas para ser implementadas en un DA real. La prueba sobre la señal de la persona con ELA muestra que es posible aplicar los algoritmos sin recalibrar los parámetros de los mismos. El principal aspecto a resaltar es la robustez de las técnicas presentadas ante cambios del nivel base y su independencia de las características de la señal durante la contracción, lo que daría lugar a poder implementar interfaces que no requieran tediosas y repetitivas sesiones de ajuste, lo cual es muy ventajoso ya que se reducen los costos de mantenimiento y la capacitación de las personas que asisten al usuario del DA. Otro aspecto beneficioso de los algoritmos propuestos es la simplicidad de los mismos, lo cual permite implementarlos en microcontroladores de bajo costo, así como en dispositivos de bajo consumo.

\section{Desarrollo e implementación de prototipo de un Dispositivo de Ayuda controlado por EMG, de tipo switch}

Se logró implementar un DA práctico, cumpliendo con los objetivos de que el mismo sea simple de operar y colocar, robusto, seguro y versátil. Este es el resultado más importante de la tesis dado que integra varios de los resultados mencionados con anterioridad, volcando el conocimiento adquirido a lo largo de la tesis, tanto en la instrumentación de biopotenciales y en el procesamiento de EMG como en las condiciones de operación real de esta clase de dispositivos.

La implementación del DA utiliza componentes estándar y de costo relativamente bajo. Constituye una solución cerrada que puede ser transferida a la industria para su producción. El hecho de que la salida esté basada en un click y el equipo sea tratado por una PC como un mouse ordinario facilita su utilización con aplicaciones existentes así como con aplicaciones desarrolladas específicamente para las necesidades particulares de un usuario, dado que el desarrollador puede abstraerse de los detalles del DA y considerarlo un mouse (limitado a la realización de clicks). El dispositivo no solo es compatible con cualquier sistema operativo de PC sino con cualquier equipo USB host que pueda reconocer un mouse, por ejemplo una tablet o celular.

\subsection{Líneas de trabajo en el futuro}

A continuación se mencionan algunas líneas de trabajo que tienen continuidad con la presente tesis y que se desean abordar en el futuro.

\section{Utilización de electrodos capacitivos y CDRL en Dispositivo de Ayuda controlado por EMG, de tipo switch}

A partir de los resultados mencionados se concluye que es viable la adquisición completamente aislada (sin contacto galvánico) de señales de EMG superficial, es decir utilizando en conjunto electrodos capacitivos y el CDRL. La utilización de esta tecnología en un DA implementa intrínsecamente una barrera de aislación entre el usuario y el resto de los potenciales, a la vez que facilita el desarrollo de DAs vestibles, ya que sería posible capturar los biopotenciales a través de prendas de vestir.

Uno de los aspectos que obstaculizan la incorporación de esta tecnología en Dispositivos de Ayuda es el mayor tamaño de los electrodos respecto de sus equivalentes húmedos/secos para mantener similares niveles de ruido, 
lo cual no solo implica electrodos más voluminosos (y posiblemente menos confortables) sino también menos selectividad en la captura de las señales de EMG.

\section{Medición sin contacto de la tensión de línea}

Un problema técnicamente similar al de la instrumentación aislada de biopotenciales con electrodos capacitivos, pero en una aplicación completamente diferente y que despierta creciente interés es la medición de tensión de línea sin contacto. La adaptación de las técnicas conocidas para biopotenciales permitiría medir la tensión de red en cualquier punto de una instalación eléctrica a través de la vaina aislante, sin tener que acceder a un terminal de conexión o retirar la vaina en algún punto. Si bien el problema es similar a la medición de biopotenciales, las soluciones presentadas en la tesis no pueden aplicarse directamente dado que se presentan algunas particularidades a resolver como por ejemplo que la entrada del seguidor de tensión estará a la tensión de línea, requiriendo alguna solución particular para su alimentación y protección.

\section{Medición sin contacto de impedancia biológica y su extensión a la tomografía de impedancia}

La medición de impedancia en distintas zonas del cuerpo es una herramienta habitual en aplicaciones biomédicas. A partir de los valores absolutos o de sus variaciones, es posible tener conocimiento de distintos fenómenos fisiológicos, como por ejemplo los ritmos cardíaco y respiratorio, o la grasa corporal. Generalmente se requieren cuatro electrodos superficiales: dos para la inyección de corriente y dos para la medición de tensión. Esta técnica, simple en su principio, es el elemento constitutivo de la Tomografía de Impedancia Eléctrica (EIT: Electrical Impedance Tomography), que a partir de un número de medidas independientes de impedancia, tomadas entre distintos electrodos que rodean un área del cuerpo, reconstruye una imagen que muestra los cambios de conductividad que se dan internamente en los distintos tejidos. En general estas mediciones de impedancia se realizan colocando electrodos metálicos sobre la piel, en algunos casos con el agregado de electrolitos líquidos o en gel, inyectando una corriente por dos de ellos y midiendo tensión en los electrodos restantes. Se desea estudiar la utilización de la tecnología de electrodos capacitivos a estas técnicas, pero en este caso, no solo debe considerarse la medición de tensión de manera aislada, sino también la inyección de corriente.

\subsection{Publicaciones}

\section{Publicaciones realizadas en el marco de esta tesis}

- M. Haberman, E. Spinelli, P. García y F. Guerrero, "Capacitive Driven-Right-Leg Circuit Design", International Journal of Biomedical Engineering and Technology, ISSN 1752-6418, Vol. 17, no. 2, pp. 115-126, 2015.

- M. Haberman y E. Spinelli "Dispositivos de asistencia a discapacitados motores: Switch controlado por señales electromiográficas" Segundas Jornadas de Investigación y Transferencia 2013. Secretaría de Investigación y Transferencia, Facultad de Ingeniería, UNLP. ISBN 978-950-34-09466. Mayo 2013.

- M. Haberman y E. Spinelli, "A multichannel EEG acquisition scheme based on single ended amplifiers and Digital DRL", IEEE Transactions on Biomedical Circuits and Systems, ISSN 1932-4545, Issue 6, Vol. 6, diciembre 2012.

- M. Haberman y E. Spinelli, "Diseño de $\mathrm{HCl}$ controlada por señales electromiográficas faciales", XIV Reunión de Trabajo en Procesamiento de la Información y Control (RPIC 2011), noviembre de 2011. 
- M. Haberman y E. Spinelli, "Metodología de diseño de una Interfaz Hombre-Computador controlada por señales electromiográficas faciales para persona con Esclerosis Lateral Amiotrófica", XVIII Congreso Argentino de Bioingeniería (SABI 2011), setiembre de 2011.

- M. Haberman, A. Cassino y E. Spinelli, "Estimation of stray coupling capacitances in biopotential measurements", Medical and Biological Engineering and Computing, ISSN 0140-0118, Issue 9, Vol. 49, pp. 1067-1071. 2011.

- M. Haberman y E. Spinelli, "A Digital Driven Right Leg Circuit," 32nd Annual International Conference of the IEEE Engineering in Medicine and Biology Society, Buenos Aires, Argentina. ISBN 978-142444124-2, 2010.

- E. Spinelli y M. Haberman, "Insulating electrodes: a review on biopotential front-ends for dielectric skin-electrode interfaces," Physiological Measurement, Special Issue on Electrode-Skin linterface, ISSN 0967-3334. 2010.

\section{Otras publicaciones relacionadas con la tesis}

- E. Spinelli, F. Guerrero, P. García y M. Haberman, "A simple and reproducible capacitive electrode". Medical Engineering \& Physics. 2016. Vol. 38, No. 3, pp. 286-289, Marzo de 2016.

- F. N. Guerrero; E. M. Spinelli; M. A. Haberman, "Analysis and Simple Circuit Design of Double Differential EMG Active Electrode," IEEE Transactions on Biomedical Circuits and Systems , ISSN 1932-4545, En prensa, diciembre de 2015.

- F. Guerrero, M. Haberman y E. Spinelli, "Sistema Multicanal Para Adquisición de Biopotenciales", Revista Ingeniería Biomédica, ISSN 1909-9762, Vol. 8, № 15, pp. 18-26, Enero-Junio de 2014.

- E. Spinelli, M. Haberman, P. García y F. Guerrero "Electrodos Capacitivos. Captura de señales biomédicas sin contacto" Segundas Jornadas de Investigación y Transferencia 2013. Secretaría de Investigación y Transferencia, Facultad de Ingeniería, UNLP. ISBN 978-950-34-0946-6. Mayo 2013.

- F. Guerrero, E. Spinelli y M. Haberman, "Dispositivo Multicanal Para Adquisición de Biopotenciales" Segundas Jornadas de Investigación y Transferencia 2013. Secretaría de Investigación y Transferencia, Facultad de Ingeniería, UNLP. ISBN 978-950-34-0946-6. Mayo 2013.

- E. Spinelli y M. Haberman, "Noise Analysis of Fully Differential Circuits" Revista IEEE América Latina, ISSN 1548-0992, Vol. 10, No. 4, pp. 1889-1892, 2012.

- E. Spinelli, G. Hornero, O. Casas y M. Haberman, "A Design Method for Active High-CMRR FullyDifferential Circuits," International Journal of Instrumentation Technology. ISSN 2043-7854, 2012, vol. 1, no 2, p. 103-113.

- E. Spinelli, M. Haberman, P. García y F. Guerrero, "A capacitive electrode with fast recovery feature," Physiological Measurement, ISSN 0967-3334, Julio 2012.

- P. García, E. Spinelli, G. Toccaceli y M. Haberman, "Interface Cerebro Computador basada en potenciales evocados visuales de estado estacionario: ensayos preliminares", XVIII Congreso Argentino de Bioingeniería (SABI 2011), setiembre de 2011.

- M. Haberman, G. Toccaceli, E. Spinelli y P. García, "Arquitectura de Software de Tiempo Real para Interfaz Cerebro-Computadora,"Anales del XXII Congreso Argentino de Control Automático AADECA 2010. Ciudad de Buenos Aires, 2010. 
- P. García, M. Haberman y E. Spinelli, "A Versatile Hardware Platform for Brain Computer Interfaces," 32nd Annual International Conference of the IEEE Engineering in Medicine and Biology Society, Buenos Aires, Argentina. ISBN 978-1-42444124-2, 2010.

- M. Haberman, P. García y E. Spinelli: "Comparación de dos amplificadores de EEG para Interfaces Cerebro-Computadora: JAICC 2009". II Jornadas Argentinas sobre Interfaces Cerebro-Computadora JAllCC 2009, Paraná, Argentina, abril de 2009.

- P. García, E. Spinelli y M. Haberman: "Plataformas de Hardware para interfaces cerebro computadora: JAICC 2009". II Jornadas Argentinas sobre Interfaces Cerebro-Computadora JAIICC 2009, Paraná, Argentina, abril de 2009.

- E. Spinelli, P. García y M. Haberman: "Paradigmas ICC para validación de plataformas autónomas". II Jornadas Argentinas sobre Interfaces Cerebro-Computadora JAIICC 2009, Paraná, Argentina, abril de 2009.

- M. Haberman. "Adquisición de biopotenciales en disposiciones multicanales". XXI Congreso Argentino de Control Automático AADECA. Buenos Aires, 2008. 


\section{Referencias}

Aleksandrowicz, A y Leonhardt, S. (2007) Wireless and Non-contact ECG Measurement System the Aachen SmartChair, Acta Polytechnica Vol. 47 No. 4-5, pp. 68-71

Amatniek, E. (1958). Measurement of Bioelectric Potentials With Microelectrodes and Neutralized Input Capacity Amplifiers. IRE Transactions on Medical Electronics, PGME-10, pp. 3-14.

Andreasen, D. (2005). EMG Switch Activation Algorithms and Methods EMG Single Switch Activation Algorithms and Methods. Online: http://emgswitch.com/publications/

Aso, S., Sasaki, A., Hashimoto, H. y Ishii, C. (2006). Driving Electric Car by Using EMG Interface. 2006 IEEE Conference on Cybernetics and Intelligent Systems.

Axelson, J. (2005) USB complete: everything you need to develop custom USB peripherals. Lakeview re- search.

Baek, H, Chung, G, Kim, K and Park, K. (2012). A Smart Health Monitoring Chair for Nonintrusive Measurement of Biological Signals', IEEE Transactions on Information Technology in Biomedicine, Vol. 16, pp. 150-158

Barreto, A., Scargle, S. y Adjouadi, M. (2000). A practical EMG-based human-computer interface for users with motor disabilities. Journal of Rehabilitation Research and Development, 37(1), pp. 5363.

Bates, R. (2002). A Computer Input Device Selection Methodology for Users with High-Level Spinal Cord Injuries. In Proceedings of the 1st Cambridge Workshop on Universal Access and Assistive Technology (CWUAAT), pp. 1-4.

Caamaño, M. A., Bonell, C. E., Cherniz, A. S., \& Tabernig, C. B. (2011). Muscular Contraction Onset Detection from Surface Electromyogram Signal to the Command of Functional Electrical Stimulators, IEEE Latin America Transactions, vol. 9, no. 1, pp. 779-783.

CanAssist (Online). http://www.canassist.ca/EN/main/programs/technologies-and-devices/ communication/headband-operated-emg-switch.html 
Chi, Y, Ng, P, Kang, E, Kang, J, Fang, J y Cauwenberghs, G. (2010b). Wireless non-contact cardiac and neural monitoring. Wireless Health 2010, pp. 15-23

Chi, Y. M., Jung, T., \& Cauwenberghs, G. (2010a). Dry-Contact and Noncontact Biopotential Electrodes: Methodological Review. IEEE Reviews in Biomedical Engineering, 3, pp. 106-119.

Clancy, E., y Hogan, N. (1997). Relating agonist-antagonist electromyograms to joint torque during isometric, quasi-isotonic, nonfatiguing contractions. IEEE Transactions on Bio-Medical Engineering, 44(10), pp. 1024-8.

Clark, J. (2009). The origin of biopotentials. In J. G. Webster (Ed.),Medical instrumentation: application and design. John Wiley \& Sons, pp. 126-188

Controlbionics (Online). http://www.controlbionics.com/

Davies, T. C., Mudge, S., Ameratunga, S., y Stott, N. S. (2010). Enabling self-directed computer use for individuals with cerebral palsy: a systematic review of assistive devices and technologies. Developmental Medicine and Child Neurology, 52(6), pp. 510-6

De Luca, C. J. (1997), The use of surface electromyography in biomechanics. Journal of Applied Biomechanics, 13 (2): pp. 135-163.

De Luca, C. J. (2006). Electromyography. In J. G. Webster (Ed.), Encyclopedia of Medical Devices and Instrumentation John Wiley \& Sons, pp. 98-109.

Degen, T., \& Jäckel, H. (2004). Enhancing interference rejection of preamplified electrodes by automated gain adaption. IEEE Transactions on Bio-Medical Engineering, 51(11), pp. 2031-9.

Deutsch, S., \& Deutsch, A. (1993). Understanding the nervous system: an engineering perspective. New York: IEEE Press.

Di Fabio, R. P. (1987). Reliability of computerized surface electromyography for determining the onset of muscle activity. Physical Therapy, 67(1), pp. 43-8.

Eilebrecht, B, Schommartz, A, Walter, M, Wartzek, T, Czaplik, M y Leonhardt, S. (2010), = A capacitive ECG array with visual patient feedback', Proceedings of the 32th Annual International Conference of the IEEE Engineering in Medicine and Biology Society, pp. 6539-6542

Farina, D., Merletti, R., y Stegeman, D. F. (2004). Biophysics of the generation of EMG signals. Electromyography: physiology, engineering, and noninvasive applications, pp. 81-105.

Fischer, R., Groensfelder, T., \& Nordmann, R. (2005). Alternative control system for operating a PC using intentional muscle contractions only. Online-Proceedings of the 2005 CSUN, pp. 1-6.

Fridlund, A. J., y Cacioppo, J. T. (1986). Guidelines for Human Electromyographic Research. Psychophysiology, 23(5), pp. 567-589.

García, P., Spinelli, E., Toccaceli, G., y Haberman, M. (2011). Interfaz Cerebro Computador basada en potenciales evocados visuales de estado estacionario: ensayos preliminares. XVIII Congreso Argentino de Bioingeniería SABI 2011. 
Gazetalk (Online) http://wiki.cogain.org/index.php/Gazetalk

Geddes, L., y Valentinuzzi, M. E. (1973). Temporal changes in electrode impedance while recording the electrocardiogram with "Dry" electrodes. Annals of Biomedical Engineering, 1(3), pp. 356367.

Graimann, B., Allison, B., y Pfurtscheller, G. (2010). Brain-computer interfaces: A gentle introduction. Brain-Computer Interfaces, pp. 1-28.

Guerrero, F. N., Haberman, M., y Spinelli, E. (2014). Sistema multicanal para adquisición de biopotenciales. Revista Ingeniería Biomédica, 8(15), pp. 18-26.

Haberman, M. A., y Spinelli, E. M. (2012). A Multichannel EEG Acquisition Scheme Based on Single Ended Amplifiers and Digital DRL. IEEE Transactions on Biomedical Circuits and Systems, 6(6).

Haberman, M., Cassino, A., y Spinelli, E. (2011). Estimation of stray coupling capacitances in biopotential measurements. Medical \& Biological Engineering \& Computing, 49(9), pp. 1067-71.

Haberman, M., García, P. y Spinelli, M. (2009) Comparación de dos amplificadores de EEG para Interfaces Cerebro-Computadora: JAICC 2009. II Jornadas Argentinas sobre Interfaces CerebroComputadora JAllCC 2009, Paraná, Argentina.

Hansen, J. P., Lund, H., Aoki, H., y Itoh, K. (2006). Gaze communication systems for people with ALS. In Proc. of the Int. Symp. on ALS/MND'06, pp. 35-38.

Henderson, S., Skelton, H., y Rosenbaum, P. (2008). Assistive devices for children with functional impairments: impact on child and caregiver function. Developmental Medicine and Child Neurology, 50(2), pp. 89-98.

Hodges, P. W., y Bui, B. H. (1996). A comparison of computer-based methods for the determination of onset of muscle contraction using electromyography. Electroencephalography and Clinical Neurophysiology/Electromyography and Motor Control, 101(6), pp. 511-519.

Huhta, J. C., y Webster, J. G. (1973). 60-HZ interference in electrocardiography. IEEE transactions on bio-medical engineering, 20(2), pp. 91-101.

Huigen, E., Peper, A., y Grimbergen, C. (2002). Investigation into the origin of the noise of surface electrodes. Medical and Biological Engineering ,(March),pp. 332-338.

IEC-60601-1 (2005) Medical Electrical Equipment - Part 1: General requirements for basic safety and essential performance, 3ed. IEC subcommittee 62A: Common aspects of electrical equipment used in medical practice..

Jiang, M., Rahmani, A., Westerlund, T., Liljeberg, P., y Tenhunen, H. (2015). Facial Expression Recognition with sEMG Method. In Proc. of IEEE International Conference on Computer and Information Technology (CIT)

Kim, K, Lim, Y y Park, K. (2005). Common mode noise cancellation for electrically non-contact ECG measurement system on a chair. Conference Proceedings: Annual International Conference of the IEEE Engineering in Medicine and Biology Society. 6, pp. 5881-3 
Leeb, R., y Sagha, H. (2011). A hybrid brain-computer interface based on the fusion of electroencephalographic and electromyographic activities. Journal of neural Engineering, 8, pp.1-5

Levkov, C. (1988). Amplification of biosignals by body potential driving. Analysis of the circuit performance. Medical and Biological Engineering and Computing.

Lim, Y, Chung, G y Park, K. (2010) Capacitive driven-right-leg grounding in Indirect-contact ECG measurement, Proceedings of the 32th Annual International Conference of the IEEE Engineering in Medicine and Biology Society, pp.1250-53

López, N. M. (2010). Procesamiento de Señales Electromiográficas Superficiales para el Control de Dispositivos Robóticos.

Martinsen, O., y Grimnes, S. (2011). Bioimpedance and bioelectricity basics.

Mason, S., Kronegg, J., Huggins, J., Fatourechi, M., y Schlögl, A. (2006). Evaluating the Performance of Self-Paced Brain-Computer Interface Technology. Tech Report, 0, pp. 1-60. Retrieved from http://ipl.ece.ubc.ca/bci_files/self_paced_tech_report-2006-05-19.pdf

Matthews, R., McDonald, N. J., Fridman, I., Hervieux, P., y Nielsen, T. (2005). The invisible electrode - zero prep time, ultra low capacitive sensing. In Proceedings of the 11th International Conference on Human-Computer Interaction, pp. 22-27.

McFarland, D., Miner, L., Vaughan, T., y Wolpaw, J. (2000). Mu and beta rhythm topographies during motor imagery and actual movements. Brain topography.

Merletti, R., Botter, A., Troiano, A., Merlo, E., y Minetto, M. A. (2009). Technology and instrumentation for detection and conditioning of the surface electromyographic signal: state of the art. Clinical biomechanics (Bristol, Avon), 24(2), pp. 122-34.

Merletti, R., y Parker, P. (2004). Electromyography: physiology, engineering, and non-invasive applications.

Merlo, A., Farina, D., y Merletti, R. (2003). A fast and reliable technique for muscle activity detection from surface EMG signals. IEEE Transactions on Biomedical Engineering, 50(3), pp. 316-323.

Metting Van Rijn, A. C., Peper, A., y Grimbergen, C. (1990). High-quality recording of biolectric events. Part 1 Interference reduction, theory and practice. Medical \& Biological Engineering \& Computing, 28, pp. 389-397

Moon, I., Lee, M., Chu, J., y Mun, M. (2005). Wearable EMG-based HCl for electric-powered wheelchair users with motor disabilities. In Proceedings - IEEE International Conference on Robotics and Automation (ICRA 2005) Barcelona, España. pp. 2649-2654.

Moritani, T., Stegeman, D., y Merletti, R. (2004). Basic physiology and biophysics of EMG signal generation. Electromyography Physiology Engineering and Noninvasive Applications.

Müller-Putz, G. R., Breitwieser, C., Cincotti, F., Leeb, R., Schreuder, M., Leotta, F y Millán, J. D. R. (2011). Tools for Brain-Computer Interaction: A General Concept for a Hybrid BCl. Frontiers in Neuroinformatics, 5(November), 30. 
Neuman, M. R. (2009a). Biopotential electrodes. Medical instrumentation: application and design. John Wiley \& Sons, pp. 189-240.

Neuman, M. R. (2009b). Biopotential amplifiers. Medical instrumentation: application and design. John Wiley \& Sons, pp. 241-292.

Niemenlehto, P. (2009). Detection of Physiological Events from Biomedical Signals Originating from Facial Landmarks.

Nilas, P., Rani, P. y Sarkar N. (2004). An innovative high-level human-robot interaction for disabled persons. IEEE International Conference on Robotics and Automation, 2004, 3, pp. 2309-2314.

Pallàs-Areny, R., y Colominas, J. (1991). Simple, fast method for patient body capacitance and power-line electric interference measurement. Medical \& Biological Engineering \& Computing, 29(5), pp. 561-563

Pallás-Areny, R., y Webster, J. G. (1999).Analog signal processing. John Wiley \& Sons.

Park, H., Kwon, S., Kim, H., y Park, K. (1999). ADAPTIVE EMG-DRIVEN COMMUNICATION FOR THE DISABILITY, Proceedings of The First Joint BMES/EMBS Conference 41, pp. 7803.

Pfurtscheller, G., y Neuper, C. (1997). Motor imagery activates primary sensorimotor area in humans, Neuroscience Letters, 239, pp. 65-68.

Pfurtscheller, G., y Neuper, C. (2002). Dynamics of Sensorimotor Oscillations in a Motor Task. Braincomputer interfaces.

Plonsey, R., y Barr, R. C. (2007). Bioelectricity, a quantitative approach. ISBN: 9780387488646

Porat, B. (1997) A Course in Digital Signal Processing, John Wiley and Sons, New York.

Prance, R. J., Debray, A., Clark, T. D., Prance, H., Nock, M., Harland, C. J., y Clippingdale, A. J. (2000). An ultra-low-noise electrical-potential probe for human-body scanning. Measurement Science and Technology, 11(3), pp. 291-297

Proyecto Fressa (Online). http://projectefressa.blogspot.com.ar/

Rabiner, L. R. y Gold, B. (1975), Theory and application of digital signal processing, Prentice-Hall, Englewood Cliffs, N.J..

Rani, P., y Sarkar, M. S. (2005). EMG-based high level human-robot interaction system for people with disability. ROMAN 2005. IEEE International Workshop on Robot and Human Interactive Communication, 2005., pp. 280-285.

Richardson, P.C. (1967) The insulated electrode: a pasteless electrocardiographic technique Proc. Annu. Conf. on Engineering in Medicine and Biology vol 7, pp. 9-15

Schlögl, A., Kronegg, J., Huggins, J. E., y Mason, S. G. (2007). Evaluation Criteria for BCI Research. Toward Brain-Computer Interfacing.

Searle, A., y Kirkup, L. (2000). A direct comparison of wet, dry and insulating bioelectric recording electrodes. Physiological Measurement,21(2), pp. 271. 
SENIAM, Surface Electromyography for Noninvasive Assessment of Muscle.(Online). http://www.seniam.org

Sierra Araujo, B. (2006). Aprendizaje Automático: conceptos básicos y avanzados. Aspectos prácticos utilizando el software Weka. ISBN 10-84-8322.

Spinelli, E. y Mayosky, M., (2000) Interfaz Adaptiva para comunicaciones mediante EEG basadas en el Ritmo Alfa Visual, XVIII Congreso de la Sociedad Española de Ingeniería Biomédica, Cartagena España,, pp. 161-163.

Spinelli, E.(2007). Amplificadores de Instrumentación en Aplicaciones Biomédicas

Spinelli, E., y Haberman, M. (2010). Insulating electrodes: a review on biopotential front ends for dielectric skin-electrode interfaces. Physiological Measurement, 31(10), pp. 183-98.

Spinelli, E.; Martinez, N.H.; Mayosky, M. (1999), A transconductance driven-right-leg circuit, in IEEE Transactions on Biomedical Engineering, vol.46, no.12, pp.1466-1470

Staude, G., \& Wolf, W. (1999). Objective motor response onset detection in surface myoelectric signals. Medical Engineering and Physics, 21(6-7), pp. 449-467.

Staude, G., Flachenecker, C., Daumer, M., y Wolf, W. (2001). Onset detection in surface electromyographic signals: A systematic comparison of methods. Eurasip Journal on Applied Signal Processing, 2001(2), pp. 67-81.

Steffen, M, Aleksandrowicz, A y Leonhardt, S. (2007), Mobile Noncontact Monitoring of Heart and Lung Activity, IEEE Transactions on Biomedical Circuits and Systems, Vol. 1, pp. 250-257

Stone, J. V. (2004) Independent Component Analysis: A Tutorial Introduction, Cambridge, MA : MIT Press , ISBN 0-262-69315-1

Sullivan, T. J., Deiss, S. R., y Cauwenberghs, G. (2007). A Low-Noise, Non-Contact EEG/ECG Sensor. In 2007 IEEE Biomedical Circuits and Systems Conference, pp. 154-157

Tassinary, L. G., Cacioppo, J. T., y Green, T. R. (1989). A psychometric study of surface electrode placements for facial electromyographic recording: I. The brow and cheek muscle regions. Psychophysiology, 26(1), pp. 1-16.

Tsui, C. S. L., Jia, P., Gan, J. Q., Hu, H., y Yuan, K. (2007). EMG-based Hands-Free Wheelchair Control with EOG Attention Shift Detection. In Proceedings of the 2007 IEEE International Conference on Robotics and Biometrics. pp. 1266-1271

Ueno, A., Uchikawa, Y., y Noshiro, M. (2007). A capacitive sensor system for measuring Laplacian electromyogram through cloth: a pilot study. Conference Proceedings: Annual International Conference of the IEEE Engineering in Medicine and Biology Society, pp. 5732-5734

Walter, M., Eilebrecht, B., Wartzek, T., y Leonhardt, S. (2011). The smart car seat: personalized monitoring of vital signs in automotive applications. Personal and Ubiquitous Computing, 15(7), pp. 707-715.

Webster, J. (2009). Medical instrumentation: application and design. John Wiley \& Sons. 
Williams, M. R., y Kirsch, R. F. (2008). Evaluation of head orientation and neck muscle EMG signals as command inputs to a human-computer interface for individuals with high tetraplegia. IEEE Transactions on Neural Systems and Rehabilitation Engineering, 16(5), pp. 485-96

Winter, B. B., y Webster, J. G. (1983a). Driven-right-leg circuit design. IEEE Transactions on BioMedical Engineering, 30(1), pp. 62-6.

Winter, B. B., y Webster, J. G. (1983b). Reduction of interference due to common mode voltage in biopotential amplifiers. IEEE transactions on bio-medical engineering, 30(1), pp. 58-62.

Wolpaw, J. y Boulay, C. (2002) Brain Signals for Brain-Computer Interfaces. Brain-computer interfaces.

Wolpaw, J., Birbaumer, N., McFarland, D. J., Pfurtscheller, G., y Vaughan, T. M. (2002b). Braincomputer interfaces for communication and control. Clinical neurophysiology : official journal of the International Federation of Clinical Neurophysiology, 113(6), pp. 767-91. 